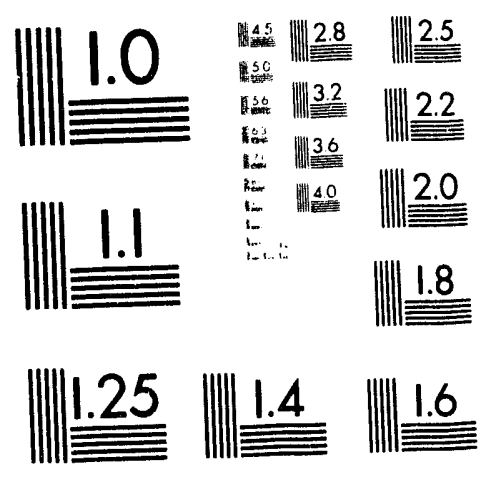



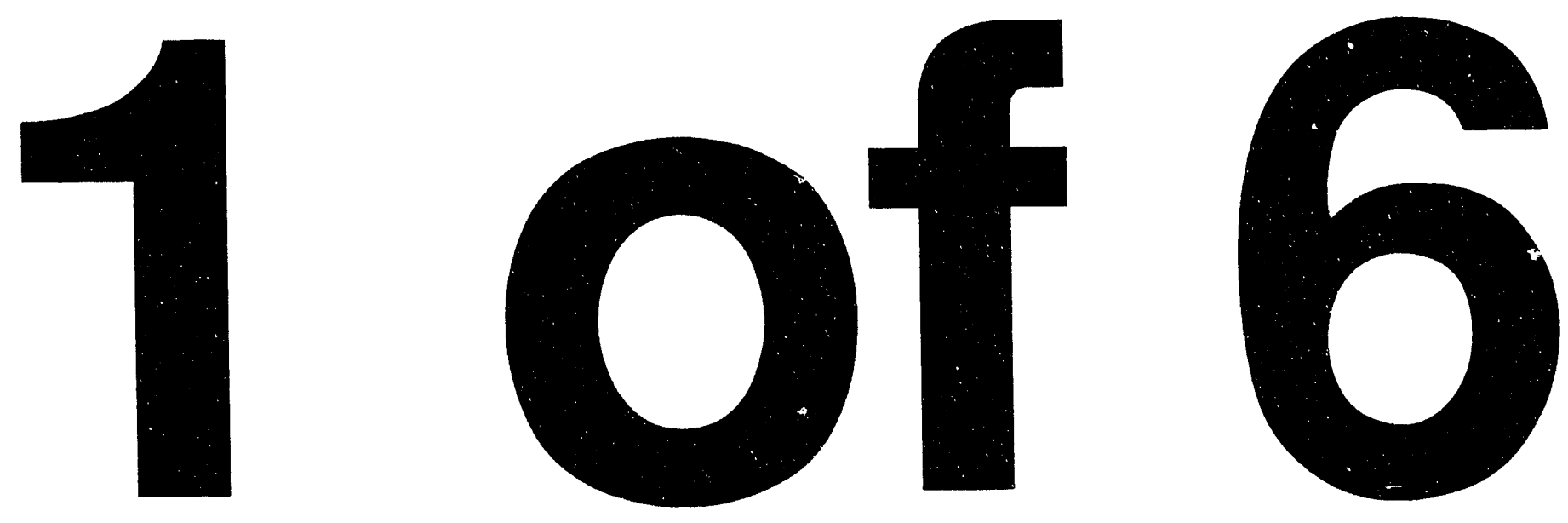
WHC-EP-0625

UC. 721

\title{
Hanford Site Waste Storage Tank Information Notebook
}

\author{
E.I. Husa \\ R. E. Raymond \\ R. K. Welty \\ S. M. Griffith \\ B. M. Hanlon \\ R. R. Rios \\ N. J. Vermeulen
}

Date Published

July 1993

Prepared for the U.S. Department of Energy Office of Environmental Restoration and Waste Management

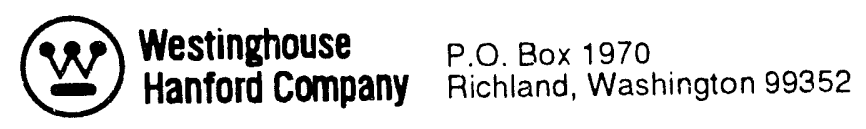

Hanford Operations and Engineering Contractor for the

US Department of Energy under Contract DE-AC06.87RL10930

Approved for Public Release 


\section{LEGAL DISCLAIMER}

This report was prepared as an account of work sponsored by an agency of the United States Government. Neither the Unired States Government nor any agency inereof, nor any of their employees, nor any of their contractors. subcontractors or their employees, makes any warranty, exoress or implied, or assumes any legal liability or responsibility for the accuracy, complgteness, or any third party's use or the results of such use of any information, apparalus, product, or process disclosed, or represents that its use would not infringe privately owned rights. Reference herein to any specific commercial product, process, es service by trade name. Irademark, manufacturer, or otherwise, does not necessarily conslitute or imoly its endorsement. recommendalion, or lavoring by the United Siales Government or any agency thereof or its contraciors or subcontraciors. The views and opinions of authors expressec herein do not necessarily state or reflect those of the United Siales Government or any agency thereof

This report has been reproduced from the best available copy. Avallable in paper copy and microficne.

Available to the U.S. Department of Energy and is contractors from

Office of Scientific and Technical Information P. O. Box 62

Oak Ridge, TN 37831

(615) 576.8401

Avaitable to the public from the U.S. Department of Commerce National Technical Information Service

5285 Port Royal Road

Springfield. VA 22161

(703) 487.4650

Printed in the Unitod Stalos of Amorica 
WHC-EP-0625

Document Title:

Prepared by:

Approved by:
Hanford Site Waste Storage Tank Information Notebook

Elver Aura

E. I. Hush,

Engineer

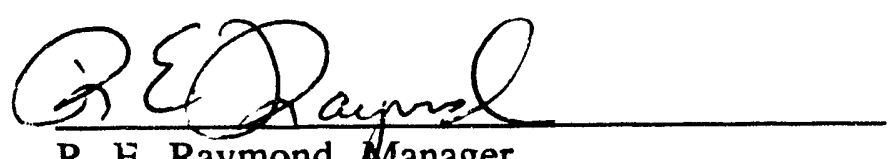

R. E. Raymond, Manager

Single-Shell Tank Technology
$13 \sqrt{1} 4993$

Date

$\frac{14}{\text { Date }}$ felly 93

iii 


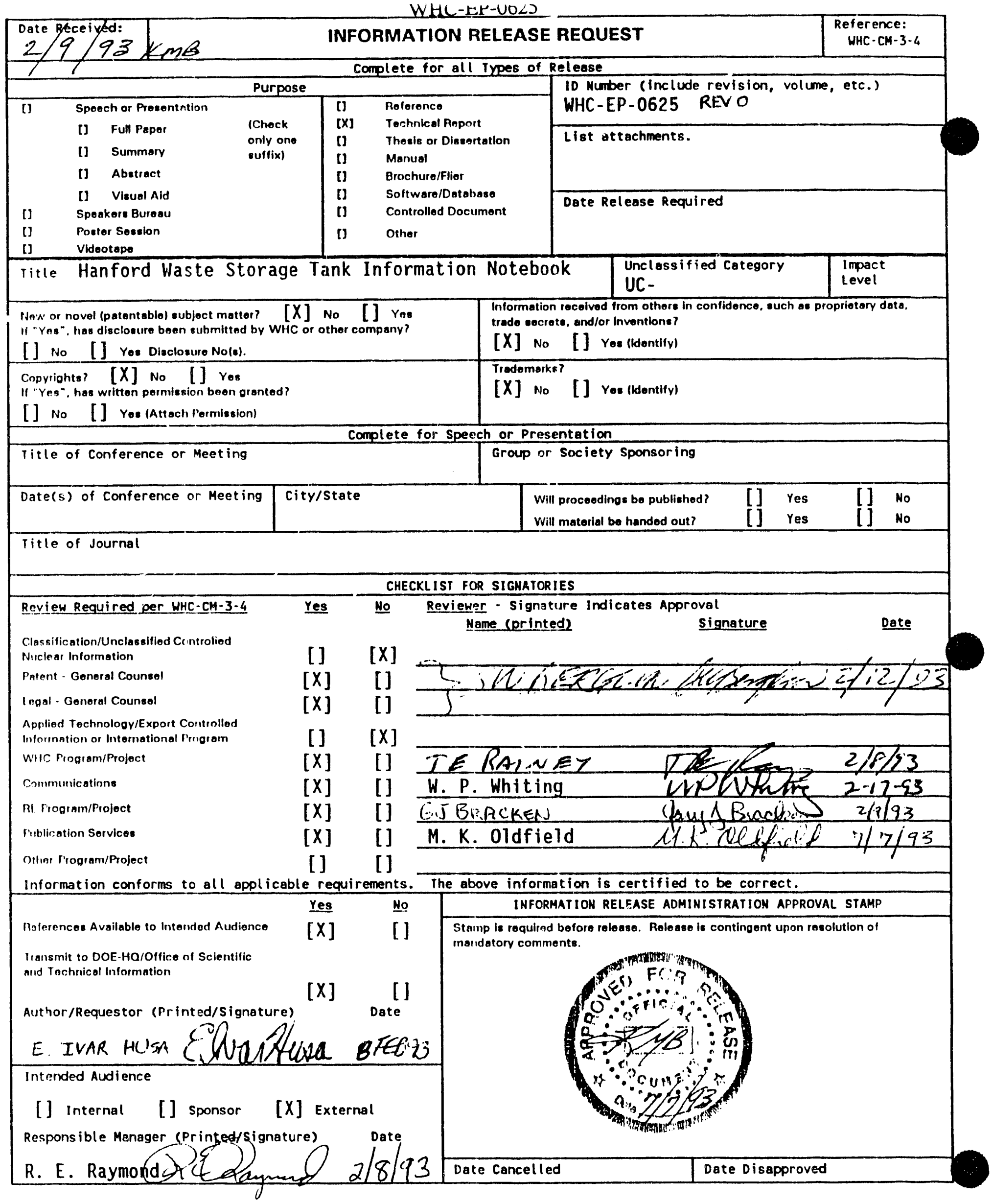


WHC-EP-0625

\title{
HANFORD SITE WASTE STORAGE TANK INFORMATION NOTEBOOK
}

\begin{abstract}
This report provides summary data on the radioactive waste stored in underground tanks in the 200 East and West Areas at the Hanford Site. The summary data covers each of the existing 161 Series 100 underground waste storage tanks (500,000 gallons and larger). It also contains information on the design and construction of these tanks. The information in this report is derived from existing reports that document the status of the tanks and their materials. This report also contains interior, surface photographs of each of the 54 Watch List tanks, which are those tanks idenrified as Priority I Hanford Site Tank Farm Safety Issues in accordance with Public Law 101-510, Section 3137*.
\end{abstract}

* National Defense Authorizution Act for Fiscal Year 1991, Public Law 101-510, "Safety' Measures for Measures for Waste Tanks at Hanford Nuclear Reservation," Section 3137 (Wyden Amendment). 
WHC-EP-0625

This page intentionally left blank. 


\section{CONTENTS}

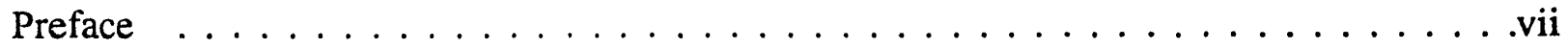

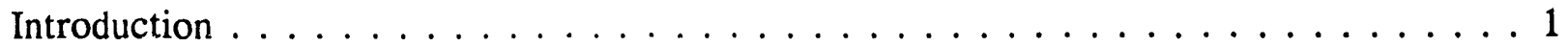

Summary Tank Data Sheets . . . . . . . . . . . . . . . . . . . . . 19

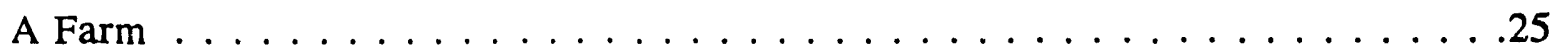

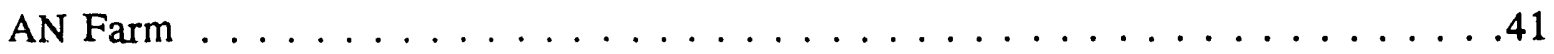

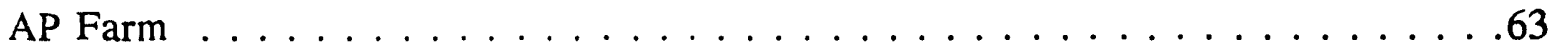

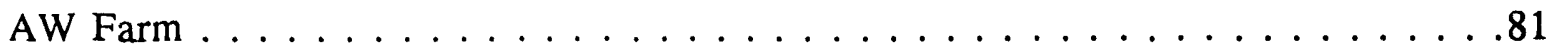

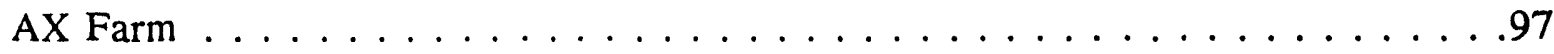

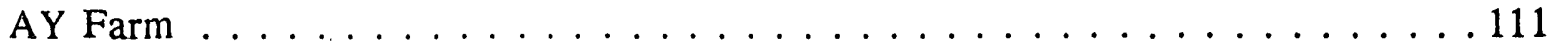

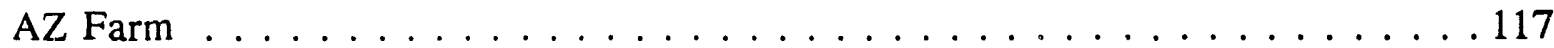

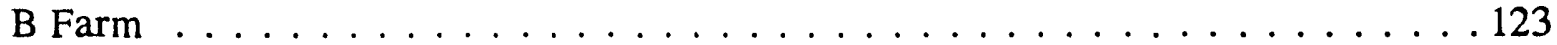

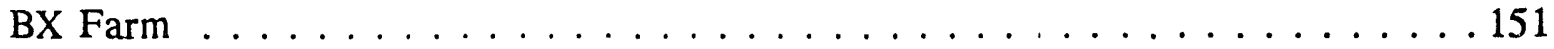

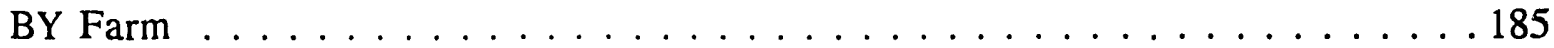

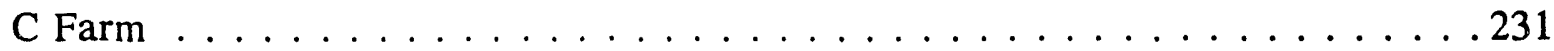

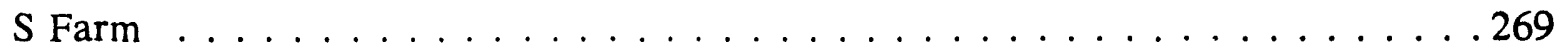

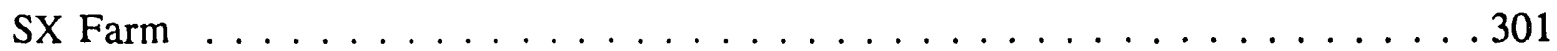

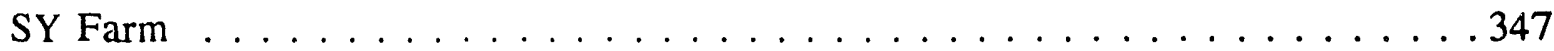

T Farm . . . . . . . . . . . . . . . . . . . . . . .359

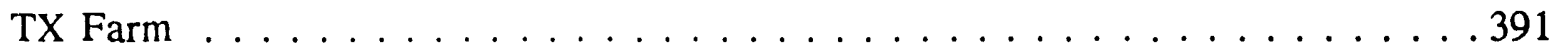

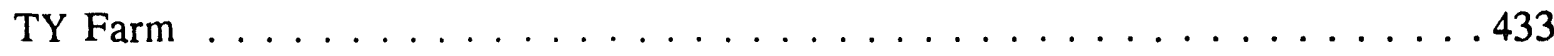

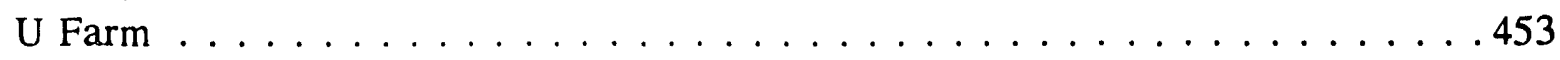

Glossary . . . . . . . . . . . . . . . . . . . . . . . . . . 4491

References .........................501 


\section{LIST OF FIGURES}

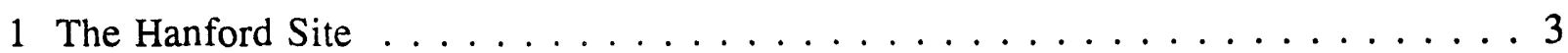

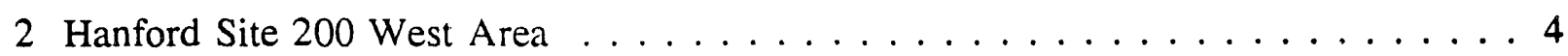

3 Hanford Site 200 East Area ...................... . . . .

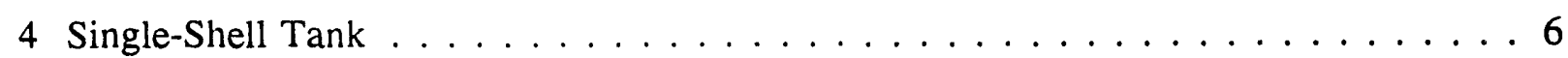

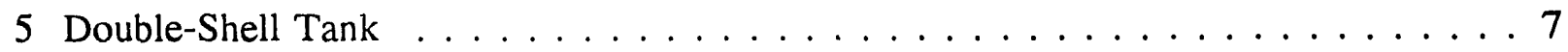

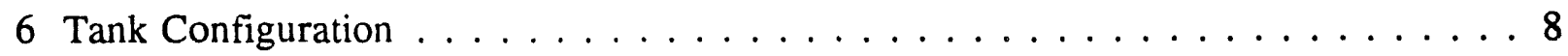

7 Hanford Tank Farm Facilities $\ldots \ldots \ldots \ldots \ldots \ldots$

8 Watch List Tank Quick Reference . . . . . . . . . . . . . . . 11

9200 West Single-Shell Tank Contents . . . . . . . . . . . . . . . . . . . 13

10200 East Single-Shell Tank Contents . . . . . . . . . . . . . . . 15

11 Double Shell Tank Contents . . . . . . . . . . . . . . . 17

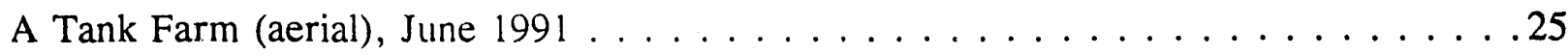

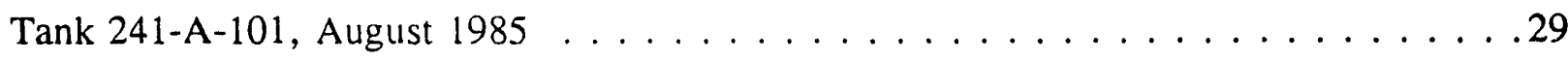

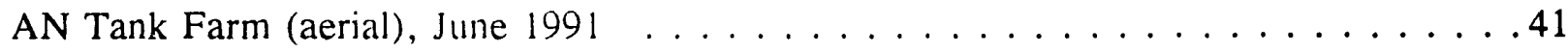

Tank 241-AN-103, October $1987 \ldots \ldots \ldots \ldots$

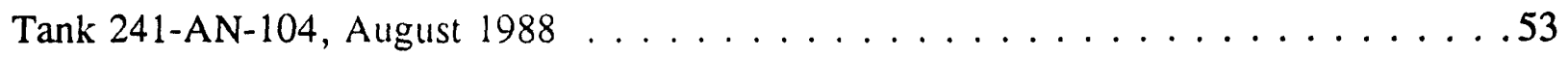

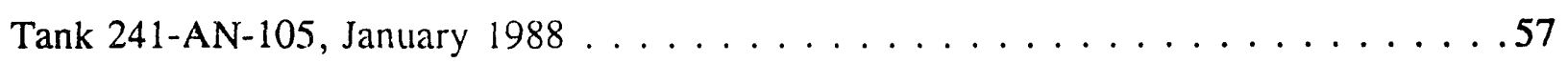

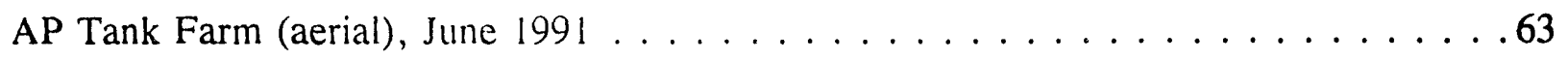

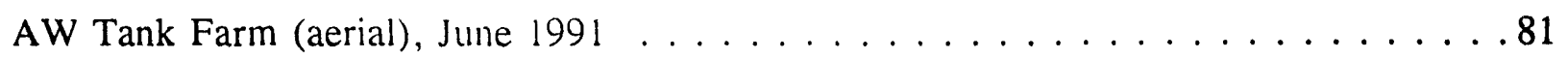

Tank 241-AW-101, March $1988 \ldots \ldots \ldots \ldots$

AX Tank Farm (aerial), June $1991 \ldots \ldots \ldots \ldots$. . . . . . . . . . . . . . . . . . 


\section{LIST OF FIGURES (continued)}

Tank $241-A X-101$, August $1987 \ldots \ldots \ldots \ldots 1$

Tank 241-AX-103, August $1987 \ldots \ldots \ldots$. . . . . . . . . . . . . . . . . . .

AY Tank Farm (aerial), June $1991 \ldots \ldots \ldots \ldots \ldots \ldots \ldots$

AZ Tank Farm (aerial), June $1991 \ldots \ldots \ldots \ldots \ldots \ldots$

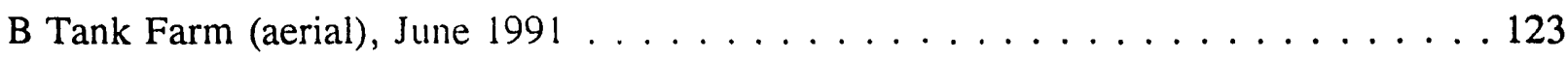

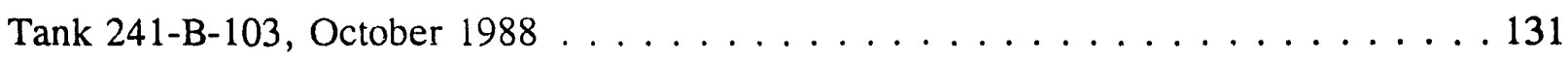

BX Tank Farm (aerial), June $1991 \ldots \ldots \ldots \ldots \ldots \ldots$

Tank 241-BX-102, November $1978 \ldots \ldots \ldots \ldots$

Tank 241-BX-106, May $1988 \ldots \ldots \ldots \ldots \ldots$

Tank $241-$ BX-110, July $1985 \ldots \ldots \ldots \ldots \ldots$

Tank $241-B X-111$, September $1985 \ldots \ldots \ldots \ldots 18 \ldots \ldots \ldots \ldots$

BY Tank Farm (aerial), June $1991 \ldots \ldots \ldots \ldots$

Tank 241-BY-101, September $1981 \ldots \ldots$. . . . . . . . . . . . 189

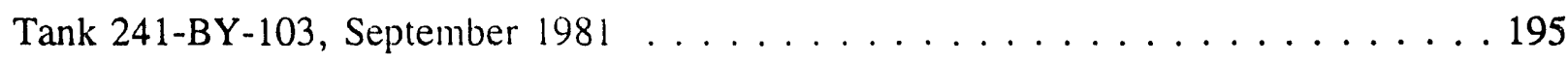

Tank $241-B Y-104$, February $1981 \ldots \ldots$. . . . . . . . . . . . . . 199

Tank $241-$ BY-105, Jily $1986 \ldots \ldots \ldots \ldots$

Tank 241-BY-106, November $1982 \ldots \ldots$. . . . . . . . . . . . 207

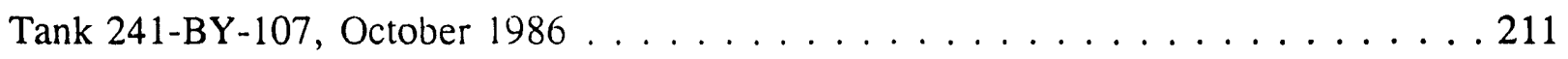

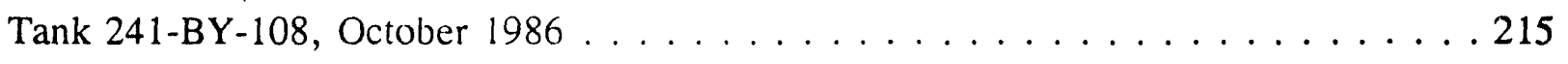

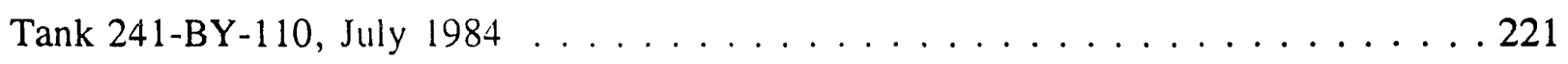

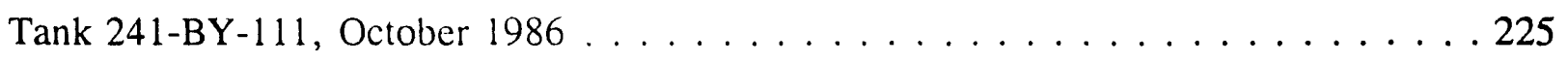




\section{LIST OF FIGURES (continued)}

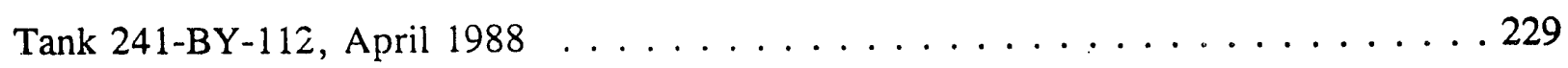

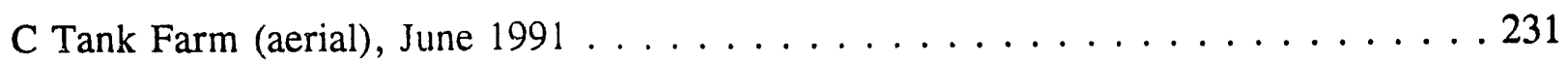

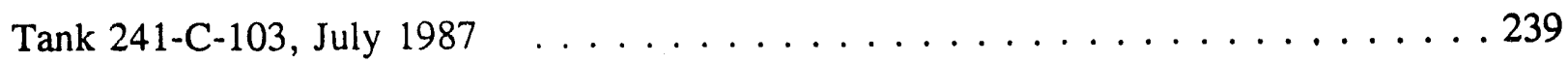

Tank 241-C-106, April $1979 \ldots \ldots \ldots \ldots$. . . . . . . . . . . . . . . . . . . .

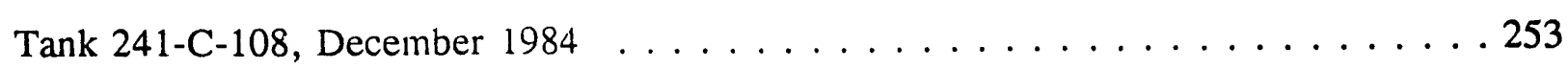

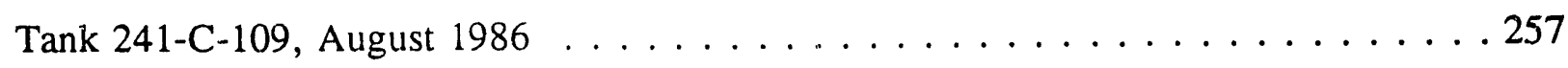

Tank $241-\mathrm{C}-111$, February $1970 \ldots \ldots \ldots \ldots$

Tank $241-C-112$, July $1990 \ldots \ldots$. . . . . . . . . . . . . . . 267

$\mathrm{S}$ Tank Farm (aerial), June $1991 \ldots \ldots . \ldots \ldots$

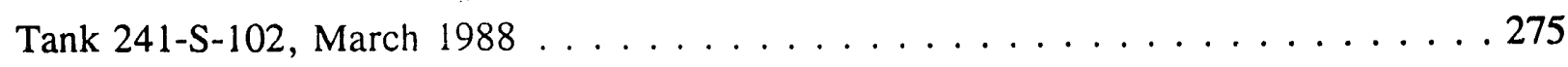

Tank 241-S-111, August $1989 \ldots \ldots \ldots$. . . . . . . . . . . . . . . . . . . . . . . . .

Tank 241-S-112, March $1987 \ldots \ldots$. . . . . . . . . . . . . . . . . 299

SX Tank Farm (aerial), June $1991 \ldots \ldots \ldots$. . . . . . . . . . . . . . . . . . .

Tank 241-SX-101, March $1989 \ldots \ldots \ldots$. . . . . . . . . . . . . . . . . . . . .

Tank 241-SX-102, January $1988 \ldots \ldots$. . . . . . . . . . . . . . . . . . . . . . . . .

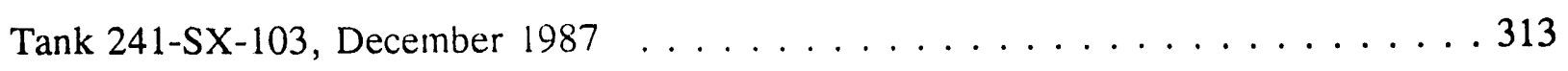

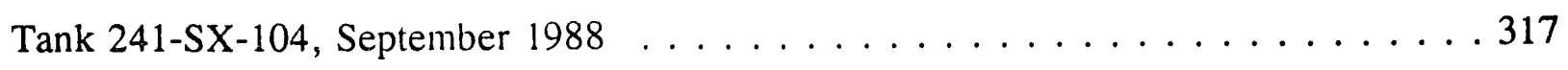

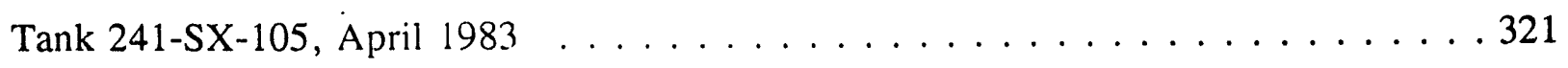

Tank $241-S X-106$, June $1989 \ldots \ldots$. . . . . . . . . . . . . . . . 325

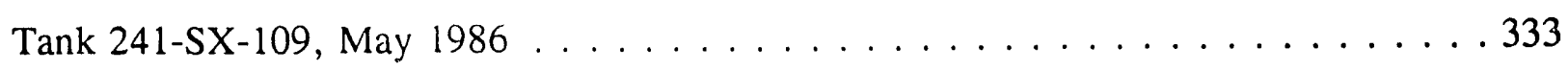

SY Tank Farm (aerial), June $1991 \ldots \ldots \ldots \ldots$. . . . . . . . . . . . . . . . . . . . 
WHC-EP-0625

\section{LIST OF FIGURES (continued)}

Tank 241-SY-101, April $1989 \ldots \ldots \ldots \ldots \ldots$. . . . . . . . . . . . . . . . .

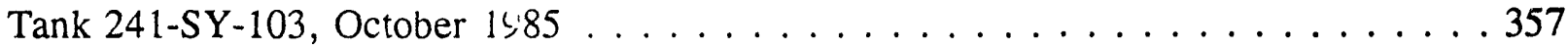

T Tank Farm (aerial), June $1991 \ldots \ldots \ldots \ldots \ldots \ldots$

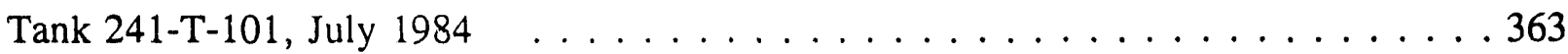

Tank 241-T-107, July $1984 \ldots \ldots$. . . . . . . . . . . . . . . 377

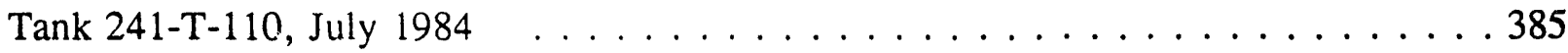

TX Tank Farm (aerial), June $1991 \ldots \ldots \ldots$. . . . . . . . . . . . 391

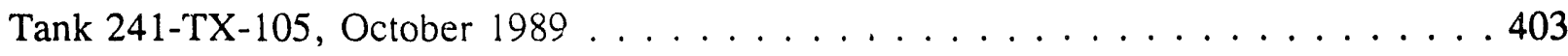

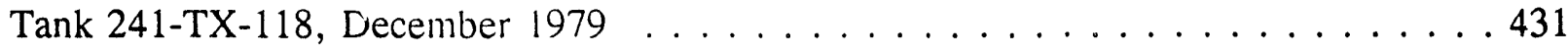

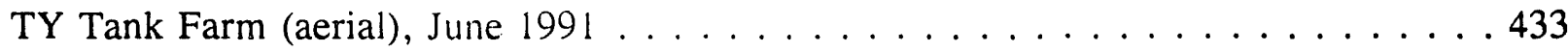

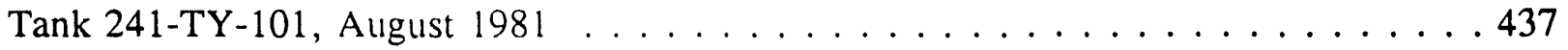

Tank 241-TY-103, August $1989 \ldots \ldots \ldots \ldots \ldots \ldots$. . . . . . . . . . . . . . . . . . . .

Tank 241-TY-104, November $1987 \ldots \ldots \ldots$. . . . . . . . . . . . 447

U Tank Farm (aerial), June $1991 \ldots \ldots \ldots \ldots \ldots \ldots$

Tank 241-U-103, September $1988 \ldots \ldots \ldots$. . . . . . . . . . . . . 461

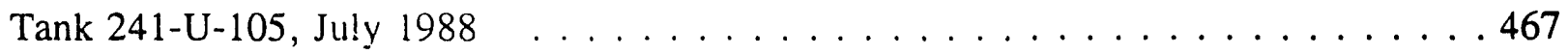

Tank 241-U-106, July $1988 \ldots \ldots \ldots \ldots \ldots \ldots$. . . . . . . . . . . . . . . . . .

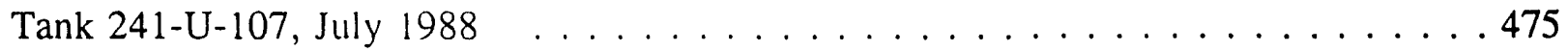

Tank 241-U-108, September $1984 \ldots \ldots$. . . . . . . . . . . . . . . . . . . . .

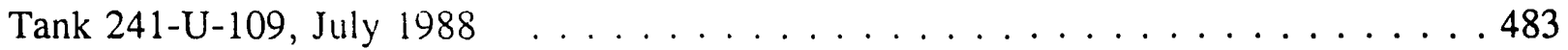


WHC-EP-0625

This page intentionally left blank. 


\section{PREFACE}

The Hanford Waste Storage Tank Information Notebook was a collaborative effort. The primary authors were E. Ivar Husa, Richard E. Raymond, and Richard K. Weliy of the Single-Shell Tank Technology group. Other contributors were S.M. Griffith, B.M. Hanlon, R.R. Rios, and N.J. Vermeulen of the Surveillance and Data Acquisition group. 


\section{LIST OF TERMS}

$\begin{array}{ll}\text { ASTM } & \text { American Society for Testing and Materials } \\ \text { CAM } & \text { continuous air monitoring } \\ \text { CASS } & \text { computer automated survillance system } \\ \text { CC } & \text { concentrated complexant } \\ \text { DC } & \text { dilute complexed waste } \\ \text { double-container reciver tank } \\ \text { DCRT } & \text { diaphragm-operated valve } \\ \text { DOV } & \text { double-shell tank } \\ \text { DST } & \text { double-shell slurry } \\ \text { DSS } & \text { ethylenediaminetetra-acetic acid } \\ \text { EDTA } & \text { Food Instrument Company } \\ \text { FIC } & \text { hydroxyethylenediaminetriacetic acid } \\ \text { HEDTA } & \text { high-efficiency particulate air } \\ \text { HEPA } & \text { iminodiacetate } \\ \text { IDA } & \text { interstitial liquid level } \\ \text { ILL } & \text { liquid level } \\ \text { LL } & \text { liguid observation well } \\ \text { LOW } & \text { neutralized cladding removal waste } \\ \text { NCAW } & \text { Plutonium Finishing Plant } \\ \text { PFP } & \text { Plutonium Uranium Reduction Extraction (Plant) } \\ \text { PUREX } & \text { Surveillance Analysis Computer System } \\ \text { SACS } & \text { Surveillance and Data Acquisition section } \\ \text { SDA } & \text { single-shell tank } \\ \text { SST } & \text { thermocouple } \\ \text { TC } & \text { transuranic waste } \\ \text { TRU } & \text { underground storage tank } \\ \text { UST } & \end{array}$




\section{INTRODUCTIÚN}

\section{OBJECTIVE}

This draft document consolidates summary data about the Hanford Site's underground waste tanks from several different sources. This document provides general, summary information and is not meant to replace the source documents, which contain detailed information on these tanks. Westinghouse Hanford Company (WHC) developed this report at the request of the U.S. Department of Energy, High-Level Waste Safety Division, Office of Hanford Programs.

\section{DESCRIPTION OF REPORT}

This notebook has three parts: introductory text, summary tank data sheets, and a glossary of tank terms. The introductory text provides a brief overview of the Hanford Site waste tanks, including figures that summarize the content and status of the tank farms. Summary data on each tank are located in the tank data sheet section. A detailed description of each data field is found at the beginning of that section. The glossary contains the definitions of many terms used in conjunction with the Hanford Site's waste tanks and also contains the list of source documents.

All 161 of the 100 Series waste tanks (75-foot internal diameter, with a nominal capacity of from 500,000 gallons up to $1,100,000$ gallons) are included in the summary tank data sheets in the second section of the report. No information is included on the smaller 200 Series tanks, all 16 of which are 55,000-gallon tanks. Future revisions of this report will include the 200 Series tanks.

Special information has been added for the 54 Watch List tanks. Watch List tanks are those tanks that have been identified as Priority 1 Hanford Site Tank Farm Safety Issues, in accordance with Public Law 101-510, Section 3137, "Safety Measures for Waste Tanks at Hanford Nuclear Reservation." Waste surface photographs are included only for the Watch List tanks.

Many details about construction, operation, stabilization, and current status are reported on all of the data sheets. Some data, such as waste volumes, surface levels, and temperatures change over time and this report is based on the most recent documented data available from the references and sources.

\section{TANK OVERVIEW}

The Hanford Site occupies 560 square miles along the Columbia River in Washington State (see Figure 1). The underground waste storage tanks are clustered in rectangular 
arrays of 2 to 18 tanks called tank farms; there are currently 18 tank farms on the Hanford Site. The tank farms are located in the 200 West and 200 East Areas near the center of the Hanford Site (see Figures 2 and 3 for the location of tanks within these areas). The facility prefix 241 is part of each farm and tank name, for example: 241-AN Tank Farm and tank 241-AN-101.

Between 1943 and 1964, 149 single-shell tanks (SSTs) were built on the Hanford Site. SSTs are arranged in 12 farms containing 4 to 18 tanks each. Wastes have not been added to any SST since November 1980 . However, water is currently added to 2 tanks for evaporative cooling purposes. SSTs are lined with American Society for Testing Materials (ASTM) A283 Grade C or A201 Grade C (only the 241-AX Tank Farm) carbon steel. The liners are encased by a reinforced concrete shell (see Figure 4).

The first double-shell tanks (DSTs), which went into service in May 1971, are arranged in farms of 2 to 8 tanks each. These tanks are comprised of a heat-treated (stressrelieved) primary carbon steel liner (shell) and a non-stress-relieved outer steel liner, both inside the reinforced concrete shell (see Figure 5). An annulus between the two shells traps any waste leaking from the primary shell.

Typical tank configurations for SSTs and DSTs are shown in Figure 6. The top of each tank is at least 6 feet below grade, providing radiological shielding. Tank access for monitoring is provided by risers penetrating the tank dome. Existing risers range from 4 to 42 inches in diameter. A variety of instruments monitor tank contents and the surrounding soil. Typical instrumentation configurations for single- and double-shell tanks are depicted in Figures 4 and 5, respectively.

\section{TANK STATUS SUMMARIES}

Figures 7 through 11 offer a quick overview of some aspects of tank farm status. Figure 7, Hanford Tank Farm Facilities, contains many details found in the tank data sheets. However, this figure shows the drywell locations throughout a farm, not just one tank at a time as shown in the tank data sheet section. Figure 8 highlights the distribution of the Watch List tanks across the site. Figures 9 and 10 graphically depict the tank contents for 200 West and 200 East Areas SSTs; Figure 11 does the same for the DSTs. 
Figure 1. The Hanford Site.

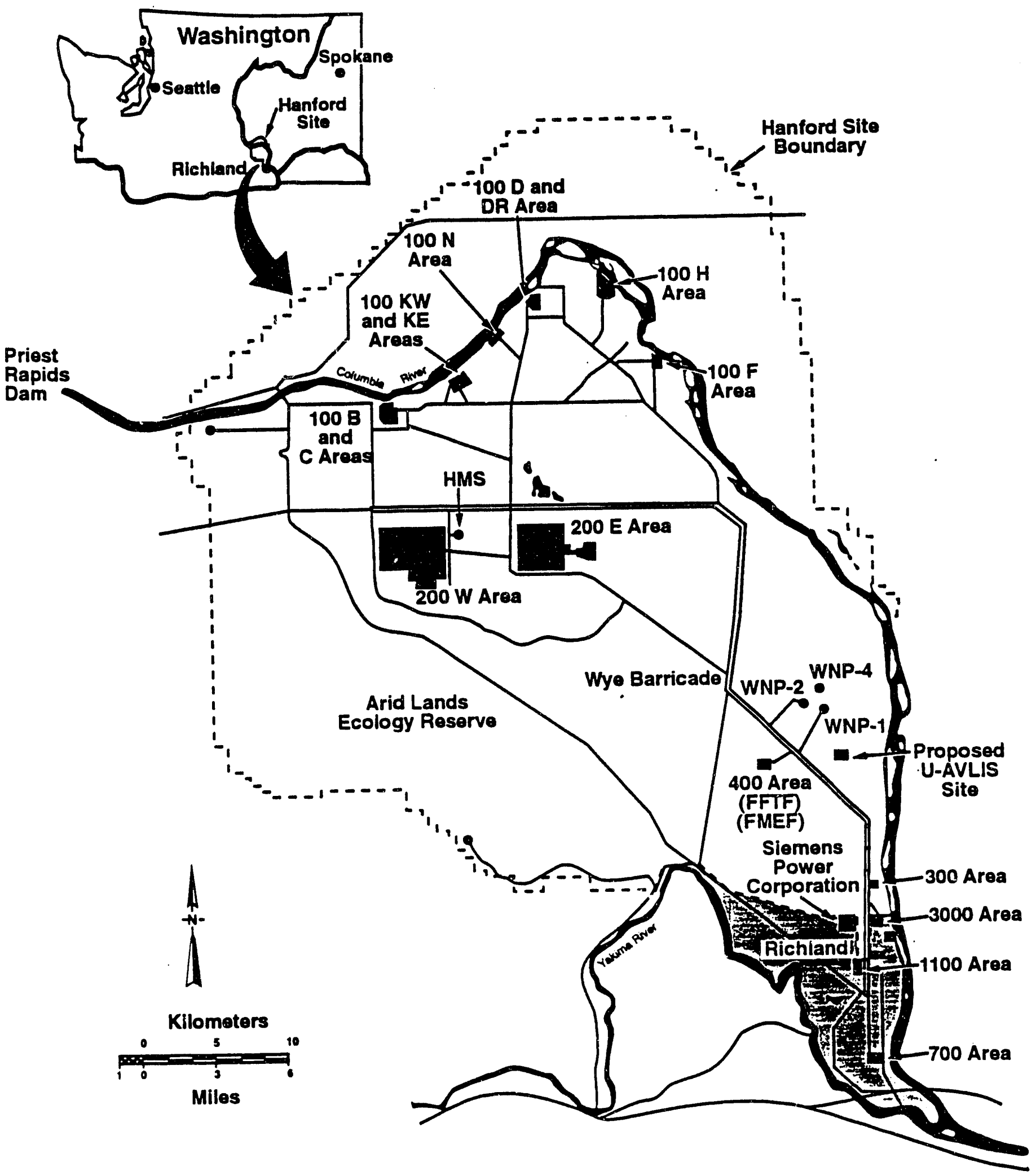


Figure 2. Hanford Site 200 West Area.

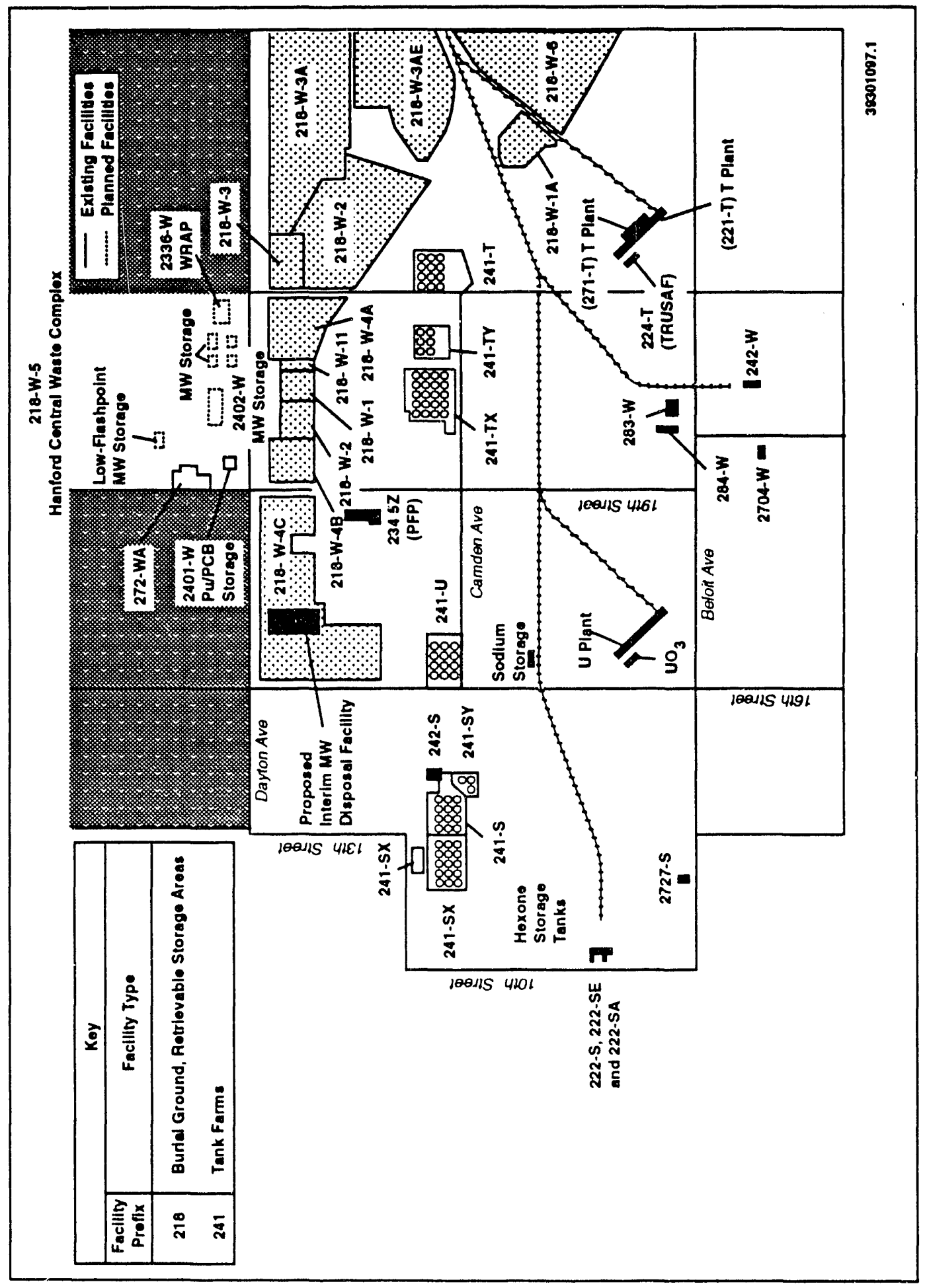


WHC-EP-0625

Figure 3. Hanford Site 200 East Area.

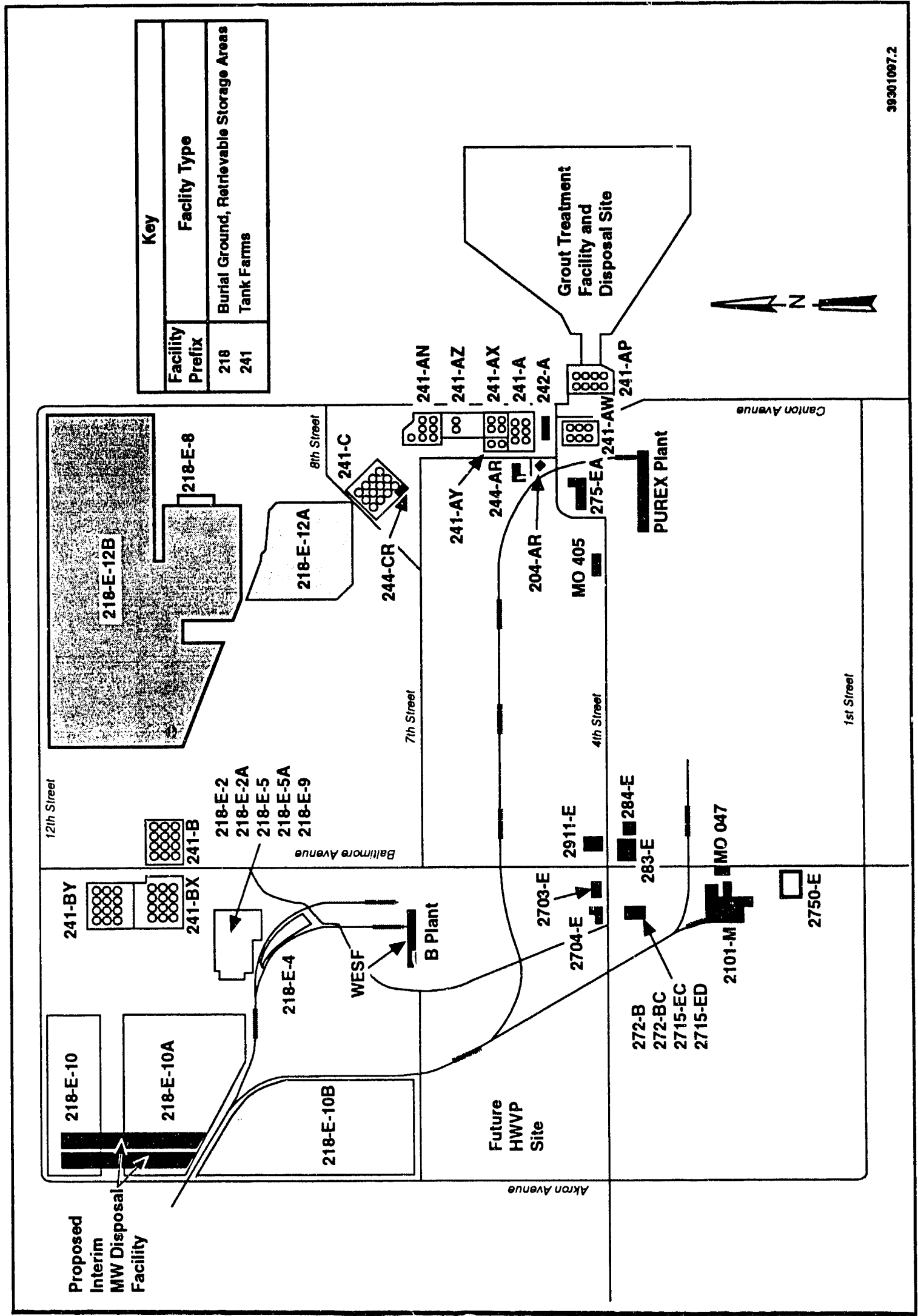


Figure 4. Single-Shell Tank.

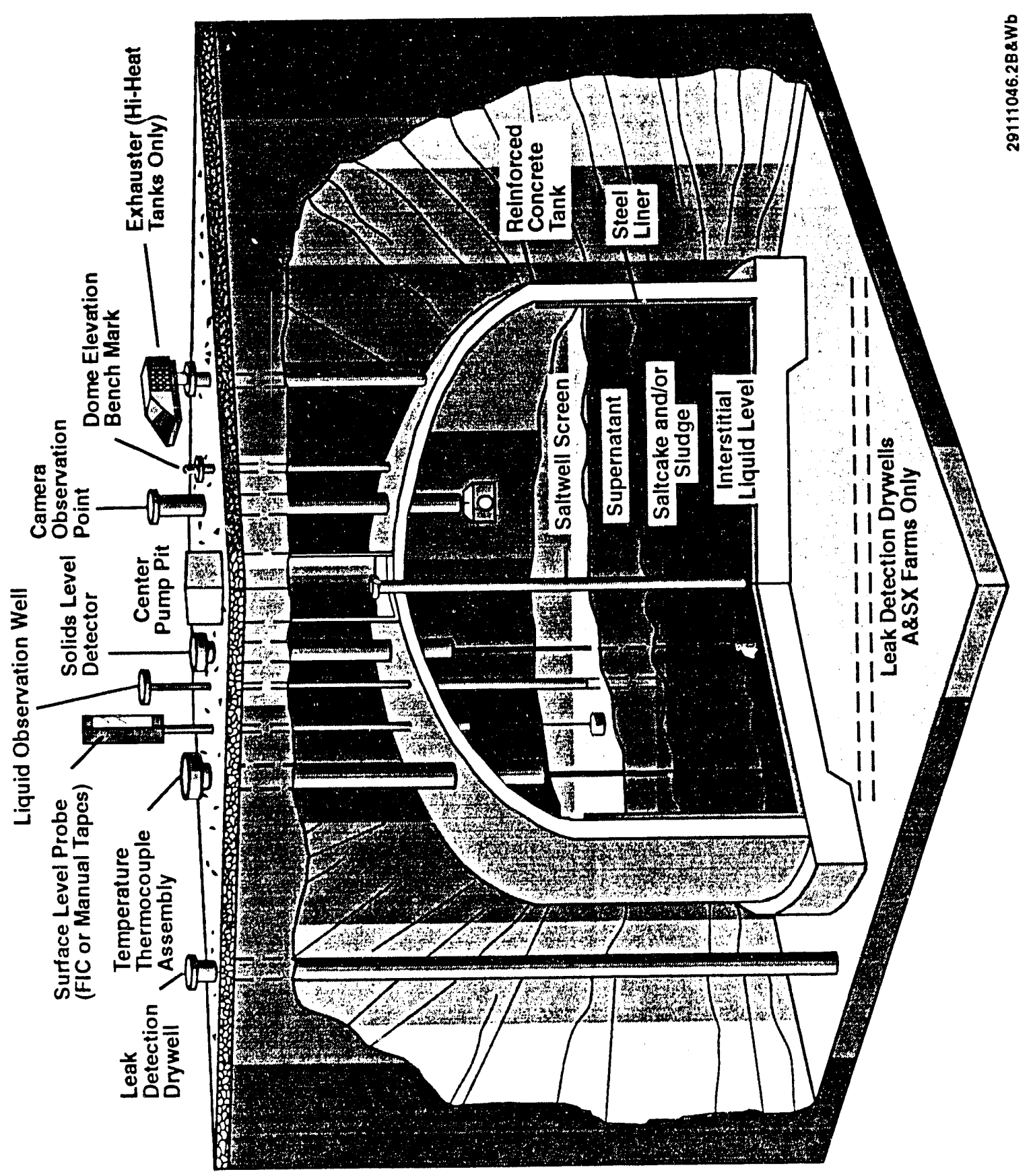


Figure 5. Double-Shell Tank.

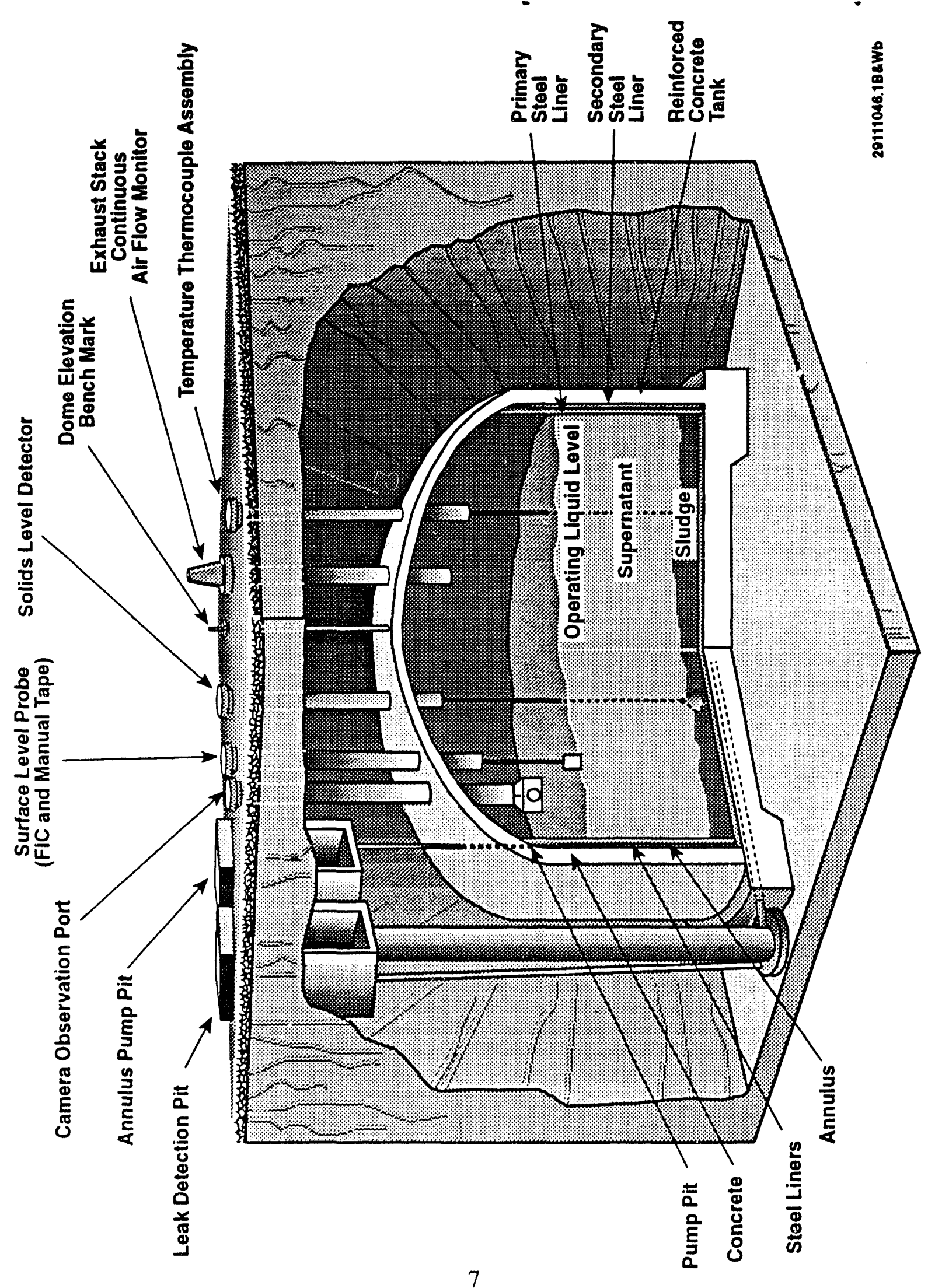


Figure 6. Tank Configuration.
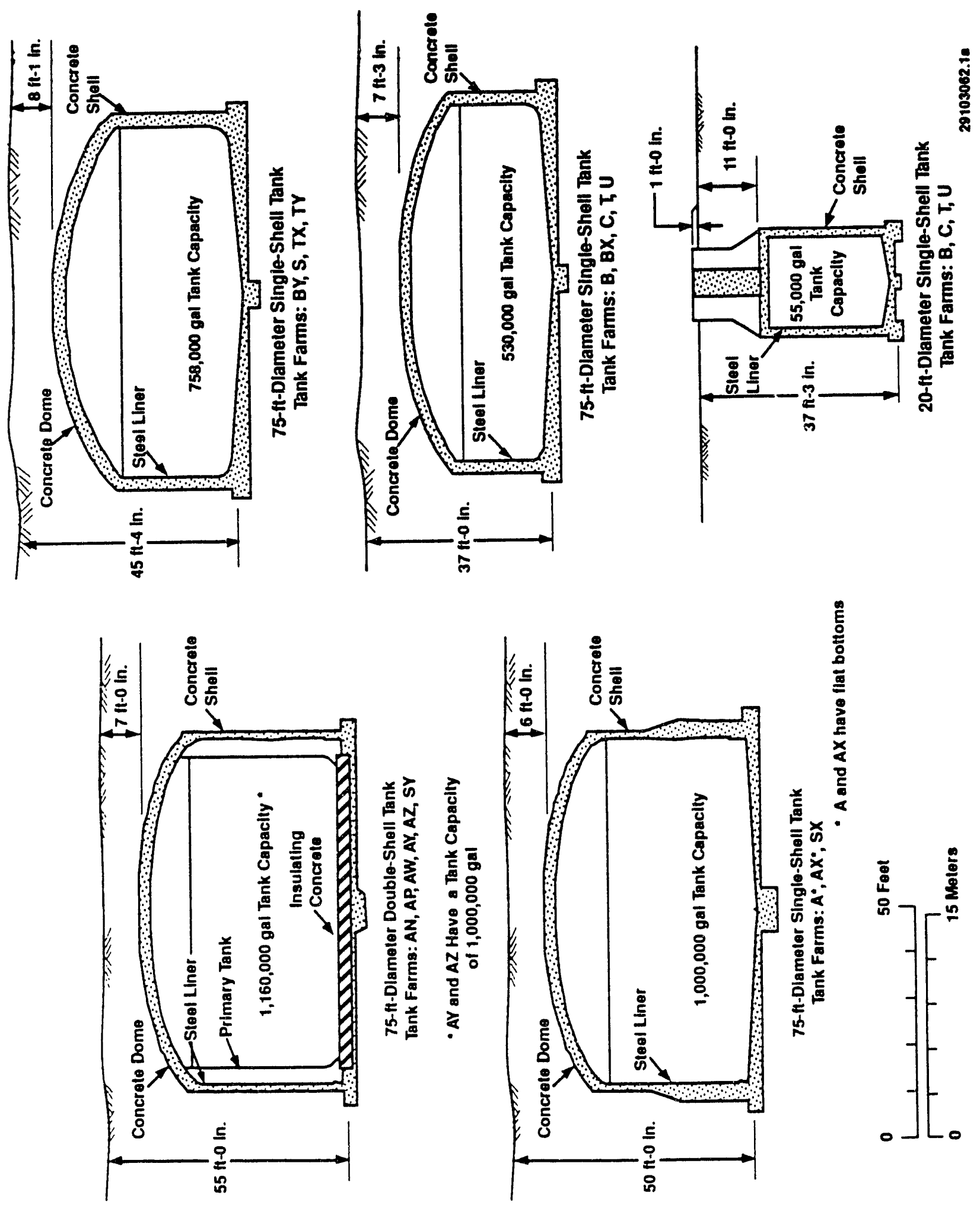


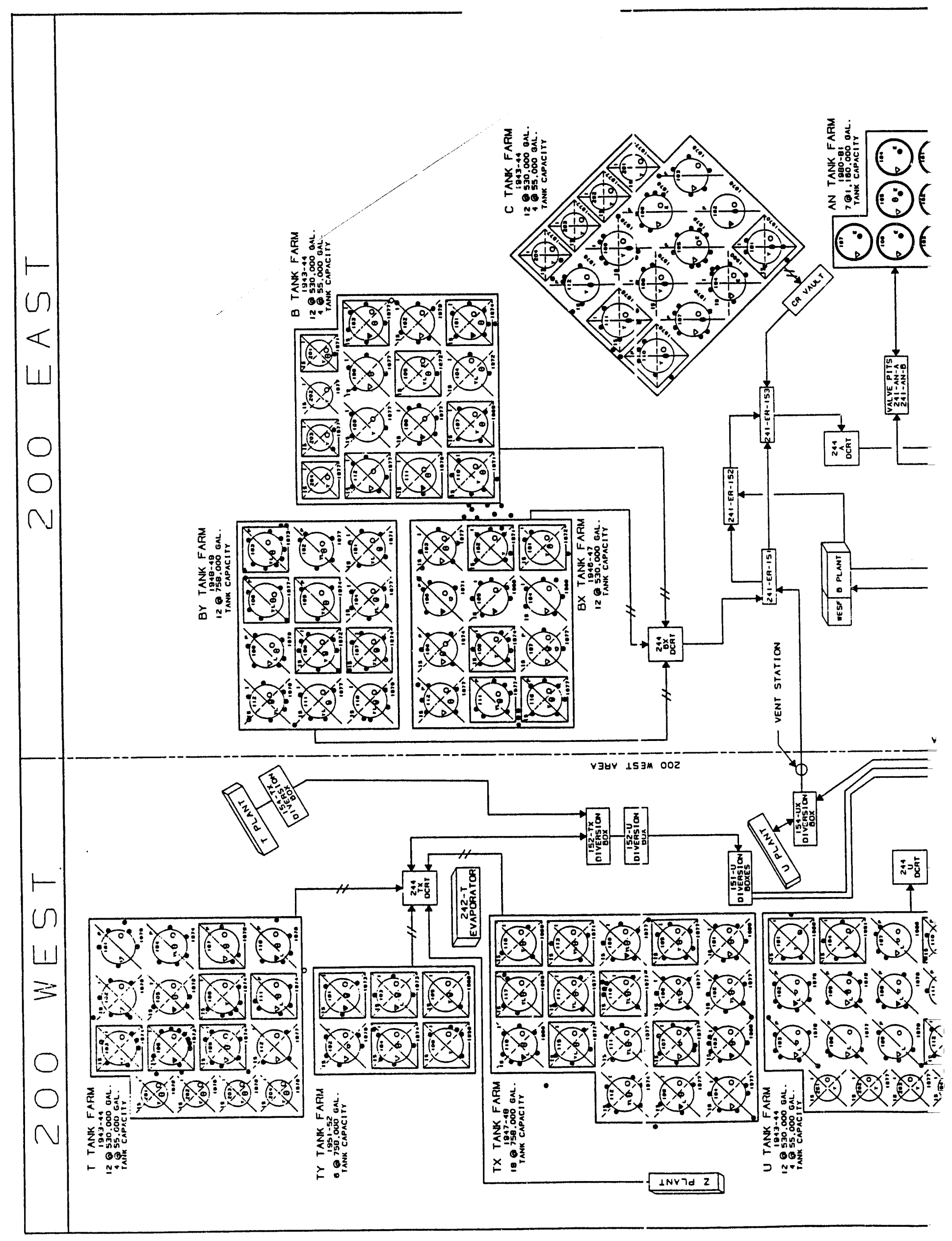




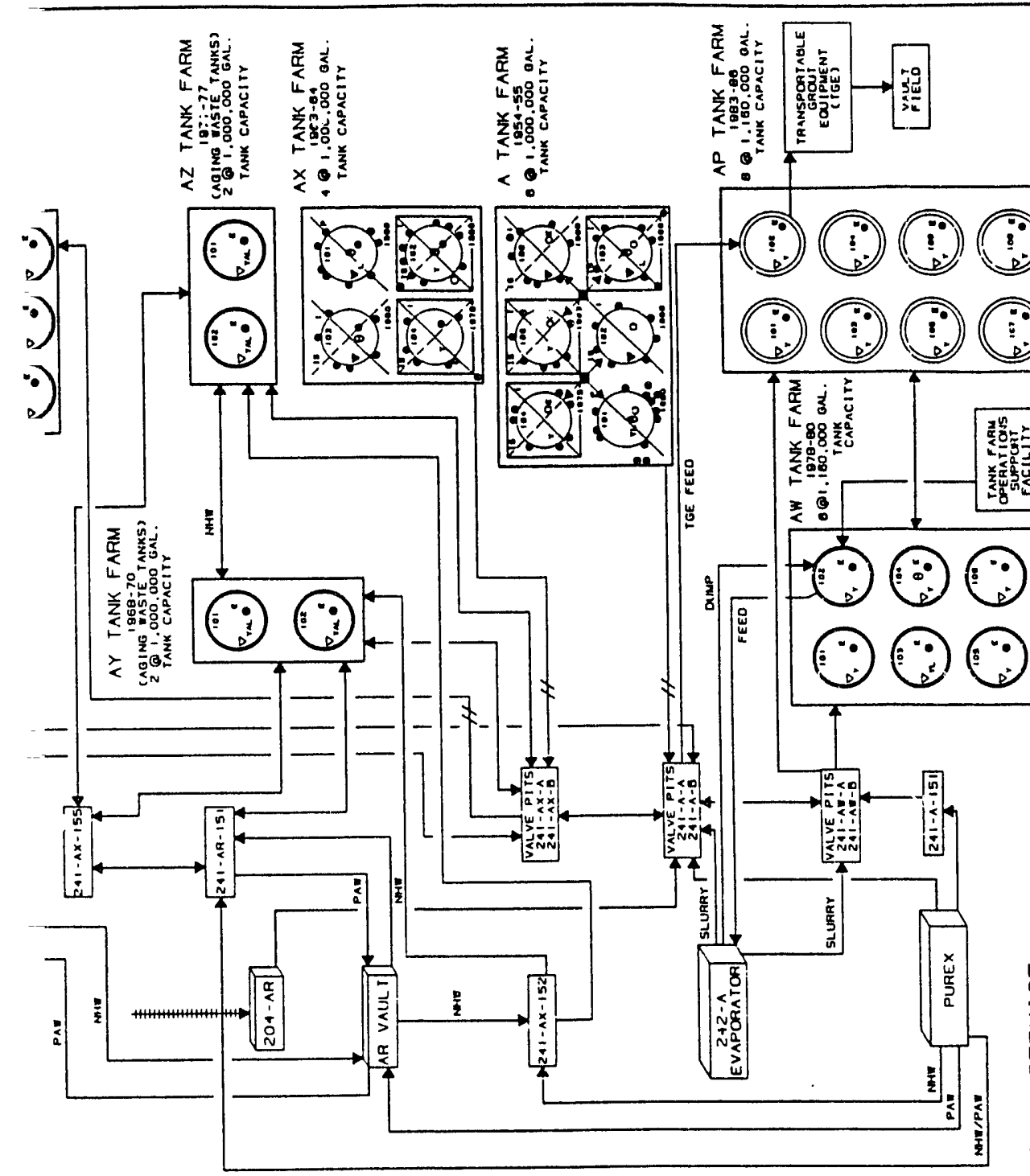

uv 15V 3002

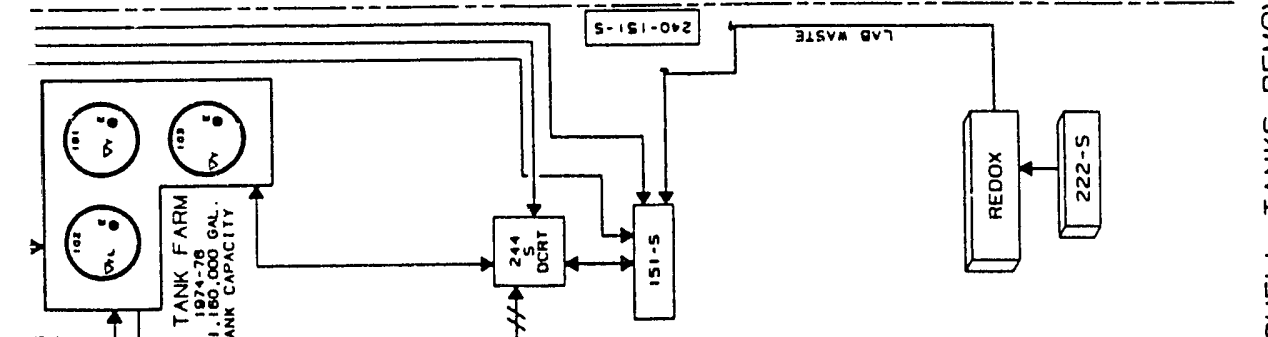

文

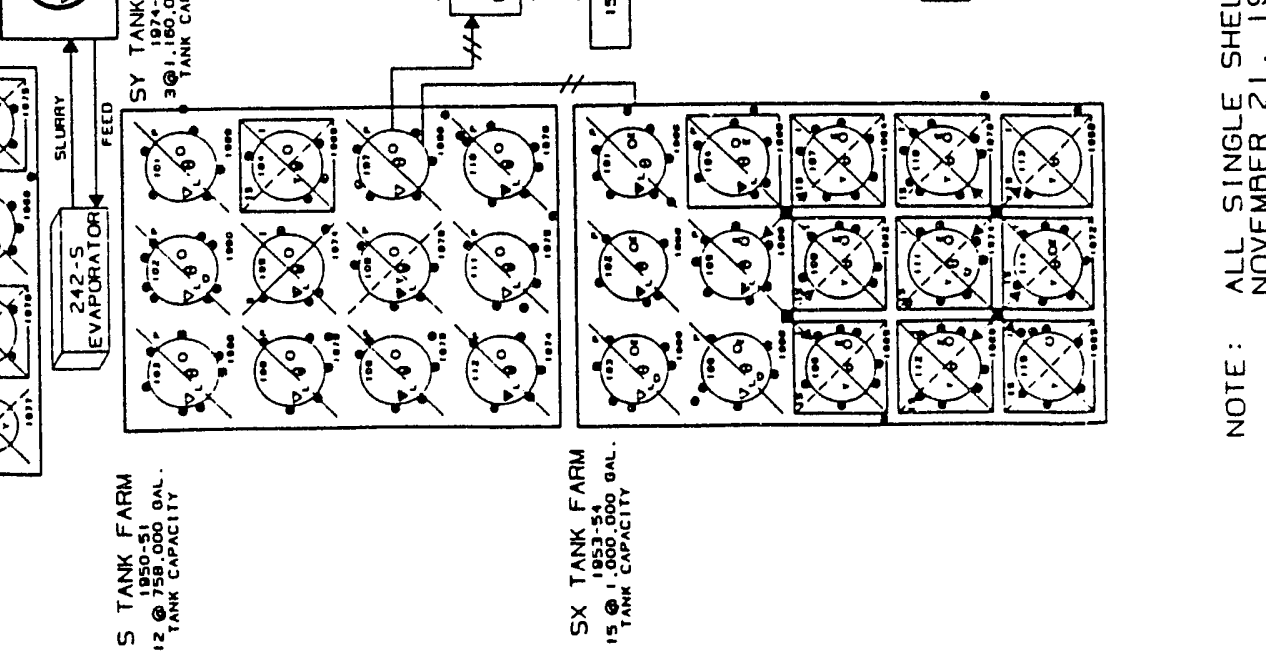

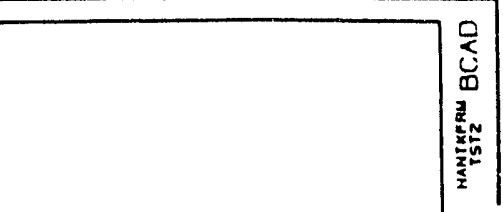

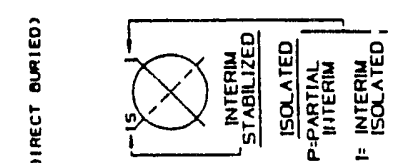

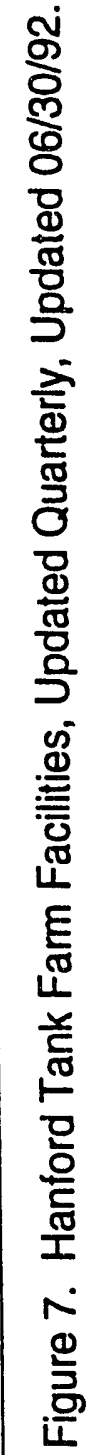




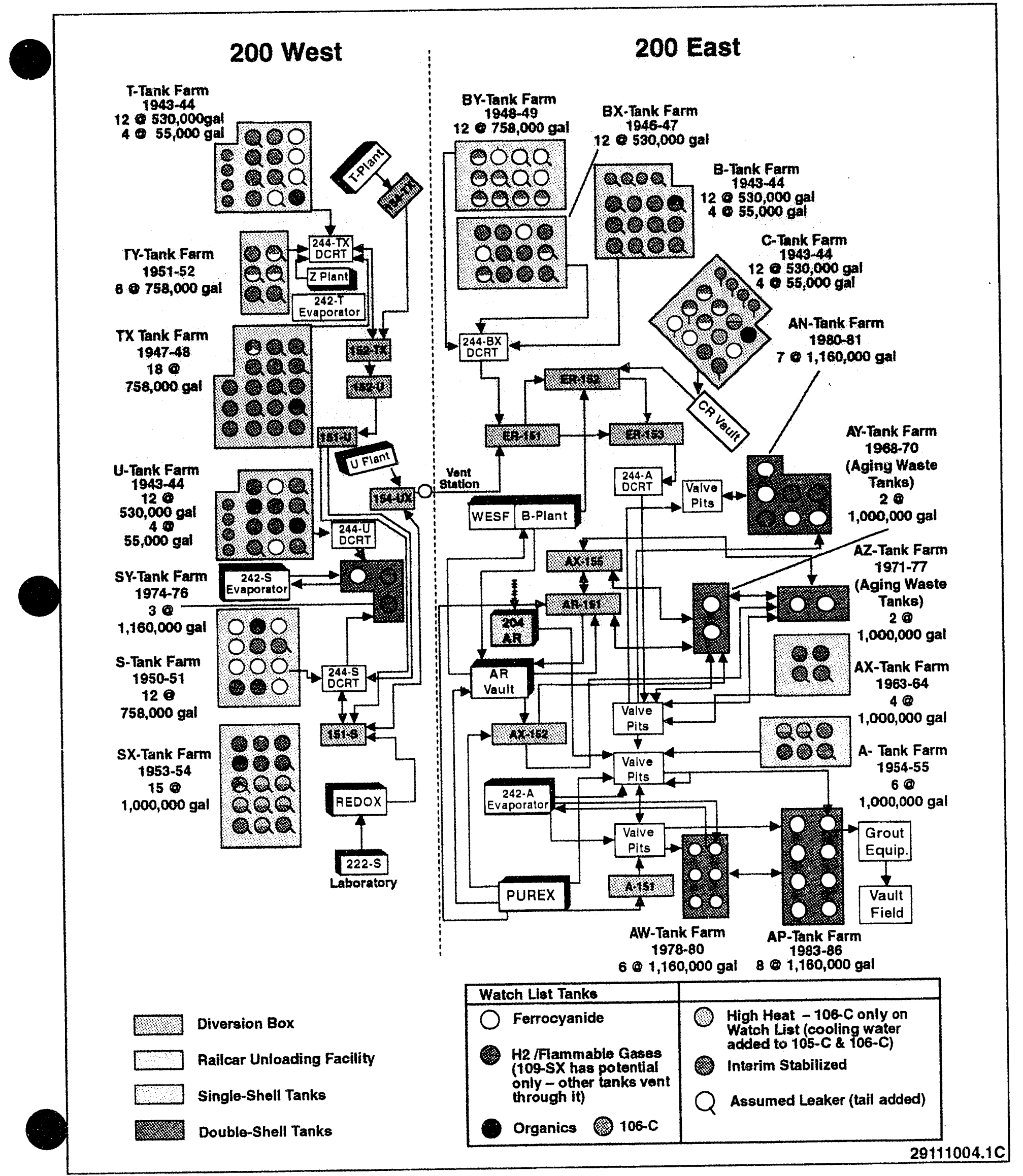

Figure 8. Watch List Tank Quick Reference, 
WHC-EP-0625

This page intentionally left blank. 


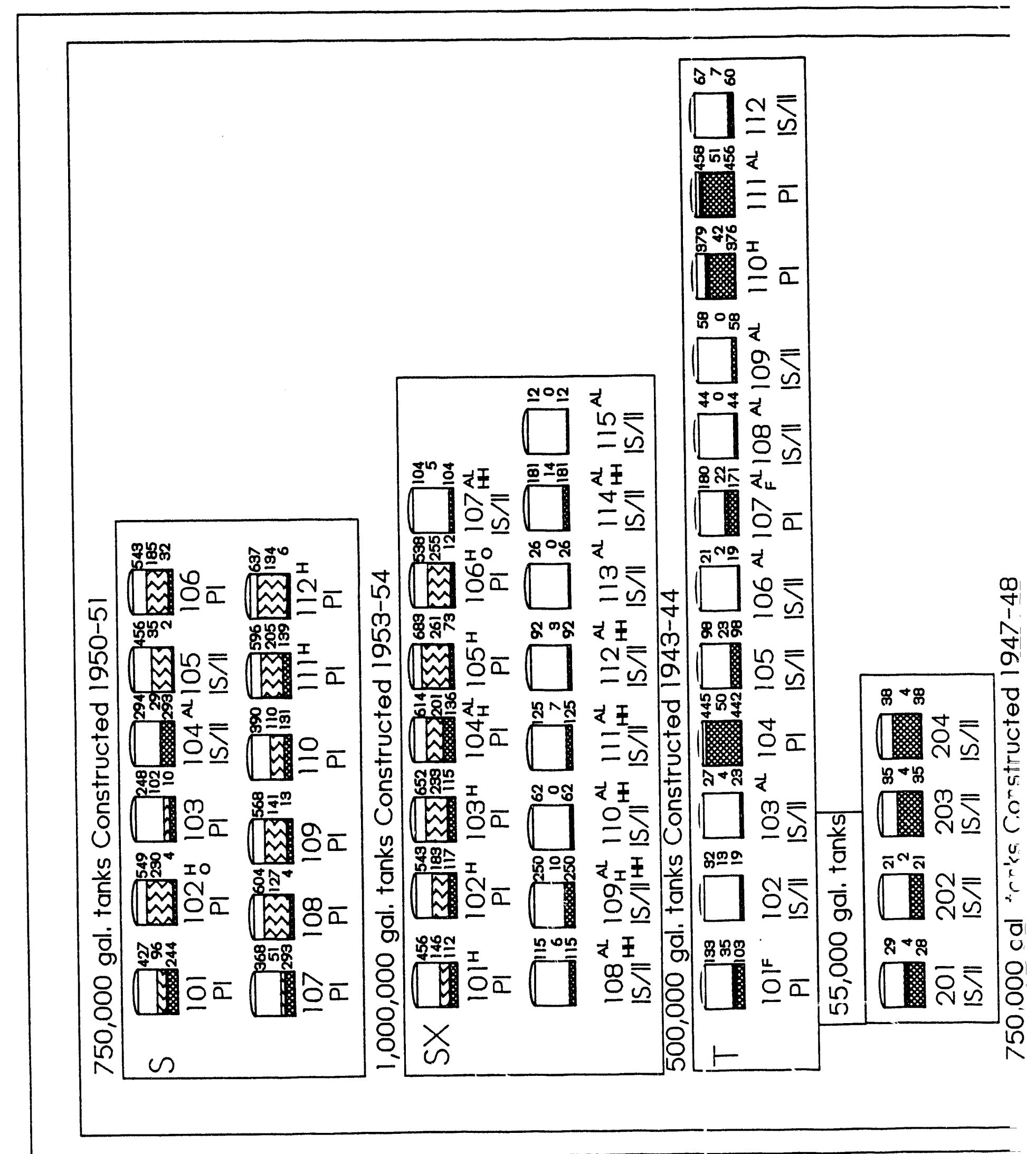



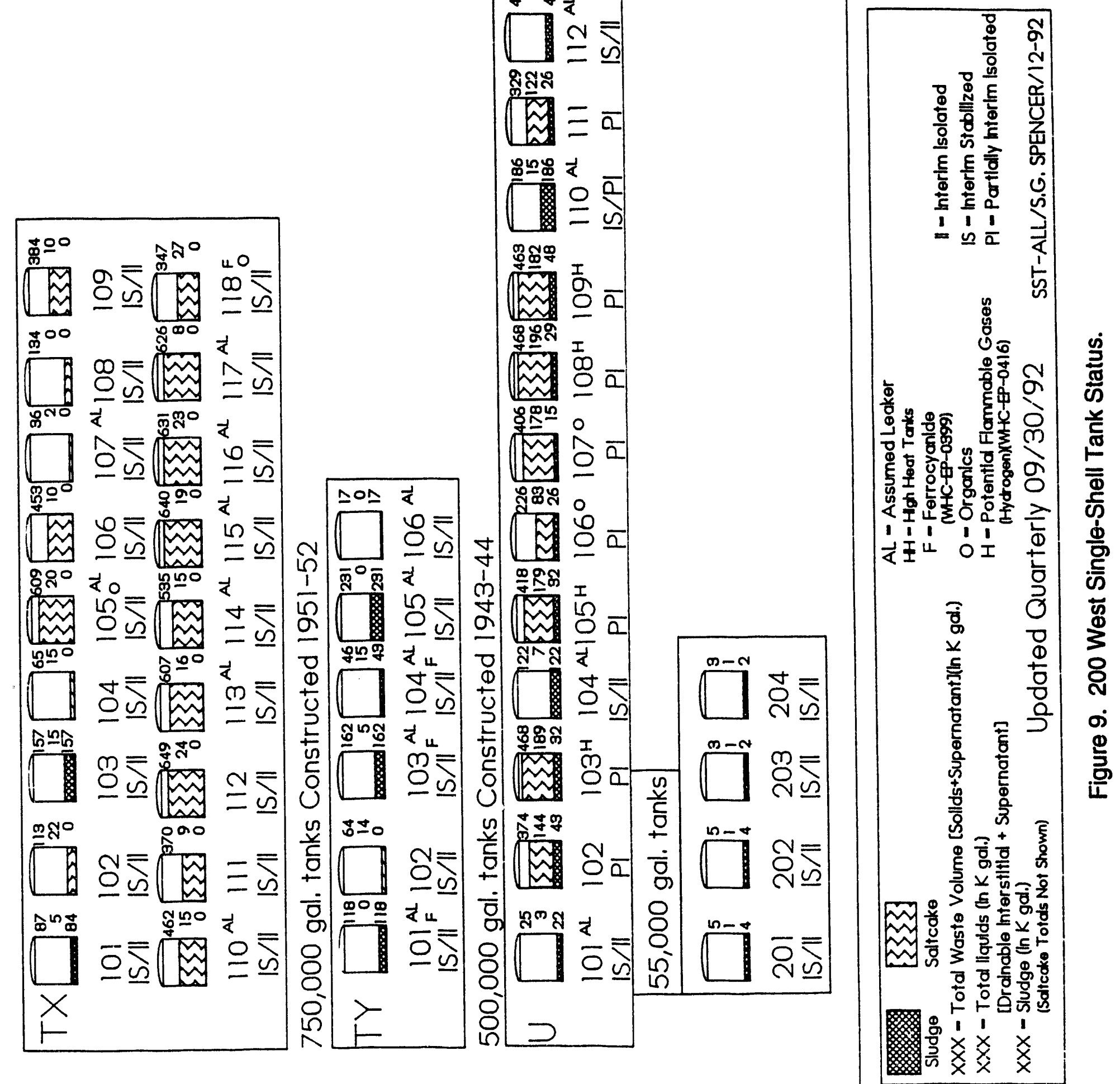


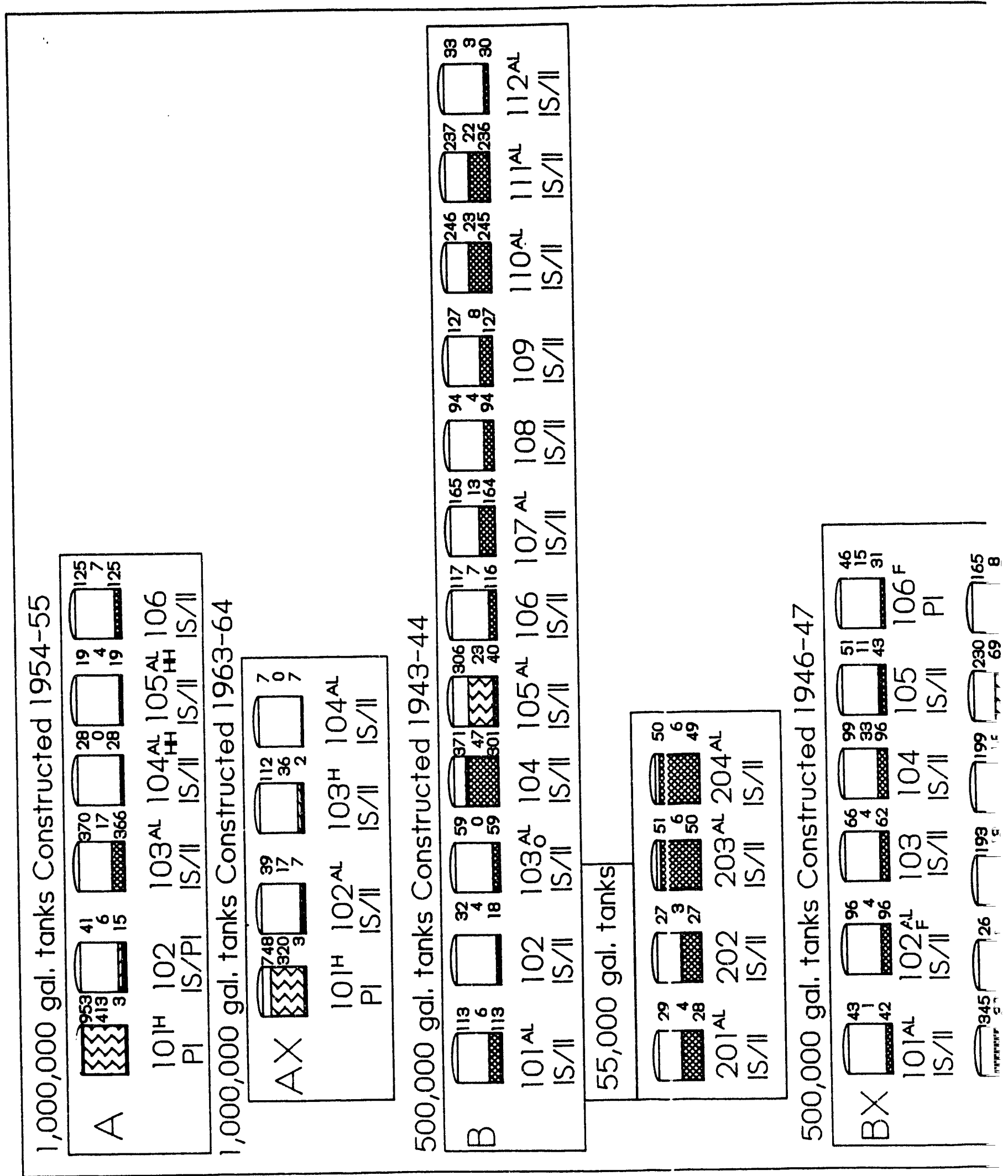




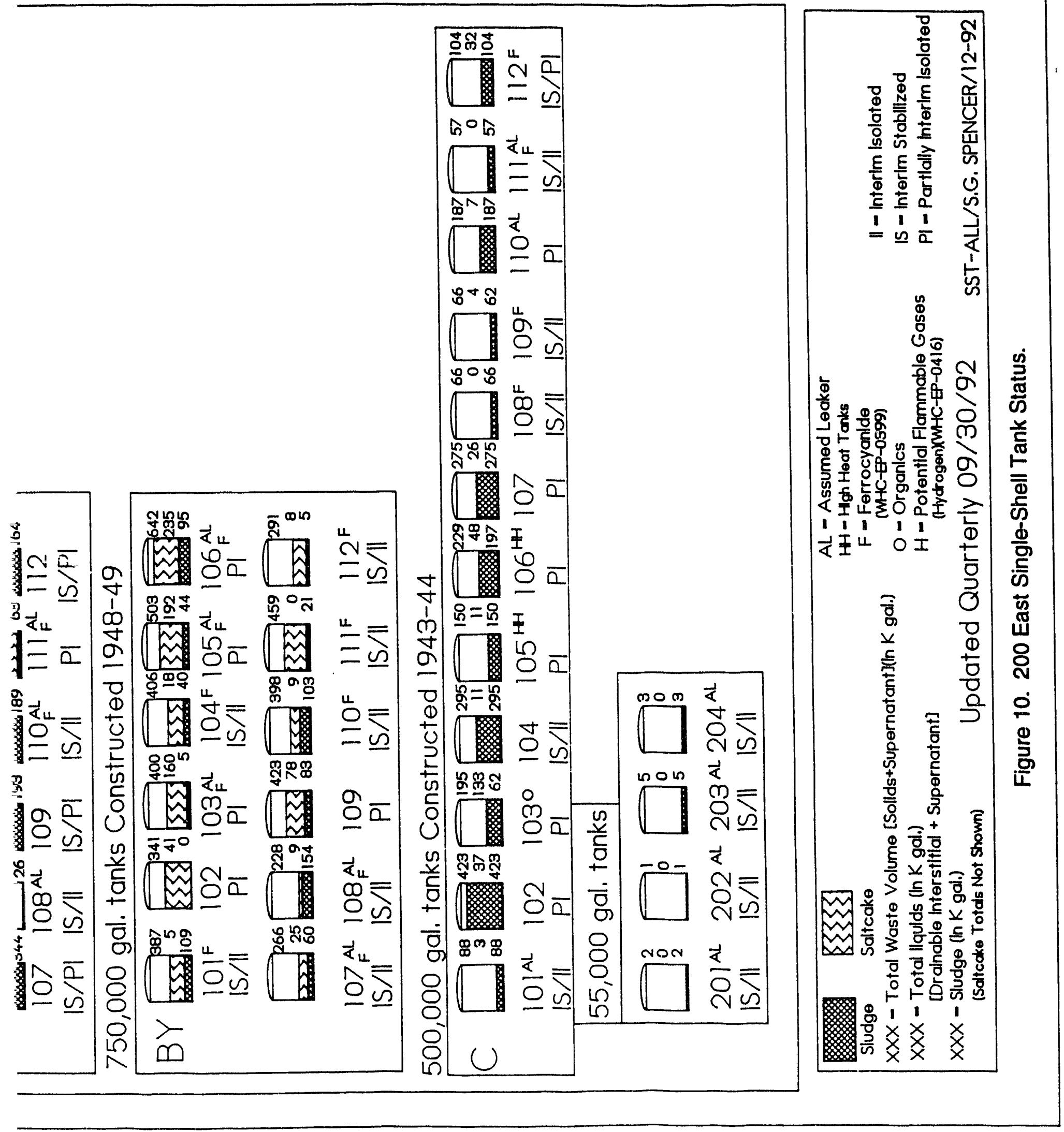


Figure 11. Double-Shell Tank Contents.

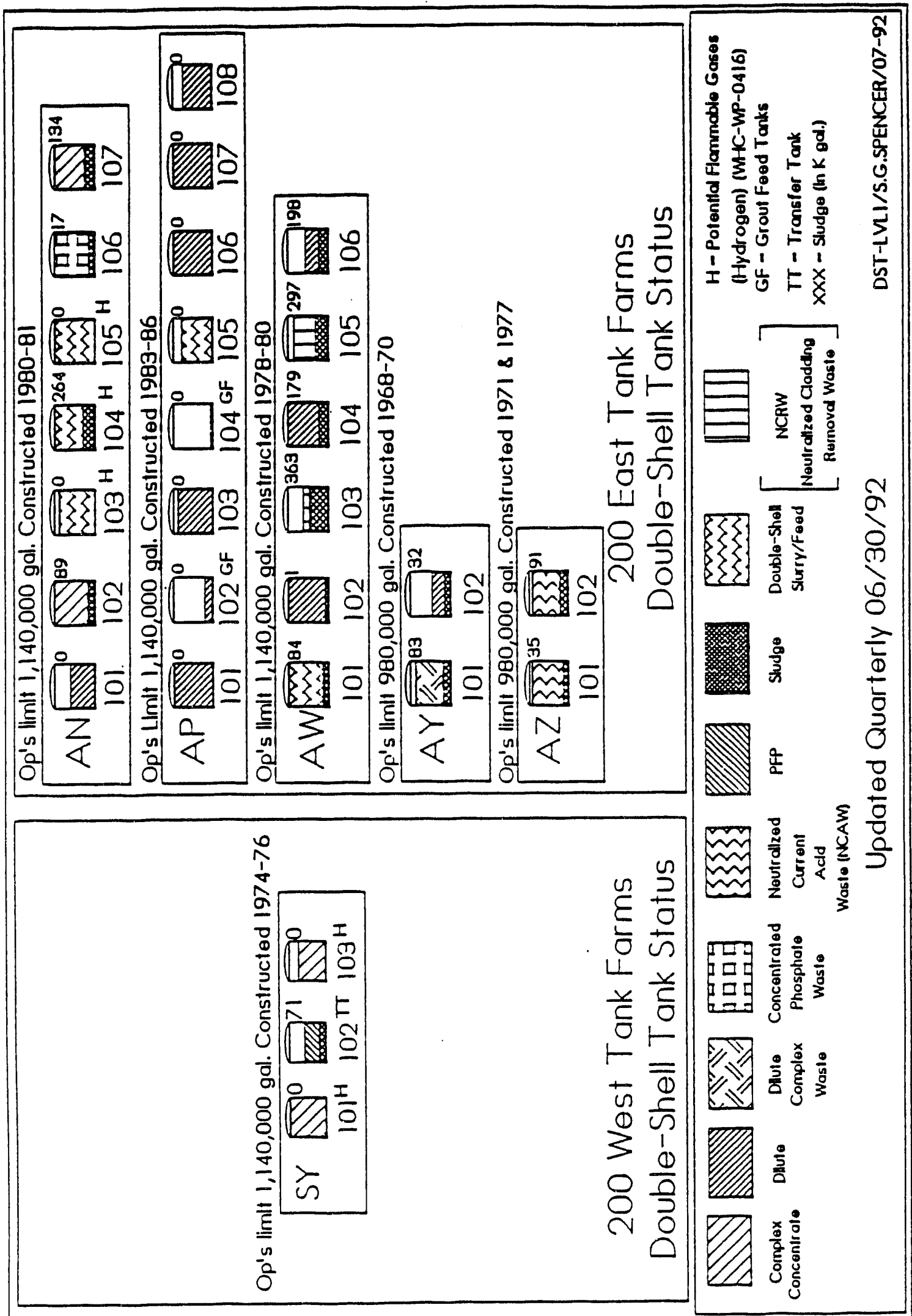


WHC-EP-0625

This page intentionally left blank. 


\section{SUMMARY TANK DATA SHEETS}

The summary data sheets cover all 161 of the Series 100 tanks. The data sheets are divided into sections based upon their location in a tank farm. Photographs are included for each tank farm and for the waste surface of each Watch List tank.

\section{SOURCES OF SUMMARY DATA}

The information presented in this document was compiled from the following sources:

- The Tank Farm Surveillance and Waste Status Summary Report for August 1992, WHC-EP-0182-54 (Hanlon 1992)

- $\quad$ The Waste Storage Tank Status and Leak Detection Criteria, "Waste Tank Data Commentaries," WHC-SD-WM-TI-356, Rev. 0, (Welty 1991)

- The surveillance database in the Surveillance Analysis Computer System (SACS), and

- Personal notes and files of the staff of the WHC Surveillance and Data Acquisition group.

\section{WATCH LIST TANKS}

A representative photo of the waste surface was chosen for each of the Watch List tanks. The view typically shows a dry waste surface, a steel tank wall, and perhaps dangling appliances, such as Food Instrument Corporation (FIC) tapes (see the photo for tank 241-AN-105). Occasionally, pools of water are visible (see photo for tank 241-AN-104), and weld seams in the steel shell are often discernable. The photo sessions included an array of shots which, when assembled, provide a panoramic view of the waste surface. The selected photo shows just one representative area of the waste surface.

\section{DEFINITION OF DATA FIELDS}

Each summary data sheet has a figure that shows riser and drywell locations and written and quantitative data for the tank description, leak detection system, and tank status. Each of these main categories is broken down into subcategories. However, each data sheet may not have information for every possible subsection because certain data may not be applicable to that tank. For example, if a tank has no manual tape surface-level instrument, then the heading for that measurement data is not included. The following paragraphs define the elements within each main heading on the tank data sheets. 


\section{RISER AND DRYWELL LOCATIONS}

The figure shows the relative positions of only those risers that contain surface-level detection devices (manual tape, radar, or FIC) and liquid observation wells (LOW). Other risers are not shown. These drawings are not to scale. Refer to Figure 7 in the previous section for nearby drywells primarily associated with another tank.

\section{TANK DESCRIPTION}

Type. Tank type may be either SST or DST. These types are depicted in Figures 4 and 5 in the previous section.

Constructed. The date construction on the tank was completed.

In-service. The date liquids were first added to the tank.

Out of Service. The date the tank was declared unavailable to receive any additional waste. All single-shell tanks were declared out of service no later than November 21, 1980. If an out-of-service date is not listed, then the November 21, 1980 date applies.

Diameter. This is the measurement in feet of the inside diameter of the liner. All the 100 Series tanks are 75 feet in diameter, resulting in the conversion factor of 2,750 gallons per inch of depth.

Usable Depth. This is simply the rated capacity divided by the number of gallons per foot. This is not the maximum operating height, nor is it derated for any change in condition of the tank (such as an assumed leak).

Capacity. Rated capacity at construction. This is not derated for any condition of the tank (such as an assumed leak).

Bottom Shape. This can be dish or flat. The dished bottom tank has a slope that leads down toward the center of the tank. The dish typically occupies a volume of 12,500 gallons.

Hanford Coordinates. This is the tank's location on the site and is measured in feet (offsets) from a reference point on the site. This method of location was chosen (in lieu of latitude and longitude) as a security measure during World War II.

Air Lift Circulators. These devices were once used to agitate tank contents by bubbling air up through them. Most tanks do not have these devices.

Ventilation. Active ventilation indicates that a powered exhauster draws air from the space over a tank through a high-efficiency particulate air (HEPA) filter and vents it out to the environment. This puts the space above a tank at a negative pressure (relative to ambient), 
minimizing the potential for release of airborne contaminants to the environment. Passive ventilation means that a breather tube connects the spaces above the tank with the environment through a HEPA filter. Winds and barometric (weather) changes will cause air to move in and out of the tank, primarily through the filters.

\section{LEAK DETECTION SYSTEM}

FIC Riser. This entry lists the riser number through which the FIC (if any) is lowered. If this information is missing from a tank data sheet, the tank does not currently contain an FIC device. FIC, a vendor who supplied the automated level-measuring device. A plummet is lowered under machine control until electrical conductivity is established with the waste surface.

Manual Tape Riser. This entry lists the riser through which the manual tape is lowered. If this entry is missing on a data sheet, the tank does not currently contain a manual tape device. A manual tape is a surface-level sensing device that requires an operator to manipulate it. A plummet is lowered by hand crank until electrical conductivity is established with the waste surface.

LOW Riser(s). The riser numbers for the LOWs are listed under this entry. The LOW is a sealed tube that extends down into the waste. Sensors are lowered into the tube to detect radiation level or moisture. This is an especially important technique when a tank has been pumped and thus has a dry surface. This method of water detection gives a glimpse beneath the surface.

Number of External Drywells. This entry reports the number of wells present. Drywells are cased holes drilled adjacent to a waste tank. Instruments are lowered to detect radiation level or moisture. The figure on each tank data sheet depicts the drywell's relative position around the tank. Drywells are numbered by "clock position" with 12 o'clock referring to true north.

A drywell identified as 06 (6 o'clock) would be due south of the center of the tank. There are no drywells adjacent to any DSTs.

\section{TANK STATUS}

Watch List. This entry indicates the reason why this tank is on the Watch List. If this entry is not listed for a tank, then the tank is not on the Watch List. The four Watch List categories are: ferrocyanide, hydrogen/flammable gas potential, high-heat, and organic salt. These are identified as Priority 1 Hanford Site Tank Farm Safety Issues in Public Law 101-510, Section 3137, "Safety Measures for Waste Tanks at Hanford Nuclear Reservation." 


\section{Contents}

Type. The source of the waste, such as Plutonium-Uranium Extraction (PUREX) decladding, or its content, such as dilute complexed waste, is described here. More complete definitions of these terms are found in the Glossary.

Total Waste. This is the sum of the solids volume and the supernatant liquid reported in WHC-EP-0182 (Hanlon 1992). This is a somewhat peculiar number, because its definition leads to apparent discrepancies with an estimated volume based on the reported surface level. The number reported as total waste is not updated, except as the result of a tank investigation or a pumping campaign. Evaporation and other processes may lead to apparent discrepancies as well.

Supernatant Volume. This is the amount of liquid found in standing pool(s) on top of solids. This figure is derived from numbers found in WHC-EP-0182 (drainable liquid remaining minus drainable interstitial). Supernate is usually calculated by subtracting the solids-level measurement from the liquid-level measurement and multiplying by the appropriate conversion factor to get gallons $(2,750$ gallons/inch for the 75 -foot diameter tanks).

Drainable Interstitial. This figure represents liquid believed to be held in pores in the solid waste, but which is expected to be free to drain, if pumped. This is usually calculated based on salt cake and sludge volumes, using average porosity values or actual porosity data for a tank, when available.

\section{Isolation Status}

Some isolation dates were not easily accesible. If a tank was known to be isolated, but no date was immediately available, then only the isolation date was omitted. If a tank was not isolated, the entire reference to an isolation date was excluded from the data sheet.

Date Interim Stabilized. If this entry is not present for a tank, it has not been interim stabilized. Interim stabilized means that the tank has been pumped and meets certain stabilization criteria, such as having less than 50,000 gallons of drainable interstitial liquid and less than 5,000 gallons of supernatant. If the tank was jet pumped to achieve stabilization, then the jet pump flow must have been at or below $0.5 \mathrm{gal} / \mathrm{m}$ before interim stabilization criteria were met.

Date Partially Interim Isolated. If this entry is not present for a tank, it has not been partially interim isolated. This is the administrative designation reflecting the completion of the physical effort required for interim isolation, except that all isolation of risers and piping required for jet pumping may not be completed. 
Date Interim Isolated. If this entry is not present for a tank, it has not been interim isolated. This is the administrative designation reflecting the completion of the physical effort required to minimize the addition of liquids into an inactive storage tank.

\section{Surface Level/Leak Status}

Integrity Category. This may be sound or an assumed leaker. Sound indicates no loss of liquid attributed to a breach of integrity.

Date Declared Confirmed or Assumed Leaker. If this entry is not present for a tank, it has not been declared an assumed leaker. In many cases, a leak was suspected long before it was identified or confirmed. In 1984, the criteria designations of "suspected leaker," "questionable integrity," and "confirmed leaker" were merged into one category, now reported as an "assumed leaker."

Leak Volume Estimate. If this entry is not present for a tank, it has not been declared an assumed leaker. For more complete information, refer to WHC-EP-0182, Appendix H, Leak Volume Estimates (Hanlon 1993).

Manual Tape Surface Level. The level is reported in inches, along with the date of measurement. If this entry is not present, the tank does not have a manual tape device installed. This is an electrical conductivity-based device for determining the surface level of a tank. On some tanks, these measurements are taken as often as daily.

FIC Surface Level. The level is reported in inches, along with the date of measurement. If this entry is not present, it does not have an FIC device installed. This is an electrical conductivity-based device for determining the surface level of a tank. These measurements are taken daily.

Surface Level Comments. These comments may indicate if an FIC is in intrusion mode or is out of service.

Last Photographed. The date the tank was last photographed. If this field is not present, the tank interior has not been photographed.

Photo Interpretation Comments. Comments on those photos will be included in a future document release.

\section{Temperature Status}

Highest Temperature during 1992. This figure is reported in degrees Fahrenheit. Some tanks were not measured in 1992. In those cases we attempted to find the most current 
reading. Some temperature data were not easily accesible. The temperature reading field was left blank when useful data were not found in time for inclusion with this issue.

Comments. This includes any special information about temperature status.

\section{Drywell Status}

Comments. If this field is not listed on a data sheet, the tank does not have any drywells to monitor (most likely a DST). Drywell profiles are compared with established baselines to determine if changes have taken place, which may indicate a tank may be leaking. Drywell profiles are plots of radioactivity versus depth. Low levels of activity indicate only background radiation is present. Elevated counts mean that radioactive materials have leaked into the ground and spread near the drywell being monitored. Most single-shell tanks have at least four drywells near their periphery. No DST's have drywells. 





WHC-EP-0625

This page intentionally left blank. 


\section{Tank 241-A-101}

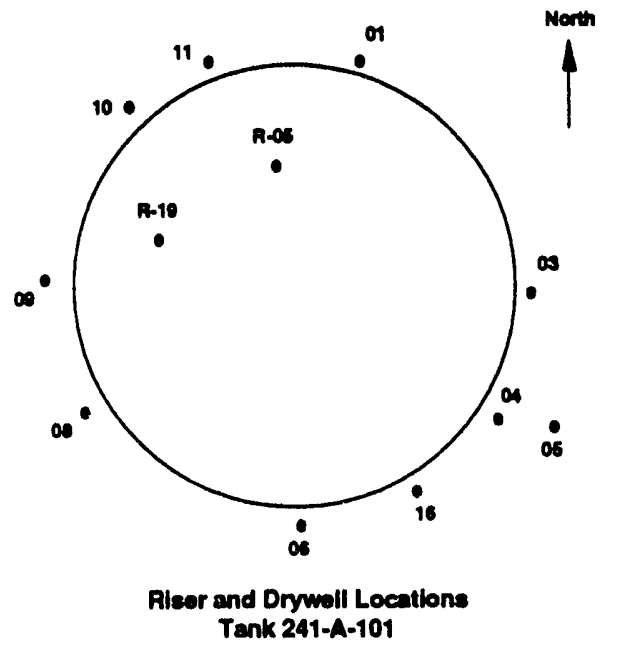

Leak Detection System

Surface Level: FIC Riser- None

Manual Tape Riser- R-05

LOW Riser(s)- R-19

Number of External Drywells: 10

Number of Lateral Wells: 3

\section{Tank Status}

Watch List: Hydrogen

Contents

Type: Double Shell Slurry Feed

Total Waste: 953K gallons

Supernate Volume: OK gallons

Drainable Interstitial Liquid: $413 \mathrm{~K}$ gallons

Isolation Status

Date Partially Interim Isolated: 12/15/82

Surface Level/Leak Status

Integrity Category: Sound

Manual Tape Surface Level: 345.25 Inches (12/28/92)

Last Photographed: $08 / 21 / 85$

Photo Interpretation Comments: See attached photo.

Temperature Status

Highest temperature during 1992: $162{ }^{\circ} \mathrm{F}(08 / 06 / 92)$

\section{Drywell Status}

Comments: Temperatures are stable.

Comments: Current drywell profiles were stable and consistent with established baseline profiles.
Tank Description

Type: Single Shell

In-service: $01 / 24 / 56$

Out of Service: 11/21/80

Diameter: 75'

Usable Depth: 30.3'

Capacity: $1,000 \mathrm{~K}$ gallons

Bottom shape: Flat

Hanford Coordinates:

$41,205^{\prime}$ North

47,804' West

Air Lift Circulators: Yes (4)

Ventilation: Passive
Constructed: 1955 
WHC-EP-0625

This page intentionally left blank. 


\section{Tank: 241-A-101}

\section{August 1985}

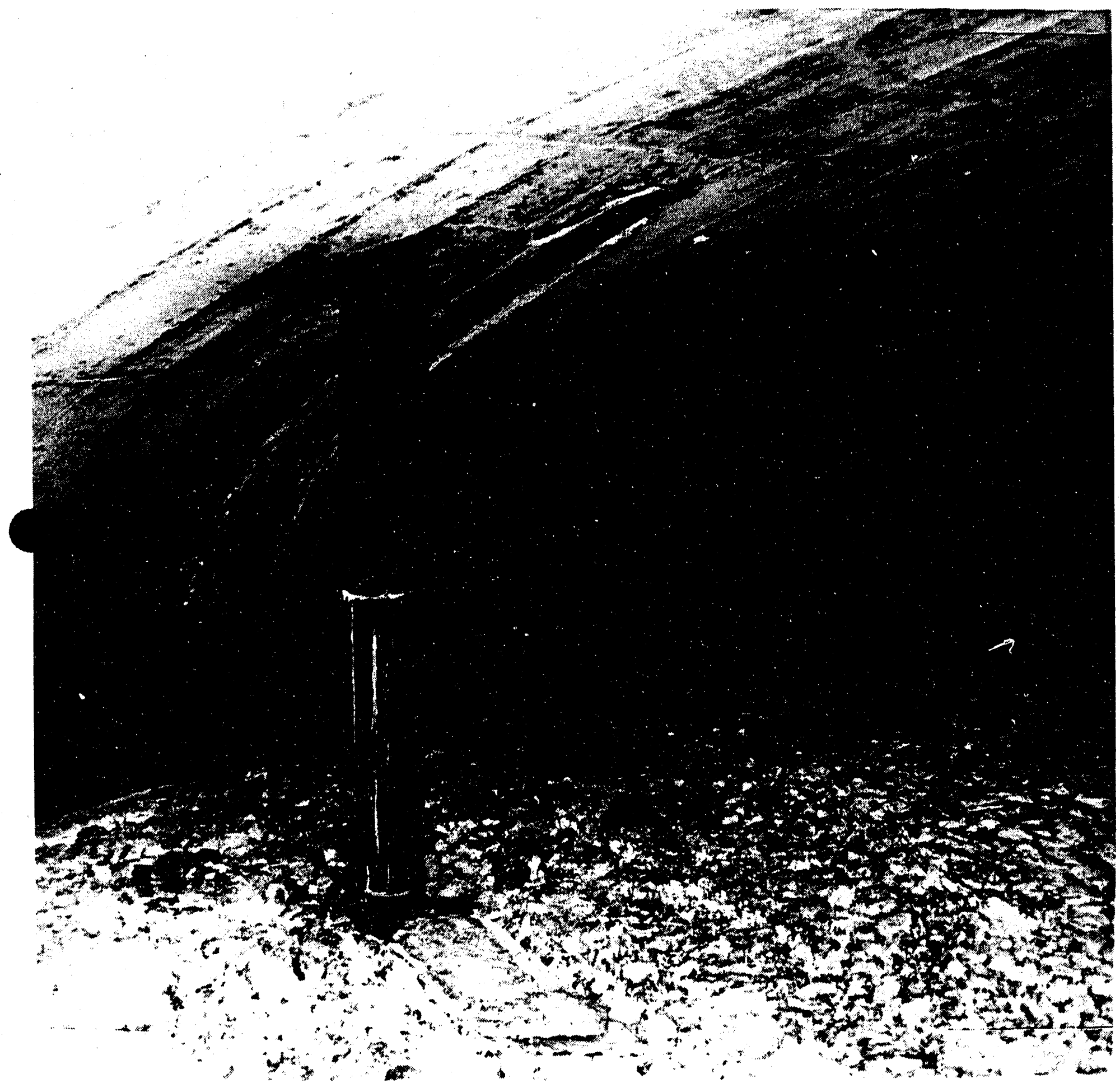


WHC-EP-0625

This page intentionally left blank. 


\section{Tank 241-A-102}

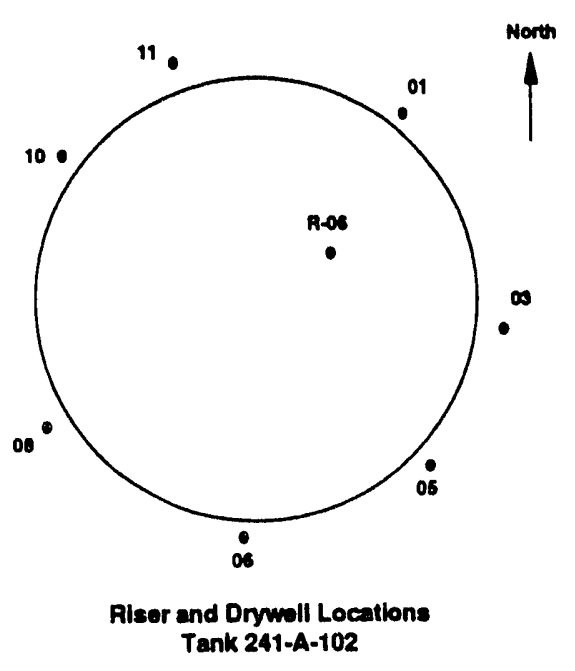

Number of External Drywells: 7

Number of Lateral Wells: 3

\section{Tank Status}

Contents

Type: Double Shell Slurry Feed

Total Waste: $41 \mathrm{~K}$ gallons

Supernate Volume: 4K gallons

Drainable Interstitial Liquid: $2 \mathrm{~K}$ gallons

Isolation Status

Date Interim Stabilized: $08 / 89$

Date Interim Isolated: $12 / 15 / 82$

\section{Surface Level/Leak Status}

Integnity Category: Sound

FIC Surface Level: Intrusion level set at 14.00 Inches $(12 / 28 / 92)$

Surface Level Comments: Intrusion Mode

Last Photographed: 07/20/89

Photo Interpretation Comments:

Photos reveal a surface of clear liquid and large areas of what appear to be a relatively thin layer of floating solids. The FIC plummet is not readily visible, but appears to be contacting solids.

\section{Temperature Status}

Highest temperature during 1992: $91.9^{\circ} \mathrm{F}(01 / 01 / 92)$

Comments: Temperatures are stable.

Drywell Status

Comments: Current drywell profiles were stable and consistent with established baseline profiles. 
WHC-EP-0625

This page intentionally left blank. 


\section{Tank 241-A-103}

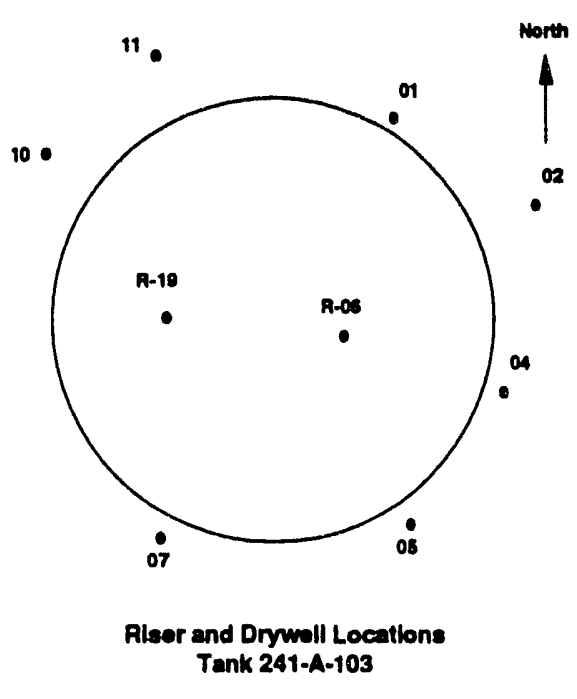

Number of External Drywells: 7

Number of Lateral Wells: 3

\section{Tank Status}

Contents

Type: Double Shell Slurry Feed

Total Waste: $370 \mathrm{~K}$ gallons

Supernate Volume: $4 \mathrm{~K}$ gallons

Drainable Interstitial Liquid: 13K gallons

Isolation Status

Date Interim Stabilized: $08 / 88$

Date Partially Interim Isolated: $12 / 15 / 82$

Surface Level/Leak Status

Integnty Category: Assumed Leaker

Date Declared Confirmed or Assumed Leaker: 1987

Leak Volume Estimate: 5.500 Gallons (1987)

FIC Surface Level: Intrusion level set at 137.40 Inches $(12 / 28 / 92)$

Surface Level Comments: Intrusion Mode

Last Photographed: 12/28/88

Photo Interpretation Comments:

Photos reveal a liquid surface with exposed solids (some floating) in many areas.

\section{Temperature Status}

The FIC plummet is contacting liquid with exposed solids near the plummet.

Highest temperature during 1992: $118^{\circ} \mathrm{F}(04 / 09 / 92)$

Drywell Status

Comments: Temperatures are stable.

Comments: Current drywell proflles were stable and consistent with established baseline profiles. 
WHC-EP-0625

This page intentionally left blank. 


\section{Tank 241-A-104}

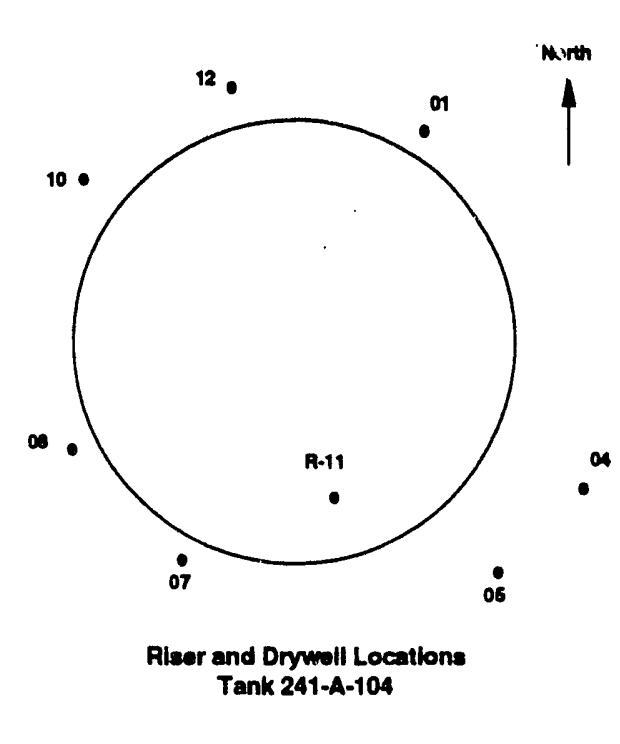

Number of External Drywells: 7 Number of Lateral Wells: 3
Tank Description

Type: Single Shell

Constructed: 1955

In-service: 06/30/59

Out of Service: $04 / 75$

Diameter: $7^{\circ}$

Usable Depth: 30.3'

Capacity: $1,000 \mathrm{~K}$ gallons

Bottom shape: Flat

Hanford Coordinates:

$41,307^{\prime}$ North

47,804' West

Air Lift Circulators: Yes (4)

Ventllation: Operating Exhauster

\section{Leak Detection System}

Surface Level: FIC Riser- None

Manual Tape Riser- R-11

LOW Riser(s)- None

\footnotetext{
Tank Status

Contents

Type: Non-Complexed Waste

Total Waste: $28 \mathrm{~K}$ gallons

Supernate Volume: OK gallons

Drainable Interstitial Liquid: OK gallons

Isolation Status

Date Interim Stabilized: $09 / 78$

Date Interim Isolated: $12 / 15 / 82$

Surface Level/Leak Status

Integrity Category: Assumed Leaker

Date Declared Confirmed or Assumed Leaker: 1975

Leak Volume Estimate: 2,500 Gallons (1983)

Manual Tape Surface Level: 11.00 Inches (11/04/92)

Last Photographed: 06/25/86

Photo Interpretation Comments:

Temperature Status

Photos reveal a dry, cracked surface of sludge with no visible liquid.

Highest temperature during 1992: $198.7^{\circ} \mathrm{F}$ (11/30/92)

Drywell Status

Comments: Temperatures are stable.

Comments: Current drywell profiles were stable and consistent with established baseline profiles.
} 


\section{WHC-EP-0625}

This page intentionally left blank. 


\section{Tank 241-A-105}

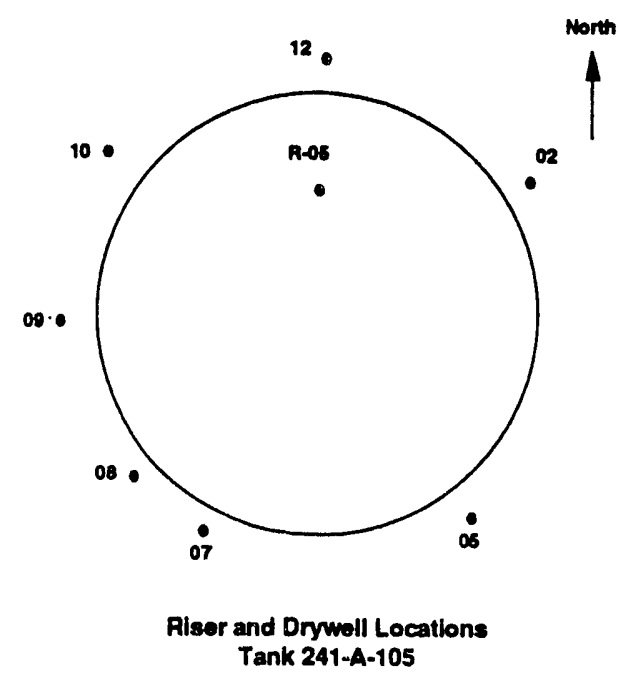

Number of External Drywells: 7

Number of Lateral Wells: 3

\section{Tank Status}

Contents

Type: Non-Complexed Waste

Total Waste: $19 \mathrm{~K}$ gallons

Supernate Volume: OK gallons

Drainable Interstitial Liquid: $4 \mathrm{~K}$ gallons

Isolation Status

Date Interim Stabllized: 07/79

Date Interim Isolated: 10/03/85

Surface Level/Leak Status

Integrity Category: Assumed Leaker

Date Declared Confirmed or Assumed Leaker: 1963

Leak Volume Estimate: 10,000 to 277.000 Gallons (1991)

Manual Tape Surface Level: 14.75 Inches (11/30/92)

Last Photographed: 08/20/86

Photo Interpretation Comments:

Photos reveal bare metal, damage to steel liner from bulge, and dry, cracked sludge, primarily around the tank perimeter. There is no visible liquid, and measurement anomalies should be expected as discarded tapes are directly beneath the manual tape.

Temperature Status

Highest temperature during 1992: $156^{\circ} \mathrm{F}(08 / 13 / 92)$

Comments: Temperatures are stable.

Drywell Status

Comments: Current drywell profiles were stable and consistent with established baseline profiles. 
WHC-EP-0625

This page intentionally left blank. 


\section{Tank 241-A-106}

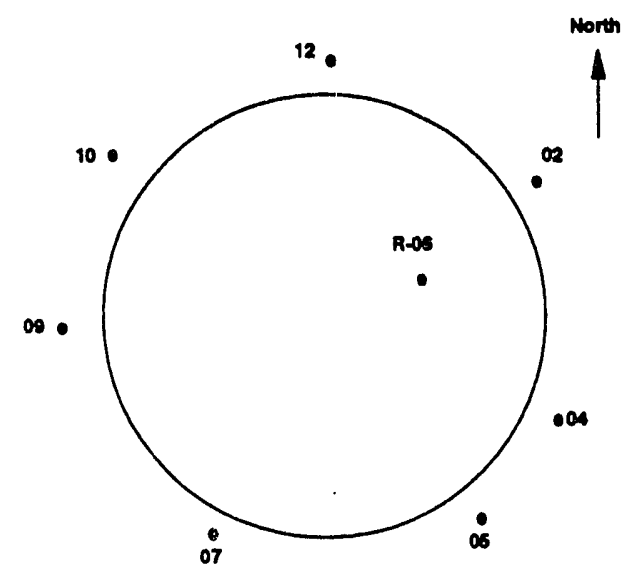

Fiser and Drywoll Locations Tank 241-A-106
Tank Description

Type: Single Shell

Constructed: 1955

In-service: 1957

Out of Service: 1980

Diameter: 75'

Usable Depth: 30.3'

Capacity: $1,000 \mathrm{~K}$ gallons

Bottom shape: Flat

Hanford Coordinates:

$41,307^{\prime}$ North

47,600' West

Air Lift Circulators: Yes (4)

Ventilation: Operating Exhauster

\section{Leak Detection System}

Surface Level: FIC Riser- R-06

Manual Tape Riser- None

LOW Riser(s)- None

Number of External Drywells: 7

Number of Lateral Weils: 3

\section{Tank Status}

Contents

Type: Double Shell Slurry Feed

Total Waste: $125 \mathrm{~K}$ gallons

Supernate Volume: OK gallons

Drainable Interstitial Liquid: $7 \mathrm{~K}$ gallons

Isolation Status

Date Interim Stabilized: $08 / 82$

Date Interim Isolated: $12 / 15 / 82$

Surface Level/Leak Status

Integrity Category: Sound

FIC Surface Level: Intrusion level set at 46.50 Inches (12/21/92)

Surface Level Comments: Intrusion Mode

Last Photographed: 08/17/86

Photo Interpretation Comments:

Photos reveal an uneven surface of sludge and a few sniall, isolated pockets of

Temperature Status what appear to be damp solids or very small puddles of liquid.

Highest temperature during 1992: $140^{\circ} \mathrm{F}(11 / 05 / 92)$

Drywell Status

Comments: Temperatures are stable.

Comments: Current drywell profiles were stable and consistent with established baseline proflles. 
WHC-EP-0625

This page intentionally left blank. 





WHC-EP-0625

This page intentionally left blank. 


\section{Tank 241-AN-101}

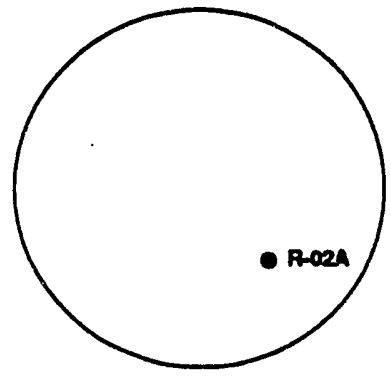

Riser and Drywell Locations Tank 241-AN-101

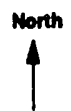

Number of External Drywells: None Number of Lateral Wells: None

\section{Tank Status}

\section{Contents}

Type: Dillute Non-Complexed Waste

Total Waste: $638 \mathrm{~K}$ gallons

Supernate Volume: $638 \mathrm{~K}$ gallons

Drainable Interstitial Liquid: OK gallons

Surface Level/Leak Status

Integrity Category: Sound

FIC Surface Level: 232.00 Inches (12/29/92)

Temperature Status

Highest temperature during 1992: $83^{\circ} \mathrm{F}(08 / 17 / 92)$

Comments: Temperatures are stable.
Tank Description

Type: Double Shell

Constructed: 1981

In-service: $09 / 81$

Diameter: $75^{\circ}$

Usable Depth: 35.2'

Capacity: $1,160.5 \mathrm{~K}$ gallons

Bottom shape: Flat

Hanford Coordinates:

$42,339.5^{\prime}$ North

47.506' West

Ventilation: Operating Exhauster

\section{Leak Detection System}

Surface Level:

FIC Riser- R-02A

Manual Tape Riser- None

LOW Riser(s)- None 
WHC-EP-0625

This page intentionally left blank. 


\section{Tank 241-AN-102}

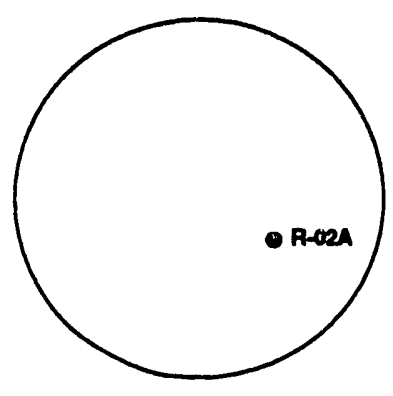

Riser and Drywell Locations Tank 241-AN- 102
Tank Description

Type: Double Shell

Constructed: 1981

In-service: $09 / 81$

Diameter: $\mathbf{7 5}^{\prime}$

Usable Depth: 35.2'

Capactty: $1,160.5 \mathrm{~K}$ gallons

Bottom shape: Flat

Hanford Coordinates:

42,339.5' North

47.613' West

Ventilation: Operating Exhauster

\section{Leak Detection System}

Surface Level:

FIC Riser- R-02A

Manual Tape Riser- None

Low Riser(s)- None

Number of External Drywells: None Number of Lateral Wells: None

\section{Tank Status}

\section{Contents}

Type: Complexant Concentrate Waste

Total Waste: $1,107 \mathrm{~K}$ gallons

Supernate Volume: 1,018K gallons

Drainable Interstitial Liquid: $3 \mathrm{~K}$ gallons

Surface Level/Leak Status

Integrity Category: Sound

FIC Surface Level: 401.80 Inches (12/29/92)

Temperature Status

Highest temperature during 1992: $100{ }^{\circ} \mathrm{F}(09 / 28 / 92)$

Comments: Temperatures are stable. 
WHC-EP-0625

This page intentionally left blank. 


\section{Tank 241-AN-103}

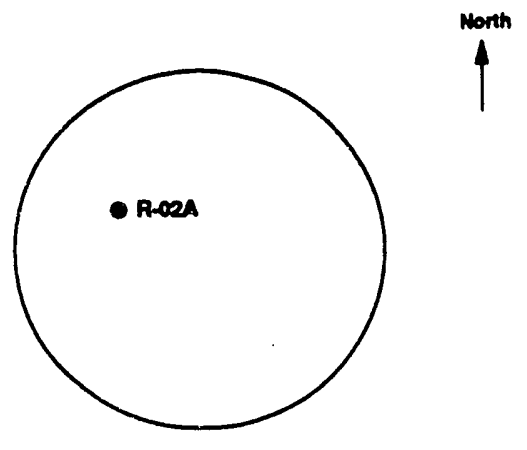

Riser and Drywell Locations Tank 241-AN-103

Number of External Drywells: None Number of Lateral Wells: None

\section{Tank Status}

Watch List: Hydrogen

Contents

Type: Double Shell Slurry

Total Waste: $953 \mathrm{~K}$ gallons

Supernate Volume: $16 \mathrm{~K}$ gallons

Drainable Interstitial Liquid: OK gallons

Surface Level/Leak Status

Integrity Category: Sound

FIC Surface Level: 346.80 Inches (12/29/92)

Last Photographed: 10/29/87

Photo Interpretation Comments: See attached photo.

Temperature Status

Highest temperature during 1992: $115^{\circ} \mathrm{F}(09 / 28 / 92)$

Comments: Temperatures are stable. 
WHC-EP-0625

This page intentionally left blank. 


\section{.}
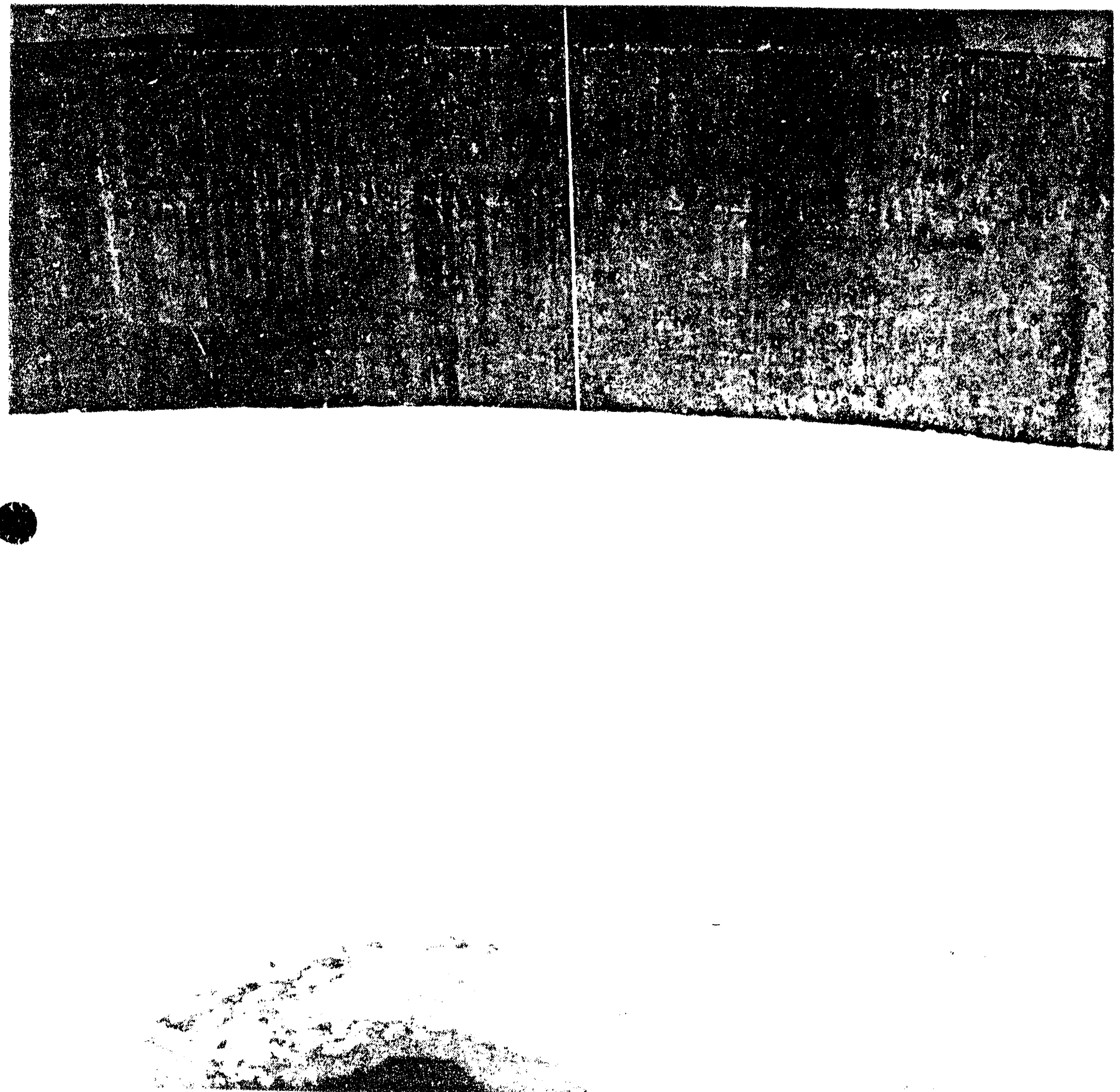
WHC-EP-0625

This page intentionally left blank. 


\section{Tank 241-AN-104}

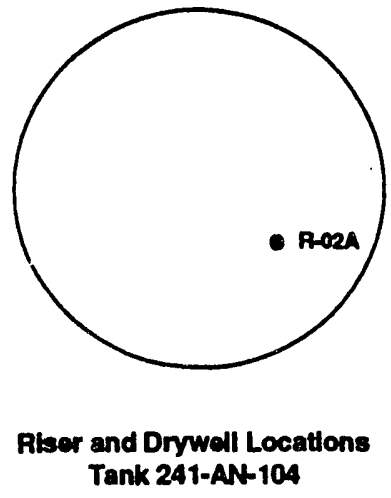

North

Number of External Drywells: None Number of Lateral Wells: None

\section{Tank Status}

Watch List: Hydrogen

Contents

Type: Double Shell Slurry Feed

Total Waste: 1,059K gallons

Supernate Volume: $795 \mathrm{~K}$ gallons

Drainable Interstitial Liquid: $25 \mathrm{~K}$ gallons

Surface Level/Leak Status

Integrity Category: Sound

FIC Surface Level: 388.70 Inches (12/29/92)

Last Photographed: 08/19/88

Photo Interpretation Comments: See attached photo.

Temperature Status

Highest temperature during 1992: $118^{\circ} \mathrm{F}(09 / 28 / 92)$

Comments: Temperatures are stable. 
WHC-EP-0625

This page intentionally left blank. 


\section{Tank: 241-AN-104}

Auqust 1988

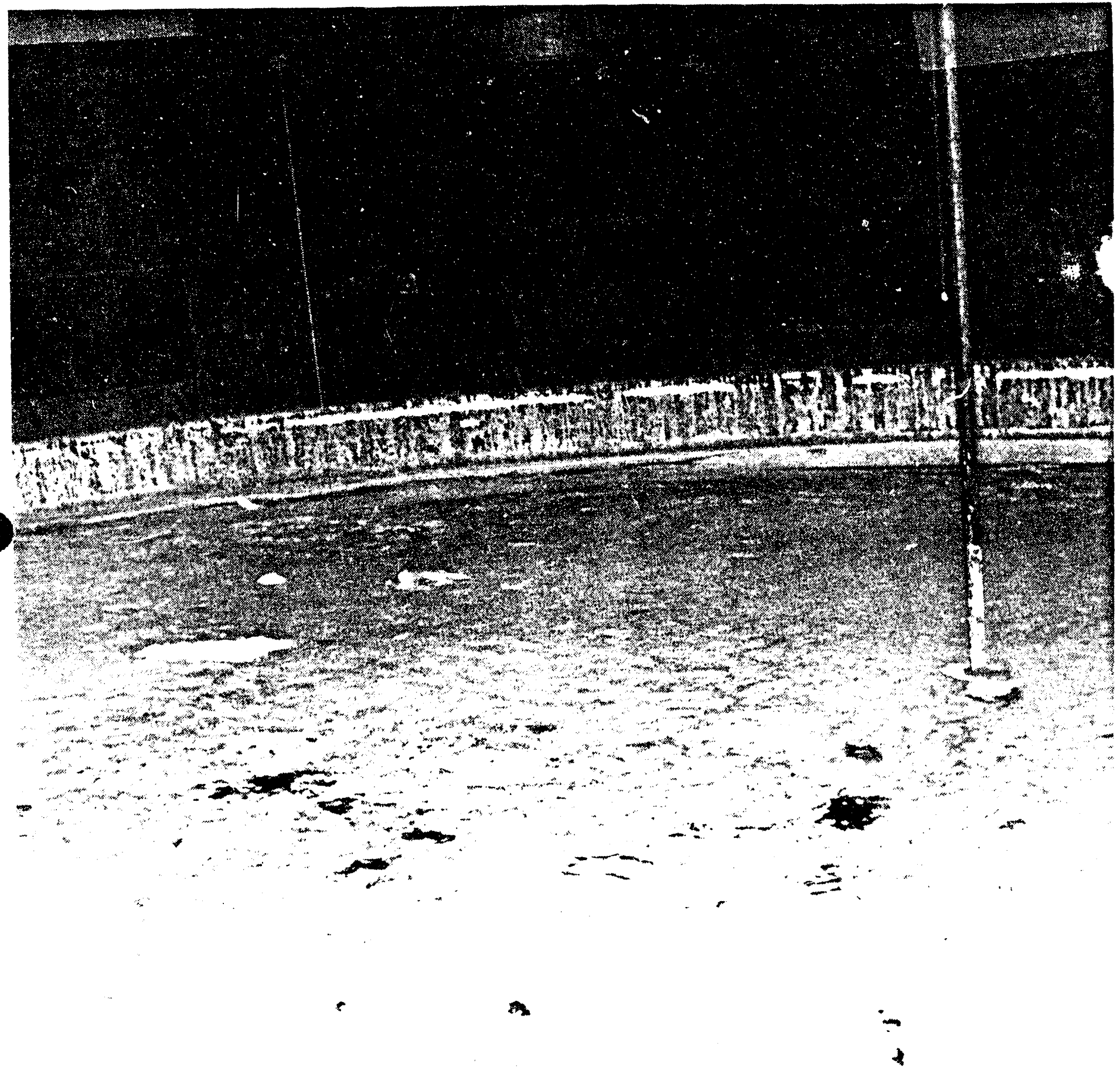




\section{WHC-EP-0625}

This page intentionaliy left blank. 


\section{Tank 241-AN-105}

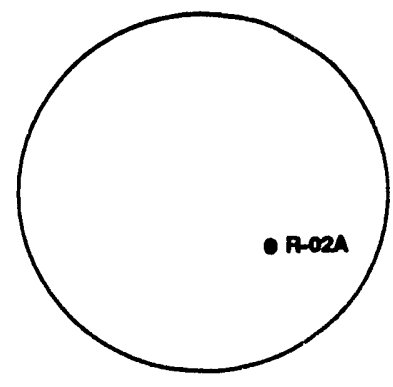

Riser and Drywell Locations Tank 241-AN-105
Tank Description

Type: Double Shell

Constructed: 1981

In-service: $09 / 81$

Diameter: 75 '

Usable Depth: 35.2'

Capacity: 1,160.5K gallons

Bottom shape: Flat

Hanford Coordinates: $42,446.5^{\prime}$ North 47,613' West

Ventilation: Operating Exhauster

\section{Leak Detection System}

Surface Level:

FIC Riser- R-02A

Manual Tape Riser- None LOW Riser(s)- None

Number of External Drywells: None

Number of Lateral Wells: None

\section{Tank Status}

\section{Watch List: Hydrogen}

\section{Contents}

Type: Double Shell Slurry Feed

Total Waste: $1,126 \mathrm{~K}$ gallons

Supernate Volume: $1,126 \mathrm{~K}$ gallons

Drainable Interstitial Liquid: OK gallons

Surface Level/Leak Status

Integrity Category: Sound

FIC Surface Level: 411.50 Inches (12/29/92)

Last Photographed: $01 / 26 / 88$

Photo Interpretation Comments: See attached photo.

Temperature Status

Highest temperature during 1992: $110^{\circ} \mathrm{F}(06 / 29 / 92)$

Comments: Temperatures are stable. 
WHC-EP-0625

This page intentionally left blank. 


\title{
Tank: 241-AN-105
}

\author{
Jimuary 1488
}

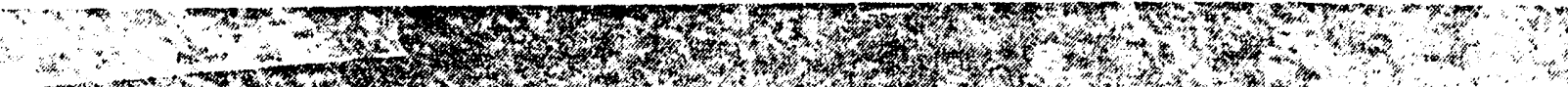
r

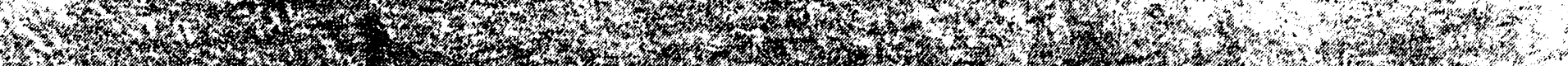

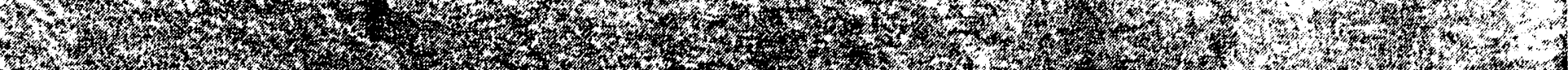

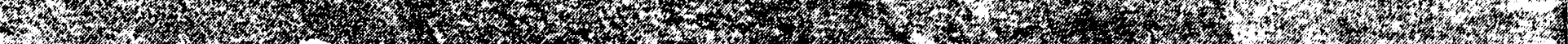
H

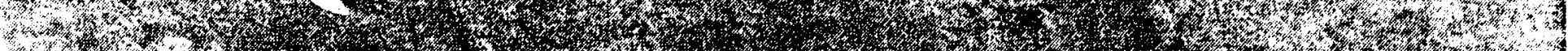

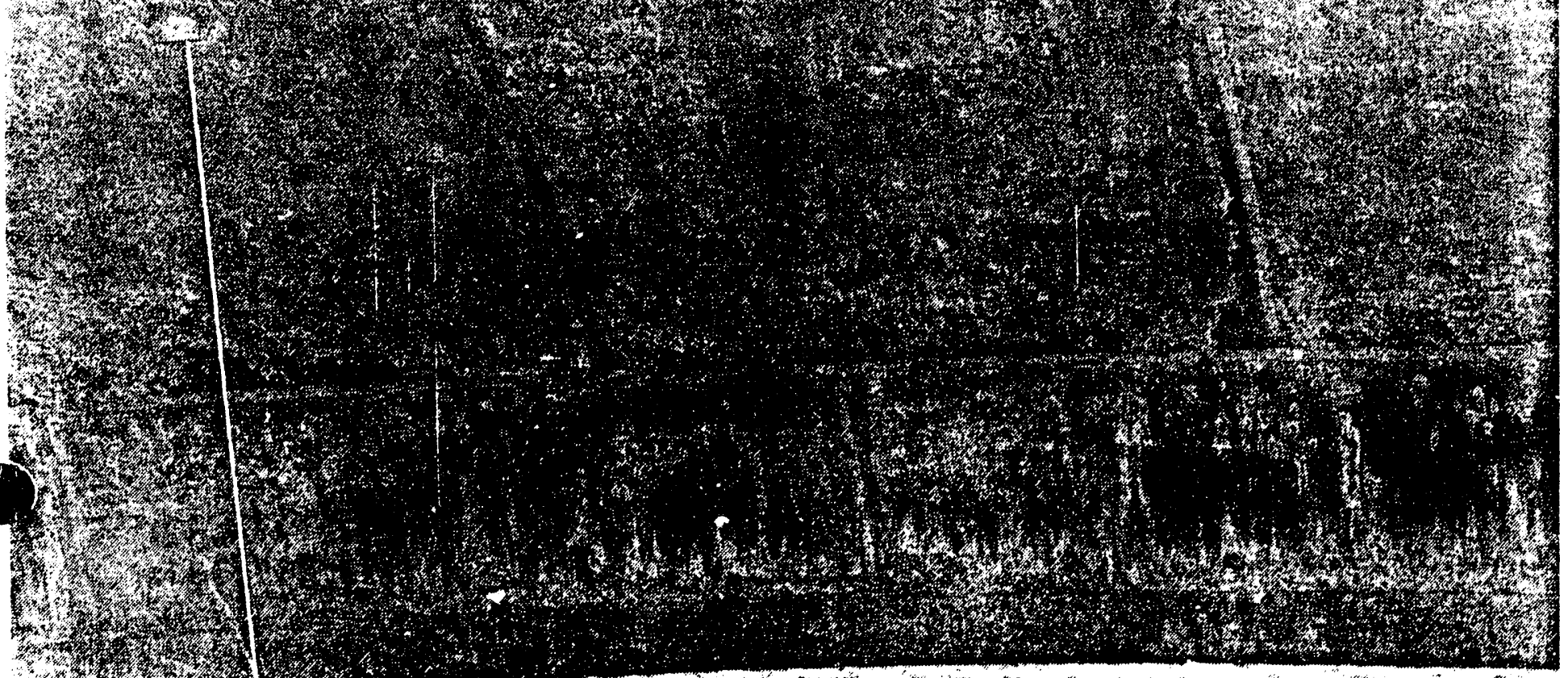

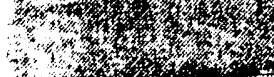

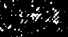

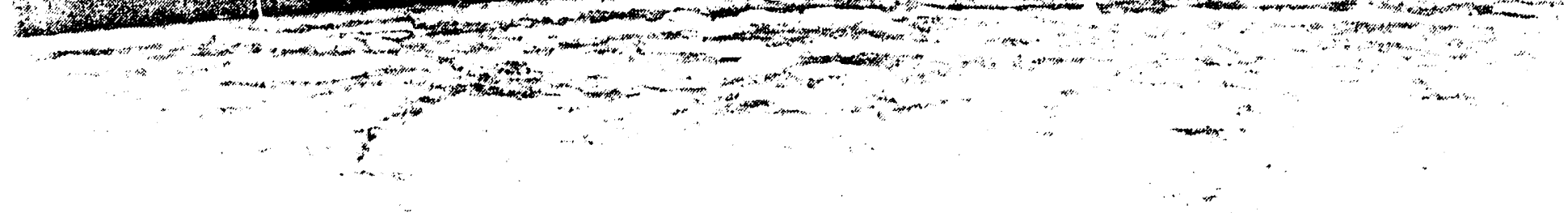


WHC-EP-0625

This page intentionally left blank. 


\section{Tank 241-AN-106}

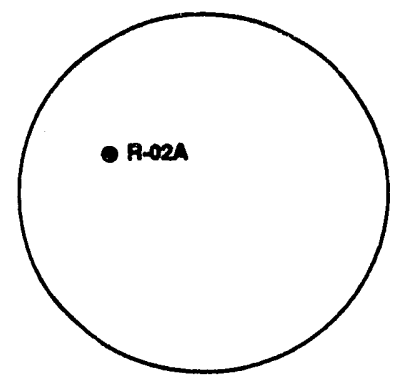

Riser and Drywell Locations Tank 241-AN-106
Tank Description

Type: Double Shell

Constructed: 1981

In-service: $09 / 81$

Diameter: $\mathbf{7 5}^{\prime}$

Usable Depth: 35.2'

Capacity: $1,160.5 \mathrm{~K}$ gallons

Bottom shape: Flat

Hanford Coordinates: 42.446.5' North 47.720' West

Ventilation: Operating Exhauster

\section{Leak Detection System}

Surface Level:

FIC Riser- R-02A

Manual Tape Riser- None LOW Riser(s)- None

Number of External Drywells: None

Number of Lateral Wells: None

\section{Tank Status}

\section{Contents}

Type: Concentrated Phosphate Waste

Total Waste: $1,013 \mathrm{~K}$ gallons

Supernate Volume: $996 \mathrm{~K}$ gallons

Drainable Interstitial Liquid: OK gallons

\section{Surface Level/Leak Status}

Integrity Category: Sound

FIC Surface Level: 8.50 Inches (12/29/92)

Temperature Status

Highest temperature during 1992: $80^{\circ} \mathrm{F}(09 / 28 / 92)$

Comments: Temperatures are stable. 
WHC-EP-0625

This page intentionally left blank. 


\section{Tank 241-AN-107}

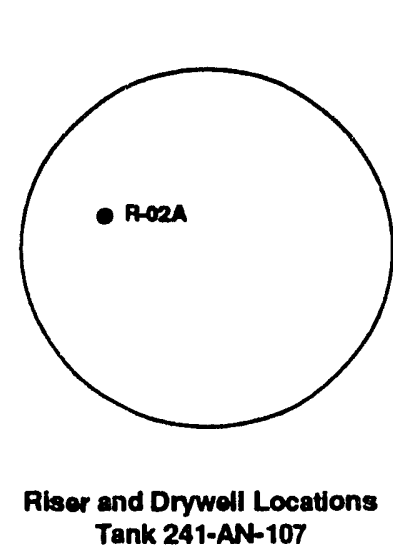

4

Number of External Drywells: None

Number of Lateral Wells: None

\section{Tank Status}

\section{Contents}

Type: Complexant Concentrate Waste

Total Waste: $1,071 \mathrm{~K}$ gallons

Supernate Volume: $937 \mathrm{~K}$ gallons

Drainable Interstitial Liquid: 9K gallons

Surface Level/Leak Status

Integrity Category: Sound

FIC Surface Level: 388.80 Inches (12/29/92)

Last Photographed: 09/01/88

Temperature Status

Highest temperature during 1992: $100{ }^{\circ} \mathrm{F}(09 / 28 / 92)$

Comments: Temperatures are stable.

\section{Tank Description}

Type: Double Shell

Constructed: 1981

In-service: 09/81

Diameter: $75^{\prime}$

Usable Depth: 35.2'

Capacity: $1,160.5 \mathrm{~K}$ gallons

Bottom shape: Flat

Hanford Coordinates:

42,553.5' North

47.720' West

Ventilation: Operating Exhauster

\section{Leak Detection System}

Surface Level:

FIC Riser- R-02A

Manual Tape Riser- None

LOW Riser(s)- None 
WHC-EP-0625

This page intentionally left blank. 
WHC-EP-0625

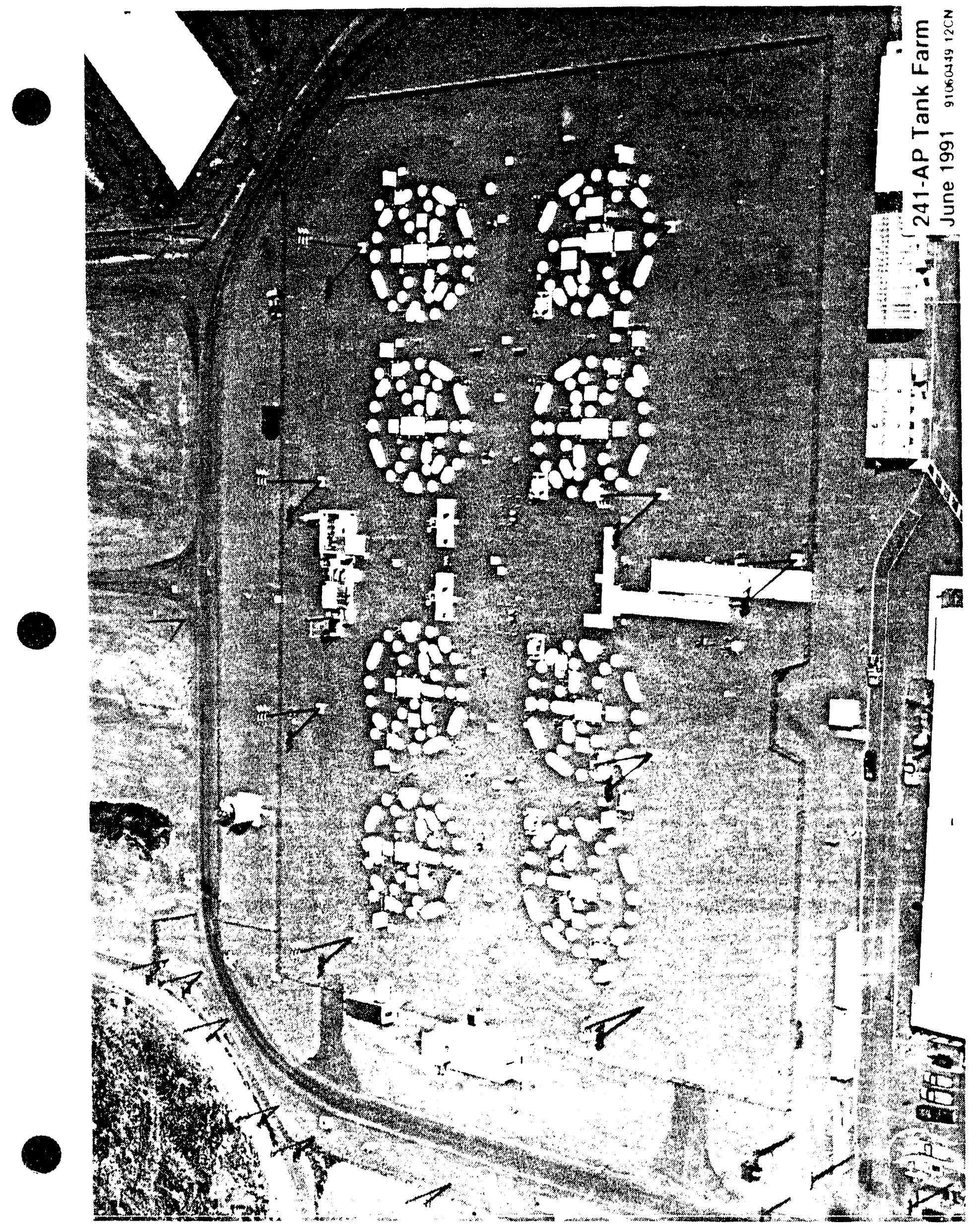


WHC-EP-0625

This page intentionally left blank. 


\section{Tank 241-AP-101}

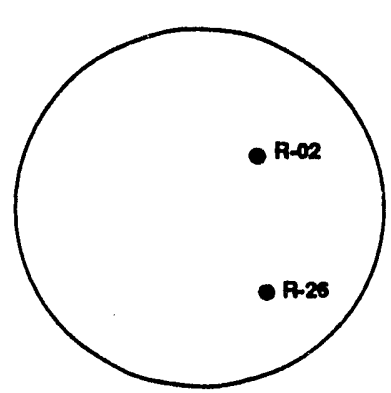

Riser and Drywell Locations Tank 241-AP-101
4

Number of External Drywells: None

Number of Lateral Wells: None

\section{Tank Status}

Contents

Type: Dilute Non-Complexed Waste

Total Waste: $1,062 \mathrm{~K}$ gallons

Supernate Volume: $1,062 \mathrm{~K}$ gallons

Drainable Interstitial Liquid: OK gallons

\section{Surface Level/Leak Status}

Integrity Category: Sound

Manual Tape Surface Level: 385.75 Inches (12/29/92)

FIC Surface Level: 385.90 Inches (12/29/92)

Temperature Status

Highest temperature during 1992: $73^{\circ} \mathrm{F}(09 / 28 / 92)$

Comments: Temperatures are stable.
Tank Description

Type: Double Shell

Constructed: 1986

In-service: 1986

Diameter: $7^{\circ}$

Usable Depth: $35^{\prime}$

Capacity: $1.160 \mathrm{~K}$ gallons

Bottom shape: Flat

Hanford Coordinates:

40.666' North

47,100' West

Ventilation: Operating Exhauster

\section{Leak Detection System}

Surface Level:

FIC Riser- R-02

Manual Tape Riser- R-26

LOW Riser(s)- None 
WHC-EP-0625

This page intentionally left blank. 
WHC-EP-0625

\section{Tank 241-AP-102}

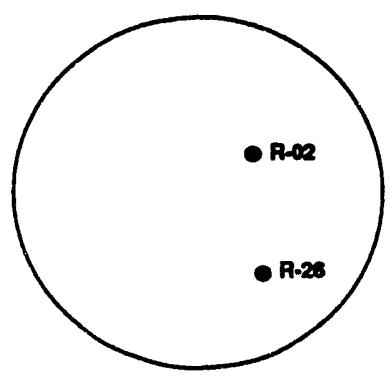

Riser and Drywell Locettons Tank 241-AP-102

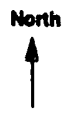

Number of External Drywells: None Number of Lateral Wells: None

\section{Tank Status}

Contents

Type: Dilute Non-Complexed Waste

Total Waste: $137 \mathrm{~K}$ gallons

Supernate Volume: $137 \mathrm{~K}$ gallons

Drainable Interstitial Liquid: OK gallons

Surface Level/Leak Status

Integrity Category: Sound

Manual Tape Surface Level: 402.25 Inches (12/29/92)

FIC Surface Level: 401.90 Inches (12/29/92)

Temperature Status

Highest temperature during 1992: $77^{\circ} \mathrm{F}(12 / 28 / 92)$

Comments: Temperatures are stable.
Tank Description

Type: Double Shell

Constructed: 1986

In-service: 1986

Diameter: $7^{\circ}$

Usable Depth: $3^{\circ}$

Capacity: 1,160K gallons

Bottom shape: Flat

Hanford Coordinates:

$40,666^{\circ}$ North

46,968' West

Ventilation: Operating Exhauster

\section{Leak Detection System}

Surface Level:

FIC Riser- R-02

Manual Tape Riser- R-26

LOW Riser(s)- None 
WHC-EP-0625

This page intentionally left blank. 


\section{Tank 241-AP-103}

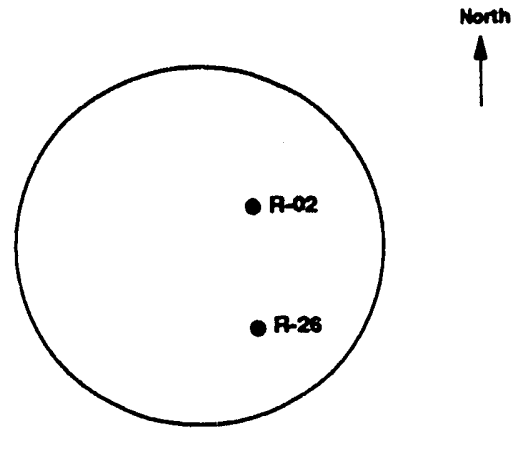

Riser and Drywell Locations Tank 241-AP-103
Tank Description Type: Double Shell Constructed: 1986 In-service: 1986 Diameter: $75^{\circ}$ Usable Depth: $35^{\circ}$ Capactty: $1,160 \mathrm{~K}$ gallons Bottom shape: Flat Hanford Coordinates: $40,559^{\prime}$ North 47,100, West Ventilation: Operating Exhauster

\section{Leak Detection System}

Surface Level: FIC Riser- R-02 Manual Tape Riser- R-26 LOW Riser(s)- None

Number of External Drywells: None Number of Lateral Wells: None

\section{Tank Status}

Contents

Type: Dilute Non-Complexed Waste

Total Waste: $1.134 \mathrm{~K}$ gallons

Supernate Volume: $1.134 \mathrm{~K}$ gallons

Drainable Interstitial Liquid: OK gallons

\section{Surface Level/Leak Status}

Integrity Category: Sound

Manual Tape Surface Level: 412.00 Inches (12/29/92)

Temperature Status

Highest temperature during 1992: $65^{\circ} \mathrm{F}(11 / 09 / 92)$

Comments: Temperatures are stable. 
WHC-EP-0625

This page intentionally left blank. 


\section{Tank 241-AP-104}

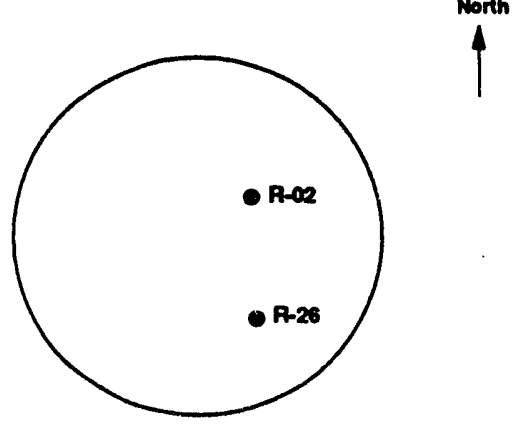

Riser and Drywell Locations Tank 241-AP-104
Tank Description Type: Double Sheli Constructed: 1986 In-service: 1986 Diameter: $75^{\circ}$ Usable Depth: $35^{\circ}$ Capactty: $1,160 \mathrm{~K}$ gallons Bottom shape: Flat Hanford Coordinates: 40,559' North $46,968^{\prime}$ 'West

Ventulation: Operating Exhauster

\section{Leak Detection System}

Surface Level:

FIC Riser- R-02

Manual Tape Riser- R-26 LOW Riser(s)- None

Number of External Drywells: None

Number of Lateral Wells: None

\section{Tank Status}

Contents

Type: Dilute Non-Complexed Waste

Total Waste: $20 \mathrm{~K}$ gallons

Supernate Volume: $20 \mathrm{~K}$ gallons

Drainable Interstitial Liquid: OK gallons

Surface Level/Leak Status

Integrity Category: Sound

FIC Surface Level: 7.10 Inches (12/29/92)

Temperature Status

Highest temperature during 1992: $92^{\circ} \mathrm{F}(12 / 14 / 92)$

Comments: Temperatures are stable. 
WHC-EP-0625

This page intentionally left blank. 


\section{Tank 241-AP-105}

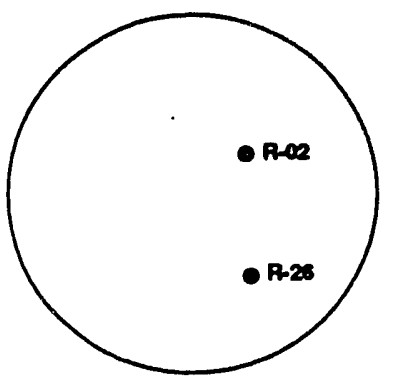

Rber and Drywell Locations Tank 241-AP-105

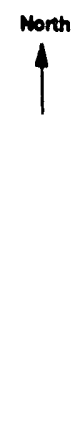

Number of External Drywells: None Number of Lateral Wells: None

\section{Tank Status}

Contents

Type: Double Shell Slurry Feed

Total Waste: $824 \mathrm{~K}$ gallons

Supernate Volume: $824 \mathrm{~K}$ gallons

Drainable Interstitial Liquid: $\mathrm{OK}$ gallons

Surface Level/Leak Status

Integrity Category: Sound

Manual Tape Surface Level: 298.50 (12/29/92)

FIC Surface Level: 299.20 Inches (12/29/92)

Temperature Status

Highest temperature during 1992: $87^{\circ} \mathrm{F}(08 / 23 / 92)$

Comments: Temperatures are stable.
Tank Description

Type: Double Shell

Constructed: 1986

In-service: 1986

Diameter: $75^{\circ}$

Usable Depth: $35^{\circ}$

Capactty: $1,160 \mathrm{~K}$ gallons

Bottom shape: Flat

Hanford Coordinates: 40,393' North 47,100' West

Ventilation: Operating Exhauster

\section{Leak Detection System}

Surface Level:

FIC Riser- R-02

Manual Tape Riser- R-26

LOW Riser(s)- None 
WHC-EP-0625

This page intentionally left blank. 
WHC-EP-0625

\section{Tank 241-AP-106}

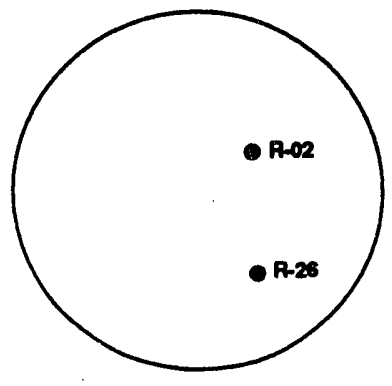

Riser and Drywell Locations Tank 241-AP-106
4

Number of External Drywells: None

Number of Lateral Wells: None

\section{Tank Status}

Contents

Type: Dilute Non-Complexed Waste

Total Waste: $1,131 \mathrm{~K}$ gallons

Supernate Volume: $1,131 \mathrm{~K}$ gallons

Drainable Interstitial Liquid: OK gallons

Surface Level/Leak Status

Integrity Category: Sound

Manual Tape Surface Level: 411.00 Inches (12/29/92)

FIC Surface Level: 411.00 Inches (12/29/92)

Temperature Status

Highest temperature durin', 1992: $80^{\circ} \mathrm{F}(08 / 23 / 92)$

Comments: Temperatures are stable.
Tank Description

Type: Double Shell

Constructed: 1986

In-service: 1986

Diameter: $75^{\prime}$

Usable Depth: $35^{\prime}$

Capacity: $1,160 \mathrm{~K}$ gallons

Bottom shape: Flat

Hanford Coordinates:

$40,393^{\prime}$ North

46,968' West

Ventilation: Operating Exhauster

\section{Leak Detection System}

Surface Level:

FIC Riser- R-02

Manual Tape Riser- R-26

LOW Riser(s)- None 
WHC-EP-0625

This page intentionally left blank. 
WHC-EP-0625

\section{Tank 241-AP-107}

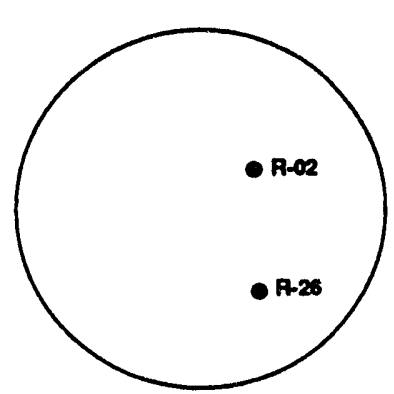

Riser and Drywell Locations Tank 241-AP-107
1 Number of Lateral Wells: None

\section{Tank Status}

Contents

Type: Dilute Non-Complexed Waste

Total Waste: $1,122 \mathrm{~K}$ gallons

Supernate Volume: $1,122 \mathrm{~K}$ gallons

Drainable Interstitial Liquid: OK gallons

\section{Surface Level/Leak Status}

Integrity Category: Sound

Manual Tape Surface Level: 405.25 Inches (12/29/92)

FIC Surface Level: 406.40 Inches (12/29/92)

Temperature Status

Highest temperature during 1992: $71^{\circ} \mathrm{F}(08 / 23 / 92)$

Comments: Temperatures are stable.
Tank Description

Type: Double Shell Constructed: 1986

In-service: 1986

Diameter: $7^{\circ}$

Usable Depth: $35^{\circ}$

Capacity: $1,160 \mathrm{~K}$ gallons

Bottom shape: Flat

Hanford Coordinates:

$40,286^{\prime}$ North

$47,100^{\prime}$ 'West

Ventilation: Operating Exhauster

\section{Leak Detection System}

Surface Level:

FIC Riser- R-02

Manual Tape Riser- R-26

LOW Riser(s)- None 
WHC-EP-0625

This page intentionally left blank. 
WHC-EP-0625

\section{Tank 241-AP-108}

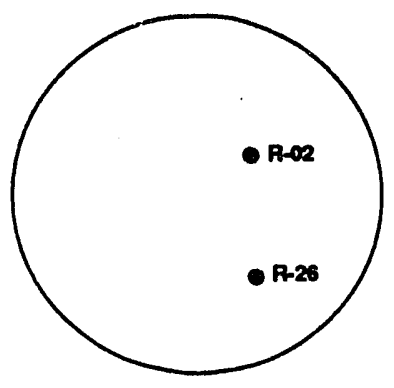

Riser and Drywell Locations Tank 241-AP-108
Tank Description Type: Double Shell Constructed: 1986 In-service: 1986 Diameter: 75' Usable Depth: 35' Capacity: $1,160 \mathrm{~K}$ gallons Bottom shape: Flat Hanford Coordinates: 40,286' North 46,968' West Ventilation: Operating Exhauster

\section{Leak Detection System}

Surface Level:

FIC Riser- R-02

Manual Tape Riser- R-26

LOW Riser(s)- None

Number of External Drywells: None Number of Lateral Wells: None

\section{Tank Status}

\section{Contents}

Type: Dilute Non-Complexed Waste Total Waste: 903K gallons

Supernate Volume: 903K gallons

Drainable Interstitial Liquid: OK gallons

Surface Level/Leak Status Integrity Category: Sound

Manual Tape Surface Level: 327.50 Inches (12/30/92)

FIC Surface Level: 327.30 Inches (12/30/92)

Temperature Status

Highest temperature during 1992: $69^{\circ} \mathrm{F}(11 / 02 / 92)$

romments: Temperatures are stable. 
WHC-EP-0625

This page intentionally left blank. 







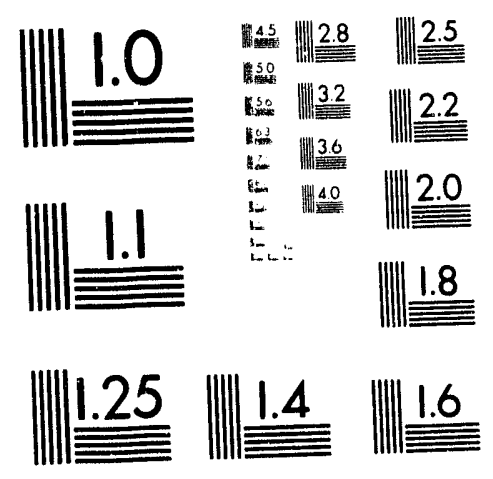



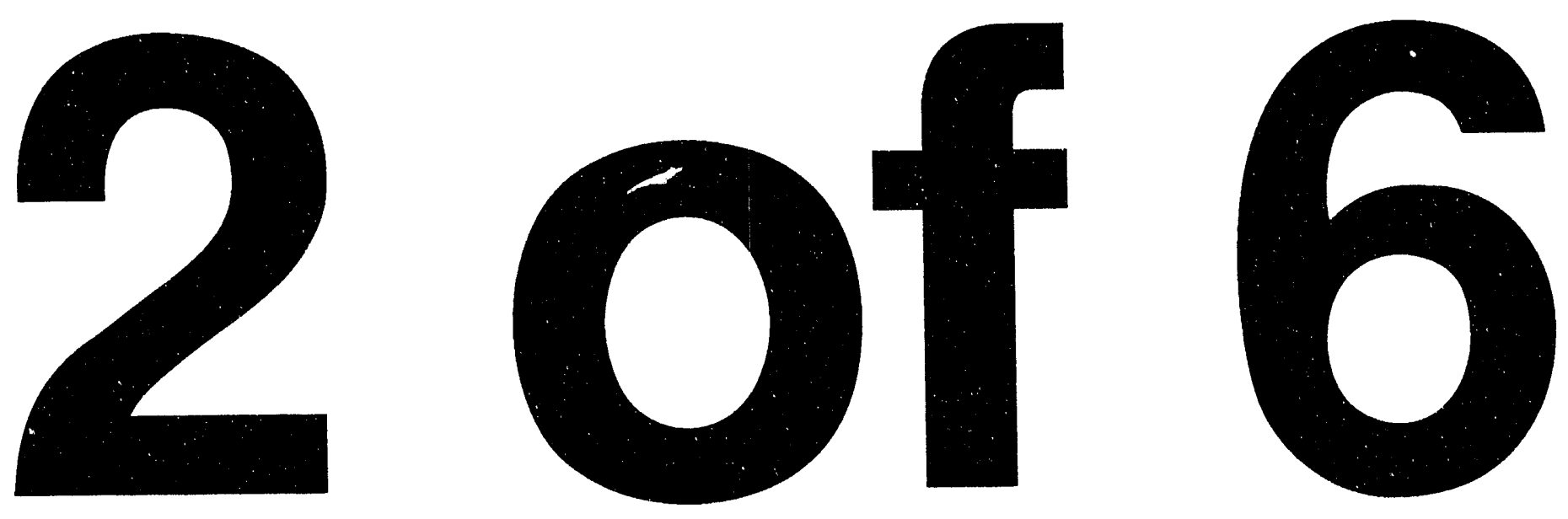
WHC-EP-0625

This page intentionally left blank. 


\section{Tank 241-AW-101}

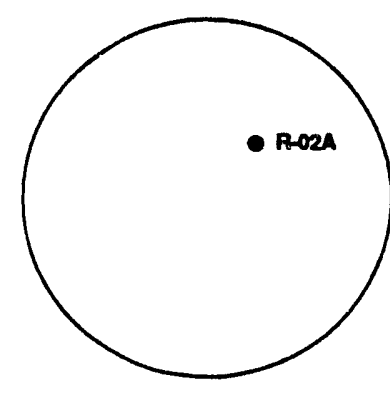

Riser and Drywell Locations Tank 241-AW-101
Tank Description

Type: Double Shell

Constructed: 1980

In-service: $07 / 31 / 80$

Diameter: $75^{\circ}$

Usable Depth: 35.2'

Capacity: $1.160 .5 \mathrm{~K}$ gallons

Bottom shape: Flat

Hanford Coordinates:

40.704' North

47,752' West

Ventilation: Operating Exhauster

\section{Leak Detection System}

Surface Level:

FIC Riser- R-02A

Manual Tape Riser- Yes

LOW Riser(s)- None

Number of External Drywells: None

Number of Lateral Wells: None

\section{Tank Status}

Contents

Type: Double Shell Slurry Feed

Total Waste: $1,126 \mathrm{~K}$ gallons

Supernate Volume: $1.042 \mathrm{~K}$ gallons

Drainable Interstitial Liquid: $2 \mathrm{~K}$ gallons

Surface Level/Leak Status

Integrity Category: Sound

Manual Tape Surface Level: 416.25 Inches (12/29/92)

FIC Surface Level: 408.50 Inches (12/29/92)

Last Photographed: 03/17/88

Temperature Status

Highest temperature during 1992: $100{ }^{\circ} \mathrm{F}(07 / 20 / 92)$

Comments: Temperatures are stable. 
WHC-EP-0625

This page intentionally left blank. 


\section{Tank: 241-AW-101}

March 1938

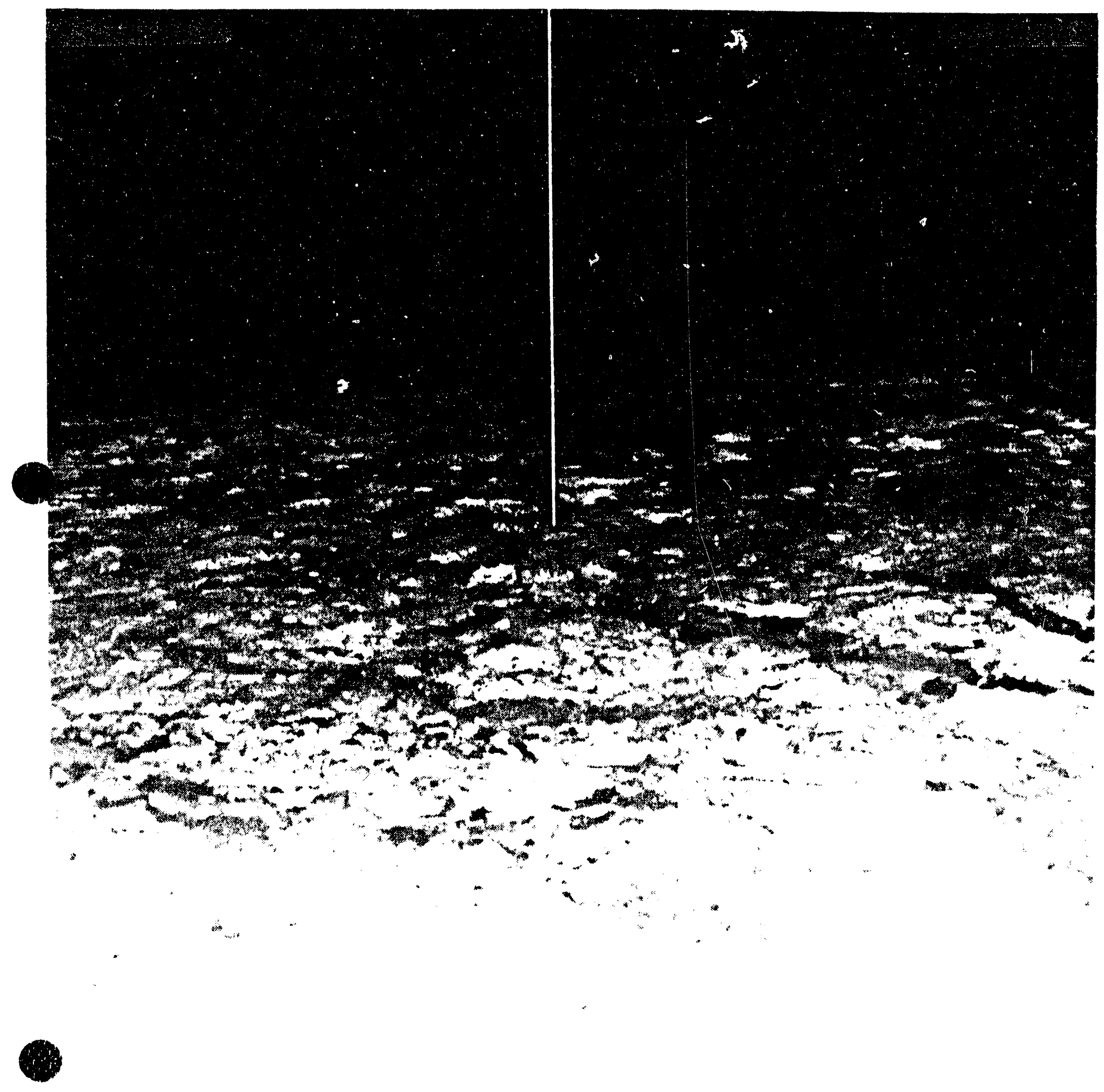


WHC-EP-0625

This page intentionally left blank. 


\section{Tank 241-AW-102}

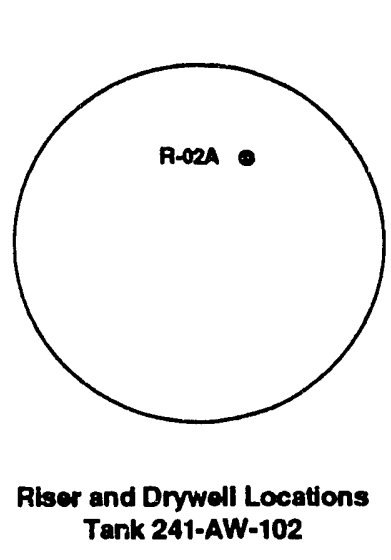

Number of External Drywells: None Number of Lateral Wells: None

\section{Tank Status}

Contents

Type: Dilute Non-Complexed Waste

Total Waste: $679 \mathrm{~K}$ gallons

Supernate Volume: $678 \mathrm{~K}$ gallons

Drainable Interstitial Liquid: OK gallons

Surface Level/Leak Status

Integrity Category: Sound

Manual Tape Surface Level: 272.50 Inches (12/29/92)

FIC Surface Level: 273.40 Inches (12/29/92)

Last Photographed: 02/02/83

Temperature Status

Highest temperature during 1992: $89^{\circ} \mathrm{F}(04 / 27 / 92)$

Comments: Temperatures are stable.
Tank Description Type: Double Shell Constructed: 1980 In-service: 1980 Diameter: $75^{\circ}$ Usable Depth: 35.2' Capacity: $1,160.5 \mathrm{~K}$ gallons Bottom shape: Flat Hanford Coordinates: $40,704^{\prime}$ North 47.645' West Ventilation: Operating Exhauster

\section{Leak Detection System} Surface Level:

FIC Riser- R-02A

Manual Tape Riser- Yes LOW Riser(s)- None 
WHC-EP-0625

This page intentionally left blank. 


\title{
Tank 241-AW-103
}

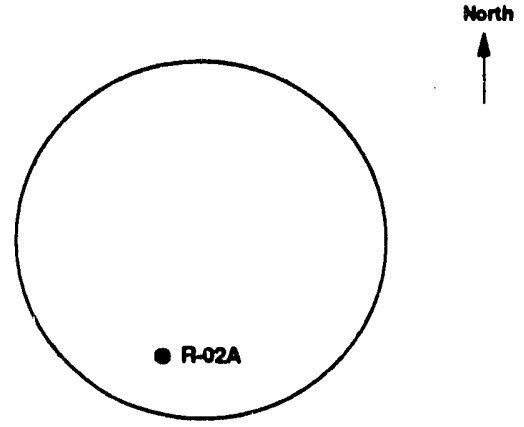

Riser and Drywell Locations Tank 241-AW-103
Tank Description

Type: Double Shell

Constructed: 1980

In-service: 1980

Diameter: $7^{\circ}$

Usable Depth: 35.2'

Capacity: $1,160.5 \mathrm{~K}$ gallons

Bottorn shape: Flat

Hanford Coordinates:

$40,597^{\circ}$ North

47.752' West

Ventilation: Operating Exhauster

\section{Leak Detection System}

Surface Level:

FIC Riser- R-02A

Manual Tape Riser- Yes

LOW Riser(s)- None

Number of External Drywells: None

Number of Lateral Wells: None

\author{
Tank Status \\ Contents \\ Type: PUREX Neutralized Cladding Removal Waste (TRU) \\ Total Waste: 646K gallons \\ Supernate Volume: $283 \mathrm{~K}$ gallons \\ Drainable Interstitial Liquid: $37 \mathrm{~K}$ gallons \\ Surface Level/Leak Status \\ Integrity Category: Sound \\ Manual Tape Surface Level: 234.50 Inches (12/29/92) \\ Temperature Status \\ Highest temperature during 1992: $88^{\circ} \mathrm{F}(04 / 27 / 92)$ \\ Comments: Temperatures are stable.
}


WHC-EP-0625

This page intentionally left blank. 


\section{Tank 241-AW-104}

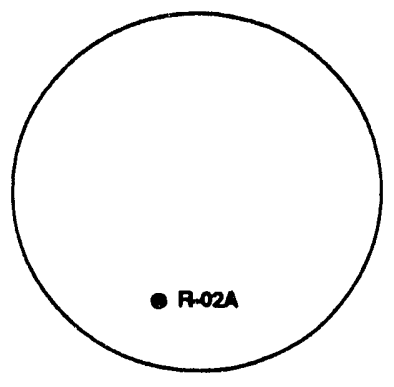

Riser and Drywell Locations Tank 241-AW-104
Tank Description

Type: Double Shell Constructed: 1980

In-service: 1980

Diameter: $7^{\circ}$

Usable Depth: 35.2'

Capacity: $1.160 .5 \mathrm{~K}$ gallons

Bottom shape: Flat

Hanford Coordinates:

$40,597^{\prime}$ North

$47,645^{\prime}$ West

Ventilation: Operating Exhauster

\section{Leak Detection System}

Surface Level:

FIC Riser- R-02A

Manual Tape Riser- Yes

LOW Riser(s)- None

Number of External Drywells: None

Number of Lateral Wells: None

\section{Tank Status}

Contents

Type: Dilute Non-Complexed Waste

Total Waste: $1,125 \mathrm{~K}$ gallons

Supernate Volume: .835K gallons

Drainable Interstitial Liquid: $49 \mathrm{~K}$ gallons

Surface Level/Leak Status

Integrity Category: Sound

Manual Tape Surface Level: 408.00 Inches (12/29/92)

FIC Surface Level: 408.70 Inches (12/29/92)

Last Photographed: 02/02/83

Temperature Status

Highest temperature during 1992: $86^{\circ} \mathrm{F}(01 / 06 / 92)$

Comments: Temperatures are stable. 
WHC-EP-0625

This page intentionally left blaik. 


\title{
Tank 241-AW-105
}

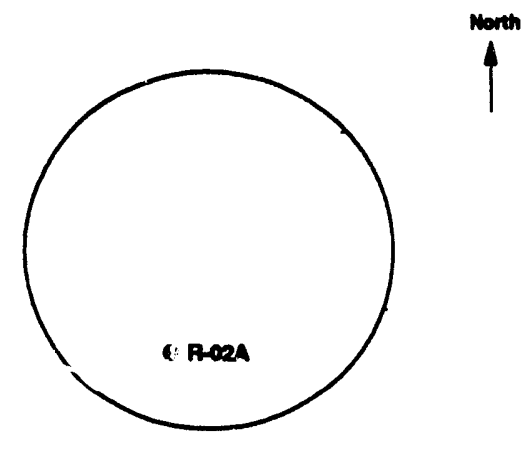

Rlece and Drywell Locations Tank 241-AW-10.
Tank Description Type: Double Shell Constructed: 1980

In-service: 1980

Diameter: $75^{\circ}$

Usable Depth: 35.2'

Capacity: 1,160.5K gallons

Bottom shape: Flat

Hanford Coordinates: $40,490^{\prime}$ North 47,752, West

Ventilation: Operating Exhauster

\section{Leak Detection System}

Surface Level:

FIC Riser- R-02A

Manual Tape Riser- Yes

LOW Riser(s)- None

Number of External Drywells: None

Number of Lateral Wells: None

\author{
Tank Status \\ Contents \\ Type: DN/PD \\ Total Waste: $911 \mathrm{~K}$ gallons \\ Supernate Volume: $614 \mathrm{~K}$ gallons \\ Drainable Interstitial Liquid: $29 \mathrm{~K}$ gallons \\ Surface Level/Leak Status \\ Integrity Category: Sound \\ Manual Tape Surface Level: 345.50 Inches (12/29/92) \\ FIC Surface Level: 346.50 Inches (12/29/92) \\ Temperature Status \\ Highest temperature during 1992: $85^{\circ} \mathrm{F}(05 / 25 / 92)$ \\ Comments: Temperatures are stable.
}


WHC-EP-0625

This page intentionally left blank. 


\section{Tank 241-AW-106}

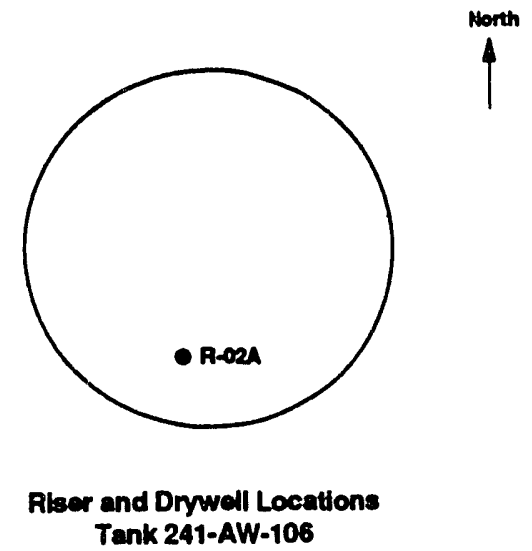

Number of External Drywells: None Number of Lateral Wells: None

\section{Tank Status}

Contents

Type: Dilute Non-Complexed Waste

Total Waste: $888 \mathrm{~K}$ gallons

Supernate Volume: $592 \mathrm{~K}$ gallons

Drainable Interstitial Liquid: $42 \mathrm{~K}$ gallons

\section{Surface Level/Leak Status}

Integrity Category: Sound

Manual Tape Surface Level: 347.00 Inches (12/29/92)

FIC Surface Level: 347.90 Inches (12/29/92)

Last Photographed: 02/02/83

Temperature Status

Highest temperature during 1992: $92{ }^{\circ} \mathrm{F}(03 / 23 / 92)$

Comments: Temperatures are stable.
Tank Description

Type: Double Shell

Constructed: 1980

In-service: 1980

Diameter: $7^{\circ}$

Usable Depth: 35.2'

Capacity: $1,160.5 \mathrm{~K}$ gallons

Bottom shape: Flat

Hanford Coordinates: 40,490 ' North 47,645' West

Ventilation: Operating Exhauster

\section{Leak Detection System}

Surface Level:

FIC Riser- R-02A

Manual Tape Riser- Yes

LOW Riser(s)- None 


\section{WHC-EP-0625}

This page intentionally left blank. 


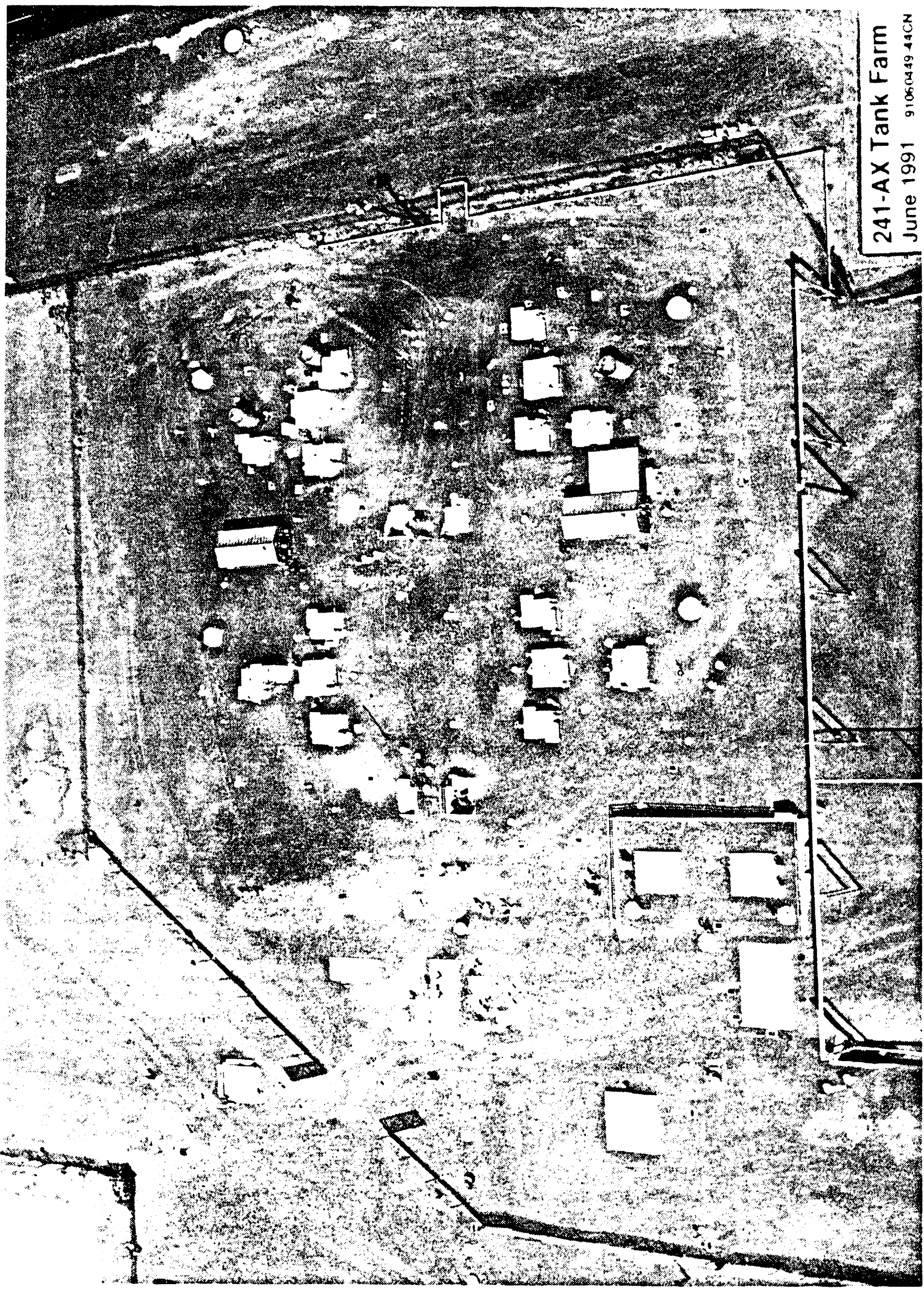




\section{WHC-EP-0625}

This page intentionally left blank. 


\section{Tank 241-AX-101}

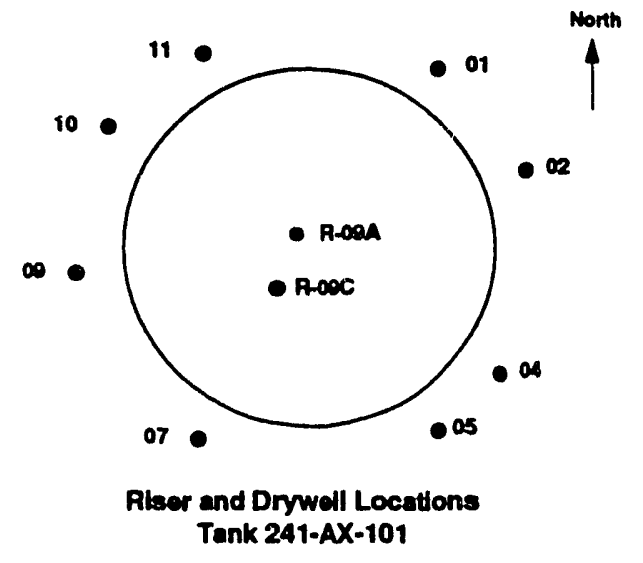

Number of External Drywells: 8

Number of Lateral Wells: None

\section{Tank Status}

Watch List: Hydrogen

Contents

Type: Double Shell Slurry Feed

Total Waste: $748 \mathrm{~K}$ gallons

Supernate Volume: OK gallons

Drainable Interstitial Liquid: $320 \mathrm{~K}$ gallons

Isolation Status

Date Partially Interim Isolated: $12 / 15 / 82$

\section{Surface Level/Leak Status}

Integrity Category: Sound

FIC Surface Level: Intrusion level set at 278.20 Inches (12/28/92)

Surface Level Comments: Intrusion Mode

Last Photographed: 08/18/87

Photo Interpretation Comments: See attached photo.

Temperature Status

Highest temperature during 1992: $141{ }^{\circ} \mathrm{F}(01 / 24 / 92)$

Comments: Temperatures are stable.

\section{Drywell Status}

Comments: Current drywell profiles were stable and consistent with established basellne proflles.
Tank Description

Type: Single Shell

Constructed: 1964

In-service: 1965

Out of Service: $11 / 12 / 80$

Diameter: $75^{\prime}$

Usable Depth: 30.3'

Capacity: $1,000 \mathrm{~K}$ gallons

Bottom shape: Flat

Hanford Coordinates:

$41,731^{\circ}$ North

47,475' West

Ventllation: Passive

\section{Leak Detection System}

Surface Level:

FIC Riser- R-09C

Manual Tape Riser- None

LOW Riser(s)- R-09A 
WHC-EP-0625

This page intentionally left blank. 


\section{Tank: 241-AX-101 \\ August 1987}
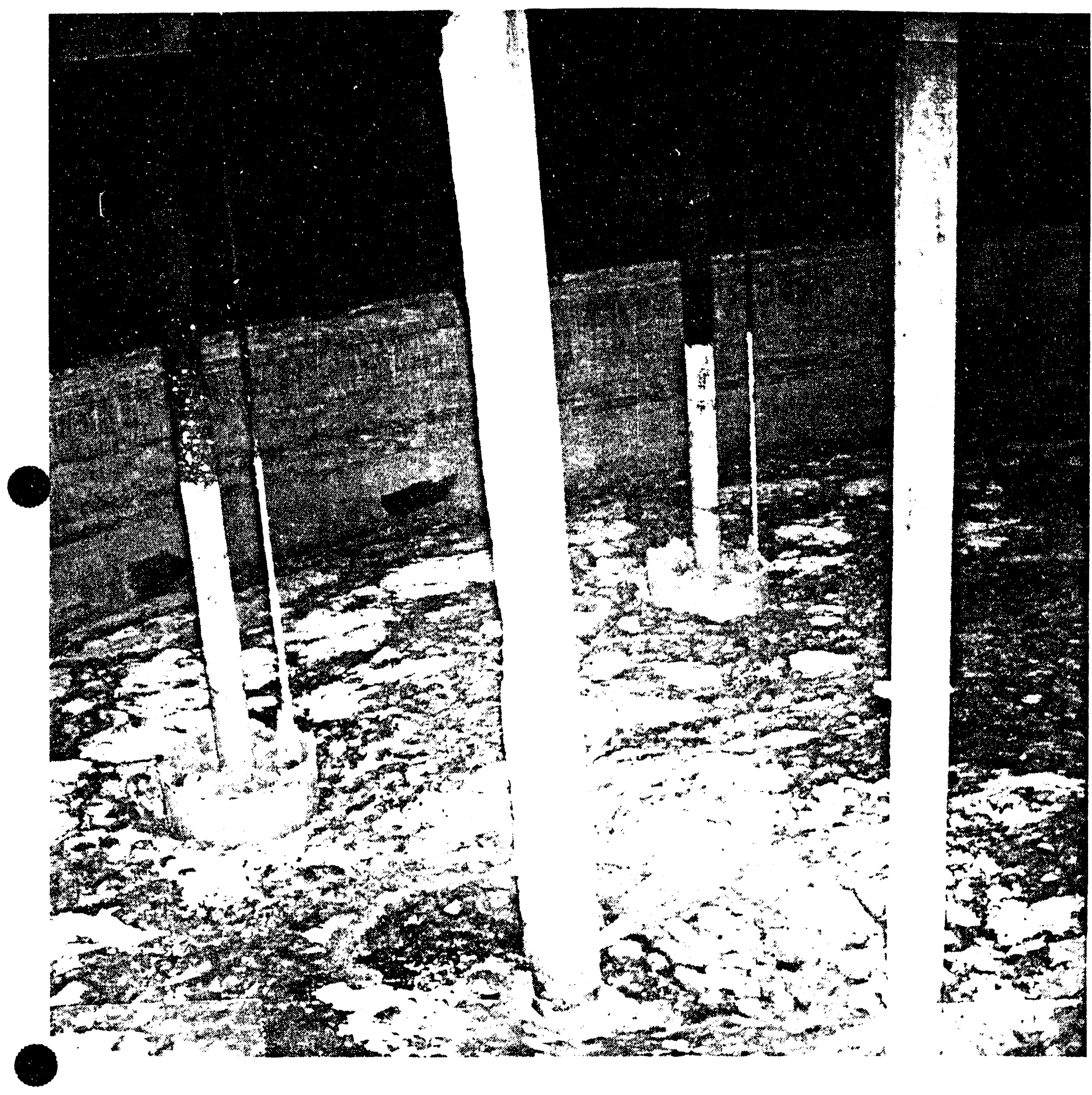
WHC-EP-0625

This page intentionally left blank. 


\section{Tank 241-AX-102}

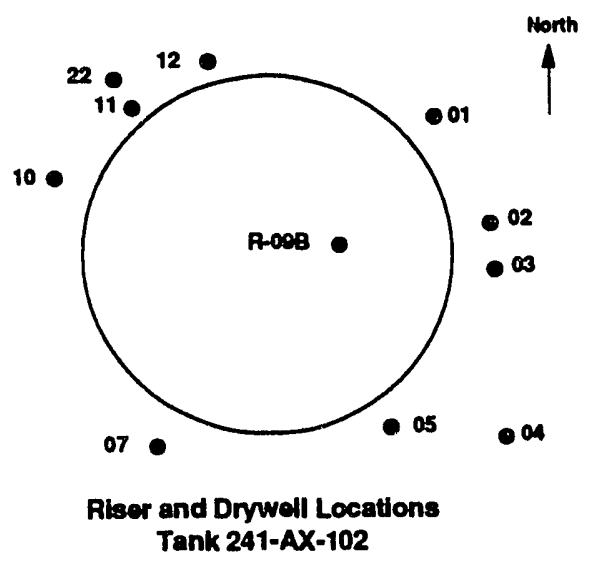

Number of External Drywells: 10

Number of Lateral Wells: None
Tank Description

Type: Single Shell

Constructed: 1964

In-service: 1966

Out of Service: 09/08/80

Diameter: $7^{\circ}$

Usable Depth: 30.3'

Capacity: $1,000 \mathrm{~K}$ gallons

Bottom shape: Flat

Hanford Coordinates:

41.629' North

47.475' West

Ventilation: Passive

\section{Leak Detection System}

Surface Level:

FIC Riser- None

Manual Tape Rtser- R-09B

LOW Riser(s)- None

\section{Tank Status}

Contents

Type: Complexant Concentrate Waste

Total Waste: $39 \mathrm{~K}$ gallons

Supernate Volume: 3K gallons

Drainable Interstitial Liquid: $14 \mathrm{~K}$ gallons

Isolation Status

Date Interim Stabilized: $9 / 88$

Date Interim Isolated: $12 / 15 / 82$

\section{Surface Level/Leak Status}

Integrity Category: Assumed Leaker

Date Declared Confirmed or Assumed Leaker: 1988

Leak Volume Estimate: 3,000 Gallons (1989)

Manual Tape Surface Level: 12.00 Inches (11/10/92)

Last Photographed: 06/05/89

Photo Interpretation Comments:

Photographs reveal a liquid surface and what appears to be a very thin film on the surface.

Temperature Status

Highest recent temperature: $77^{\circ} \mathrm{F}(11 / 20 / 91)$

Comments: Temperatures are stable.

Drywell Status

Comments: Current drywell profiles were stable and consistent with established baseline profiles. 
WHC-EP-0625

This page intentionally left blank. 


\section{Tank 241-AX-103}

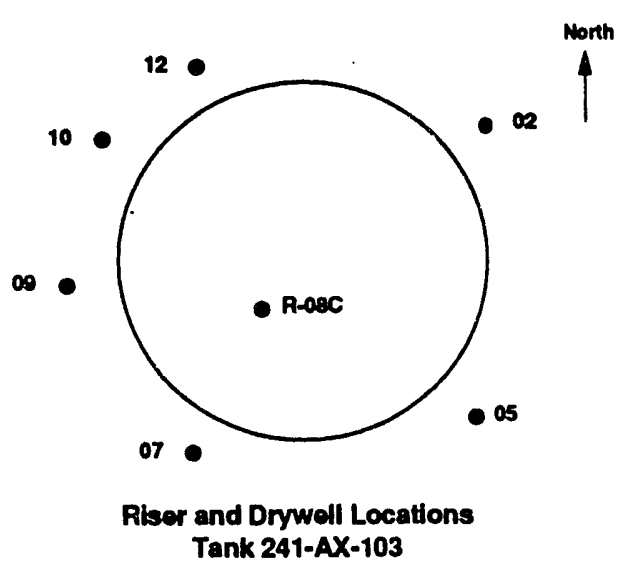

Number of External Drywells: 6

Number of Lateral Wells: None

\section{Tank Status}

Watch List: Hydrogen

Contents

Type: Complexant Concentrate Waste

Total Waste: $112 \mathrm{~K}$ gallons

Supernate Volume: OK gallons

Drainable Interstitial Liquid: $36 \mathrm{~K}$ gallons

\section{Isolation Status}

Date Interim Stabilized: $8 / 87$

Date Partially Interim Isolated: $12 / 15 / 82$

\section{Surface Level/Leak Status}

Integrity Category: Solind

FIC Surface Level: Intrusion level set at 44.50 Inches (12/28/92)

Surface Level Comments: Intrusion Mode

Last Photographed: $08 / 13 / 87$

Photo Interpretation Comments: See attached photo.

Temperature Status

Highest temperature during 1992: $119.7^{\circ} \mathrm{F}(10 / 15 / 92)$

Comments: Temperatures are stable.

\section{Drywell Status}

Comments: Current drywell proflles were stable and consistent with established baseline profiles. 
WHC-EP-0625

This page intentionally left blanik. 
WHC-EP-0625

\section{Tank: 241-AX-103}

\section{August 1987}

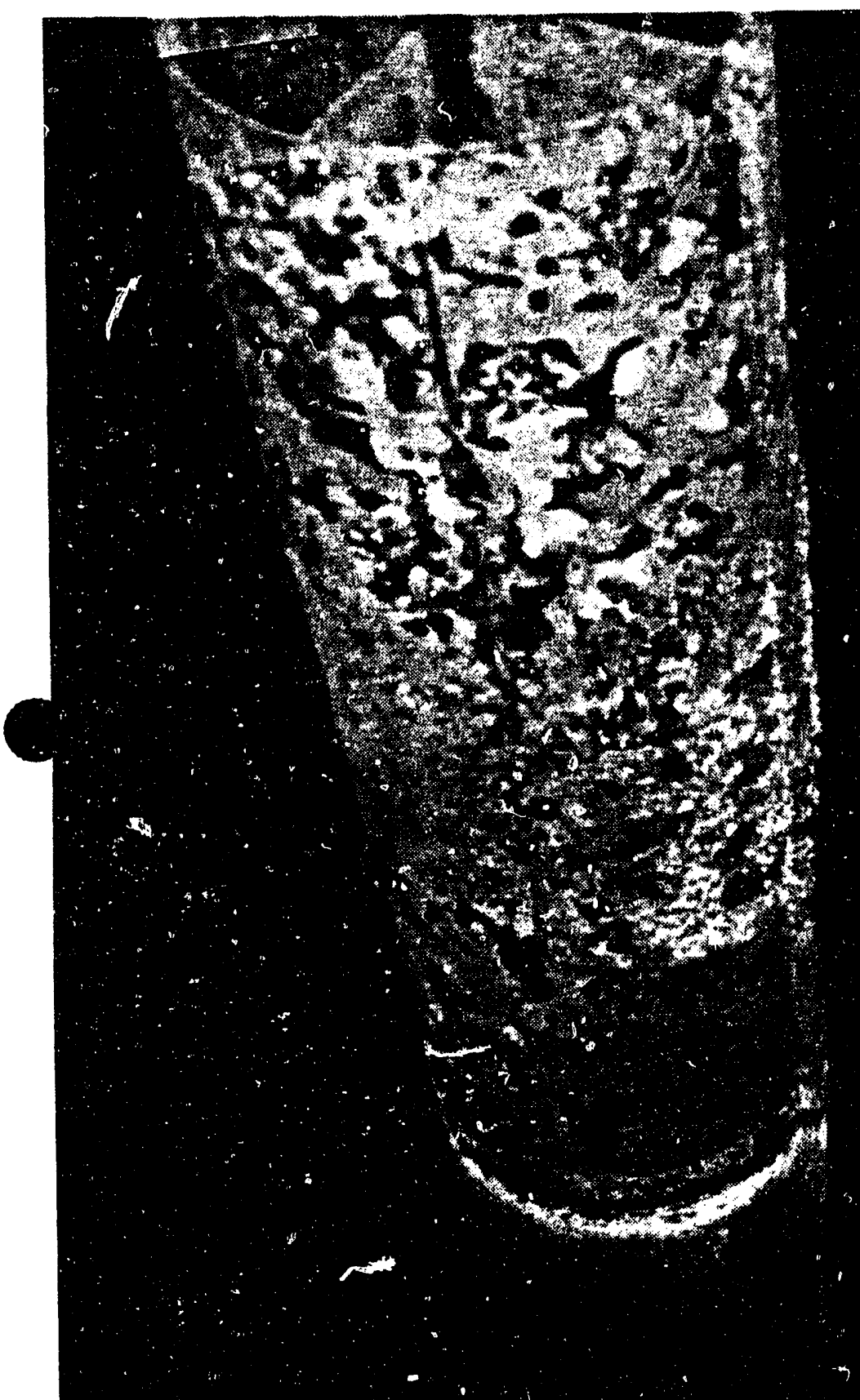

$3 x+4 \times+4$

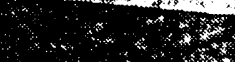

$+\infty$
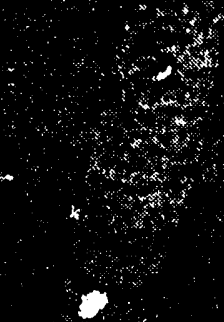

\&

7

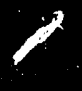


WHC-EP-0625

This page intentionally left blank. 


\section{Tank 241-AX-104}

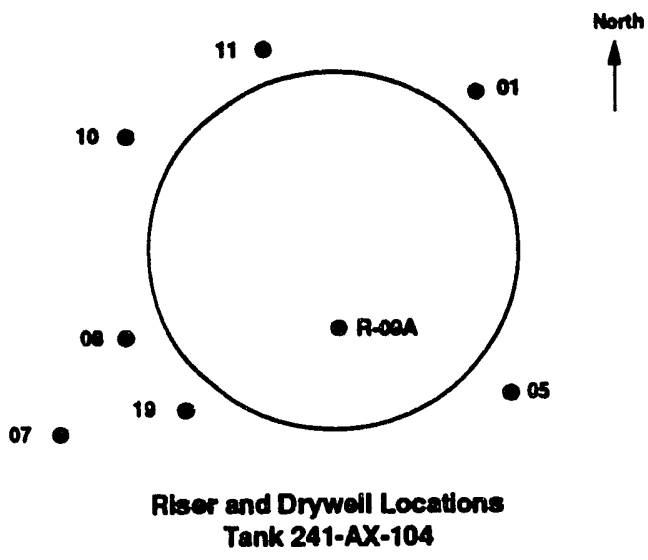

Number of External Drywells: 7 Number of Lateral Wells: None

\section{Tank Status}

Contents

Type: Non-Complexed Waste

Total Waste: $7 \mathrm{~K}$ gallons

Supernate Volume: OK gallons

Drainable Interstitial Liquid: OK gallons

Isolation Status

Date Interim Stabilized: $08 / 10 / 81$

Date Interim Isolated: $12 / 15 / 82$

\section{Surface Level/Leak Status}

Integrity Category: Assumed Leaker

Date Declared Confirmed or Assumed Leaker: 1977

Leak Volume Estimate: 7.8 Gallons $=1 / 19$ th of $150 \mathrm{~K}$ gallons

Manual Tape Surface Level: 2.75 Inches (11/19/92)

Last Photographed: 08/18/87

Photo Interpretation Comments: Photographs reveal a dry, empty tank.

Temperature Status

Highest temperature during $1992: 101.8^{\circ} \mathrm{F}(10 / 15 / 92)$

Comments: Temperatures are stable.

Drywell Status

Comments: Current drywell profiles were stable and consistent with established baseline profiles 
WHC-EP-0625

This page intentionally left blank. 


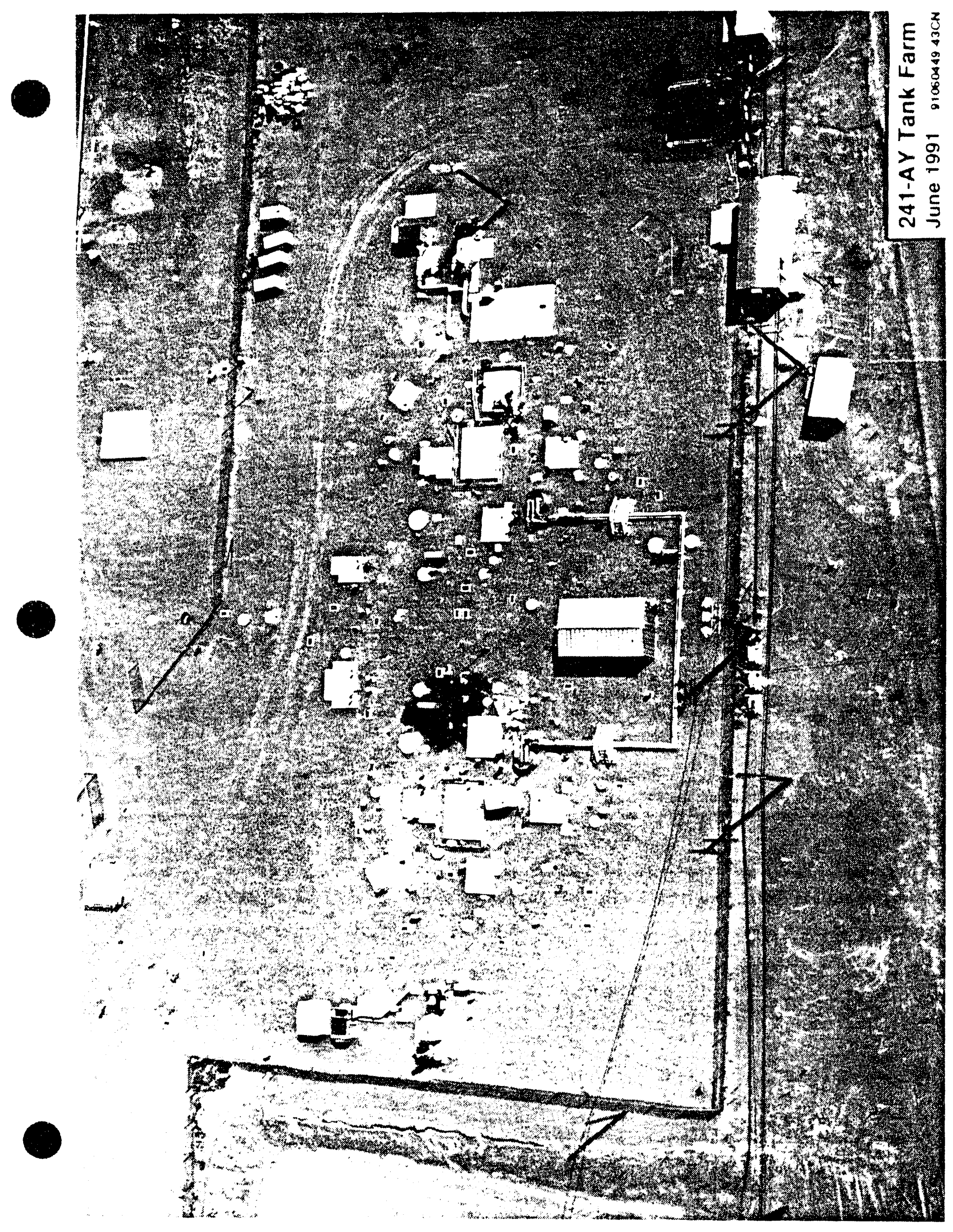


WHC-EP-0625

This page intentionally left blank. 


\section{Tank 241-AY-101}

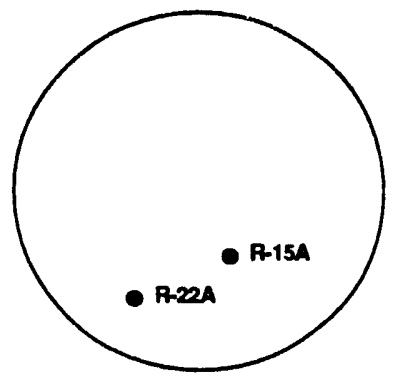

Riser and Drywell Locations Tank 2A1-AY-101

Number of External Drywells: None Number of Lateral Wells: None

\section{Tank Status}

\section{Contents}

Type: Dilute Complexed Waste

Total Waste: $928 \mathrm{~K}$ gallons

Supernate Volume: $845 \mathrm{~K}$ gallons

Drainable Interstitial Liquid: $2 \mathrm{~K}$ gallons

Surface Level/Leak Status

Integrity Category: Sound

Manual Tape Surface Level: 334.50 Inches (12/29/92)

Last Photographed: 12/28/82

Photo Interpretation Comments: No current photographs.

Temperature Status

Highest temperature during 1992: 
WHC-EP-0625

This page intentionally left blank. 


\section{Tank 241-AY-102}

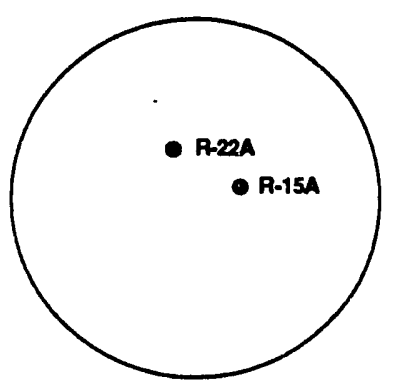

Riser and Drywell Locations Tank 241-AY-102

Number of External Drywells: None Number of Lateral Wells: None

\section{Tank Status}

Contents

Type: Dllute Non-Complexed Waste

Total Waste: $574 \mathrm{~K}$ gallons

Supernate Volume: $542 \mathrm{~K}$ gallons

Drainable Interstitial Liquid: OK gallons

\section{Surface Level/Leak Status}

Integrity Category: Sound

Manual Tape Surface Level: 237.50 Inches (12/29/92)

FIC Surface Level: 234.40 Inches (12/29/92)

Last Photographed: 04/28/81

Photo Interpretation Comments: No current photographs.

\section{Temperature Status}

Highest temperature during 1992:
Tank Description

Type: Double Shell

Constructed: 1970

In-service: 1976

Diameter: $7^{\circ}$

Usable Depth: 30.4'

Capacity: 1,000K gallons

Bottom shape: Flat

Hanford Coordinates:

41,626.06' North

47.836.54' West

Air Lift Circulators: Yes (22)

Ventilation: Operating Exhauster

\section{Leak Detection System}

Surface Level:

FIC Riser- R-22A

Manual Tape Riser- R-15A

LOW Riser(s)- None 
WHC-EP-0625

This page intentionally left blank. 


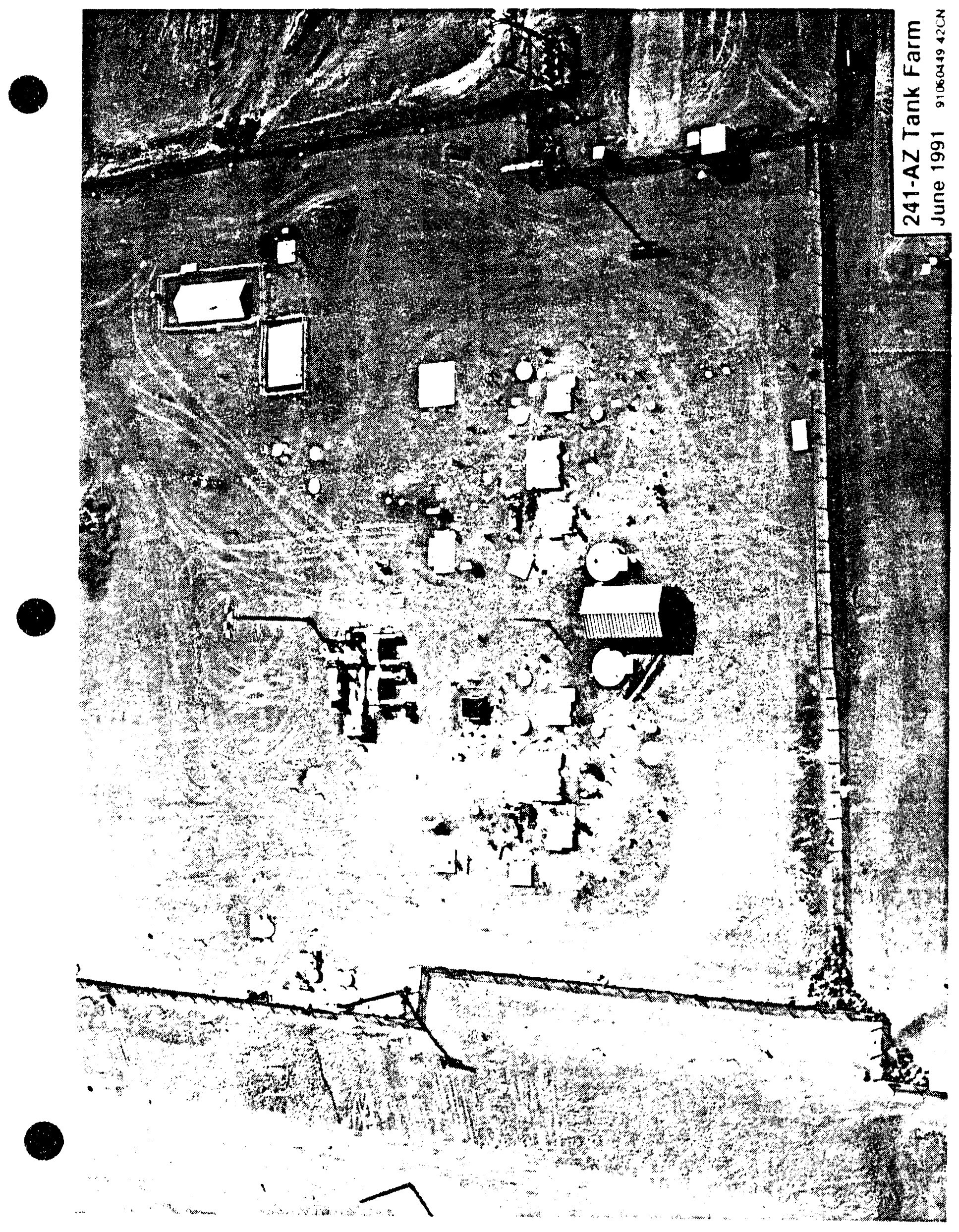




\section{WHC-EP-0625}

This page intentionally left blank. 


\section{Tank 241-AZ-101}

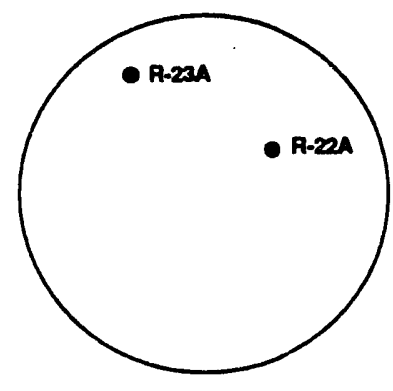

Risor and Drywell Locations Tank 241-AZ-101

Number of External Drywells: None Number of Lateral Wells: None

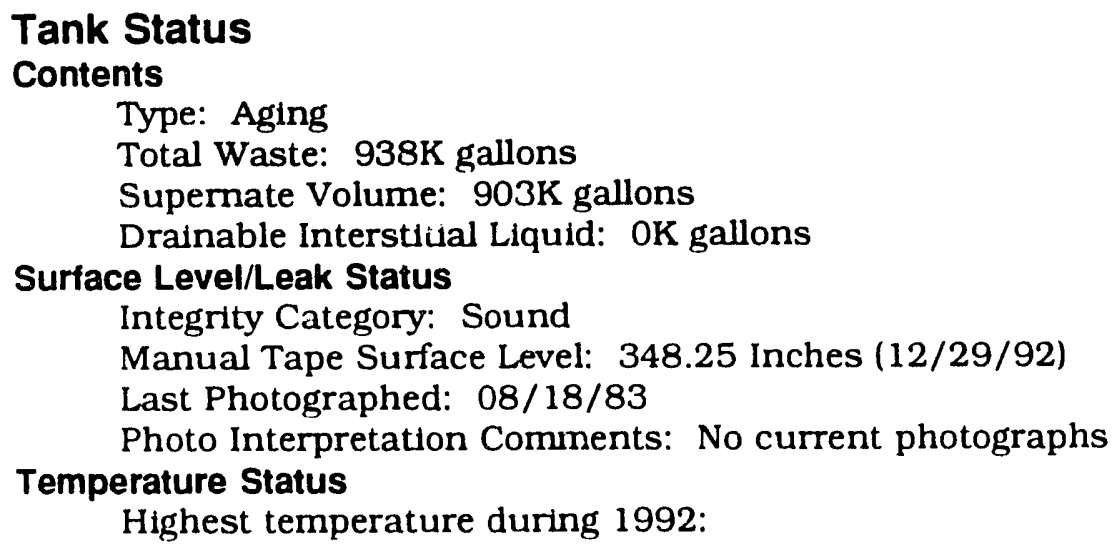


WHC-EP-0625

This page intentionally left blank. 


\section{Tank 241-AZ-102}

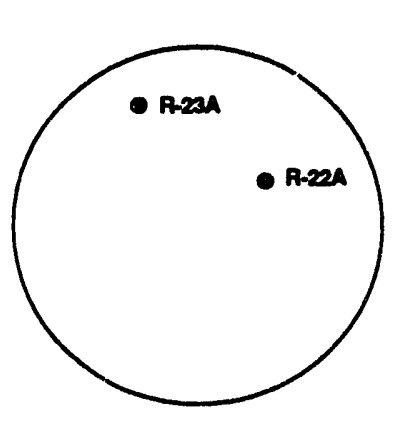

Riser and Drywell Locations Tank 241-AZ-102

Number of External Drywells: Non:

Tank Description Type: Double Shell Constructed: 1977

In-service: 1975

Diameter: $75^{\circ}$

Usable Depth: 30.4'

Capactty: 1,000K gallons

Bottom shape: Flat

kinnford Coordinates:

42,080' North

47,613' West

Air Lift Circulators: Yes (22)

Ventilation: Operating Exhauster

\section{Leak Detection System}

Surface Level:

FIC Riser- R-22A

Manual Tape Riser- R-23A

LOW Riser(s)- None

Number of Lateral Wells: None

\section{Tank Status \\ Contents}

Type: Aging

Total Waste: $945 \mathrm{~K}$ gallons

Supernate Volume: $850 \mathrm{~K}$ gallons

Drainable Interstitial Liquid: $4 \mathrm{~K}$ gallons

Surface Level/Leak Status

Integrity Category: Sound

Manual Tape Surface Level: 337.25 Inches (12/29/92)

Last Photographed: 12/24/84

Photo Interpretation Comments: Photographs are not current because of transfers.

Temperature Status

Highest temperature during 1992: 
WHC-EP-0625

This page intentionally left blank. 
WHC-EP-0625

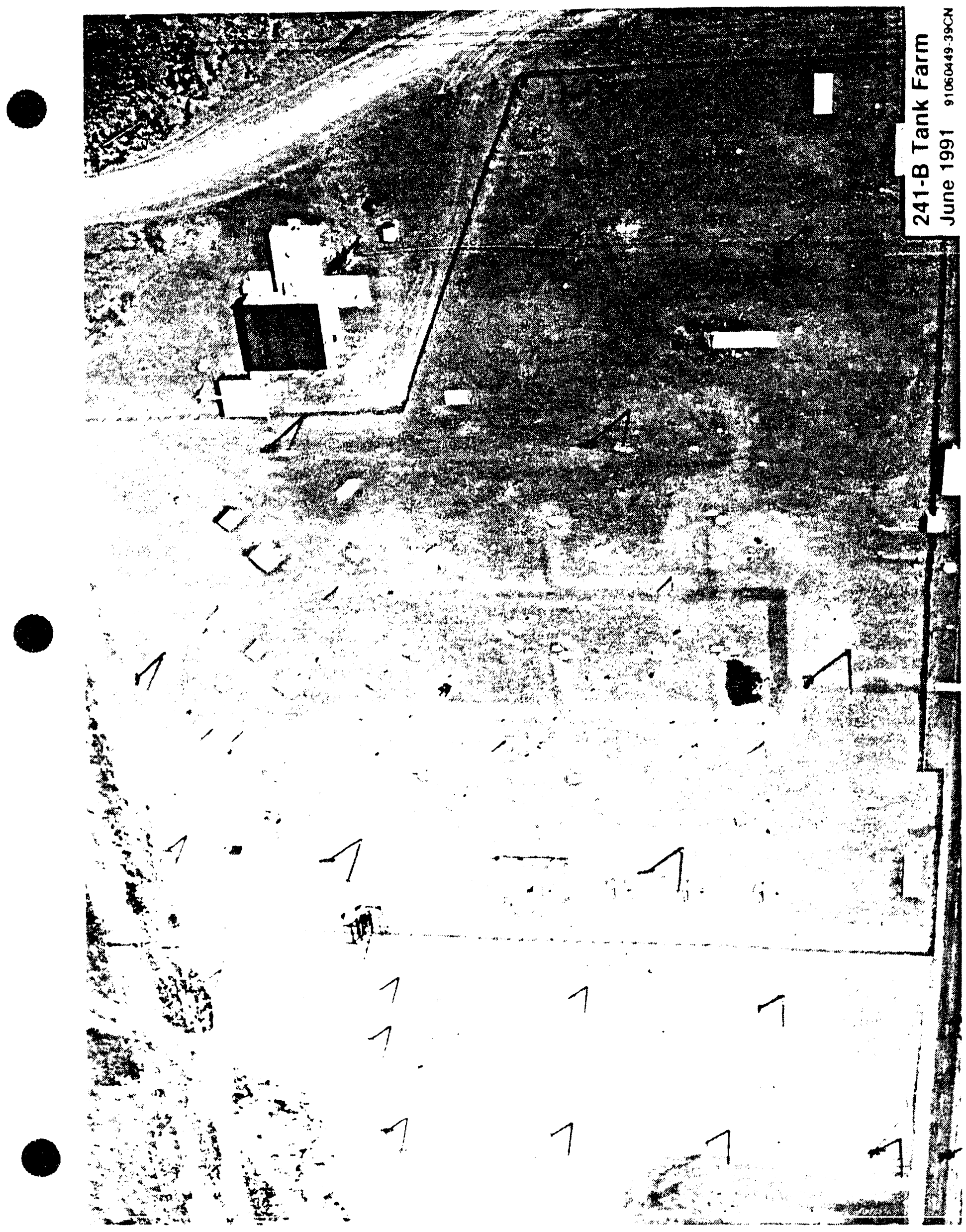


WHC-EP-0625

This page intentionally left blank. 


\section{Tank 241-B-101}

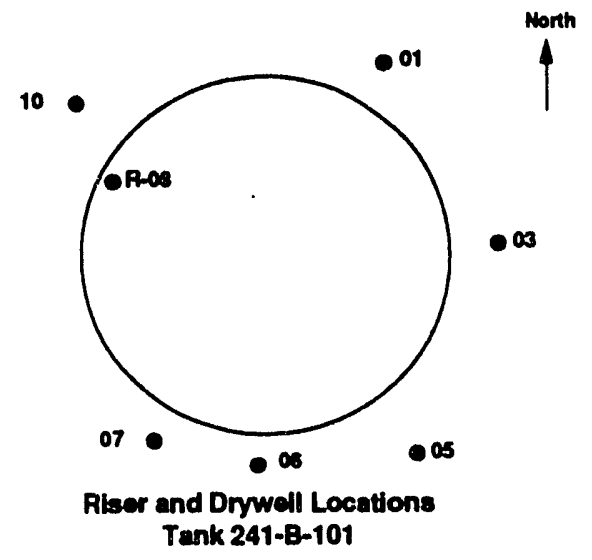

Number of External Drywells: 6

Number of Lateral Wells: None

\section{Tank Status}

\section{Contents}

Type: Non-Complexed Waste

Total Waste: 113K gallons

Supernate Volume: OK gallons

Drainable Interstitial Liquid: $6 \mathrm{~K}$ gallons

Isolation Status

Date Interim Stabilized: $03 / 81$

Date Interim Isolated: 05/81

\section{Surface Level/Leak Status}

Integrity Category: Assumed Leaker

Date Declared Confirmed or Assumed Leaker: 1974

Leak Volume Estimate: $7.8 \mathrm{~K}$ gallons $=1 / 19$ th of $150 \mathrm{~K}$ gallons

FIC Surface Level: Intrusion level set at 36.70 Inches (12/21/92)

Surface Level Comments: Intrusion Mode

Last Photographed: 05/19/83

Photo Interpretation Comments:

Although some of the $05 / 19 / 83$ photographs are hazy, the visible surface is sludge with no surface liquid. The FIC plummet is not in view and there appears to be little change from photographs taken $9 / 22 / 77$.

\section{Temperature Status}

Highest temperature during 1992: $111.3^{\circ} \mathrm{F}(01 / 04 / 92)$

Comments: Temperatures are stable.

\section{Drywell Status}

Comments: Current drywell profiles were stable and consistent with established baseline profiles. 
WHC-EP-0625

This page intentionally left blank. 


\section{Tank 241-B-102}

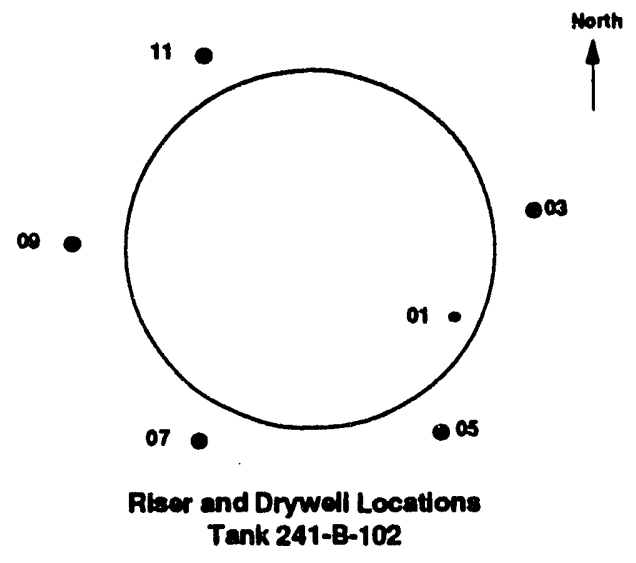

Number of External Drywells: 5

Number of Lateral Wells: None

\section{Tank Status}

\section{Contents}

Type: Non-Complexed Waste

Total Waste: $32 \mathrm{~K}$ gallons

Supernate Volume: 4K gallons

Drainable Interstitial Liquid: OK gallons

Isolation Status

Date Interim Stabllized: 08/23/85

Date Interim Isolated: 09/25/85

Surface Level/Leak Status

Integrity Category: Sound

FIC Surface Level: 7.10 Inches (12/21/92)

Last Photographed: 08/22/85

Photo Interpretation Comments:

Photographs from 05/20/83 show that the FIC plummet is contacting liquid.

There appears to be more liquid than in prevlous photographs. Photographs from $8 / 22 / 85$ taken aftei pumping show a very shallow liquid surface and exposed

Temperature Status solids around the perimeter of the tank. The FIC plummet is contacting solids.

Highest temperature during 1992: $75.6^{\circ} \mathrm{F}(01 / 04 / 92)$

Comments: Temperatures are stable.

Drywell Status

Comments: Current drywell proflles were stable and consistent with established baseline profiles. 
WHC-EP-0625

This page intentionally left blank. 


\section{Tank 241-B-103}

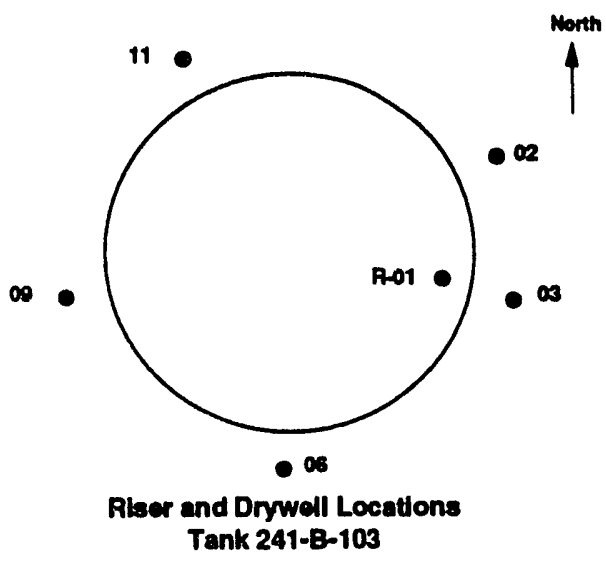

Number of External Drywells: 5

Number of Lateral Wells: None

Tank Status

Watch LIst: Organic Salts

Contents

Type: Non-Complexed Waste

Total Waste: $59 \mathrm{~K}$ gallons

Supernate Volume: OK gallons

Drainable Interstitial Liquid: OK gallons

Isolation Status

Date Interm Stabilized: 02/26/85

Date Interim Isolated: 09/25/85

\section{Surface Level/Leak Status}

Integrity Category: Assumed Leaker

Date Declared Confirmed or Assumed Leaker: 1978

Leak Volume Estimate: $7.8 \mathrm{~K}$ gallons $=1 / 19$ th of $150 \mathrm{~K}$ gallons

FIC Surface Level: Intrusion level set at 17.40 Inches (12/21/92)

Surface Level Comments: Intrusion Mode

Last Photographed: 02/05/85

Photo Interpretation Comments: See attached photo.

Temperature Status

Highest temperature during 1992: $198.6^{\circ} \mathrm{F}(03 / 06 / 92)$

Comments: Temperatures are stable.

Drywell Status

Comments: Current drywell profiles were stable and consistent with established baseline profiles.
Tank Description

Type: Single Shell

Constructed: 1944

In-service: $12 / 45$

Depth: 16

Bottom shape: Dish

Vent

\section{Leak Detection System}

Surface Level:

FIC Riser- R-01

Manual Tape Riser- None

LOW Riser(s)- None 
WHC-EP-0625

This page intentionally left blank. 


\section{Tank: 241-B-103 \\ October 1988}

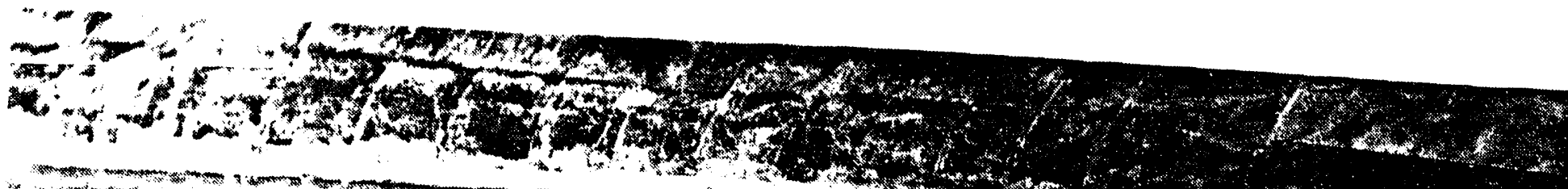

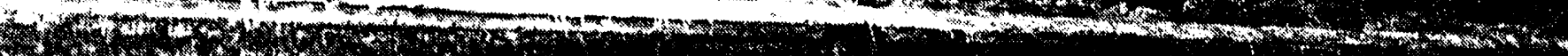

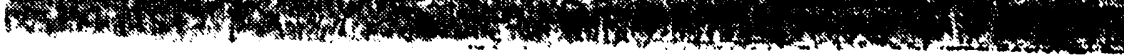

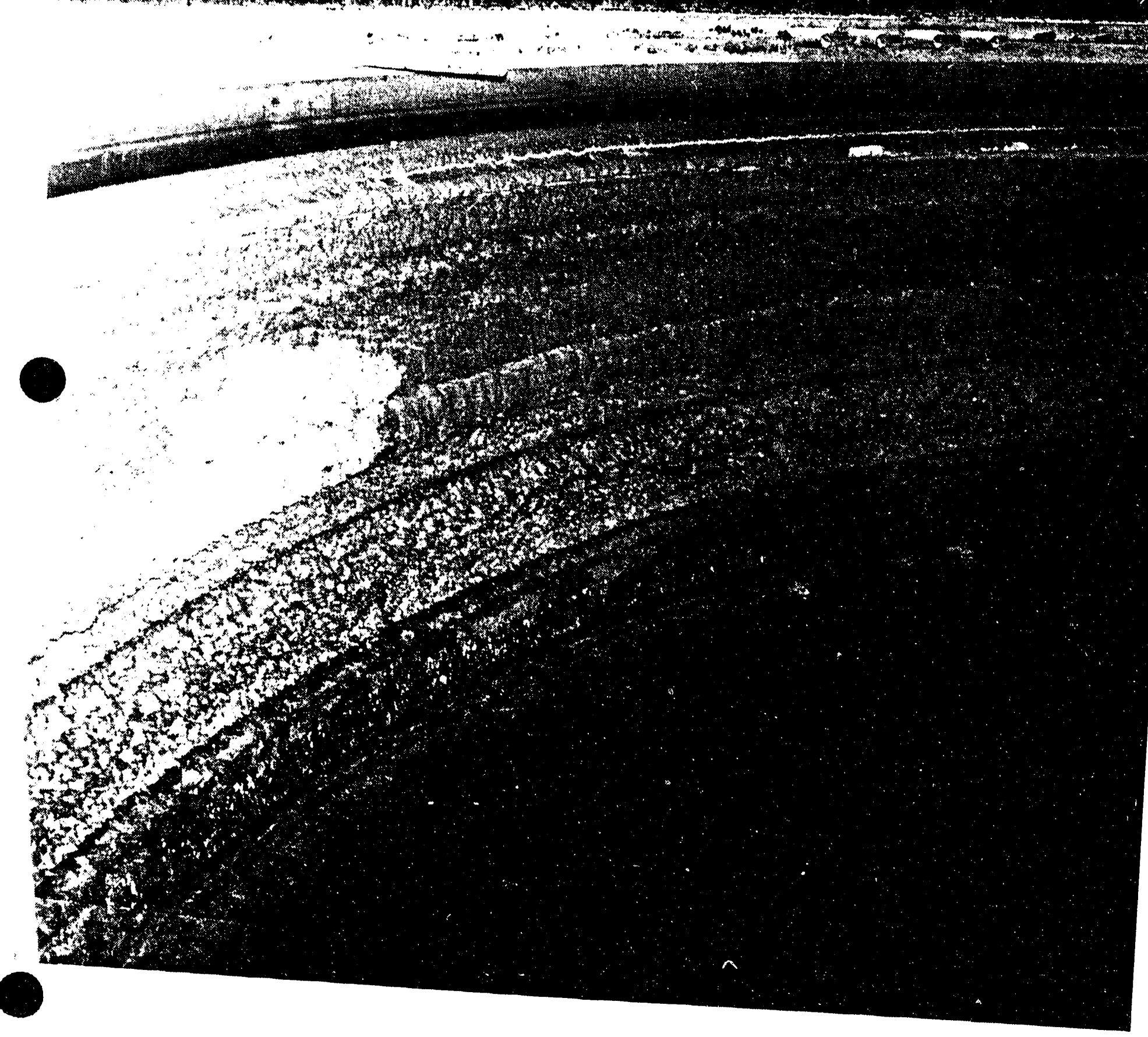




\section{WHC-EP-0625}

This page intentionally left blank. 


\section{Tank 241-B-104}

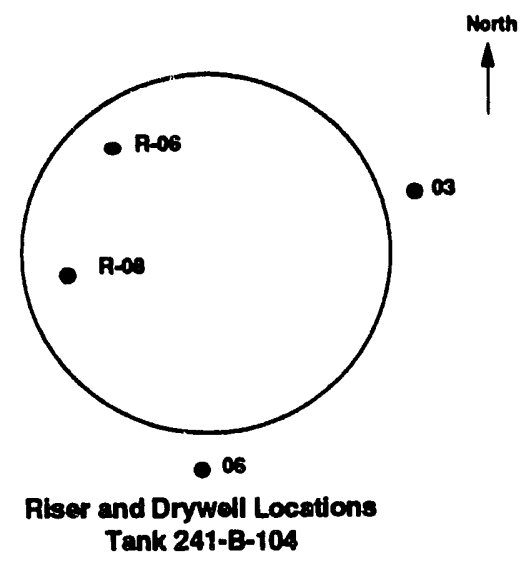

Tank 241-B-104
Tank Description

Type: Single Shell

Constructed: 1944

In-service: $08 / 46$

Diameter: $75^{\prime}$

Usable Depth: $16^{\circ}$

Capactty: $530 \mathrm{~K}$ gallons

Bottom shape: Dish

Hanford Coordinates:

$45,237^{\circ}$ North

52,562' West

Ventllation: Passive

\section{Leak Detection System}

Surface Level:

FIC Riser- None

Manual Tape Riser- R-08

LOW Riser(s)- R-06

Number of External Drywells: 2

Number of Lateral Wells: None

\section{Tank Status}

Contents

Type: Non-Complexed Waste

Total Waste: $371 \mathrm{~K}$ gallons

Supernate Volume: $1 \mathrm{~K}$ gallons

Drainable Interstitial Liquid: $46 \mathrm{~K}$ gallons

\section{Isolation Status}

Date Interim Stabilized: $06 / 28 / 85$

Date Interim Isolated: 09/25/85

\section{Surface Level/Leak Status}

Integrity Category: Sound

Manual Tape Surface Level: 132.00 Inches (11/23/92)

Last Photographed: $10 / 13 / 88$

Photo Interpretation Comments:

Photographs taken after pumping to a minimum heel show a shallow liquid surface with exposed solids, many of which are islands. Crystal growth is evident on most of the solids. Measurement anomalies should be expected from discarded tapes and liquid and crystal growth in the immediate area of the manual tape

Temperature Status doughnut plummet.

Highest temperature during 1992: $65.7^{\circ} \mathrm{F}(01 / 04 / 92)$

Comments: Temperatures are stable.

\section{Drywell Status}

Comments: Current drywell profiles were stable and consistent with established baseline profiles. 
WHC-EP-0625

This page intentionally left blank. 


\section{Tank 241-B-105}

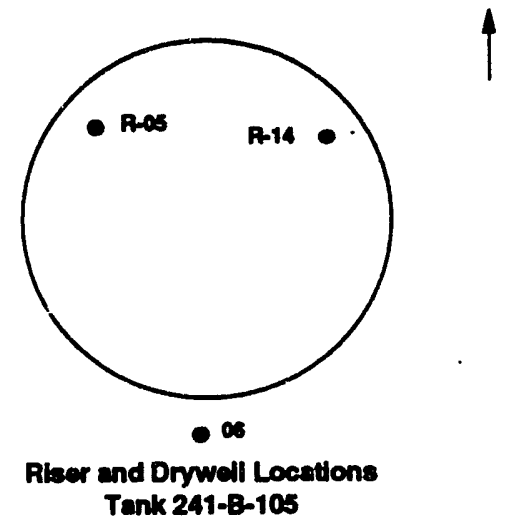

Number of External Drywells: 1

Number of Lateral Wells: None
Tank Description

Type: Single Shell Constructed: 1944

In-service: $01 / 47$

Diameter: $7^{\circ}$

Usable Depth: 16'

Capacity: $530 \mathrm{~K}$ gallons

Bottom shape: Dish

Hanford Coordinates: 45.337' North 52.562' West

Ventilation: Passive

\section{Leak Detection System}

Surface Level:

FIC Riser- None

Manual Tape Riser- R-14

LOW Riser(s)- R-05

\section{Tank Status \\ Contents}

Type: Non-Complexed Waste

Total Waste: $306 \mathrm{~K}$ gallons

Supernate Volume: OK gallons

Isolation Status

Drainable Interstitial Liquid: $23 \mathrm{~K}$ gallons

Date Interim Stabilized: $12 / 27 / 84$

Date Interim Isolated: 10/30/85

\section{Surface Level/Leak Status}

Integrity Category: Assumed Leaker

Date Declared Confirmed or Assumed Leaker: 1978

Leak Volume Estimate: $7.8 \mathrm{~K}$ gallons $=1 / 19$ th of $150 \mathrm{~K}$ gallons

Manual Tape Surface Level: 41.50 Inches (12/18/92)

Last Photographed: 05/19/88

Photo Interpretation Comments:

Photographs from $02 / 12 / 82$ show heavy rust patterns on the side wall. The salt cake is collapsed and heavy salt formations and crystal growth are visible. A hole around the salt well contains liquid. Photographs from 12/18/84 show no change from previous photos.

\section{Temperature Status}

Highest temperature during 1992: $70.40^{\circ} \mathrm{F}(11 / 06 / 92)$

Comments: Temperatures are stable.

Drywell Status

Comments: Current drywell profiles were stable and consistent with established baseline profiles. 
WHC-EP-0625

This page intentionally left blank. 


\section{Tank 241-B-106}

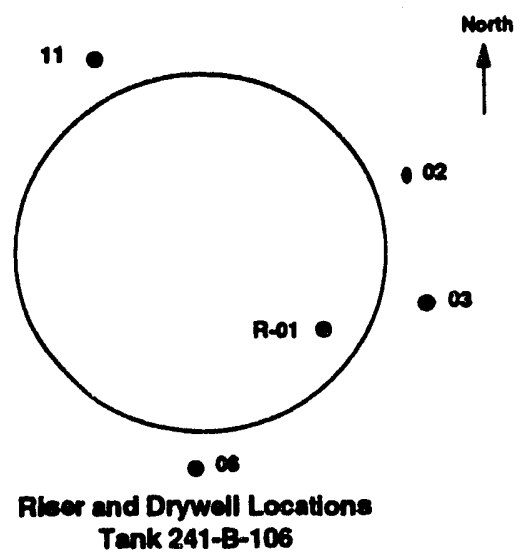

Number of External Drywells: 4

Number of Lateral Wells: None

\section{Tank Status}

Contents

Type: Non-Complexed Waste

Total Waste: $117 \mathrm{~K}$ gallons

Supernate Volume: $1 \mathrm{~K}$ gallons

Drainable Interstitial Liquid: $6 \mathrm{~K}$ gallons

Isolation Status

Date Interim Stabilized: $03 / 20 / 85$

Date Interim Isolated: $10 / 30 / 85$

Surface Level/Leak Status

Integrity Category: Sound

FIC Surface Level: 39.10 Inches (12/21/92)

Last Photographed: 02/28/85

Photo Interpretation Cormments:

Photographs show a smooth surface of solids with some shallow liquid visible. The FIC plummet is contacting liquid near the edge of a very shallow pool. Some measurement anomalies may be experienced.

Temperature Status

Highest temperature during 1992: $64.3^{\circ} \mathrm{F}(01 / 01 / 92)$

Comments: Temperatures are stable.

Drywell Status

Comments: Current drywell profiles were stable and consistent with established baseline profules.
Tank Description

Type: Single Shell

Constructed: 1944

In-service: $09 / 47$

Diameter: $75^{\prime}$

Usable Depth: $16^{\prime}$

Capacity: $530 \mathrm{~K}$ gallons

Bottom shape: Dish

Hanford Coordinates:

45,357' North

52,562' West

Ventilation: Passive

\section{Leak Detection System \\ Surface Level: \\ FIC Riser- R-01 \\ Manual Tape Riser- None \\ LOW Riser(s)- None}




\section{WHC-EP-0625}

This page intentionally left blank. 


\section{Tank 241-B-107}

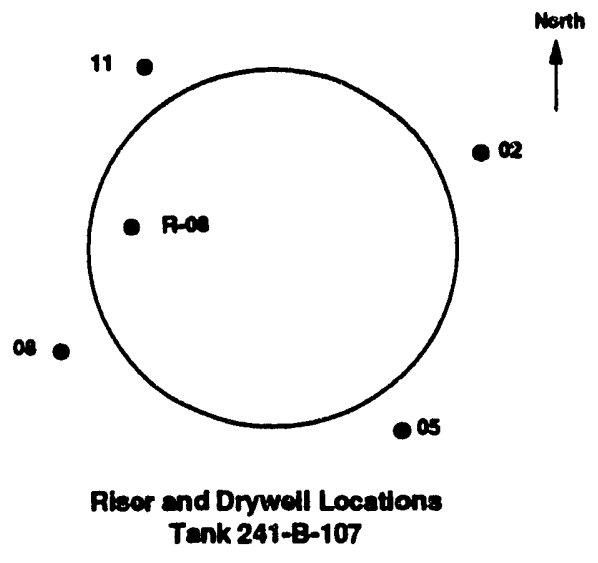

Number of External Drywells: 4

Number of Lateral Wells: None

\section{Tank Status}

Contents

Type: Non-Complexed Waste

Total Waste: $165 \mathrm{~K}$ gallons

Supernate Volume: $1 \mathrm{~K}$ gallons

Drainable Interstitial Liquid: $12 \mathrm{~K}$ gallons

Isolation Status

Date Partially Interim Isolated: $12 / 15 / 82$

Date Interim Stabilized: 03/20/85

Date Interim Isolated: $10 / 30 / 85$

Surface Level/Leak Status

Integrity Category: Assumed Leaker

Date Declared Confirmed or Assumed Leaker: 1980

Leak Volume Estimate: 8,000 Gallons (1986)

Manual Tape Surface Level: 54.50 Inches (11/23/92)

Last Photographed: $02 / 28 / 85$

Photo Interpretation Comments:

Photographs show a solid surface and several areas with crystal growth. There are some small pockets of shallow liquid visible. The manual tape pencil plummet is contacting sludge and a discarded tape is in the immediate area.

\section{Temperature Status}

Highest temperature during 1992: $68.70^{\circ} \mathrm{F}(11 / 06 / 92)$

Comments: Temperatures are stable.

Drywell Status

Comments: Current drywell proflles were stable and consistent with established baseline proflles. 
WHC-EP-0625

This page intentionally left blank. 


\section{Tank 241-B-108}

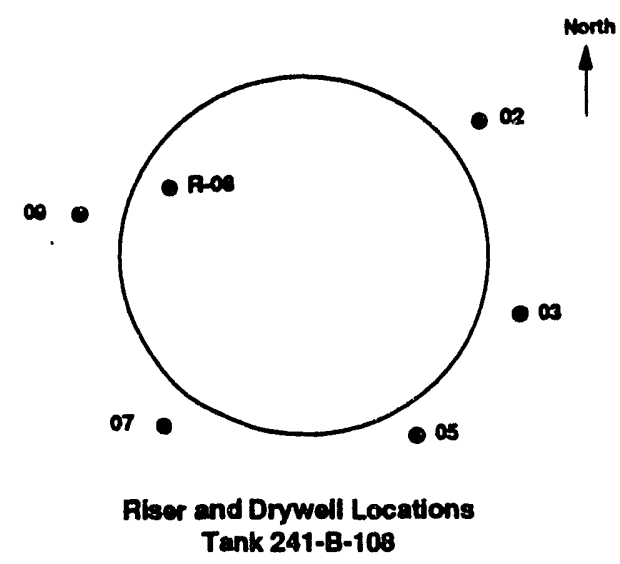

Number of External Drywells: 5

Number of Lateral Wells: None

\section{Tank Status}

Contents

Type: Non-Complexed Waste

Total Waste: $94 \mathrm{~K}$ gallons

Supernate Volume: OK gallons

Drainable Interstitial Liquid: $4 \mathrm{~K}$ gallons

Isolation Status

Date Interim Stabilized: 05/22/85

Date Interim Isolated: $10 / 28 / 85$

\section{Surface Level/Leak Status}

Integrity Category: Sound

FIC Surface Level: Intrusion level set at 30.30 Inches (12/21/92)

Surface Level Comments: Intrusion Mode

Last Photographed: 05/10/85

Photo Interpretation Comments:

Photographs show a surface of sludge. The FIC plummet is contacting solids and a discarded tape is in the immediate area of the plummet.

\section{Temperature Status}

Highest temperature during 1992: $65.4^{\circ} \mathrm{F}(01 / 01 / 92)$

Comments: Temperatures are stable.

\section{Drywell Status}

Comments: Current drywell profiles were stable and consistent with established baseline profiles. 
WHC-EP-0625

This page intentionally left blank. 


\section{Tank 241-B-109}

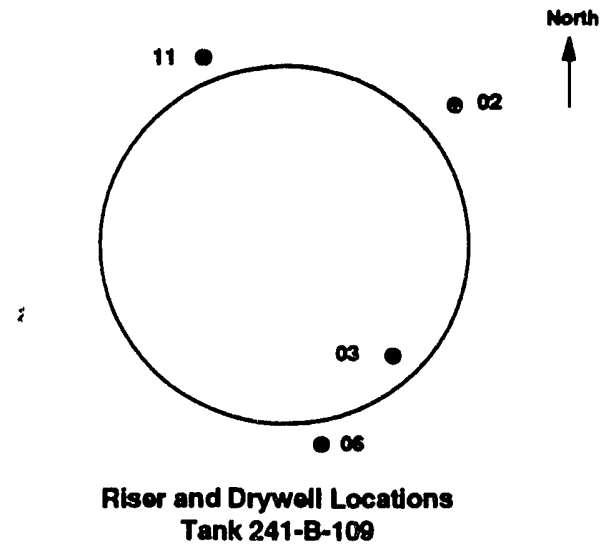

Tank 241-B-100
Tank Description

Type: Single Shell

Constructed: 1944

In-service: $01 / 46$

Diameter: $7^{\circ}$

Usable Depth: 16'

Capacity: $530 \mathrm{~K}$ gallons

Bottom shape: Dish

Hanford Coordinates:

45.437' North

52,752 ' West

Ventilation: Passive

\section{Leak Detection System}

Surface Level:

FIC Riser- None

Manual Tape Riser- R-03

LOW Riser(s)- None

Number of External Drywells: 3

Number of Lateral Wells: None

\section{ank Status}

ntents

Type: Non-Complexed Waste

Total Waste: $127 \mathrm{~K}$ gallons

Supernate Volume: OK gallons

Drainable Interstitial Liquid: $8 \mathrm{~K}$ gallons

Isolation Status

Date Interim Stabilized: 04/09/85

Date Interim Isolated: 10/30/85

\section{Surface Level/Leak Status}

Integrity Category: Sound

Manual Tape Surface Level: 41.00 Inches (11/23/92)

Last Photographed: 04/02/85

Photo Interpretation Comments:

Photographs reveal a surface of damp sludge and dry exposed solids along the outer edge. The manual tape doughnut plummet is contacting dry solids in a hole several feet deep.

Temperature Status

Highest temperature during 1992: $65^{\circ} \mathrm{F}(01 / 01 / 92)$

Comments: Temperatures are stable.

\section{Drywell Status}

Comments: Current drywell profiles were stable and consistent with established baseline profiles. 


\section{WHC-EP-0625}

This page intentionally left blank. 


\section{Tank 241-B-110}

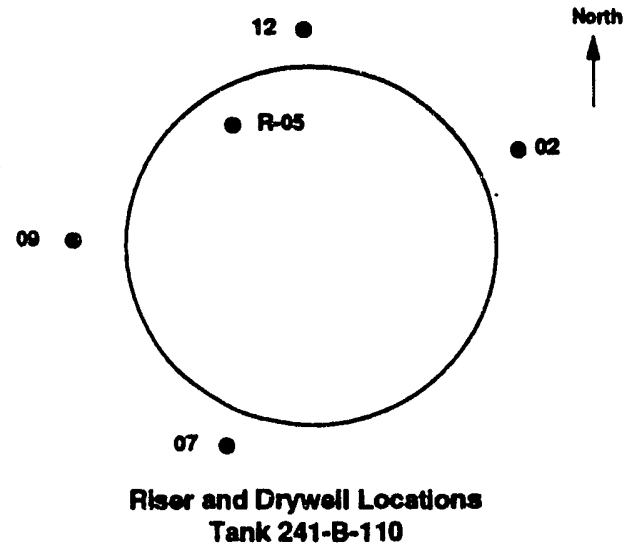

Number of External Drywells: 4

Number of Lateral Wells: None

\section{Tank Status}

Contents

Type: Non-Complexed Waste

Total Waste: $246 \mathrm{~K}$ gallons

Supernate Volume: 1K gallons

Drainable Interstitial Liquid: $22 \mathrm{~K}$ gallons

Isolation Status

Date Interim Stabilized: $12 / 20 / 84$

Date Interim Isolated: 10/30/85

\section{Surface Level/Leak Status}

Integrity Category: Assumed Leaker

Date Declared Confirmed or Assumed Leaker: 1981

Leak Volume Estimate: 10,000 Gallons (1986)

Manual Tape Surface Level: 86.00 Inches (11/23/92)

Last Photographed: $03 / 17 / 88$

Photo Interpretation Comments:

Photographs from 01/09/81 and 12/13/84 show a caked, cracked, level surface of solids with a large pool of liquid in the center of the tank. The manual tape

Temperature Status plummet is contacting solids.

Highest temperature during 1992: $70.5^{\circ} \mathrm{F}(02 / 07 / 92)$

Comments: Temperatures are stable.

\section{Drywell Status}

Comments: Current drywell profiles were stable and consistent with established baseline proflles.
Tank Description

Type: Single Shell

Constructed: 1944

In-service: $05 / 45$

Out of Service: 1970

Diameter: $7^{\circ}$

Usable Depth: $16^{\prime}$

Capacity: $530 \mathrm{~K}$ gallons

Bottom shape: Dish

Hanford Coordinates:

$45,327^{\prime}$ North

52,852' West

Ventllation: Passive

\section{Leak Detection System}

Surface Level:

FIC Riser- None

Manual Tape Riser- R-05

LOW Riser(s)- None 
WHC-EP-0625

This page intentionally left blank. 


\section{Tank 241-B-111}

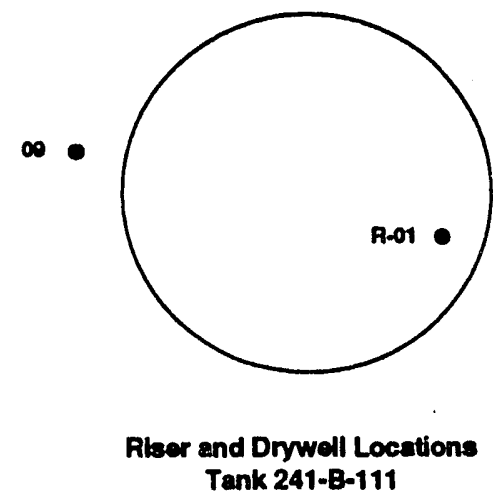

Number of External Drywells: 1

Number of Lateral Wells: None
Tank Description

Type: Single Shell

Constructed: 1944

In-service: $11 / 45$

Diameter. $7^{\circ}$

Usable Depth: 16'

Capacity: $530 \mathrm{~K}$ gallons

Bottom shape: Dish

Hanford Coordinates:

$45,337^{\prime}$ North

52,852 ' West

Ventilation: Passive

\section{Leak Detection System}

Surface Level:

FIC Riser- R-01

Manual Tape Rtser- None

LOW Riser(s)- None

\section{Tank Status}

\section{Contents}

Type: Non-Complexed Waste

Total Waste: $237 \mathrm{~K}$ gallons

Supernate Volume: $1 \mathrm{~K}$ gallons

Drainable Interstitial Liquid: $21 \mathrm{~K}$ gallons

Isolation Status

Date Interim Stabilized: 06/28/85

Date Interim Isolated: 10/30/85

\section{Surface Level/Leak Status}

Integrity Category: Assumed Leaker

Date Declared Confirmed or Assumed Leaker: 1978

Leak Volume Estimate: $7.8 \mathrm{~K}$ gallons $=1 / 19$ th of $150 \mathrm{~K}$ gallons

FIC Surface Level: Intrusion level set at 84.40 Inches (12/29/92)

Surface Level Comments: Intrusion Mode

Last Photographed: 06/26/85

Photo Interpretation Comments:

Photographs show a very shallow liquid surface toward one side of tank and exposed sludge on the other side where the FIC is located. The FIC plummet is

Temperature Status contacting sludge.

Highest temperature during 1992: $86^{\circ} \mathrm{F}(07 / 02 / 92)$

Comments: Temperatures are stable.

\section{Drywell Status}

Comments: Current drywell profiles were stable and consistent with established baseline profiles. 
WHC-EP-0625

This page intentionally left blank. 


\section{Tank 241-B-112}

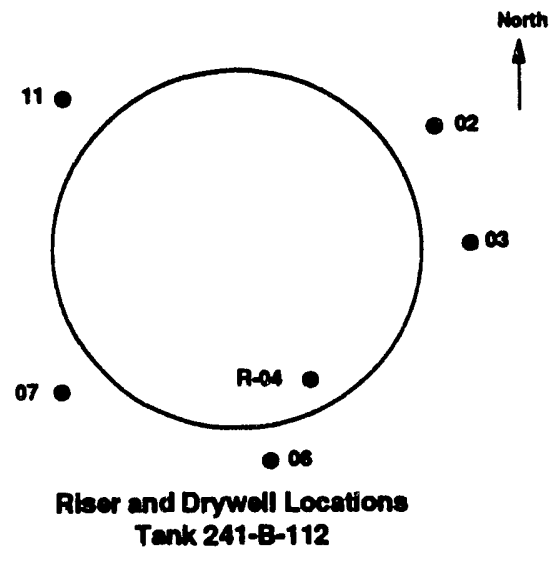

Number of External Drywells: 5

Number of Lateral Wells: None

\section{Tank Status}

Contents

Type: Non-Complexed Waste

Total Waste: $33 \mathrm{~K}$ gallons

Supernate Volume: $3 \mathrm{~K}$ gallons

Dränable Interstitial Liquid: OK gallons

Isolation Status

Date Interim Stabilized: 05/31/85

Date Interim Isolated: 06/07/85

\section{Surface Level/Leak Status}

Integrity Category: Asst med Leaker

Date Declared Confirmed or Assumed Leaker: 1978

Leak Volume Estimate: 2,000 Gallons (1989)

FIC Surface Level: 7.60 Inches (12/21/92)

Last Photographed: 05/29/85

Photo Interpretation Comments:

Photographs show a shallow clear liquid surface with patches of light, fllmy material floating in some areas and exposed solids around the tank perimeter. The FIC plummet is contacting solids near the edge of the liquid.

\section{Temperature Status}

Highest temperature during 1992: $63.20^{\circ} \mathrm{F}(07 / 02 / 92)$

Comments: Temperatures are stable.

Drywell Status

Comments: Current drywell profiles were stable and consistent with established baseline profiles.
Tank Description

Type: Single Shell

Constructed: 1944

n-service: $04 / 46$

Capacity: $530 \mathrm{~K}$ gallons

Bottom shape: Dish

Hanford Coordinates:

$45,437^{\circ}$ North

52,852' West

\section{Leak Detection System \\ Surface Level: \\ FIC Riser- R-04 \\ Manual Tape Riser- Yes \\ LOW Riser(s)- None}

Ventilation: Passive 
WHC-EP-0625

This page intentionally left blank. 


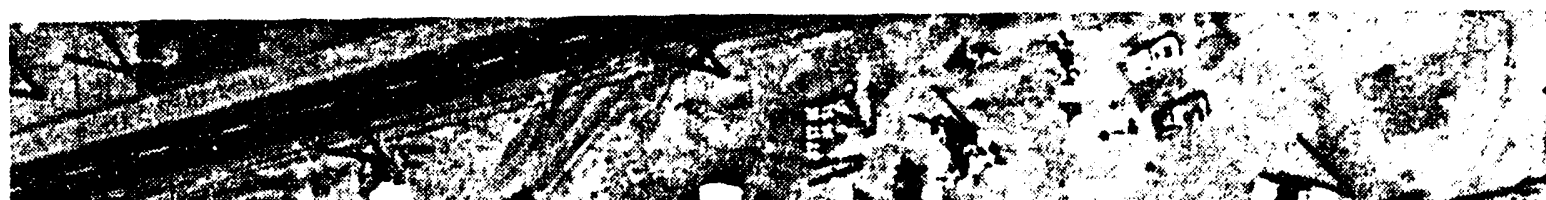

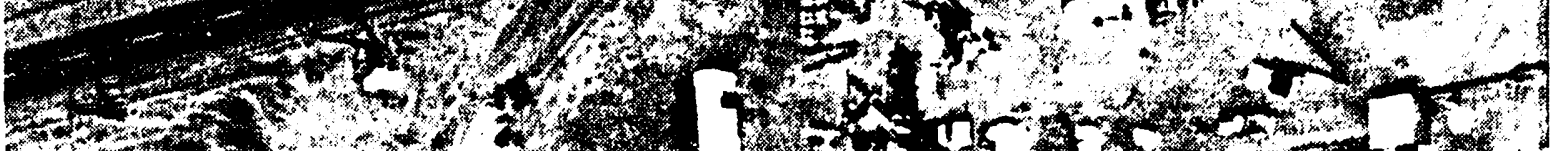

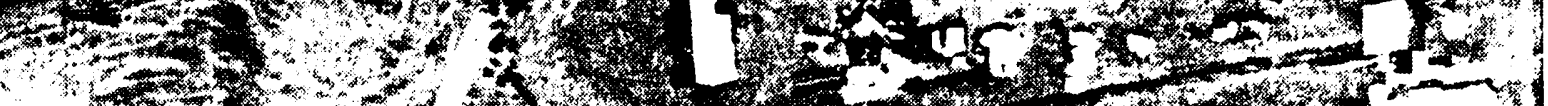

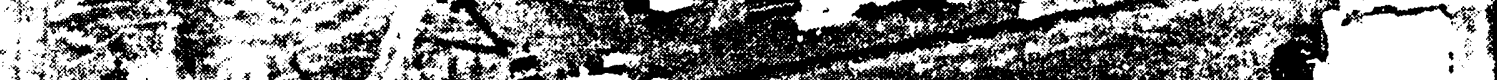

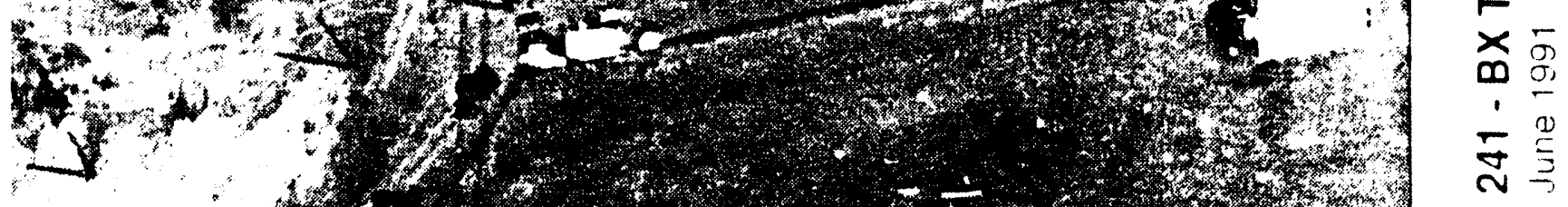

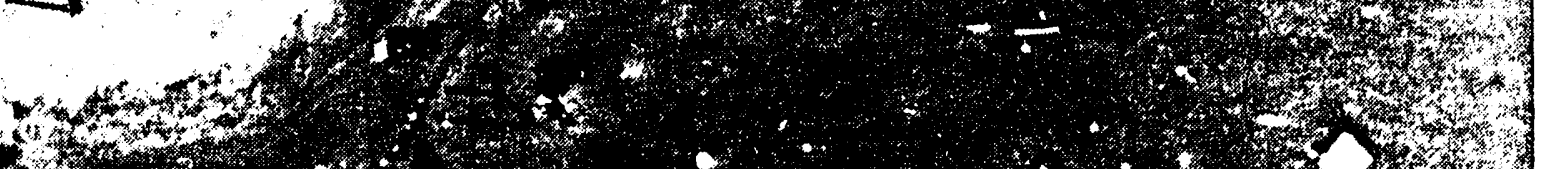

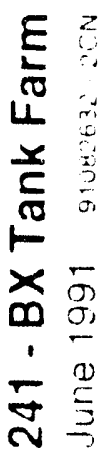

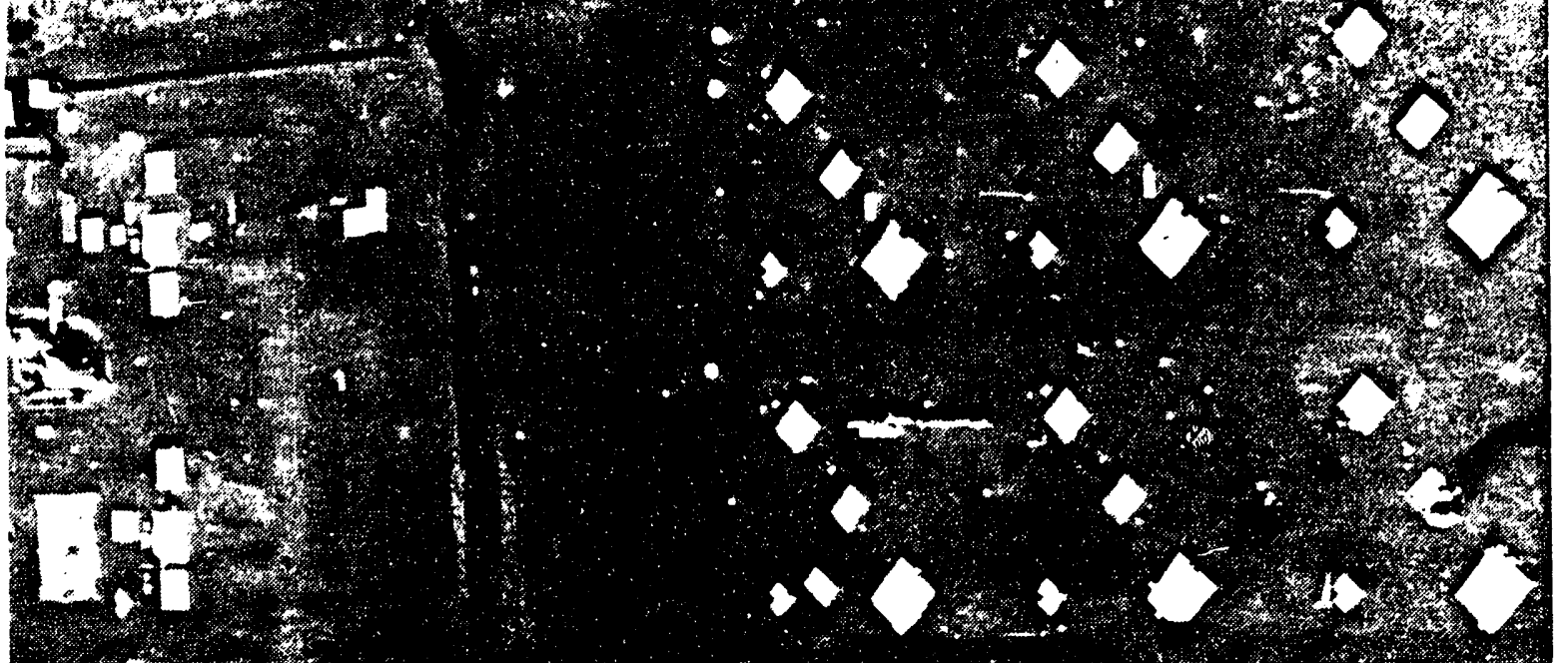

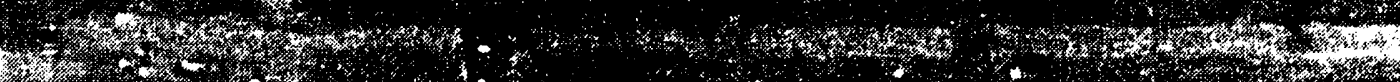

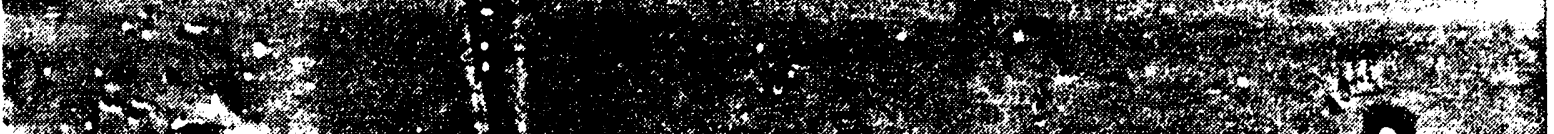

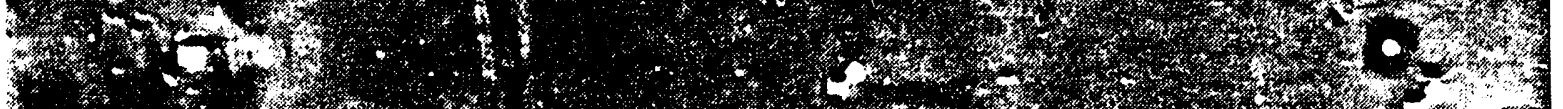

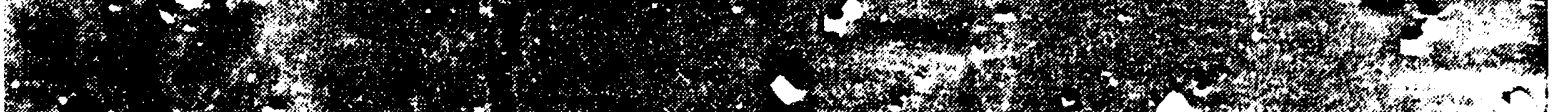

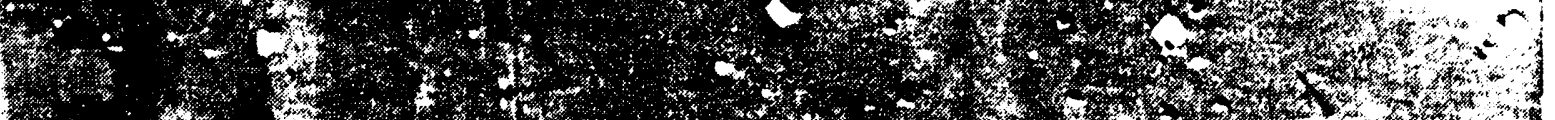

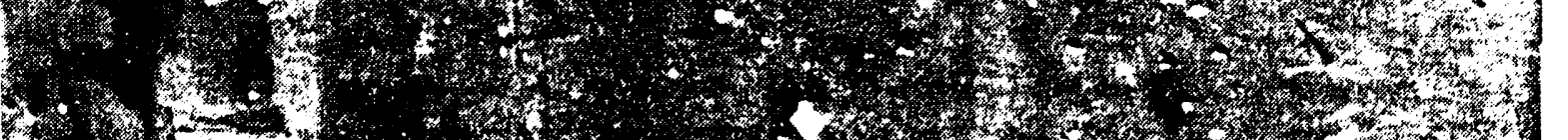

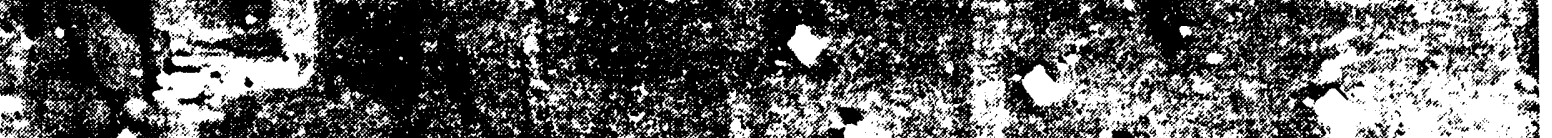

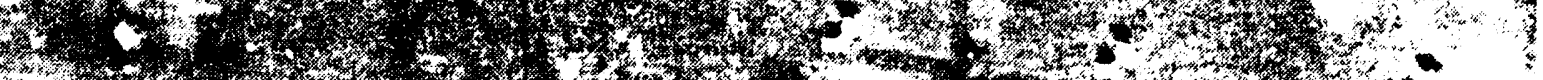

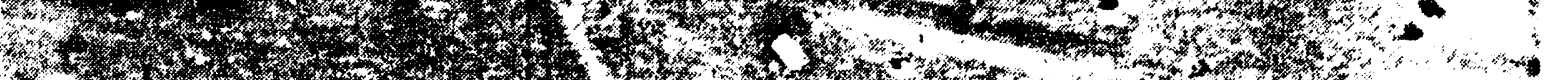
2.7. -

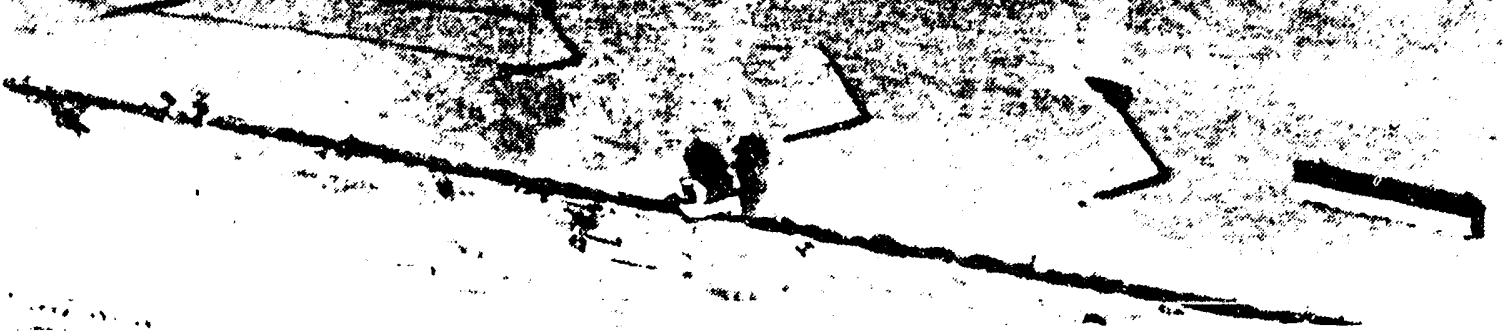

151 
WHC-EP-0625

This page intentionally left blank. 


\section{Tank 241-BX-101}

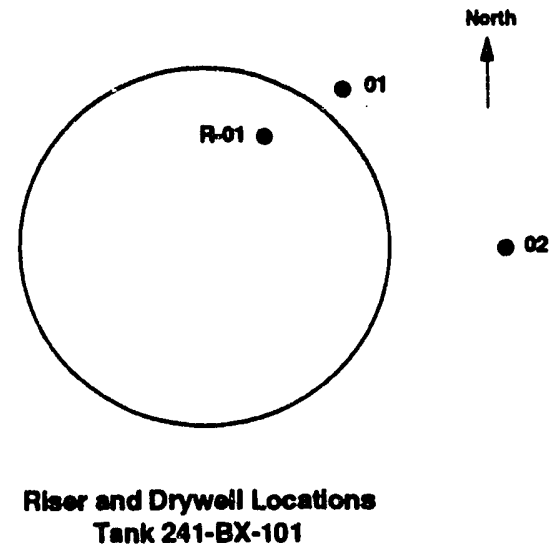

Number of External Drywells: 2

Number of Lateral Wells: None
Tank Description

Type: Single Shell

Constructed: 1947

In-service: $01 / 17 / 48$

Out of Service: 1972

Diameter: $7^{\circ}$

Usable Depth: 16'

Capacity: 530K gallons

Bottom shape: Dish

Hanford Coordinates:

$45,400^{\prime}$ North

$53,250^{\circ}$ West

Ventilation: Passive

\section{Leak Detection System}

Surface Level:

FIC Riser- None

Manual Tape Riser- R-01

LOW Riser(s)- None

\section{Tank Status}

\section{Contents}

Type: Non-Complexed Waste

Total Waste: $43 \mathrm{~K}$ gallons

Supernate Volume: $1 \mathrm{~K}$ gallons

Drainable Interstitial Liquid: OK gallons

Isolation Status

Date Interim Stabilized: 09/78

Date Interim Isolated: 05/01/81

\section{Surface Level/Leak Status}

Integrity Category: Assumed Leaker

Date Declared Confirmed or Assumed Leaker: 1972

Leak Volume Estimate: $7.8 \mathrm{~K}$ gallons $=1 / 19$ th of $150 \mathrm{~K}$ gallons

Manual Tape Surface Level: 10.00 Inches (10/05/92)

Last Photographed: 11/24/88

Photo Interpretation Comments:

Photographs from 08/21/86 and 10/28/86 show exposed sludge around the perimeter of the tank and more surface liquid toward the center of the tank than previous photographs. The manual tape plummet now has a small pool of liquid beneath it that is not connected to the larger center pool. Discarded tapes are near the plummet.

Temperature Status

Highest temperature during 1992: $75.50^{\circ} \mathrm{F}(11 / 06 / 92)$

Comments: Temperatures are stable.

Dryweil Status

Comments: Current drywell profiles were stable and consistent with established baseline proflles. 
WHC-EP-0625

This page intentionally left blank. 


\section{Tank 241-BX-102}

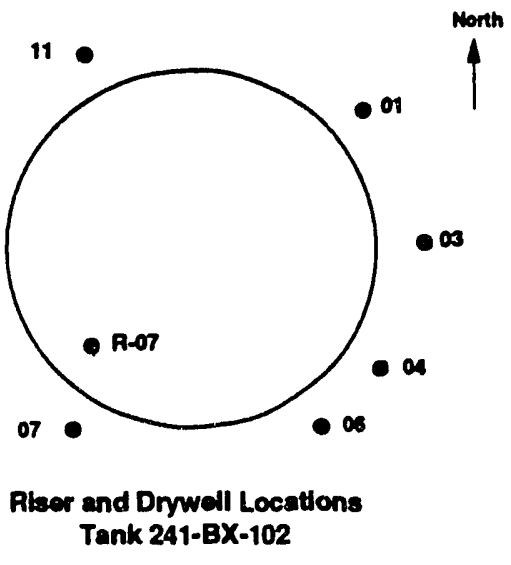

Number of External Drywells: 6

Number of Lateral Wells: None

\section{Tank Status}

Watch List: Ferrocyanide

Contents

Type: Non-Complexed Waste

Total Waste: $96 \mathrm{~K}$ gallons

Supernate Volume: OK gallons

Drainable Interstitial Liquid: $4 \mathrm{~K}$ gallons

Isolation Status

Date Interim Stabilizzed: 11/78

Date Interim Isolated: $10 / 80$

Surface Level/Leak Status

Integrity Category: Assumed Leaker

Date Declared Confirmed or Assumed Leaker: 1971

Leak Volume Estimate: 70,000 Gallons (1986)

Manual Tape Surface Level: 28.25 Inches (10/05/92)

Last Photographed: 09/18/85

Photo Interpretation Comments: See attached photo.

Temperature Status

Highest temperature during 1992: $71^{\circ} \mathrm{F}(09 / 27 / 92)$

Comments: Temperatures are stable.

Drywell Status

Comments: Current drywell profiles were stable and consistent with established baseline profiles.
Tank Description

Type: Single Shell

Constructed: 1947

In-service: 06/10/48

Out of Service: $05 / 70$

Capacity: $530 \mathrm{~K}$ gallons

Bottom shape: Dish

Hanford Coordinates: $45.500^{\circ}$ North 53,250 ' West

Ventilation: Passive

\section{Leak Detection System}

Surface Level:

FIC Riser- None

Manual Tape Riser- R-07

LOW Riser(s)- None
Usable Depth: 16' 
WHC-EP-0625

This page intentionally left blank. 
WHC-EP-0625

\section{Tank: 241-BX-102}

\section{November 1978}

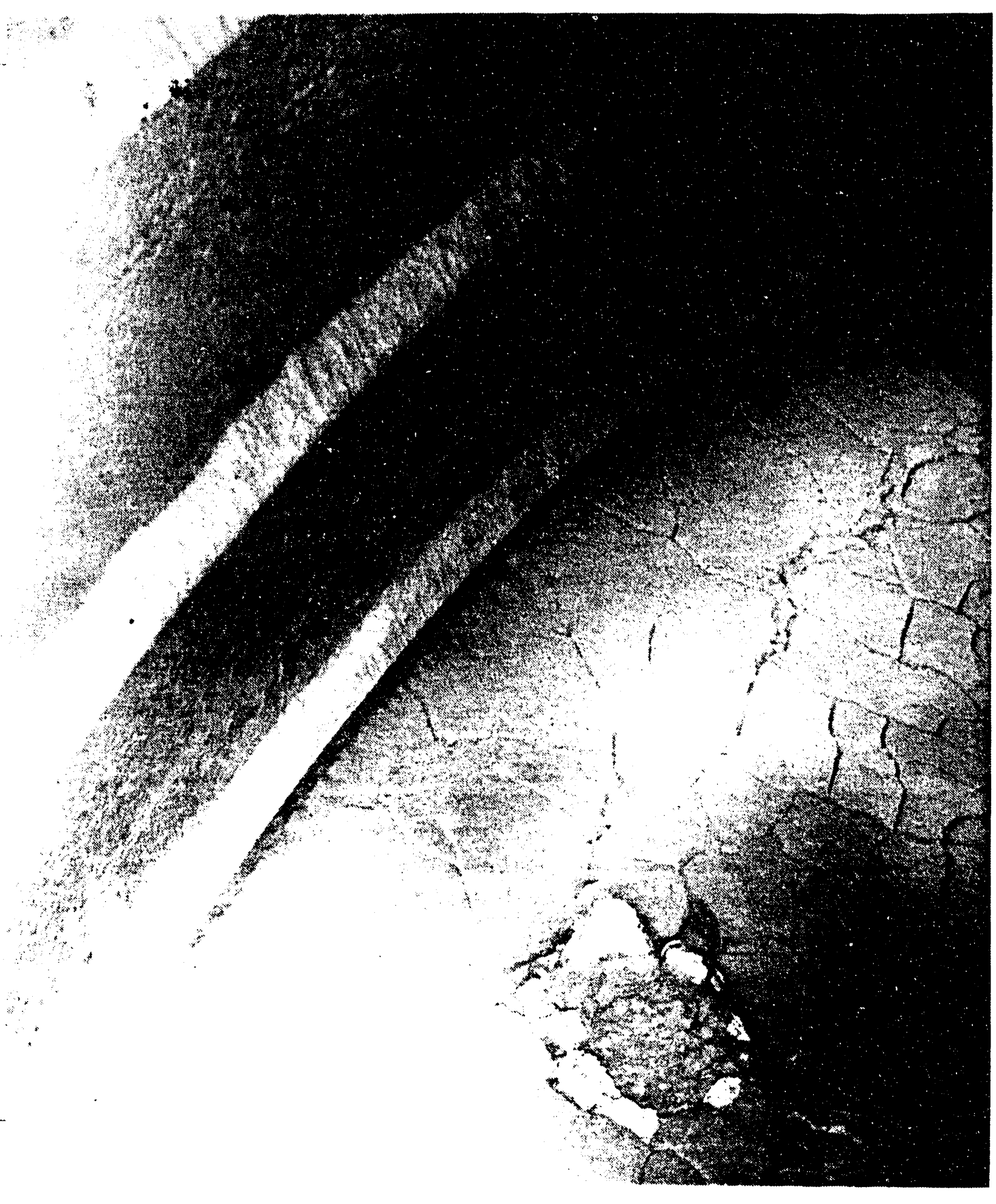


WHC-EP-0625

This page intentionally left blank. 


\section{Tank 241-BX-103}

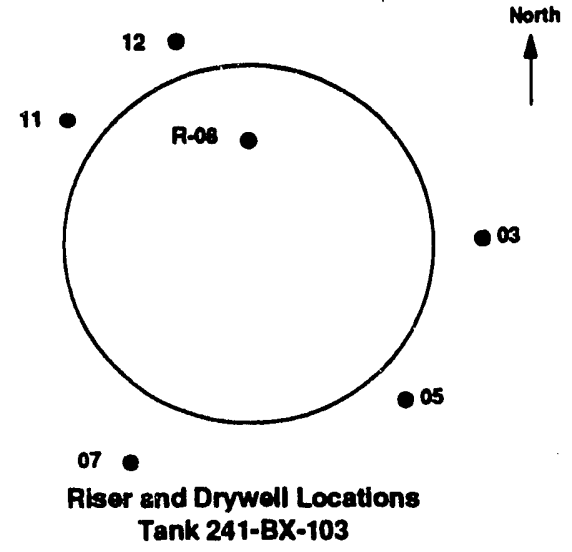

Number of External Drywells: 5

Number of Lateral Wells: None

\section{Tank Status}

\section{Contents}

Type: Non-Complexed Waste

Total Waste: $66 \mathrm{~K}$ gallons

Supernate Volume: $4 \mathrm{~K}$ gallons

Drainable Interstitial Liquid: OK gallons

Isolation Status

Date Interim Stabilized: $11 / 29 / 83$

Date Interim Isolated: 10/30/85

\section{Surface Level/Leak Status}

Integrity Category: Sound

FIC Surface Level: 20.00 Inches (12/21/92)

Last Photographed: 10/31/86

Photo Interpretation Comments:

Photograph comparisons continue to show more surface liquid and less exposed sludge around the tank perimeter. The FIC plummet near the edge of the tank is contacting a clear liquid surface.

\section{Temperature Status}

Highest temperature during 1992:

Drywell Status

Comments: Current drywell profiles were stable and consistent with established

baseline profiles.
Tank Description

Type: Single Shell

Constructed: 1947

In-service: $09 / 30 / 48$

Dlameter: $\mathbf{7 5}^{\circ}$

Usable Depth: 16'

Bottom shape: Dish

Hanford Coordinates:

45,600' North

$53,250^{\circ}$ West

Ventilation: Passive

\section{Leak Detection System \\ Surface Level: \\ FIC Riser- R-08 \\ Manual Tape Riser- None \\ LOW Riser(s)- None}

Capacity: $530 \mathrm{~K}$ gallons 
WHC-EP-0625

This page intentionally left blank. 


\section{Tank 241-BX-104}

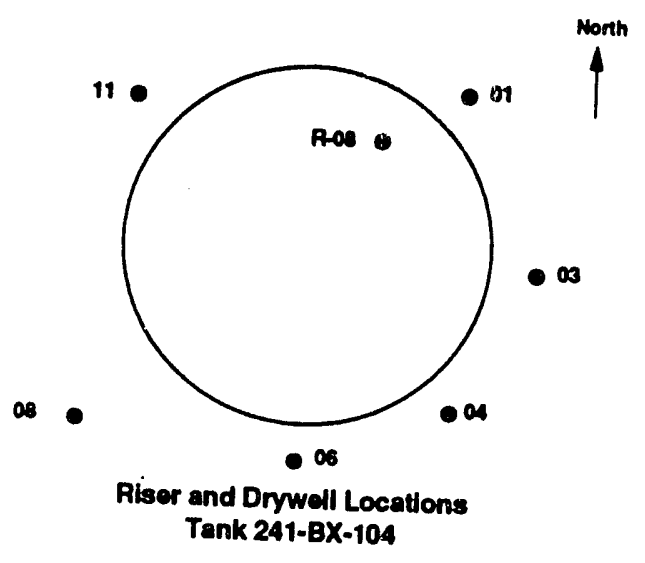

Number of External Drywells: 6 Number of Lateral Wells: None

\section{Tank Status}

Contents

Type: Non-Complexed Waste

Total Waste: $99 \mathrm{~K}$ gallons

Supernate Volume: $3 \mathrm{~K}$ gallons

Drainable Interstitial Liquid: $30 \mathrm{~K}$ gallons

Surface Level/Leak Status

Interim Isolated:

Interim Stabllized: 09/89

Surface Level/Leak Status

Integrity Category: Sound

FIC Surface Level: 32.00 Inches (12/28/92)

Last Photographed: 09/21/89

Photo Interpretation Comments:

Photograph comparisons show more surface liquid and less exposed sludge
around the perimeter of the tank. The FIC plummet is contacting liquid.

Temperature Status

Highest temperature during 1992:

\section{Drywell Status}

Comments: Current drywell profiles were stable and consistent with established
baseline profiles.
Tank Description

Type: Single Shell

Constructed: 1947

In-service: 1949

Out of Service: 1980

Diameter: $7^{\circ}$

Usable Depth: 16'

Capacity: $530 \mathrm{~K}$ gallons

Bottom shape: Dish

Hanford Coordinates:

45, 400' North

53,350' West

Ventilation: Passive

Leak Detection System

Surface Level:

FIC Riser- R-08

Manual Tape Riser- None

LOW Riser(s)- None 
WHC-EP-0625

This page intentionally left blank. 


\section{Tank 241-BX-105}

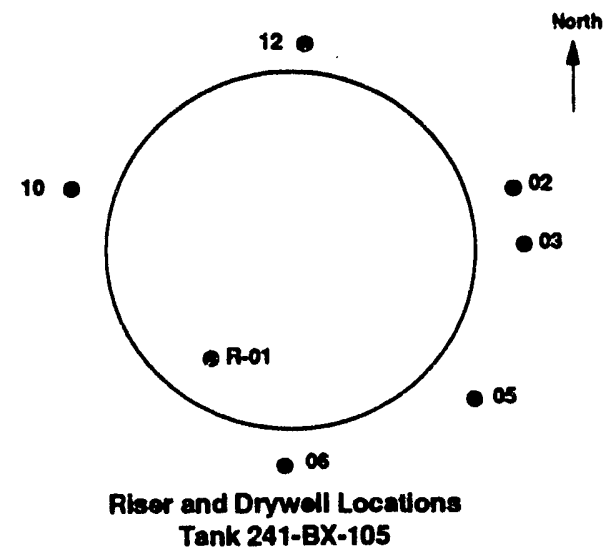

Number of External Drywells: 6

Number of Lateral Wells: None

\section{Tank Status}

\section{Contents}

Type: Non-Complexed Waste

Total Waste: $51 \mathrm{~K}$ gallons

Supernate Volume: $5 \mathrm{~K}$ gallons

Drainable Interstitial Liquid: $6 \mathrm{~K}$ gallons

Isolation Status

Date Interim Stabilized: $03 / 81$

Date Interim Isolated: $09 / 25 / 86$

\section{Surface Level/Leak Status}

Integrity Category: Sound

FIC Surface Level: 25.80 Inches (12/21/92)

Surface Level Comments: Intrusion Mode

Last Photographed: 10/23/86

Photo Interpretation Comments:

Photographs from 03/24/82 show a slightly larger clear liquid sure ae and exposed solids around the perimeter of the tank. Photographs fror:i 10/20/86 (taken on completion of pumping) show some liquid toward the tank center. The FIC plummet is contacting a piecc of pipe protruding from the solids directly beneath it and also discarded tapes.

\section{Temperature Status}

Highest temperature during 1992: $71^{\circ} \mathrm{F}(01 / 02 / 92)$

Comments: Temperatures are stable.

\section{Drywell Status}

Comments: Current drywell profiles were stable and consistent with established baseline profiles.
Tank Description

Type: Single Shell

Constructed: 1947

In-service: 1949

Diameter: $75^{\circ}$

Usable Depth: $16^{\prime}$

Capacity: $530 \mathrm{~K}$ gailons

Bottom shape: Dish

Hanford Coordinates:

45.500' North

53,350' West

Ventilation: Passive

Leak Detection System

Surface Level:

FIC Riser- R-0 1

Manual Tape Riser- None

LOW Riser(s)- None 
WHC-EP-0625

This page intentionally left blank. 


\section{Tank 241-BX-106}

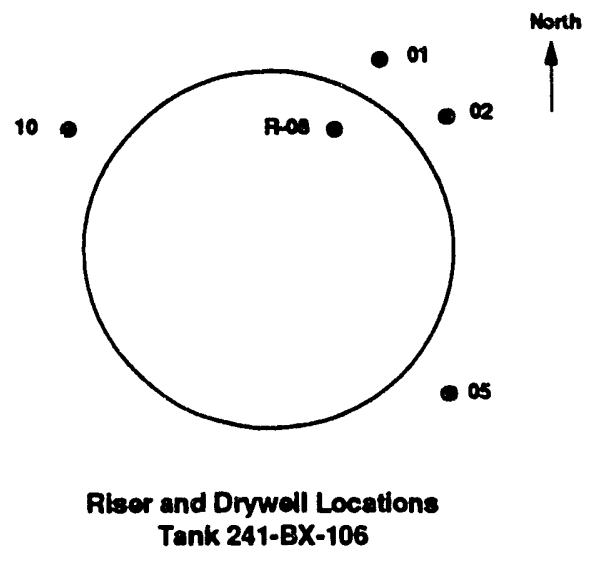

Number of External Drywells: 4 Number of Lateral Wells: None

\section{Tank Status}

Watch List: Ferrocyanide

Contents

Type: Non-Complexed Waste

Total Waste: $46 \mathrm{~K}$ gallons

Supernate Volume: $15 \mathrm{~K}$ gallons

Drainable Interstitial Liquid: OK gallons

Surface Level/Leak Status

Integrity Category: Sound

FIC Surface Level: 12.30 Inches (12/28/92)

Last Photographed: 05/19/88

Photo Interpretation Comments: Siee attached photo.

Temperature Status

Highest temperature during 1992: $69.9^{\circ} \mathrm{F}(10 / 18 / 92)$

Comments: Temperatures are stable.

Drywell Status

Comments: Current drywell proflles were stable and consistent with established baseline profiles.
Tank Description

Type: Single Shell

Constructed: 1947

In-service: 1949

Diameter: $75^{\circ}$

Usable Depth: 16'

Capacity: $530 \mathrm{~K}$ gallons

Bottom shape: Dish

Hanford Coordinates:

$45,600^{\circ}$ North

53,350' West

Ventilation: Passive

\section{Leak Detection System}

Surface Level:

FIC Riser- R-08

Manual Tape Riser- None

LOW Riser(s)- None 


\section{WHC-EP-0625}

This page intentionally left blank. 


\title{
Tank: 241-BX-106
}

\author{
May 1988
}

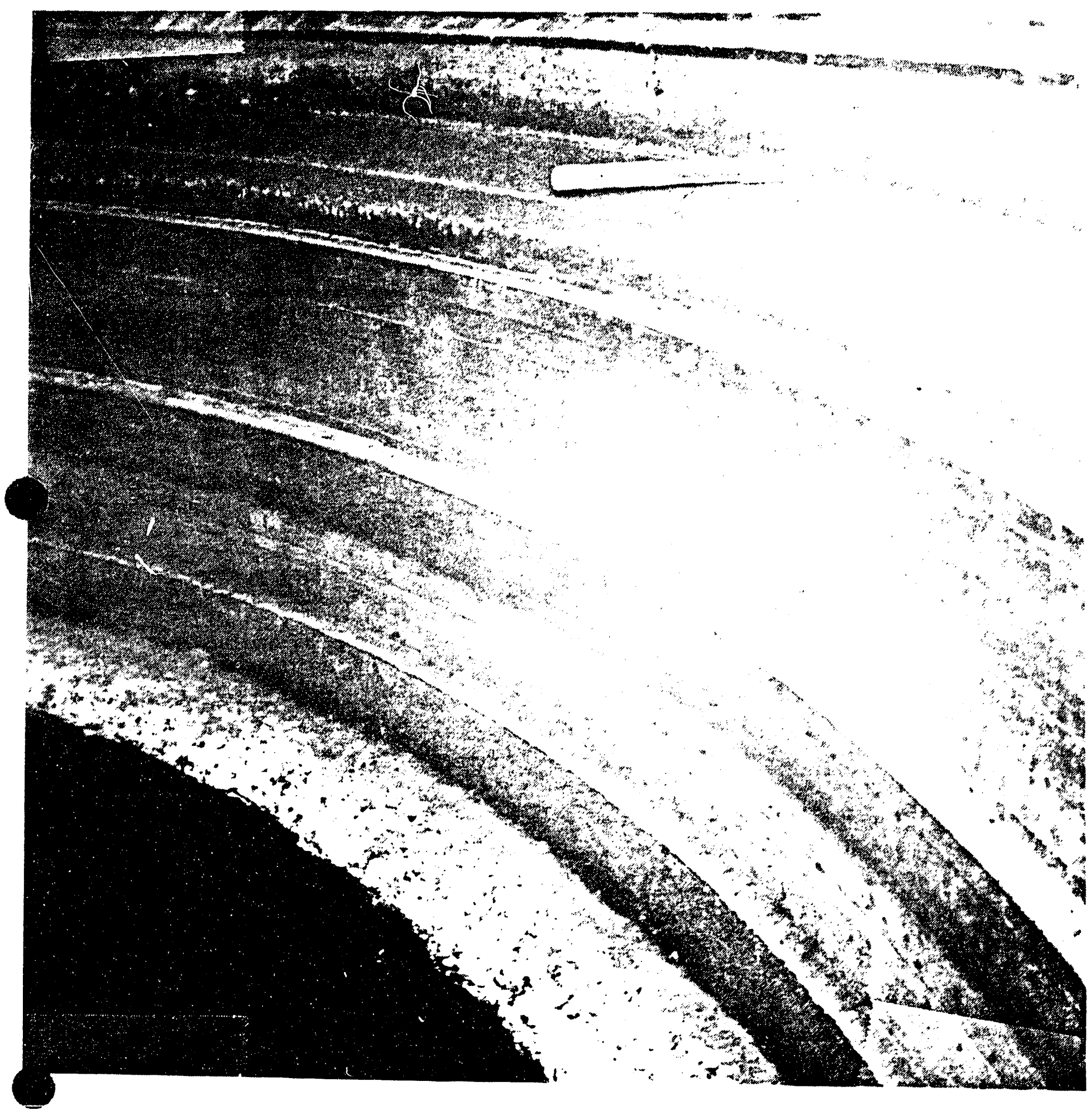




\section{WHC-EP-0625}

This page intentionally left blank. 


\section{Tank 241-BX-107}

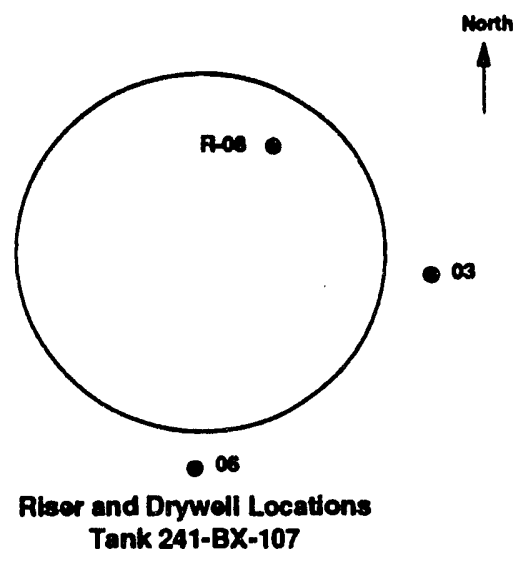

Number of External Dryweils: 2

Number of Lateral Wells: None

\section{Tank Status \\ Contents}

Type: Non-Complexed Waste

Total Waste: $345 \mathrm{~K}$ gallons

Supernate Volume: IK gallons

Drainable Interstitial Liquid: $29 \mathrm{~K}$ gallons

\section{Isolation Status}

Date Interim Isolated:

Date Interim Stabilized: 09/90

\section{Surface Level/Leak Status}

Integrity Category: Sound

FIC Surface Level: 121.00 Inches (12/28/92)

Last Photographed: 09/11/90

Photo Interpretation Comments:

Photographs show exposed solids and a slightly larger area of liquid than previous photographs. The surface liquid is partially covered with a film containing trapped gas bubbles in what appears to be organic. The FIC plummet is contacting liquid.

Temperature Status

Highest temperature during 1992:

\section{Drywell Status}

Comments: Current drywell profiles were stable and consistent with established baseline profiles.
Tank Description

Type: Single Shell

Constructed: 1947

In-service: 09/48

Diameter: $7^{\circ}$

Usable Depth: 16'

Capacity: $530 \mathrm{~K}$ gallons

Bottom shape: Dish

Hanford Coordinates:

$45,400^{\prime}$ North

53,450' West

Ventilation: Passive

\section{Leak Detection System \\ Surface Level: \\ FIC Riser- R-08 \\ Manual Tape Riser- None \\ LOW Riser(s)- None}


WHC-EP-0625

This page intentionally left blank. 


\section{Tank 241-BX-108}

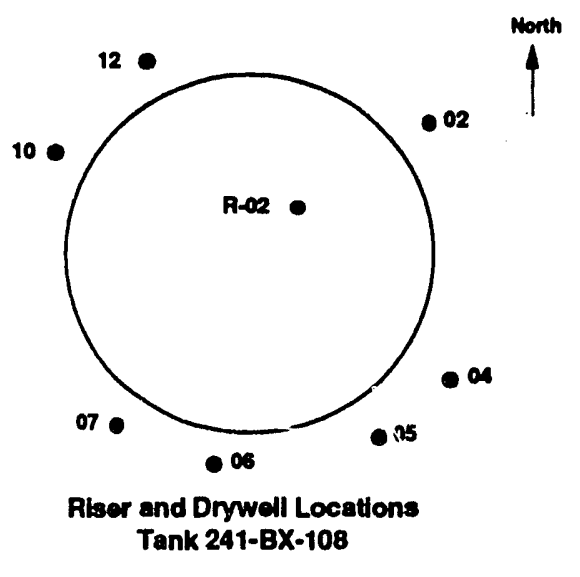

Number of External Drywells: 7 Number of Lateral Wells: None

\section{Tank Status}

Contents

Type: Non-Complexed Waste

Total Waste: $26 \mathrm{~K}$ gallons

Supernate Volume: OK gallons

Drainable i.iterstitial Liquid: $1 \mathrm{~K}$ gallons

Isolation Status

Date Interim Stabilized: $07 / 79$

Date Interm Isolated: 06/81

Surface Level/Leak Status

Integrity Category: Assumed Leaker

Date Declared Confirmed or Assumed Leaker: 1974

Leak Volume Estimate: 2,500 Gallons (1986)

Manual Tape Surface Level: 4.50 Inches (10/05/92)

Last Photographed: $10 / 23 / 86$

Photo Interpretation Comments:

Photographs from 11/09/82 show a dry sludge surface and a small pool of liquid where a pump was removed. The manual tape plummet is contacting solids in a small depression. Photographs from 10/23/86 show little change. The small

Temperature Status liquid pool in previous photographs is no longer visible.

Highest temperature during 1992: $61.30^{\circ} \mathrm{F}(07 / 03 / 92)$

Comments: Temperatures are stable.

Drywell Status

Comments: Current drywell profiles were stable and consistent with established baseline profiles. 
WHC-EP-0625

This page intentionally left blank. 


\section{Tank 241-BX-109}

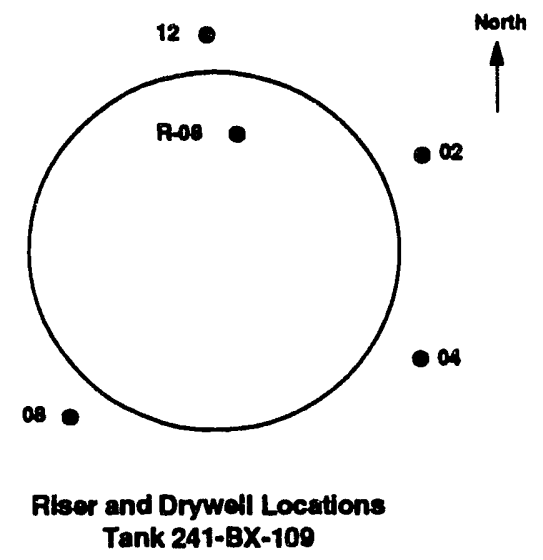

Number of External Drywells: 4 Number of Lateral Wells: None

\section{Tank Status}

\section{Contents}

Type: Non-Complexed Waste

Total Waste: 193K gallons

Supernate Volume: OK gallons

Dratnable Interstitial Liquid: $13 \mathrm{~K}$ gallons

Isolation Status

Date Interim Isolated:

Date Interim Stabilized: 09/90

\section{Surface Level/Leak Status}

Integrity Category: Sound

FIC Surface Level: 65.30 Inches (12/21/92)

Surface Level Comments: Intrusion Mode

Last Photographed: 09/11/90

Photo Interpretation Comments:

Photographs taken on completion of supernatant pumping show a pock-marked surface of sludge with puddles and rivulets of liquid. The FIC piummet is contacting a small puddle and discarded tapes.

\section{Temperature Status}

Highest temperature during 1992:

\section{Drywell Status}

Comments: Current drywell profiles were stable and consistent with established baseline profiles. 
WHC-EP-0625

This page intentionally left blank. 


\section{Tank 241-BX-110}

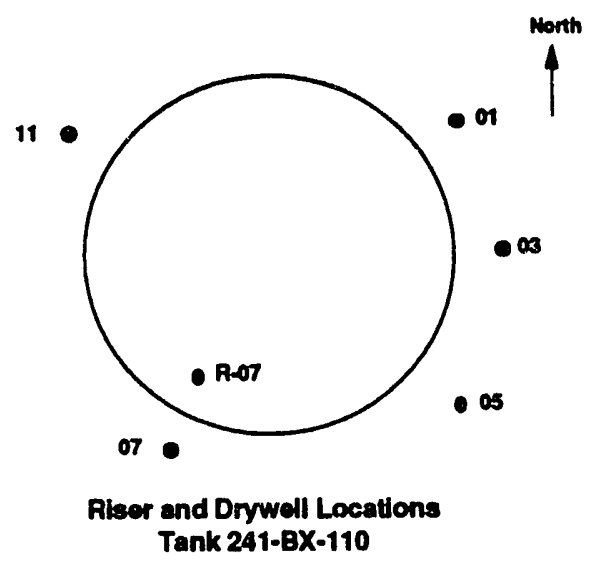

Number of External Drywe:lls: 5

Number of Laterai Wells: None

\section{Tank Status}

\section{Watch List: Ferrocyanide}

Contents

Type: Non-Complexed Waste

Total Waste: $199 \mathrm{~K}$ gallons

Supernate Volume: $1 \mathrm{~K}$ gallons

Drainable Interstitial Liquid: $15 \mathrm{~K}$ gallons

Isolation Status

Date Partially Interim Isolated: 12/15/82

Date Interim Stabulized: 08/22/85

\section{Surface Level/Leak Status}

Integrity Category: Assumed Leaker

Date Declared Confirmed or Assumed Leaker: 1976

Leak Volume Estimate: $7.8 \mathrm{~K}$ gallons $=1 / 19$ th of $150 \mathrm{~K}$ gallons

Manual Tape Surface Level: 68.50 Inches (12/01/92)

Last Photographed: 07/31/85

Photo Interpretation Comments: See attached photo.

Temperature Status

Highest temperature during 1992: $71.2{ }^{\circ} \mathrm{F}(05 / 16 / 92)$

Comments: Temperatures are stable.

\section{Drywell Status}

Comments: Current drywell profiles were stable and consistent with established baseline profiles.
Tank Description

Type: Single Shell

Constructed: 1947

n-service: 1949

eter: 75

: $530 \mathrm{~K}$ gallons

Bottom shape: Dish

Coordinates:

$53.550^{\circ}$ West

\section{Leak Detection System}

Surface Level:

FIC Riser- None

Manual Tape Riser- R-07

LOW Riser(s)- None 
WHC-EP-0625

This page intentionally left blank. 


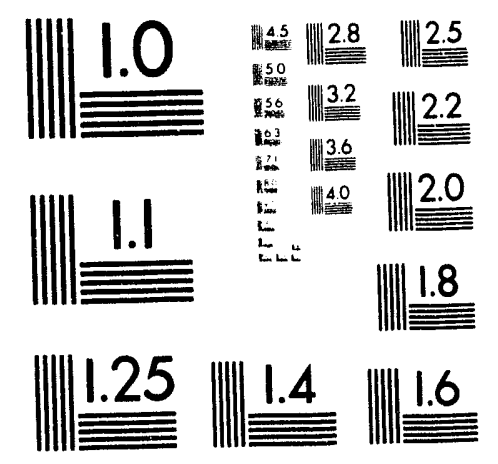



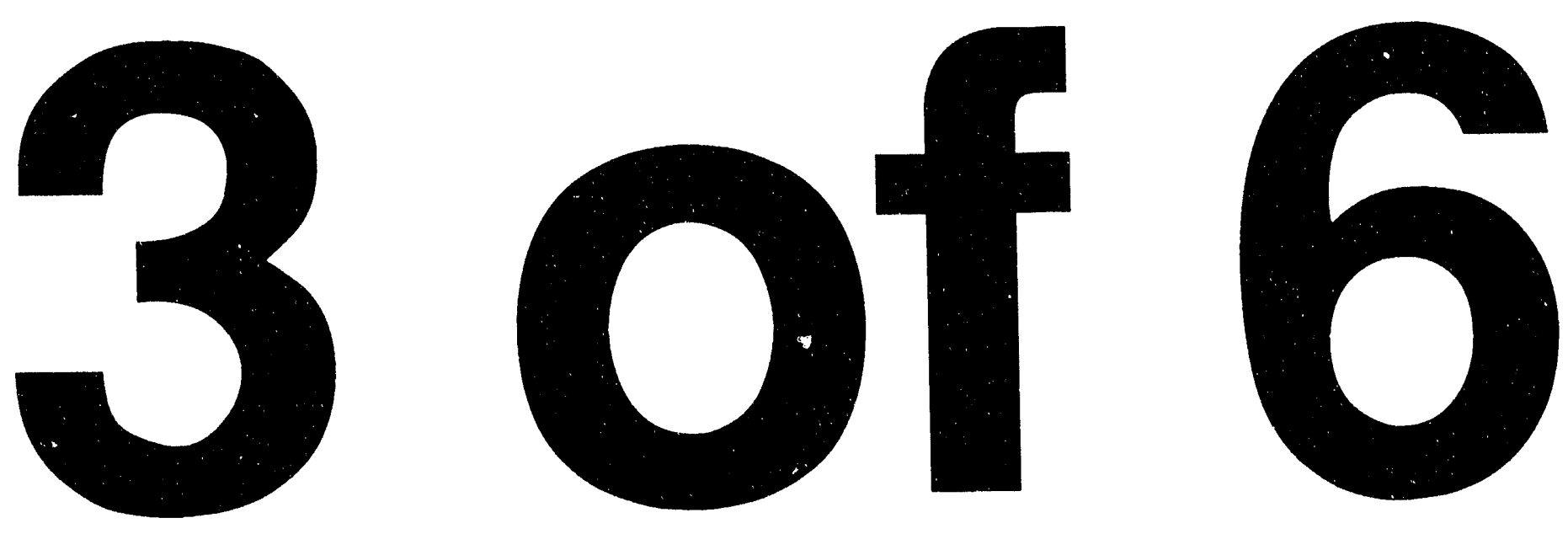


\title{
Tank: 241-BX-110
}

\author{
July 1985
}

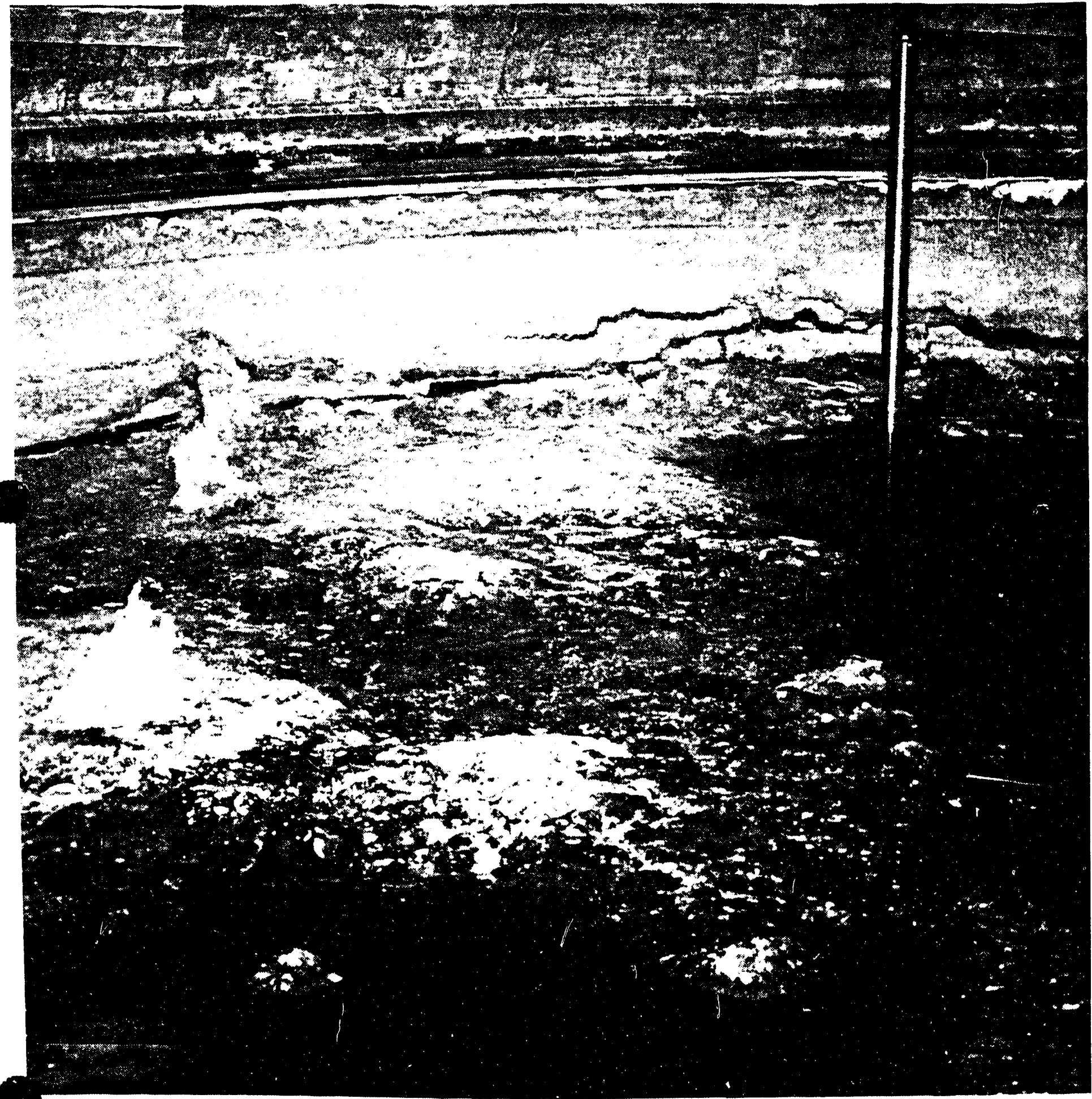


WHC-EP-0625

This page intentionally left blank. 


\section{Tank 241-BX-111}

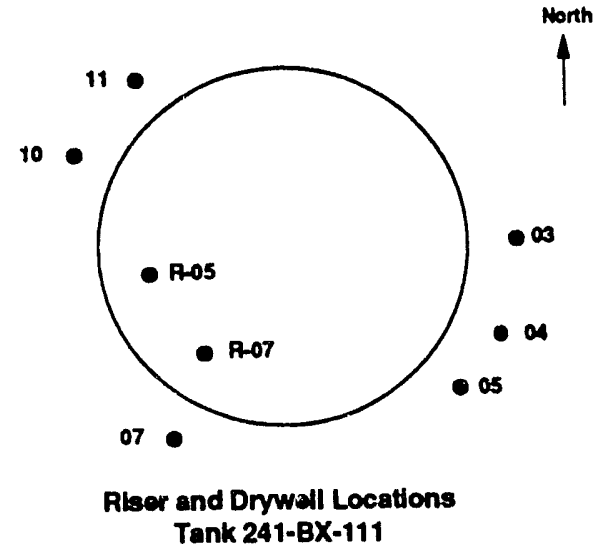

Number of External Drywells: 6

Number of Lateral Wells: None

\section{Tank Status}

Watch List: Ferrocyanide

Contents

Type: Non-Complexed Waste

Total Waste: 230K gallons

Supernate Volume: 19K gallons

Drainable Interstitial Liquid: $50 \mathrm{~K}$ gallons

Isolation Status

Date Partially Interim Isolated: 12/15/82

Surface Level/Leak Status

Integrity Category: Assumed Leaker

Date Declared Confinned or Assumed Leaker: 1984

Leak Volume Estimate: $7.8 \mathrm{~K}$ gallons $=1 / 19$ th of $150 \mathrm{~K}$ gallons

Manual Tape Surface Level: 79.50 Inches (12/28/92)

Last Photographed: 09/18/85

Photo Interpretation Comments: See attached photo.

Temperature Status

Highest temperature during $1992: 71.7^{\circ} \mathrm{F}(11 / 27 / 92)$

Comments: Temperatures are stable.

Drywell Status

Comments: Current drywell profiles were stable and consistent with established baseline profiles.
Tank Description

Type: Single Shell

Constructed: 1947

In-service: 1950

Diameter: $7^{\circ}$

Usable Depth: 16'

Capacity: $530 \mathrm{~K}$ gallons

Hanford Coordinates: $45.500^{\prime}$ North 53.550' West

Ventilation: Passive

\section{Leak Detection System}

Surface Level:

FIC Riser- None

Manual Tape Riser- R-07

LOW Riser(s)- R-05 


\section{WHC-EP-0625}

This page intentionally left blank. 
WHC-EP-0625

\section{Tank: 241-BX-111}

September 1985
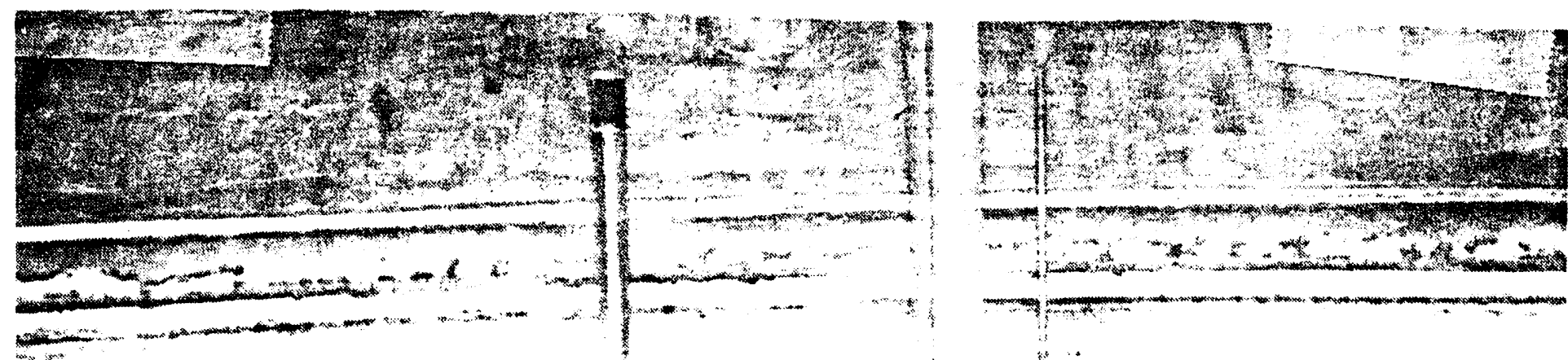

W W

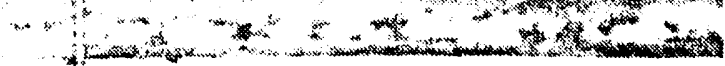
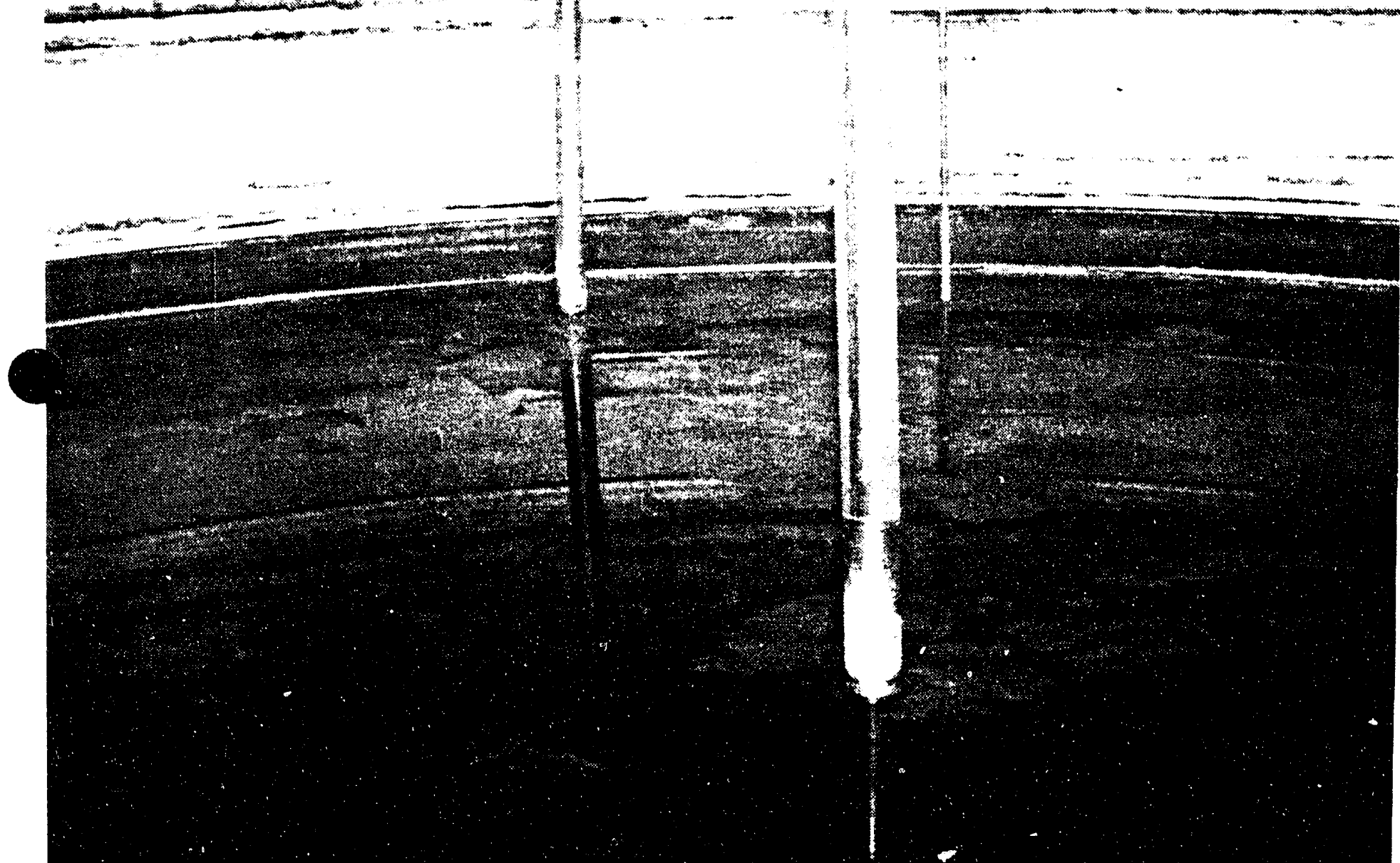

(x)

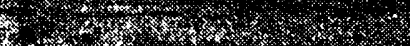

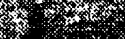

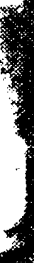
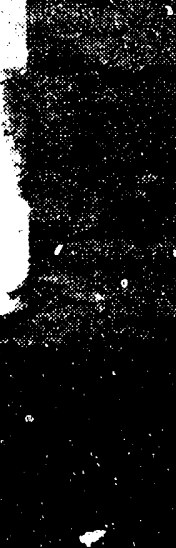

6 
WHC-EP-0625

This page intentionally left blank. 


\section{Tank 241-BX-112}

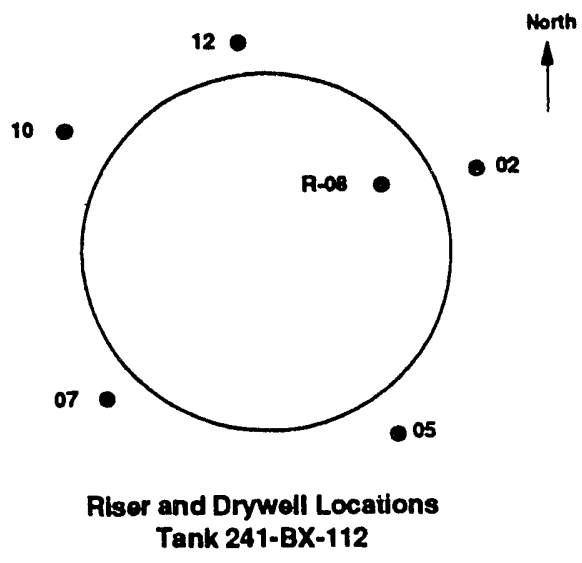

Number of External Drywells: 5

Number of Lateral Wells: None

\section{Tank Status}

\section{Contents}

Type: Non-Complexed Waste

Total Waste: $165 \mathrm{~K}$ gallons

Supernate Volume: $1 \mathrm{~K}$ gallons

Drainable Interstitial Liquid: $7 \mathrm{~K}$ gallons

\section{Isolation Status}

Date Interim Isolated:

Date Interim Stabilized: 09/90

\section{Surface Level/Leak Status}

\section{Integrity Category: Sound}

FIC Surface Level: 55.40 Inches (12/21/92)

Surface Level Comments: Out of service on $12 / 28 / 92$.

Last Photographed: 09/11/90

Photo Interpretation Comments:

Photographs from $5 / 3 / 83$ reveal a very uneven surface of exposed solids and shallow liquid in many areas of the tank. The FIC plummet is contacting a very small, shallow liquid surface near the tank perimeter. There appears to be slightly more liquid than in previous photographs. Photographs from $07 / 16 / 85$ show little

\section{Temperature Status} change from previous photographs.

Highest recent temperature: $69{ }^{\circ} \mathrm{F}(04 / 14 / 91)$

Comments: Temperatures are stable.

\section{Drywell Status}

Comments: Current drywell profiles were stable and consistent with established baseline profiles. 


\section{WHC-EP-0625}

This page intentionally left blank. 




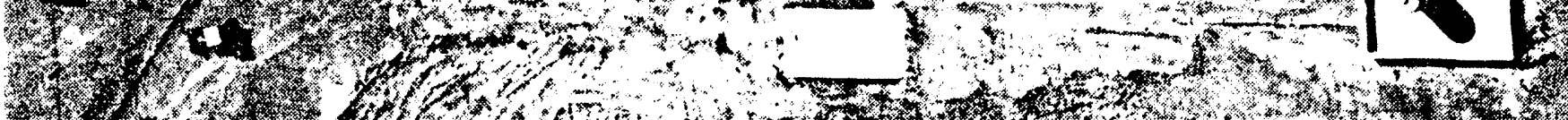

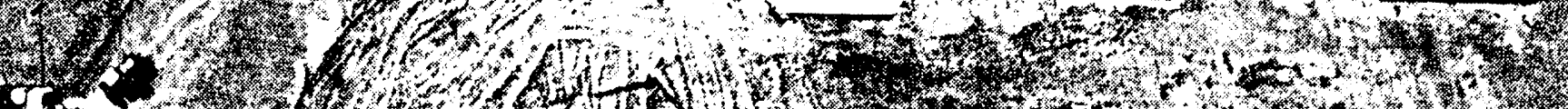

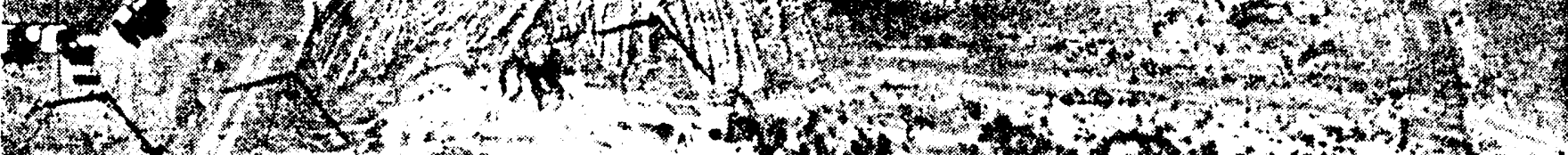

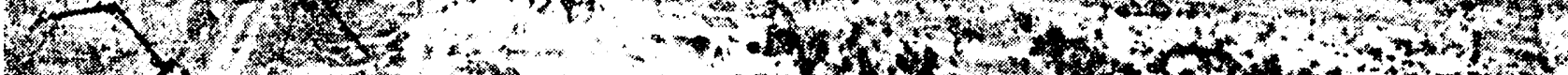

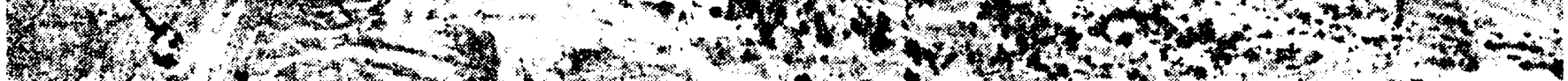

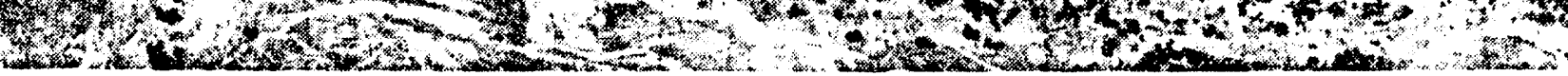




\section{WHC-EP-0625}

This page intentionally left blank. 


\section{Tank 241-BY-101}

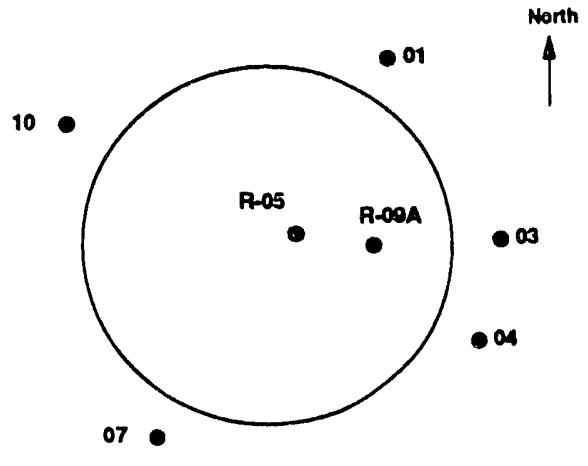

Riser and Drywell Locations Tank 241-BY-101
Tank Description

Type: Single Shell Constructed: 1949 In-service: $01 / 50$ Diameter: 75 ' Usable Depth: 23' Capacity: $758 \mathrm{~K}$ gallons Bottom shape: Dish Hanford Coordinates: 45,898' North $53,247^{\circ}$ West

Ventllation: Passive

\section{Leak Detection System}

Surface Level: FIC Riser- None Manual Tape Riser- R-05 LOW Riser(s)- R-09A

Number of External Drywells: 5

Number of Lateral Wells: None

\section{Tank Status}

Watch List: Ferrocyanide

Contents

Type: Non-Complexed Waste

Total Waste: $387 \mathrm{~K}$ gallons

Supernate Volume: OK gallons

Drainable Interstitial Liquid: $5 \mathrm{~K}$ gallons

Isolation Status

Date Interim Stabilized: 05/22/84

Surface Level/Leak Status

Integrity Category: Sound

Manual Tape Surface Level: 142.25 Inches (12/01/92)

Last Photographed: 09/19/89

Photo Interpretation Comments: See attached photo.

Temperature Status

Highest temperature during 1992: $73^{\circ} \mathrm{F}(08 / 30 / 92)$

Comments: Temperatures are stable.

Drywell Status

Comments: Current drywell profiles were stable and consistent with established baseline profiles. 


\section{WHC-EP-0625}

This page intentionally left blank. 
WHC-EP-0625

Tank: 241-BY-101

September 1989

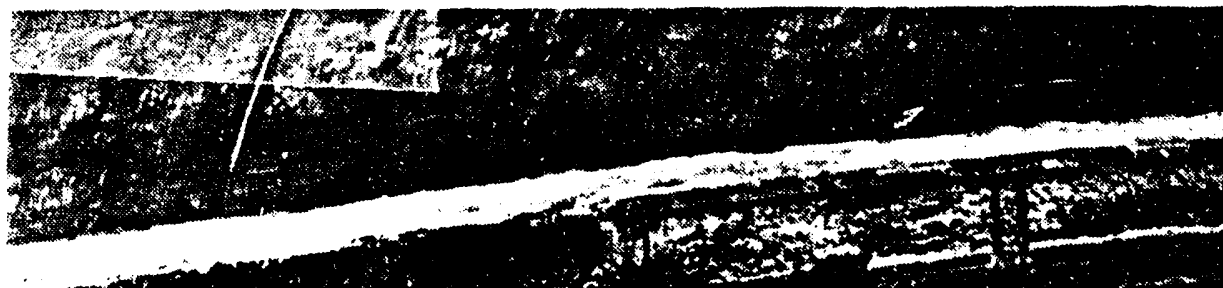

$x_{\infty}^{2}+\infty=$

wotomon

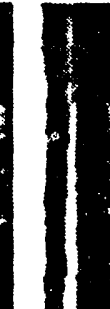

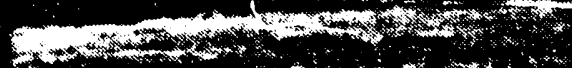

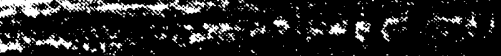

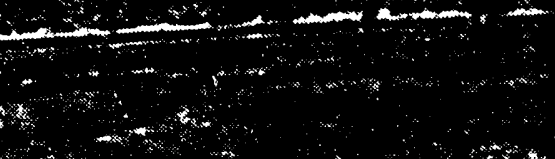

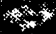

4.

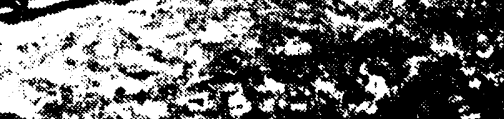

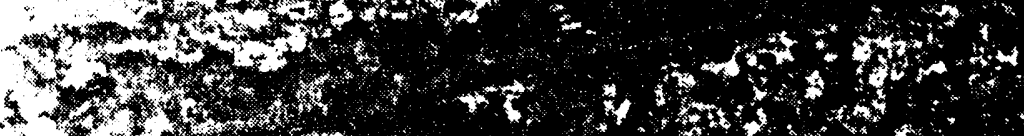

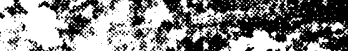

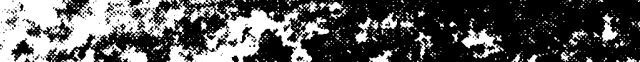

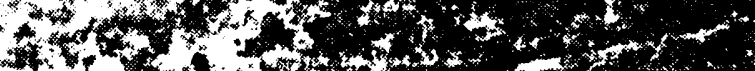

$+x^{2}+x^{2}+4$

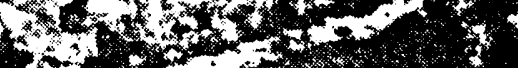

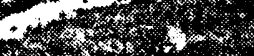

$+23$

$x$

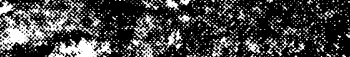

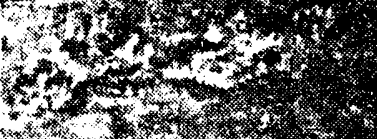

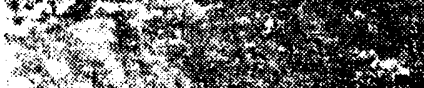

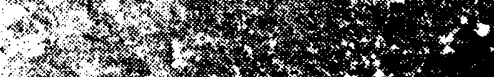

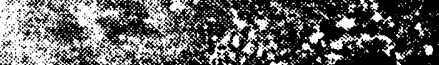

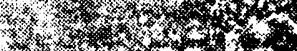

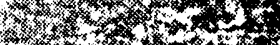

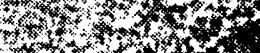

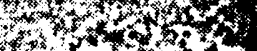

$x_{x}+8 x+2$

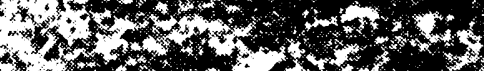

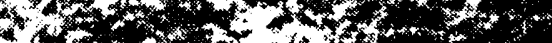

\section{$x+1$}

intas.

$$
3
$$

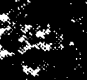

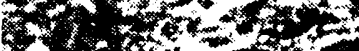

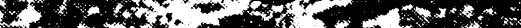

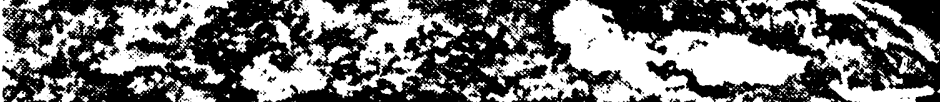

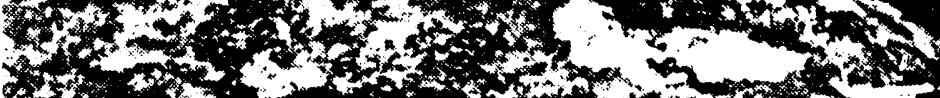

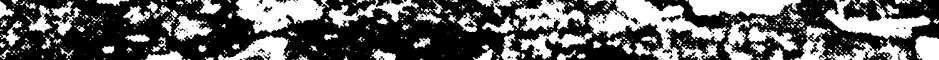

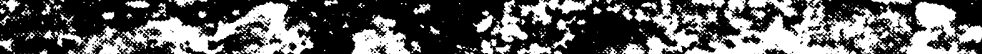

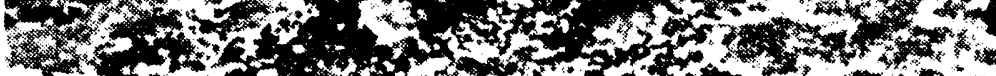

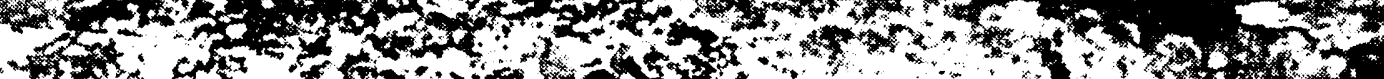

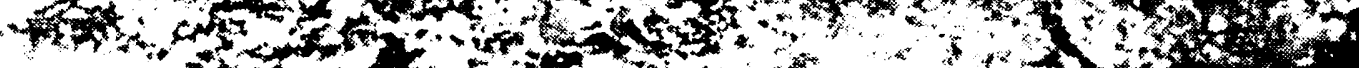

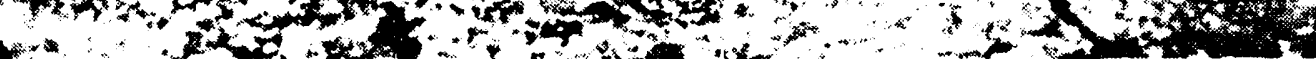

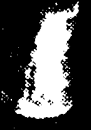


WHC-EP-0625

This page intentionally left blank. 


\section{Tank 241-BY-102}

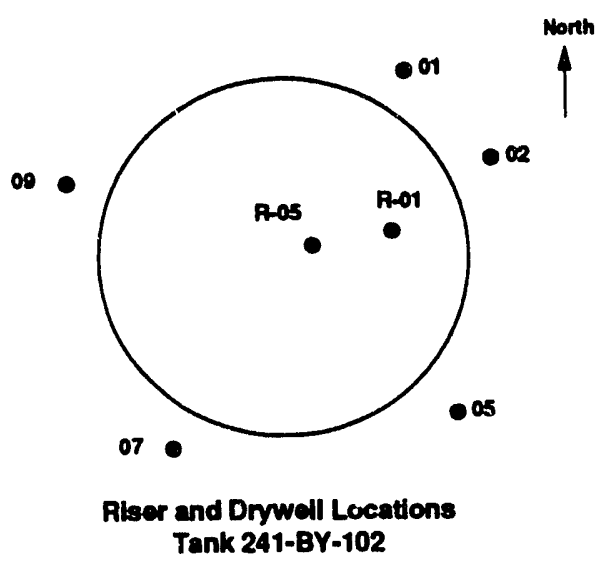

Number of External Drywells: 5

Number of Lateral Wells: None

\section{Tank Status}

\section{Contents}

Type: Non-Complexed Waste

Total Waste: $341 \mathrm{~K}$ gallons

Supernate Volume: OK gallons

Drainable Interstitial Liquid: $41 \mathrm{~K}$ gallons

Isolation Status

Date Partially Interim Isolated: 12/15/82

\section{Surface Level/Leak Status}

Integrity Category: Sound

Manual Tape Surface Level: 78.50 Inches (12/28/92)

Last Photographed: 09/11/87

Photo Interpretation Comments:

Photographs show a wide band of exposed salt cakle around the tank perimeter and a large, deep liquid pool in the center of the tank. The manual tape doughnut

\section{Temperature Status} plummet is contacting a clear liquid surface. Photographs are not current.

Highest recent temperature: $88^{\circ} \mathrm{F}(11 / 02 / 91)$

Comments: Temperatures are stable.

\section{Drywell Status}

Comments: Current drywell profiles were stable and consistent with established baseline profiles.
Tank Description

Type: Single Shell

Constructed: 1949

In-service: $07 / 50$

Diameter: $7^{\circ}$

Usable Depth: 23'

Capacity: $758 \mathrm{~K}$ gallons

Bottom shape: Dish

Hanford Coordinates:

46.000' North

$53,247^{\prime}$ West

Ventilation: Passive

\section{Leak Detection System}

Surface Level:

FIC Riser- None

Manual Tape Riser- R-05

LOW Riser(s)- R-01 
WHC-EP-0625

This page intentionally left blank. 


\section{Tank 241-BY-103}

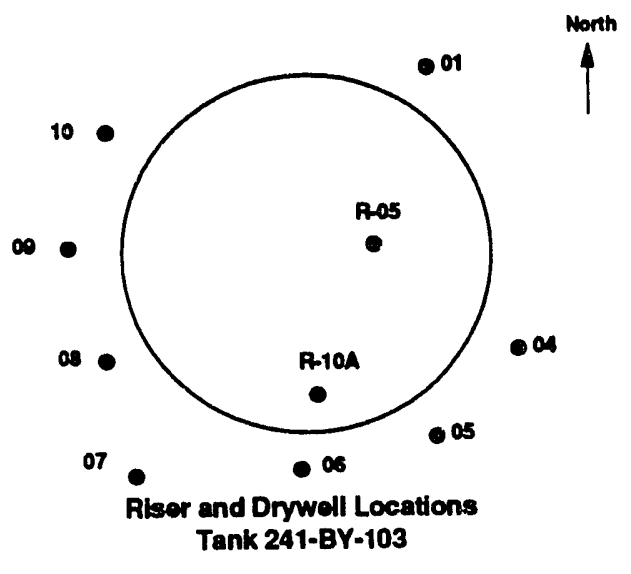

Number of External Drywells: 8 Number of Lateral Wells: None

\section{Tank Status}

Watch List: Ferrocyanide

Contents

Type: Non-Complexed Waste

Total Waste: $400 \mathrm{~K}$ gallons

Supernate Volume: OK gallons

Drainable Interstitial Liquid: $160 \mathrm{~K}$ gallons

Isolation Status

Date Interim Stabilized: 09/19/79

Date Partially Interim Isolated: $12 / 15 / 82$

\section{Surface Level/Leak Status}

Integrity Category: Assumed Leaker

Date Declared Confirmed or Assumed Leaker: 1973

Leak Volume Estimate: < 5,000 Gallons (1983)

Manual Tape Surface Level: 150.25 Inches (12/28/92)

Last Photographed: 09/07/89

Photo Interpretation Comments: See attached photo.

Temperature Status

Highest temperature during 1992: $86.5{ }^{\circ} \mathrm{F}(04 / 11 / 92)$

Comments: Temperatures are stable.

Drywell Status

Comments: Current drywell profiles were stable and consistent with established baseline profiles. 
WHC-EP-0625

This page intentionally left blank. 


\section{Tank: 241-BY-103}

\section{September 1989}

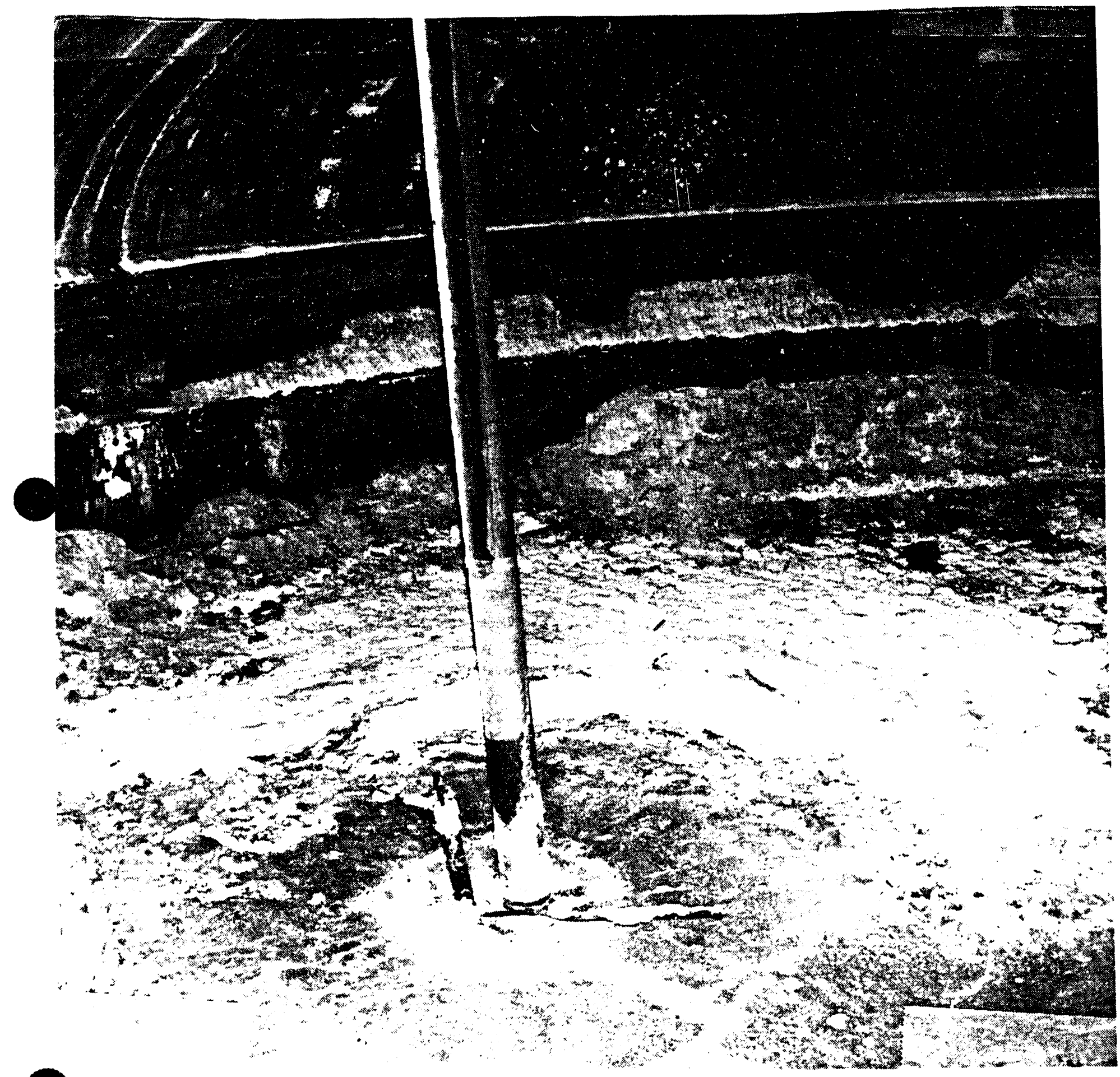




\section{WHC-EP-0625}

This page intentionally left blank. 


\section{Tank 241-BY-104}

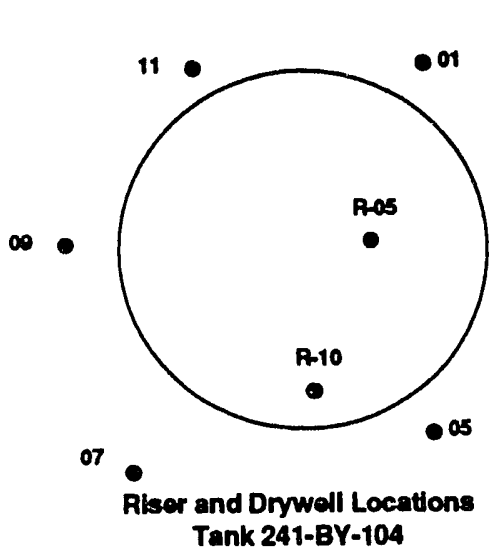

Nonth

Number of External Drywells: 5

Number of Lateral Wells: None

\section{Tank Status}

Watch List: Ferrocyanide

Contents

Type: Non-Complexed Waste

Total Waste: $406 \mathrm{~K}$ gallons

Supernate Volume: OK gallons

Drainable Interstitial Liquid: $18 \mathrm{~K}$ gallons

Isolation Status

Date Interim Stabilized: 01/25/85

\section{Surface Level/Leak Status}

Integrity Category: Sound

Manual Tape Surface Level: 128.25 Inches (12/21/92)

Last Photographed: 04/27/83

Photo Interpretation Comments: See attached photo.

Temperature Status

Highes temperature during 1992: $130.9^{\circ} \mathrm{F}(04 / 25 / 92)$

Comments: Temperatures are stable.

\section{Drywell Status}

Comments: Current drywell profiles were stable and consistent with established baseline profiles.
Tank Description

Type: Single Shell

Constructed: 1949

In-service: $02 / 51$

Diameter: 75

Depth: $23^{\circ}$

Capacity: 758K gallons

Bottom shape: Dish

Hanford Coordinates: $45,898^{\prime}$ North 53,349' West

Ventilation: Passive

Surface Level:

FIC Riser- None

Manual Tape Riser- R-05

LOW Riser(s)- R-10C

\section{Leak Detection System}


WH.C-EP-0625

This page intentionally left blank. 
WHC-EP-0625

\section{Tank: 241-BY-104}

February 1981




WHC-EP-0625

This page intentionally left blank. 


\section{Tank 241-BY-105}

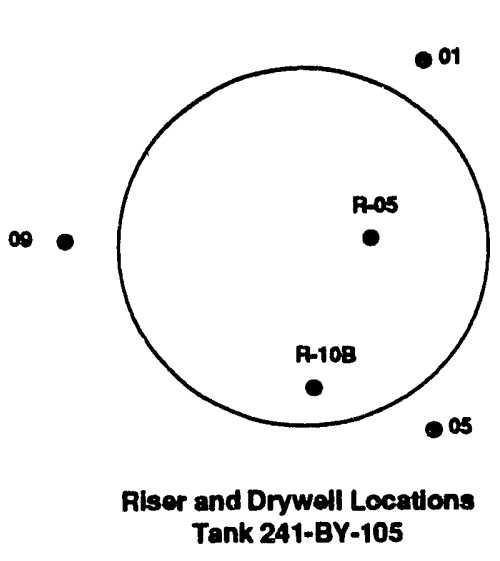

North

Number of External Drywells: 3

Number of Lateral Wells: None

\section{Tank Status}

Watch List: Ferrocyanide

Contents

Type: Non-Complexed Waste

Total Waste: $503 \mathrm{~K}$ gallons

Supernate Volume: OK gallons

Drainabie Interstitial Liquid: $192 \mathrm{~K}$ gallons

Isolation Status

Date Partially Interim Isolated: $12 / 15 / 82$

\section{Surface Level/Leak Status}

Integrity Category: Assumed Leaker

Date Declared Confirmed or Assumed Leaker: 1984

Leak Volume Estimate: $7.8 \mathrm{~K}$ gallons $=1 / 19$ th of $150 \mathrm{~K}$ gallons

Manual Tape Surface Level: 176.00 Inches (12/28/92)

Last Photographed: $07 / 11 / 86$

Photo Interpretation Comments: See attached photo.

Temperature Status

Highest temperature during 1992: $115^{\circ} \mathrm{F}(08 / 15 / 92)$

Comments: Temperatures are stable.

Drywell Status

Comments: Current drywell profiles were stable and consistent with established baseline profiles. 
WHC-EP-0625

This page intentionally left blank. 
WHC-EP-0625

\section{Tank: 241-BY-105}

July 1986

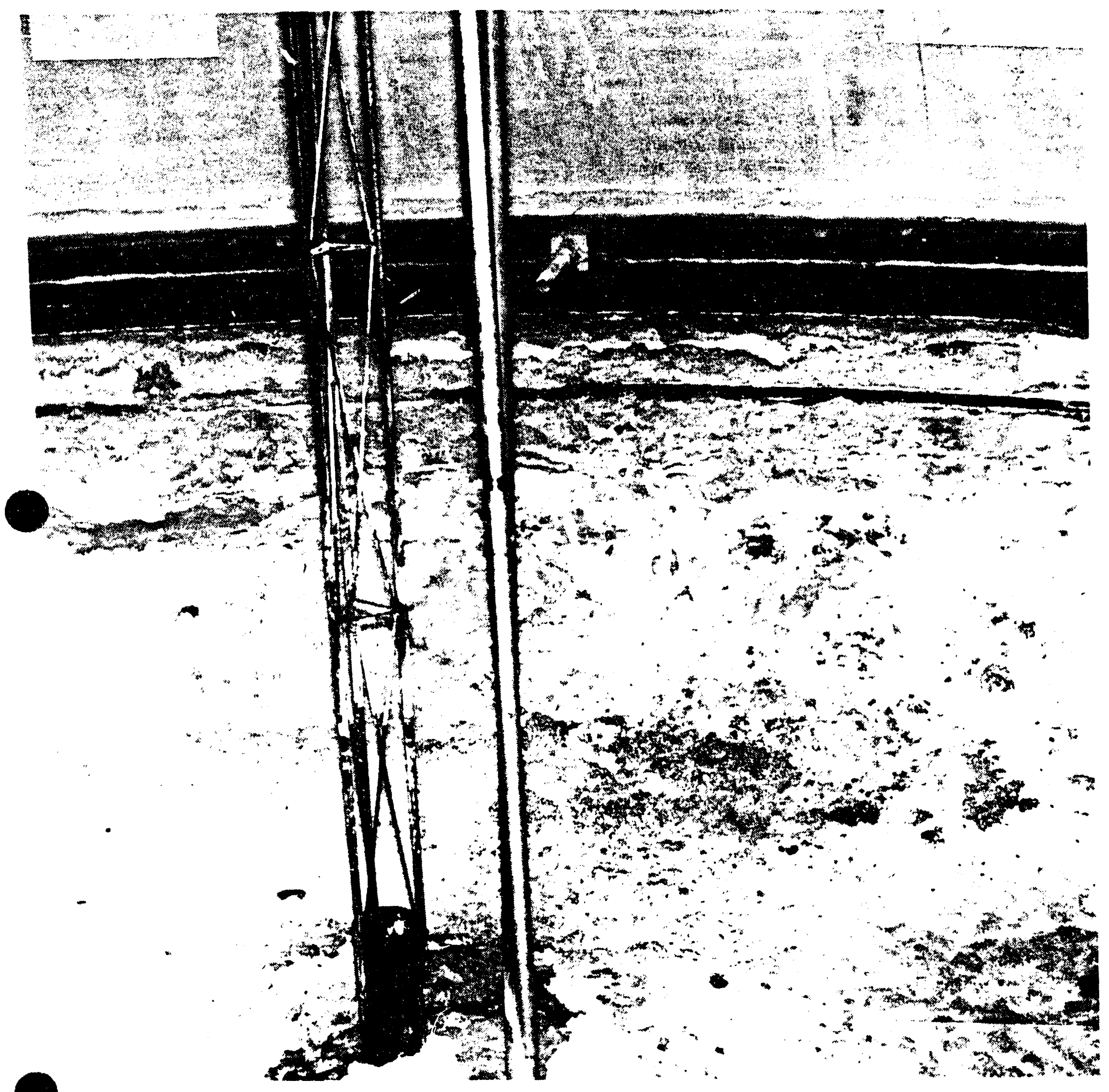


WHC-EP-0625

This page intentionally left blank. 


\section{Tank 241-BY-106}

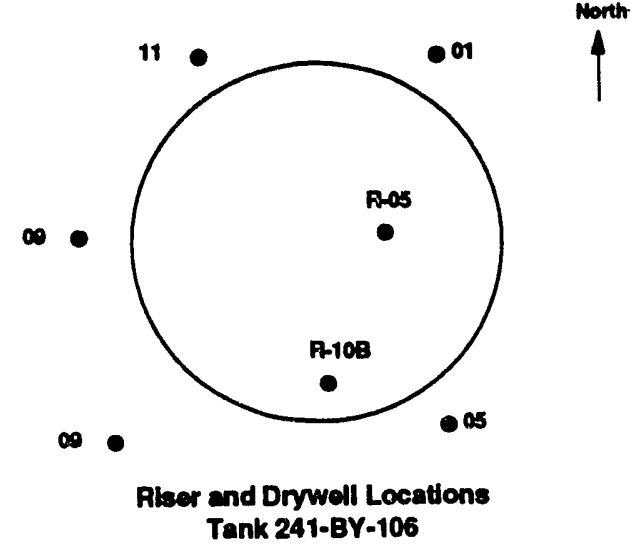

Number of External Drywells: 5

Number of Lateral Wells: None

\section{Tank Status}

\section{Watch List: Ferrocyanide}

Contents

Type: Non-Complexed Waste

Total Waste: $642 \mathrm{~K}$ gallons

Supernate Volume: OK gallons

Drainable Interstitial Liquid: $235 \mathrm{~K}$ gallons

Isolation Status

Date Partially Interim Isolated: 12/15/82

Surface Level/Leak Status

Integrity Category: Assumed Leaker

Date Declared Confirmed or Assumed Leaker: 1984

Leak Volume Estimate: $7.8 \mathrm{~K}$ gallons $=1 / 19$ th of $150 \mathrm{~K}$ gallons

Manual Tape Surface Level: 244.25 Inches (12/28/92)

Last Photographed: 11/04/82

Photo Interpretation Comments: See attached photo.

Temperature Status

Highest temperature during 1992: $131.3^{\circ} \mathrm{F}(03 / 15 / 92)$

Comments: Temperatures are stable.

Drywell Status

Comments: Current drywell proflles were stable and consistent with established baseline profiles.
Tank Description

Type: Single Shell

In-service: 1950

Diameter: $7^{\circ}$

Usable Depth: 23'

Capacity: $758 \mathrm{~K}$ gallons

Bottom shape: Dish

Hanford Coordinates:

46,102 ' North

53,349' West

Ventilation: Passive

\section{Leak Detection System}

Surface Level:

FIC Riser- None

Manual Tape Riser- R-05

LOW Riser(s)- R-10D 
WHC-EP-0625

This page intentionally left blank. 


\title{
Tank: 241-BY-106
}

\author{
November 1982
}

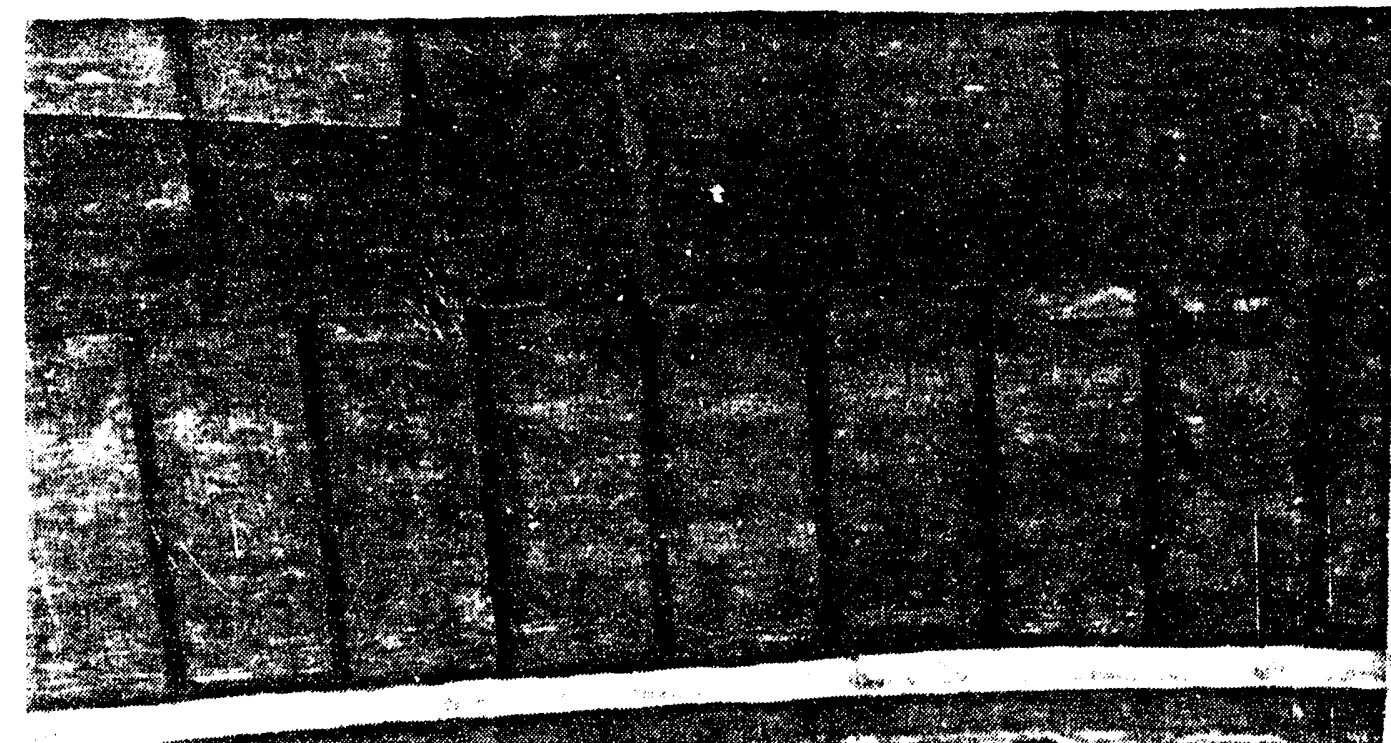

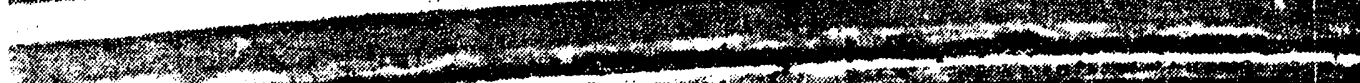

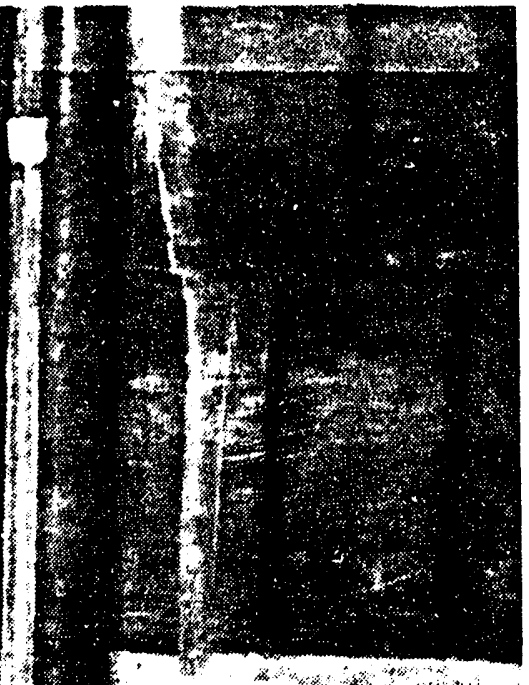

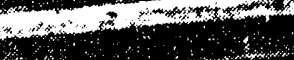
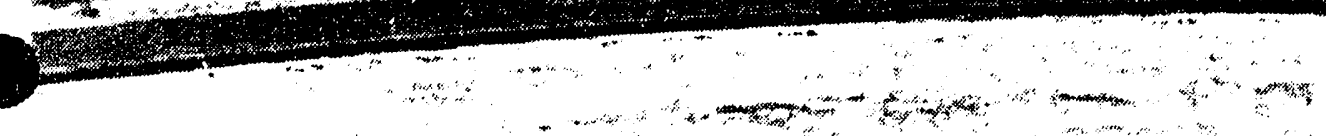

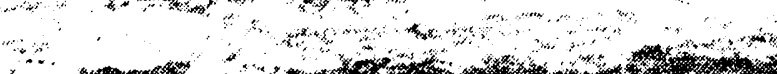

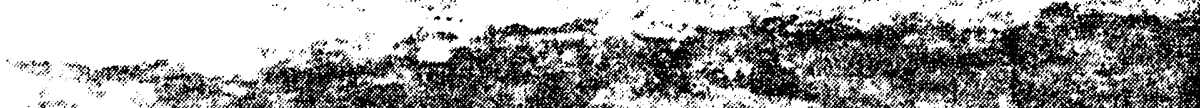
r 3.

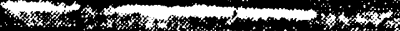
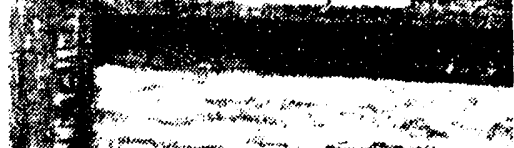

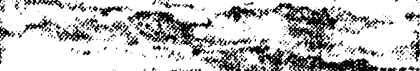
3

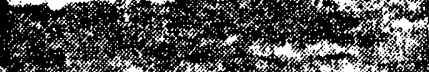

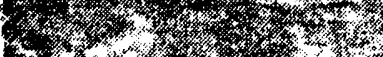

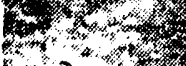

-

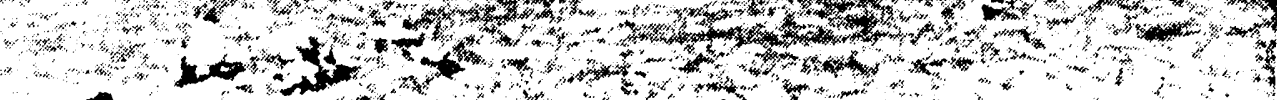

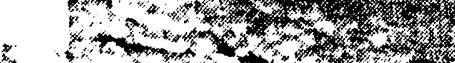

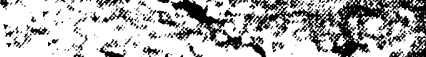

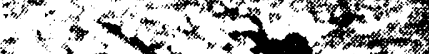

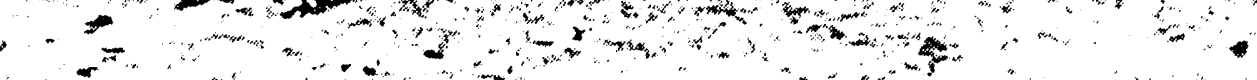

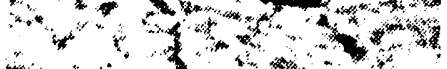

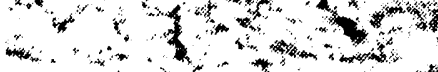
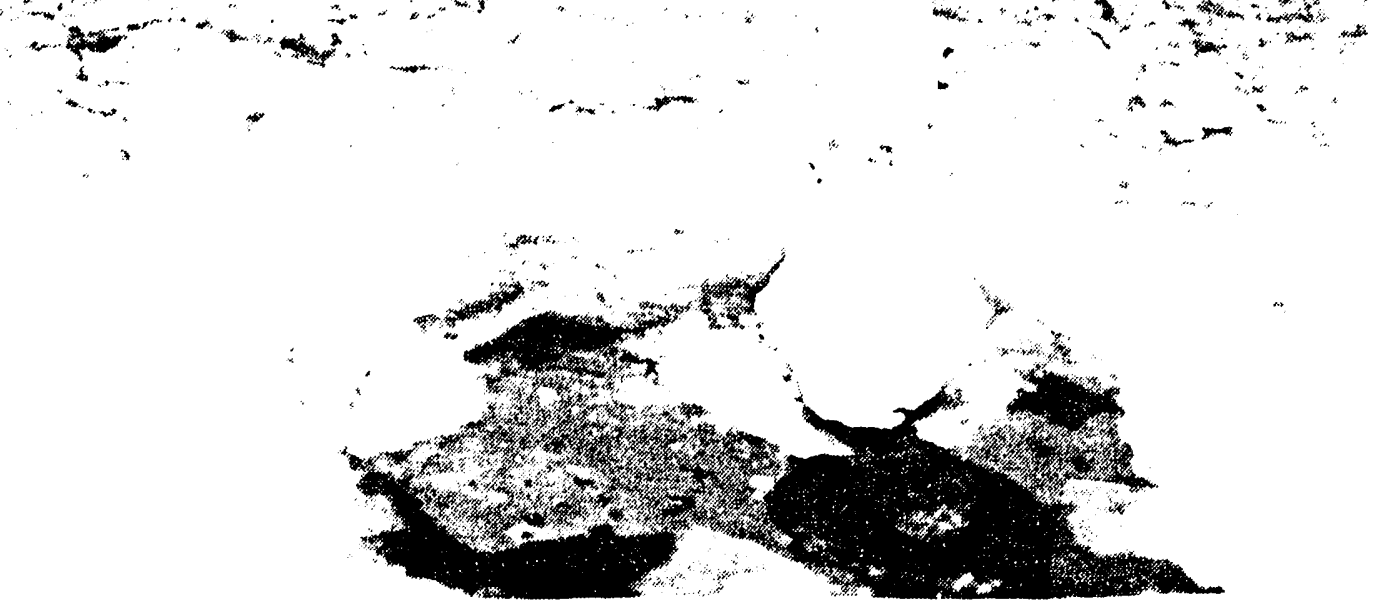

$104.475-7 \mathrm{CN}$ 
WHC-EP-0625

This page intentionally left blank. 


\section{Tank 241-BY-107}

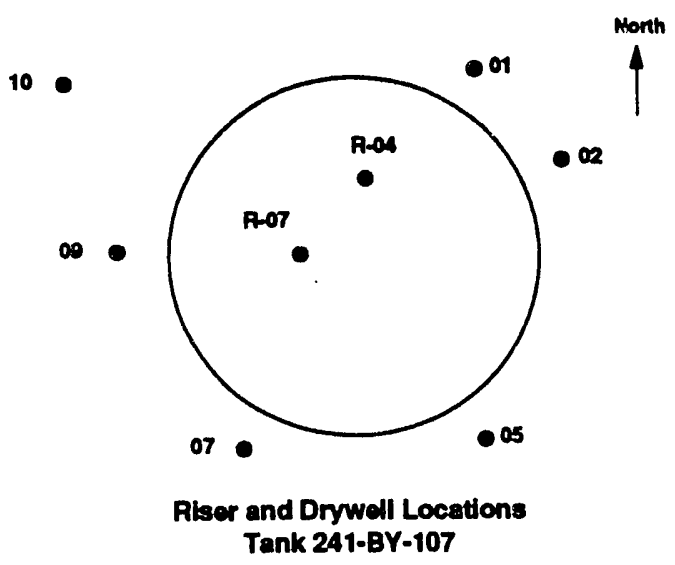

Number of External Drywells: 6 Number of Lateral Wells: None

\section{Tank Status}

Watch List: Ferrocyanide

\section{Contents}

Type: Non-Complexed Waste

Total Waste: 266K gallons

Supernate Volume: OK gallons

Drainable Interstitial Liquid: $25 \mathrm{~K}$ gallons

Isolation Status

Date Interim Stabilized: $07 / 79$

Date Interm Isolated: $12 / 15 / 82$

\section{Surface Level/Leak Status}

Integrity Category: Assumed Leaker

Date Declared Confirmed or Assumed Leaker: 1984

Leak Volume Estimate: 15,100 Gallons (1989)

Manual Tape Surface Level: 104.00 Inches (10/05/92)

Last Photographed: $10 / 15 / 86$

Photo Interpretation Comments: See attached photo.

Temperature Status

Highest temperature during 1992: $98.5^{\circ} \mathrm{F}(04 / 25 / 92)$

Comments: Temperatures are stable.

\section{Drywell Status}

Comments: Current drywell profiles were stable and consistent with established baseline profiles. 
WHC-EP-0625

This page intentionally left blank. 


\section{Tank: 241-BY-107}

(1) (iolut lopti

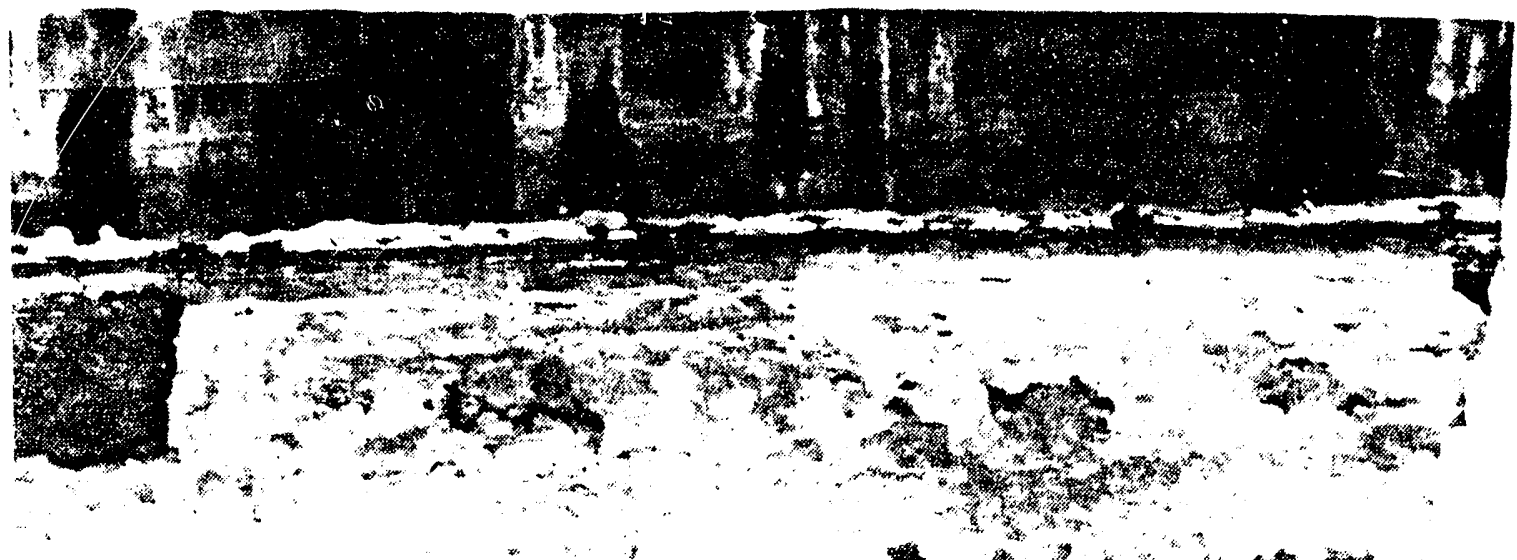

(3)

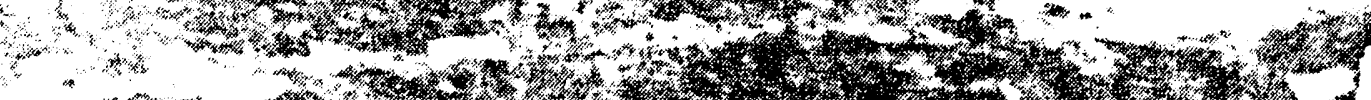

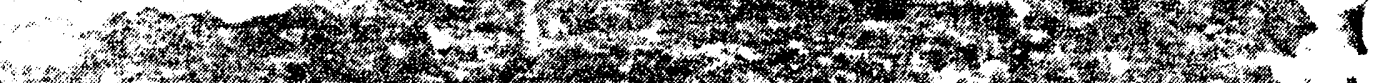

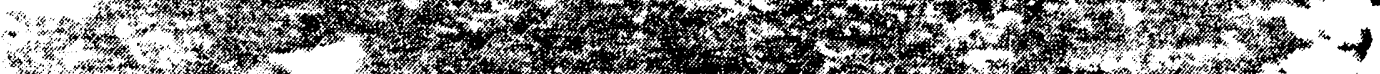

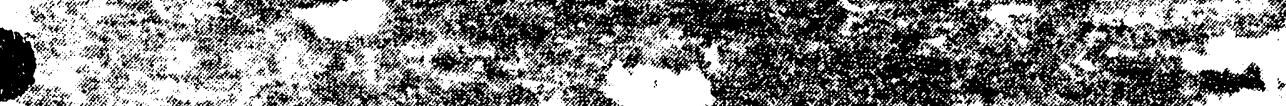

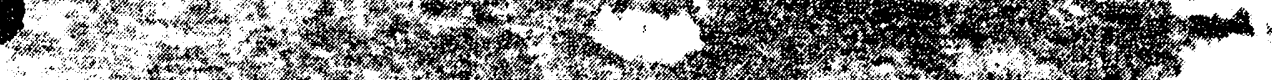

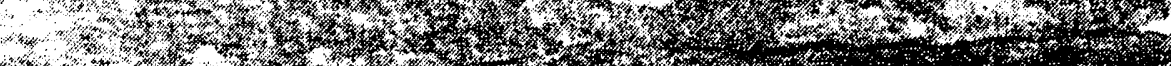

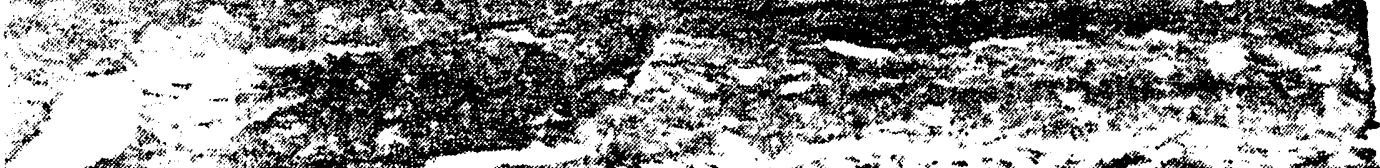
(1) (n)
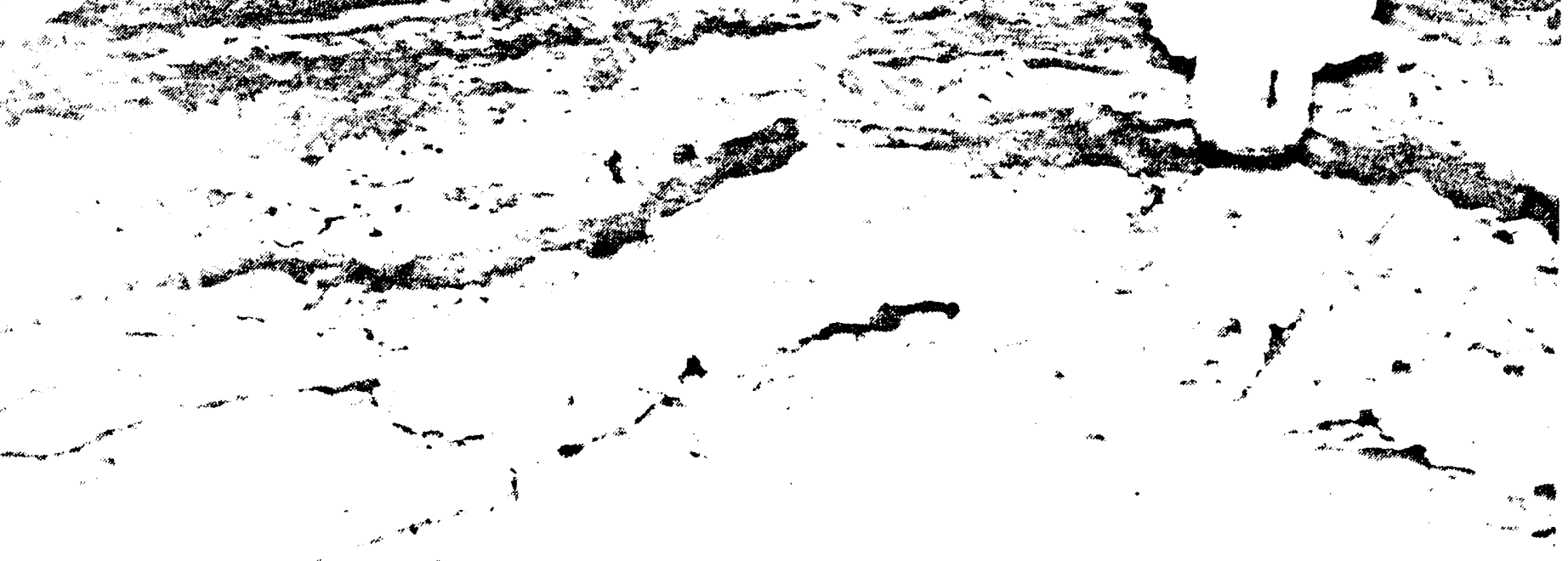
WHC-EP-0625

This page intentionally left blank. 


\section{Tank 241-BY-108}

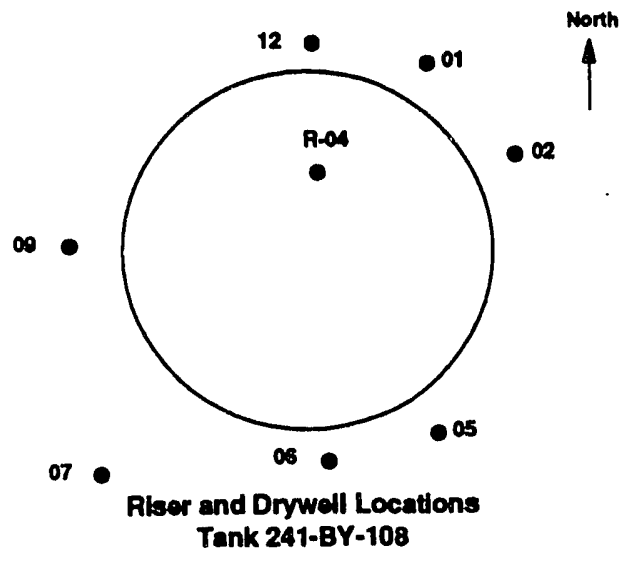

Number of External Drywells: 7

Number of Lateral Wells: None

\section{Tank Status}

\section{Watch List: Ferrocyanide}

Contents

Type: Non-Complexed Waste

Total Waste: $228 \mathrm{~K}$ gallons

Supernate Volume: OK gallons

Drainable Interstitial Liquid: 9K gallons

Isolation Status

Date Interim Stabilized: 02/06/85

Date Partially Interim Isolated: $12 / 15 / 82$

Surface Level/Leak Status

Integrity Category: Assumed Leaker

Date Declared Confirmed or Assumed Leaker: 1972

Leak Volume Estimate: < 5,000 Gallons (1983)

Manual Tape Surface Level: 87.50 Inches (12/01/92)

Last Photographed: 10/15/86

Photo Interpretation Comments: See attached photo.

Temperature Status

Highest temperature during 1992: $108^{\circ} \mathrm{F}(08 / 15 / 92)$

Comments: Temperatures are stable.

\section{Drywell Status}

Comments: Current drywell proflles were stable and consistent with established baseline profiles.
Tank Description

Type: Single Shell

Constructed: 1949

In-service: $04 / 51$

Diameter: $75^{\circ}$

Usable Depth: 23'

Capacity: $758 \mathrm{~K}$ gallons

Bottom shape: Dish

Hanford Coordinates:

$46,000^{\prime}$ North

53,451' West

Ventilation: Passive

\section{Leak Detection System}

Surface Level:

FIC Riser- None

Manual Tape Riser- R-04

LOW Riser(s)- None 
WHC-EP-0625

This page intentionally left blank. 


\section{Tank: 241-BY-108 \\ October 1986}






\section{WHC-EP-0625}

This page intentionally left blank. 


\section{Tank 241-BY-109}

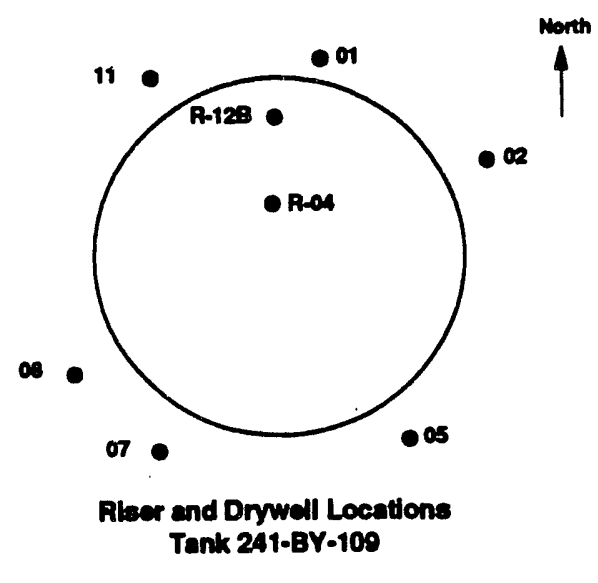

Number of External Drywells: 6

Number of Lateral Wells: None

\section{Tank Status}

Contents

Type: Non-Complexed Waste

Total Waste: $423 \mathrm{~K}$ gallons

Supernate Volume: OK gallons

Drainable Interstitial Liquid: $78 \mathrm{~K}$ gallons

\section{Surface Level/Leak Status}

Integrity Category: Sound

Last Photographed: 10/15/86

Photo Interpretation Comments:

Photographs from $04 / 27 / 83$ show more surface liquid than previous photographs with several patches of floating salt cake or scum-like material. The FIC plummet is contacting liquid. Photographs from 10/15/86 continue to show more surface liquid.

\section{Temperature Status}

Highest temperature during 1992: $102{ }^{\circ} \mathrm{F}(08 / 30 / 92)$

Comments: Temperatures are stable.

\section{Drywell Status}

Comments: Current drywell profiles were stable and consistent with established baseline profiles. 


\section{WHC-EP-0625}

This page intentionally left blank. 


\section{Tank 241-BY-110}

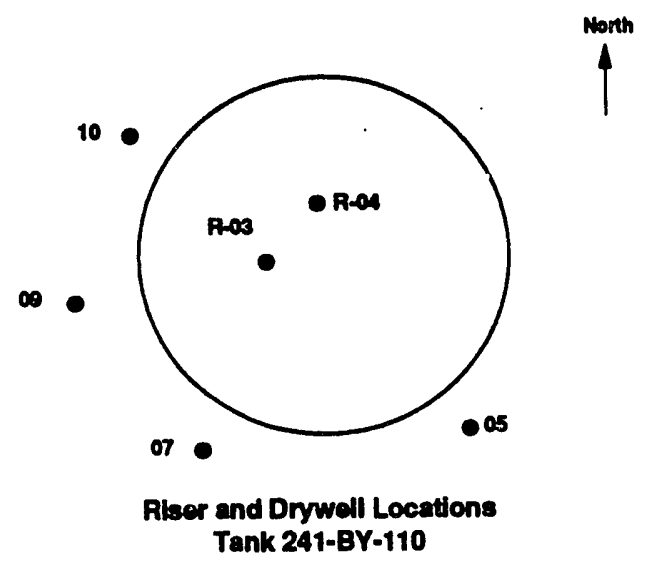

Number of External Drywells: 4

Number of Lateral Wells: None

\section{Tank Status}

Watch List: Ferrocyanide

Contents

Type: Non-Complexed Waste

Total Waste: $398 \mathrm{~K}$ gallons

Supernate Volume: OK gallons

Drainable Interstitial Liquid: $9 \mathrm{~K}$ gallons

Isolation Status

Date Interim Stabilized: 01/25/85

\section{Surface Level/Leak Status}

Integrity Category: Sound

Surface Level Comments: Out of Service on 12/30/92.

Last Photographed: 07/26/84

Photo Interpretation Comments: See attached photo.

Temperature Status

Highest temperature during 1992: $120^{\circ} \mathrm{F}(08 / 30 / 92)$

Comments: Temperatures are stable.

Drywell Status

Comments: Current drywell profles were stable and consistent with established baseline profiles. 


\section{WHC-EP-0625}

This page intentionally left blank. 


\section{Tank: 241-BY-110}

July 1984

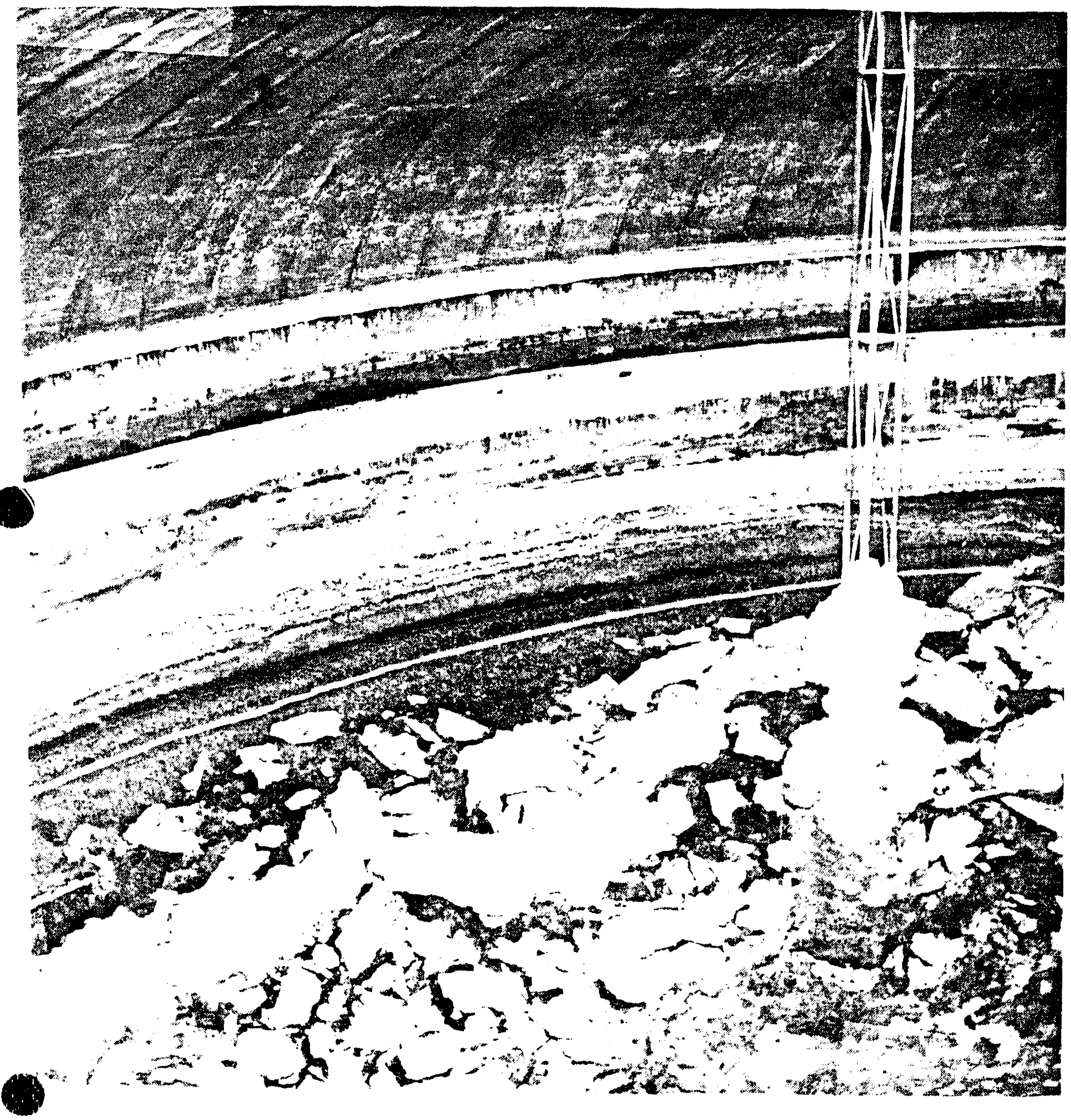


WHC-EP-0625

This page intentionally left blank. 


\section{Tank 241-BY-111}

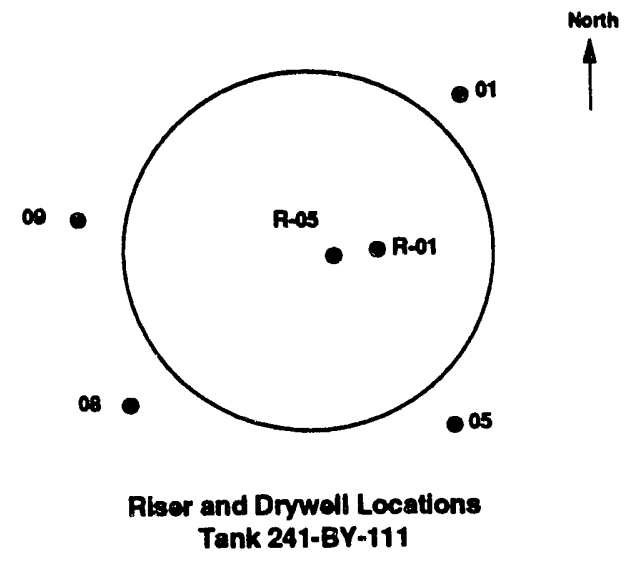

Tank 241-BY-111
Tank Description

Type: Single Shell

Constructed: 1949

In-service: 1951

Diameter: $7^{\circ}$

Usable Depth: '23'

Capacity: $758 \mathrm{~K}$ gallons

Bottom shape: Dish

Hanford Coordinates: $46,000^{\prime}$ North 53,553' West

Ventilation: Passive

\section{Leak Detection System}

Surface Level:

FIC Riser- None

Manual Tape Riser- R-05

LOW Riser(s)- R-01

Number of External Drywells: 4

Number of Lateral Wells: None

\section{Tank Status}

Watch List: Ferrocyanide

Contents

Type: Non-Complexed Waste

Total Waste: $459 \mathrm{~K}$ gallons

Supernate Volume: OK gallons

Drainable Interstitial Liquid: OK gallons

Isolation Status

Date Interim Stabilized: 01/25/85

\section{Surface Level/Leak Status}

Integrity Category: Sound

Last Photographed: $10 / 31 / 86$

Photo Interpretation Comments: See attached photo.

Temperature Status

Highest temperature during 1992: $89^{\circ} \mathrm{F}(08 / 01 / 92)$

Comments: Temperatures are stable.

\section{Drywell Status}

Comments: Current drywell profiles were stable and consistent with established baseline profiles. 


\section{WHC-EP-0625}

This page intentionally left blank. 


\section{Tank: 241-BY-111}

October 1986
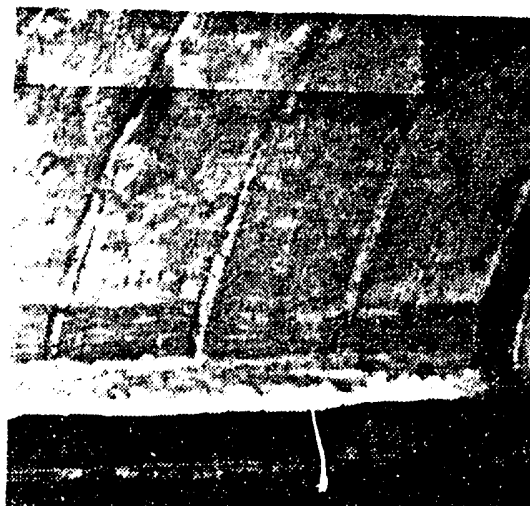

4.

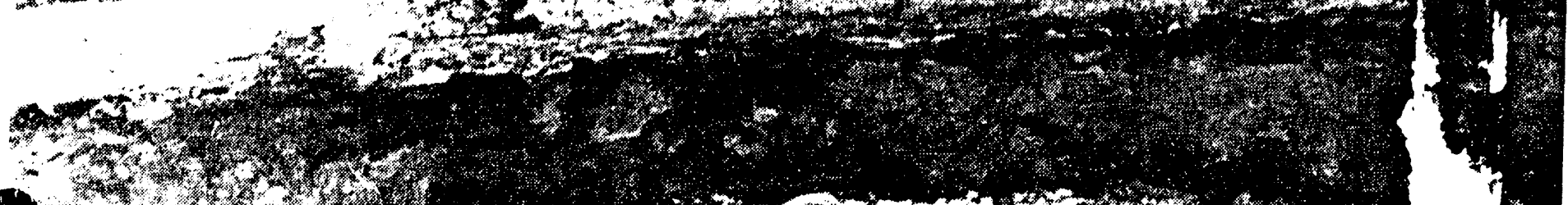
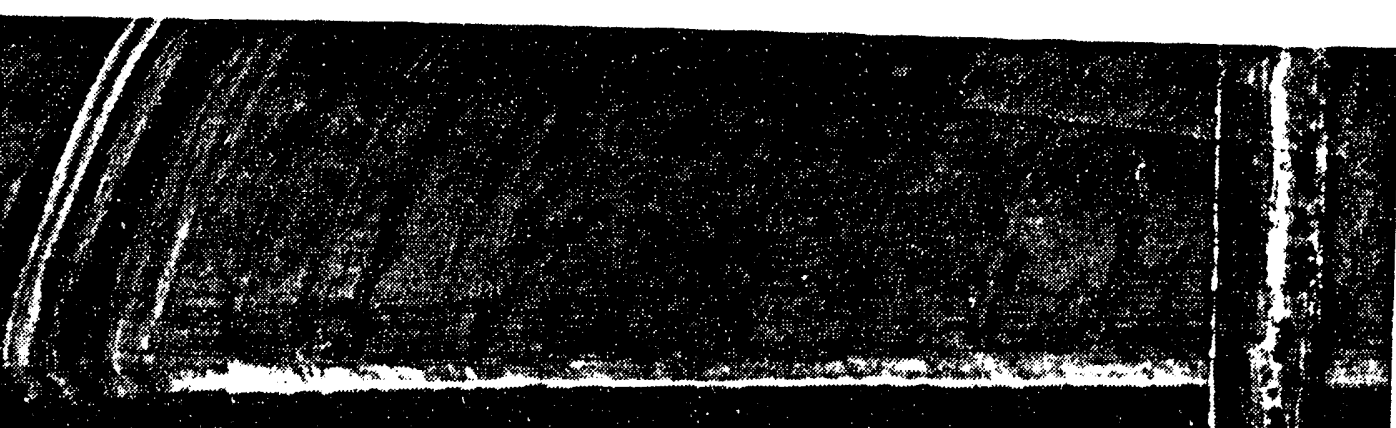

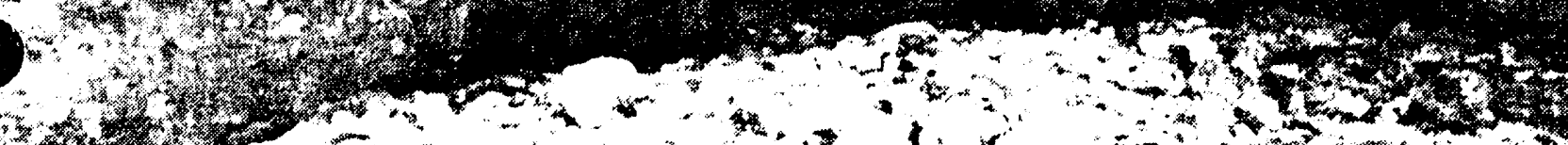

(3)

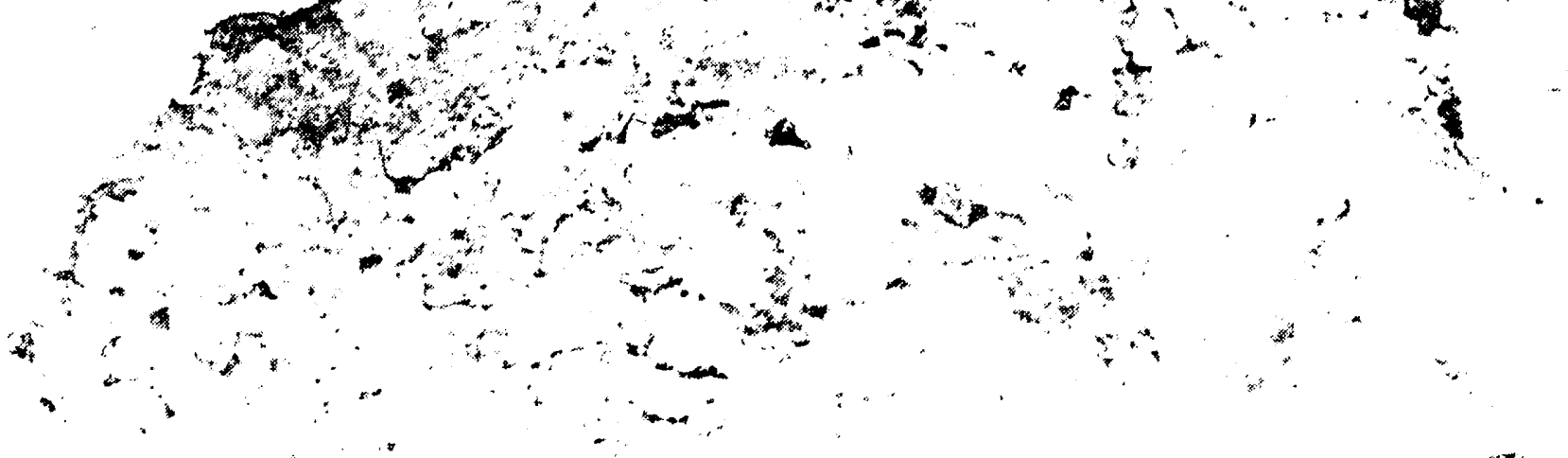


WHC-EP-0625

This page intentionally left blank. 


\section{Tank 241-BY-112}

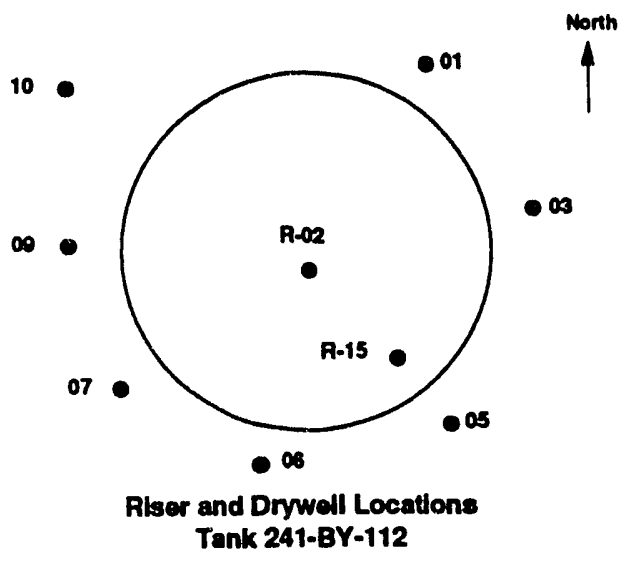

Number of External Drywells: 7

Number of Lateral Wells: None

Tank Status

Watch List: Ferrocyanide

Contents

Type: Non-Complexed Waste

Total Waste: $291 \mathrm{~K}$ gallons

Supernate Volume: OK gallons

Drainable Interstitial Liquid: $8 \mathrm{~K}$ gallons

Isolation Status

Date Interim Stabilized: 06/13/84

\section{Surface Level/Leak Status}

Integrity Category: Sound

Manual Tape Surface Level: 113.00 Inches (12/01/92)

Last Photographed: 04/14/88

Photo Interpretation Comments: See attached photo.

Temperature Status

Highest temperature during 1992: $85^{\circ} \mathrm{F}(08 / 30 / 92)$

Comments: Temperatures are stable.

Drywell Status

Comments: Current drywell profiles were stable and consistent with established basellne profiles.
Tank Description

Type: Single Shell

Constructed: 1949

In-service: 1951

Diameter: $75^{\circ}$

Usable Depth: 23'

Capacity: $758 \mathrm{~K}$ gallons

Bottom shape: Dish

Hanford Coordinates:

$46,102^{\prime}$ North

53,553' West

Ventilation: Passive

\section{Leak Detection System}

Surface Level:

FIC Riser- None

LOW Riser(s)- R-15
Manual Tape Riser- R-02 
WHC-EP-0625

This page intentionally left blank. 
Whic H.M

Tank: 241-BY-112

April 1988
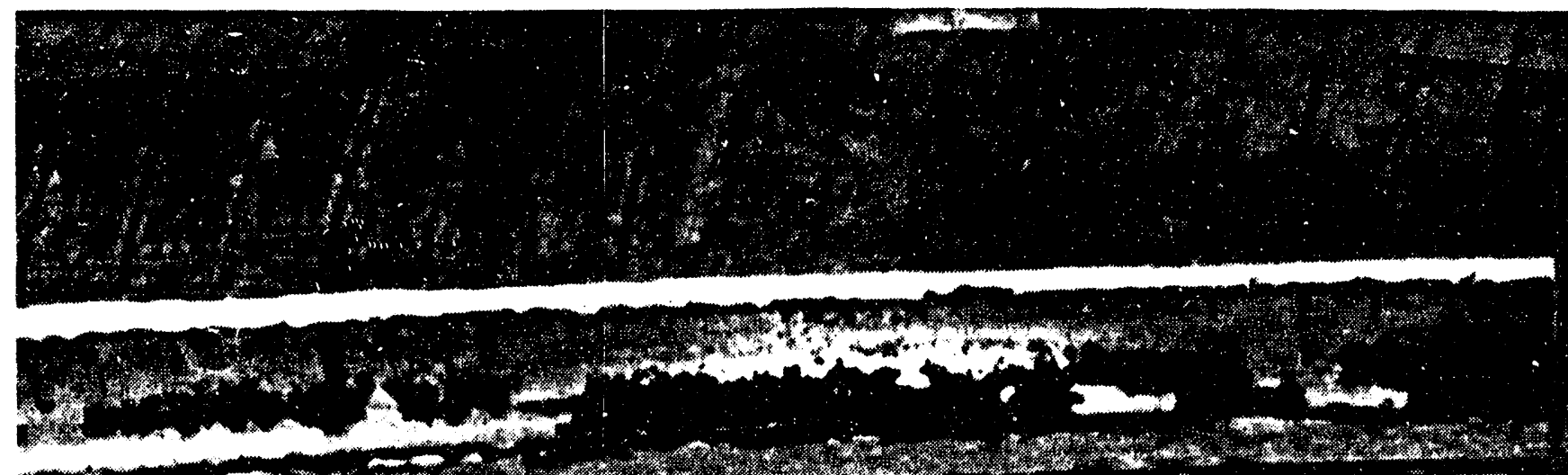

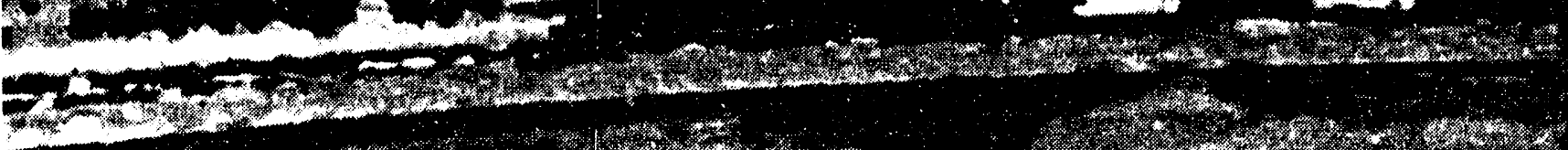

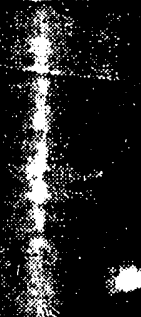

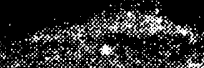

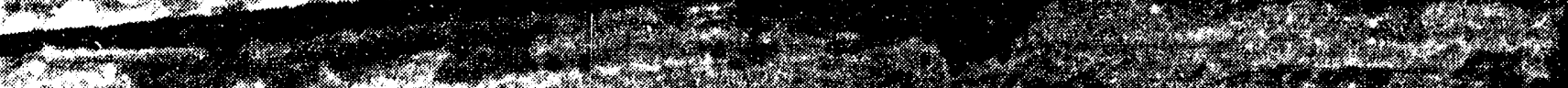

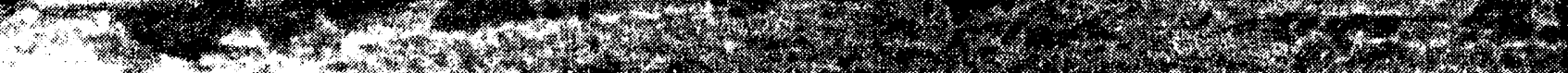

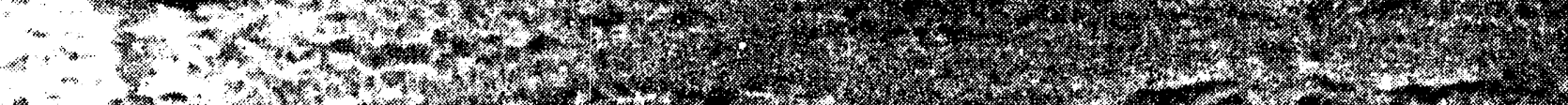

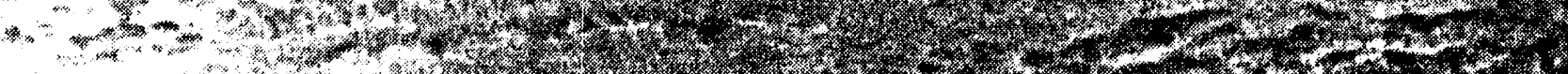

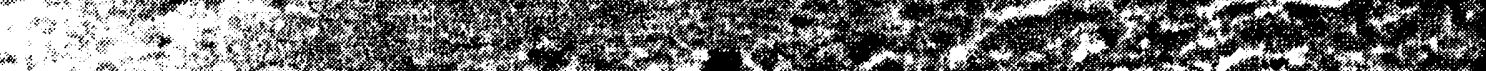

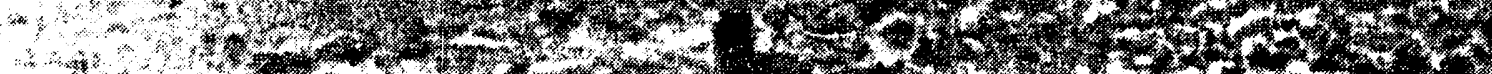

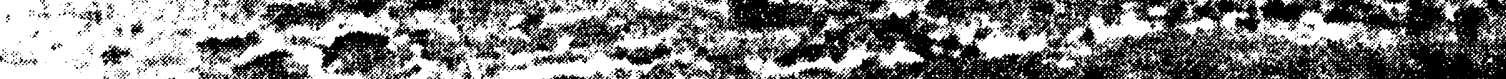

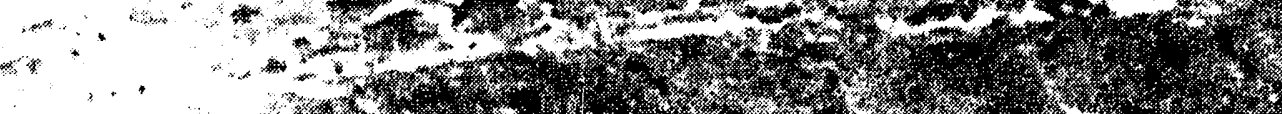

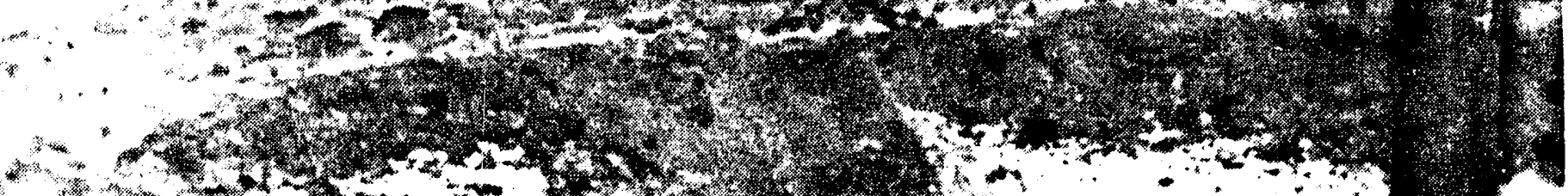

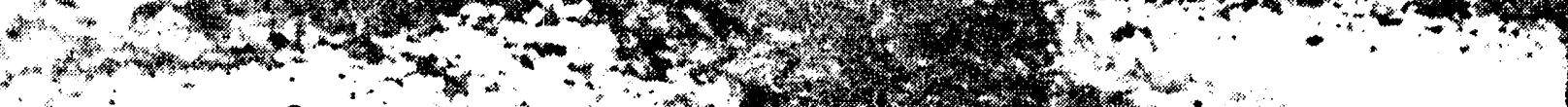

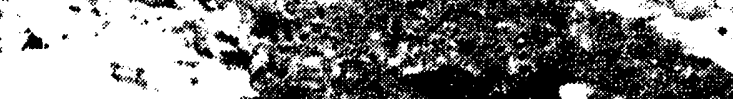

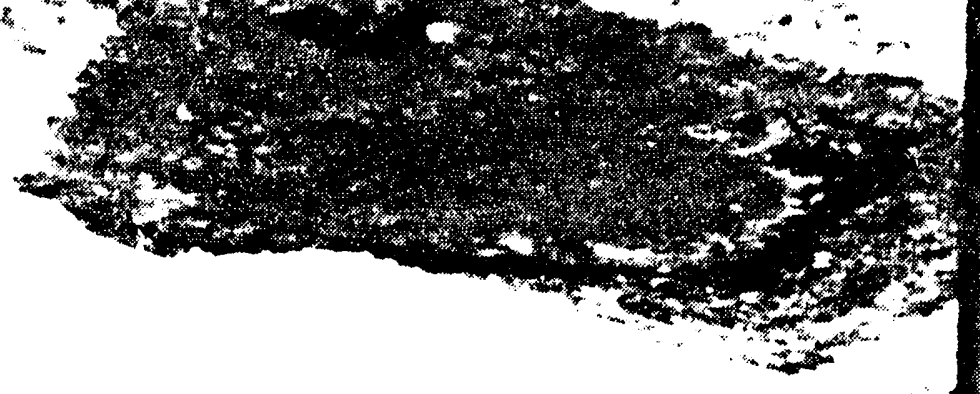

$+4$

$8801299-13 \mathrm{CN}$ 
WHC-EP-0625

This page intentionally left blank. 


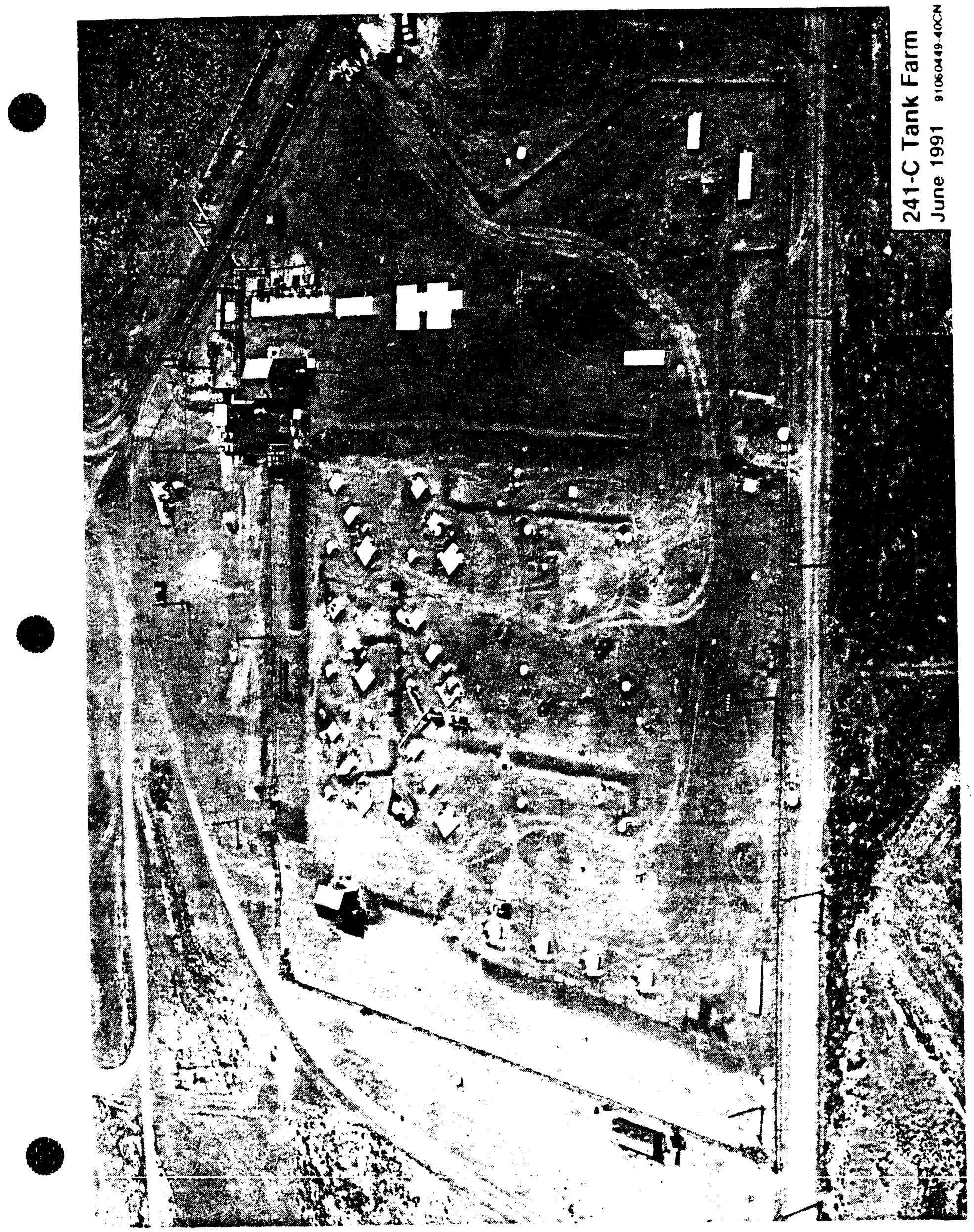


WHC-EP-0625

This page intentionally left blank. 


\section{Tank 241-C-101}

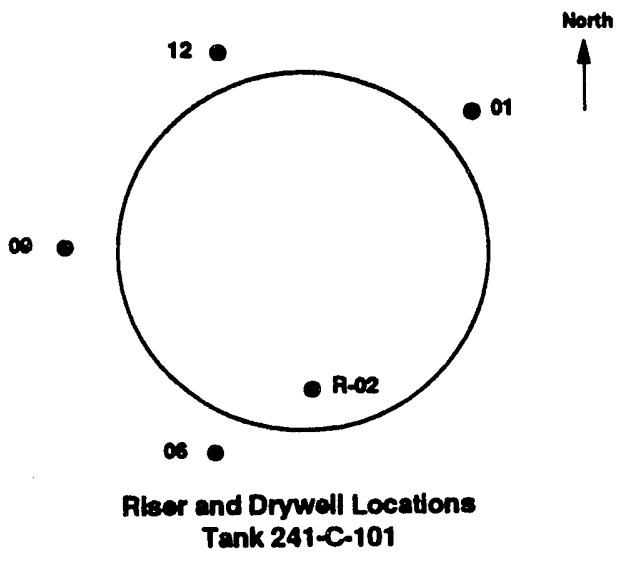

Number of External Drywells: 4
Tank Description

Type: Single Shell

Constructed: 1944

In-service: $03 / 46$

Out of Service: $12 / 69$

Diameter: $75^{\circ}$

Usable Depth: $16^{\circ}$

Capacity: $530 \mathrm{~K}$ gallons

Bottom shape: Dish

Hanford Coordinates: 42,719 North $48,327^{\prime}$ 'West

Ventilation: Passive

\section{Leak Detection System}

Surface Level:

FIC Riser- None

Manual Tape Riser- R-02

LOW Riser(s)- None

Number of Lateral Wells: None

\section{Tank Status}

Contents

Type: Non-Complexed Waste

Total Waste: $88 \mathrm{~K}$ gallons

Supernate Volume: OK gallons

Drainable Interstitial Liquid: $3 \mathrm{~K}$ gallons

Isolation Status

Date Interim Stabilized: $11 / 29 / 83$

Date Interm Isolated: $12 / 15 / 82$

\section{Surface Level/Leak Status}

Integrity Category: Assumed Leaker

Date Declared Confirmed or Assumed Leaker: 1980

Leak Volume Estimate: 20.000 Gallons (1986)

Manual Tape Surface Level: 26.00 Inches (11/23/92)

Last Photographed: $11 / 17 / 87$

Photo Interpretation Comments:

Photographs reveal a sludge surface with a very small area of shallow liquid visible.

Temperature Status

Highest temperature during 1992: $89^{\circ} \mathrm{F}(11 / 05 / 92)$

Comments: Temperatures are stable.

Drywell Status

Comments: Current drywell profiles were stable and consistent with established baseline profiles. 


\section{WHC-EP-0625}

This page intentionally left blank. 


\section{Tank 241-C-102}

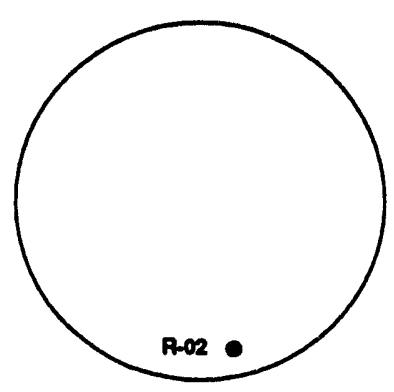

Riser and Drywell Locattons Tank 241-C-102

Number of External Drywells: None Number of Lateral Wells: None

\section{Tank Status}

Contents

Type: Dilute Complexed Waste

Total Waste: 423K gallons

Supernate Volume: OK gallons

Drainable Interstitial Liquid: $37 \mathrm{~K}$ gallons

Isolation Status

Date Partially Interim Isolated: 12/15/82

\section{Surface Level/Leak Status}

Integrity Category: Sound

FIC Surface Level: 152.00 Inches (12/28/92)

Surface Level Comments: Intrusion Mode

Last Photographed: 05/18/76

Photo Interpretation Comments:

Photographs reveal a dry, lumpy surface of sludge with very shallow puddles of liquid visible. The FIC plummet is contacting sludge and a discarded tape and teflon $^{\mathrm{TM}}$ gasket is in the immediate vicinity of the plummet.

\section{Temperature Status}

Highest temperature during 1992: $96^{\circ} \mathrm{F}(11 / 05 / 92)$

Comments: Temperatures are stable.

\section{Drywell Status}

Comments: Current drywell profiles were stable and consistent with established baseline profiles.
Tank Description

Type: Single Shell

Constructed: 1944

In-service: $05 / 46$

Out of Service: 1976

Diameter: $7^{\circ}$

Usable Depth: $16^{\prime}$

Capacity: $530 \mathrm{~K}$ gallons

Bottom shape: Dish

Hanford Coordinates:

42.790' North

48,256 West

Ventilation: Passive

\section{Leak Detection System}

Surface Level:

FIC Riser- R-02

Manual Tape Riser- None

LOW Riser(s)- None 
WHC-EP-0625

This page intentionally left blank. 


\section{Tank 241-C-103}

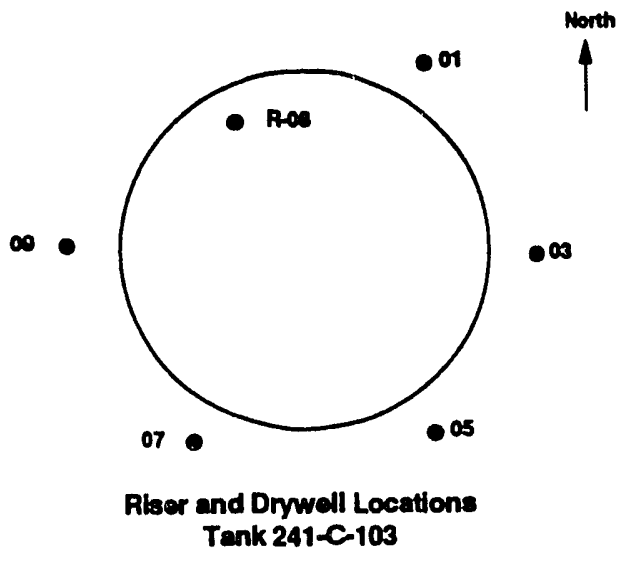

Number of External Drywells: 5 Number of Lateral Wells: None

\section{Tank Status}

Watch List: Organic Salts

Contents

Type: Non-Complexed Waste

Total Waste: $195 \mathrm{~K}$ gallons

Supernate Volume: 133K gallons

Drainable Interstitial Liquid: OK gallons

Isolation Status

Date Partially Interim Isolated: $12 / 15 / 82$

Surface Level/Leak Status

Integrity Category: Sound

FIC Surface Level: 66.20 Inches $(12 / 28 / 92)$

Last Photographed: 07/28/87

Temperature Status

Highest temperature during 1992: $128^{\circ} \mathrm{F}(10 / 10 / 92)$

Comments: Temperatures are stable.

\section{Drywell Status}

Comments: Current drywell profiles were stable and consistent with established baseline profiles. 
WHC-EP-0625

This page intentionally left blank. 
WHC-EP-0625

\section{Tank: 241-C-103 \\ July 1987}

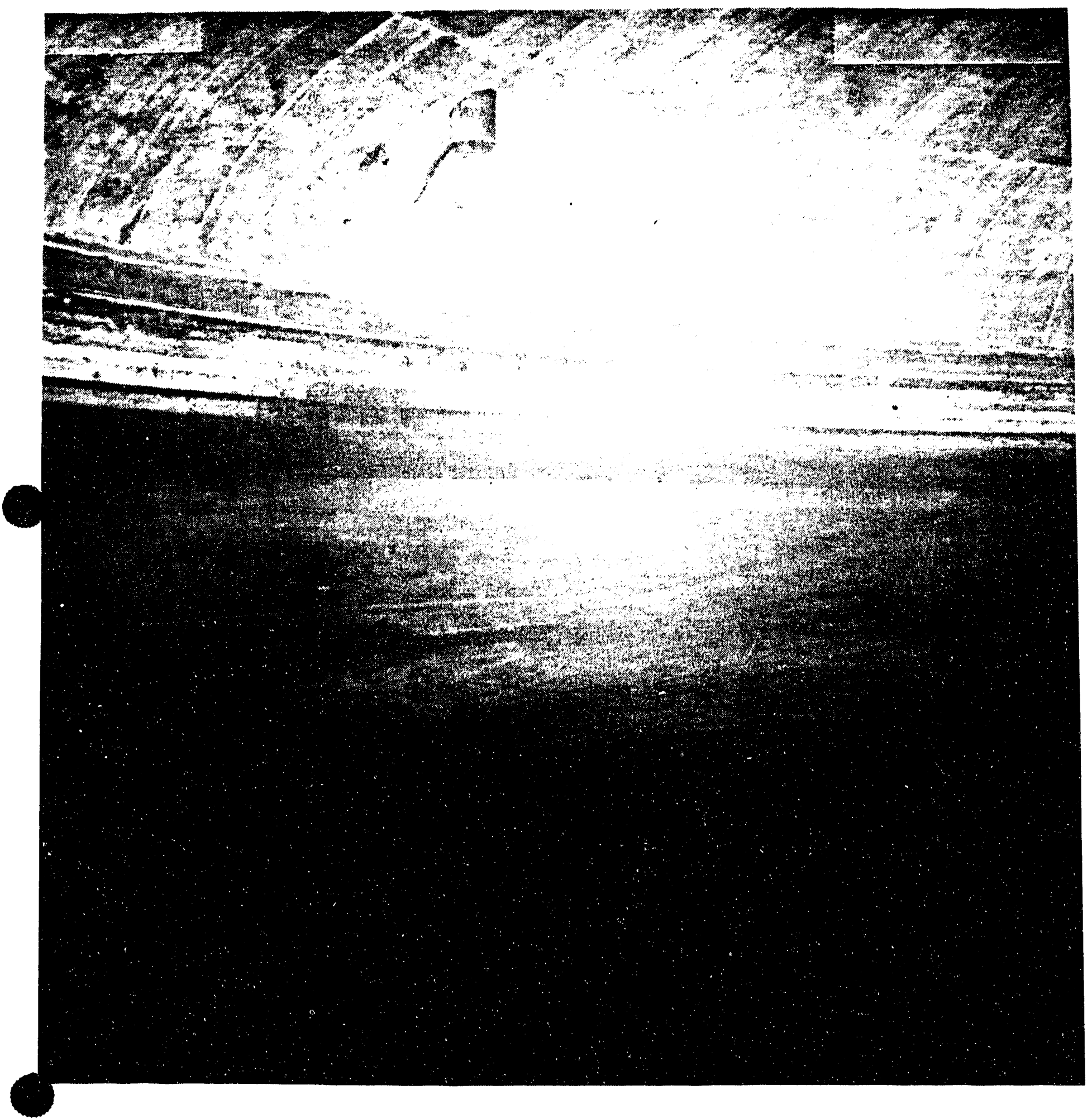


WHC-EP-0625

This page intentionally left blank. 


\section{Tank 241-C-104}

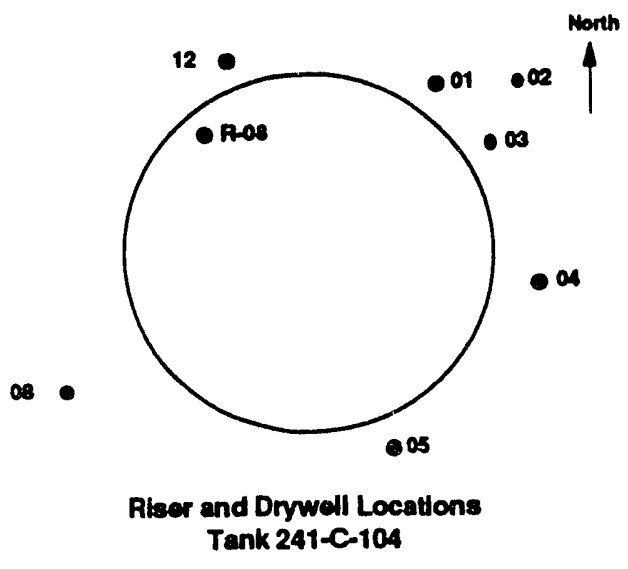

Number of External Drywells: 7

Number of Lateral Wells: None

\section{Tank Status}

Contents

Type: Complexant Concentrate Waste

Total Waste: 295K gallons

Supernate Volume: OK gallons

Drainable Interstitial Liquid: $11 \mathrm{~K}$ gallons

Isolation Status

Date Partially Interim Isolated: 12/15/92

Date Interim Stabilized: 09/89

Surface Level/Leak Status

Integrity Category: Sound

FIC Surface Level: 87.50 Inches (12/28/92)

Last Photographed: 07/25/90

Photo Interpretation Comments: See attached photo.

Temperature Status

Highest temperature during 1992: $92^{\circ} \mathrm{F}(11 / 06 / 92)$

Comments: Temperatures are stable.

\section{Drywell Status}

Comments: Current drywell profiles were stable and consistent with established baseline profiles.
Tank Description

Type: Single Shell

Constructed: 1944

In-service: $10 / 46$

Dlameter: $75^{\prime}$

Usable Depth: 16"

Capacity: $530 \mathrm{~K}$ gallons

Hanford Coordinates:

$42,790^{\prime}$ North

48,398' West

Ventilation: Passive

\section{Leak Detection System}

Surface Level:

FIC Riser- R-08

Manual Tape Riser- None

LOW Riser(s)- None
Bottom shape: Dish 
WHC-EP-0625

This page intentionally left blank. 


\section{Tank 241-C-105}

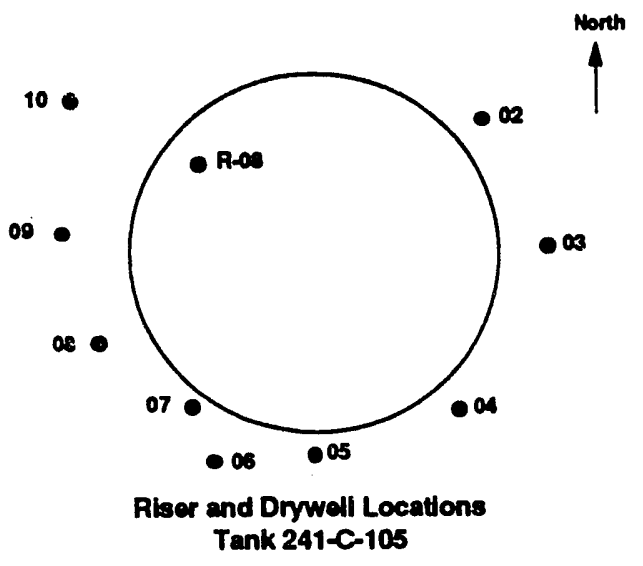

Tank Description

Type: Single Shell

Constructed: 1944

In-service: 02/46

Diameter: $7^{\circ}$

Usable Depth: 16'

Capactty: $530 \mathrm{~K}$ gallons

Bottom shape: Dish

Hanford Coordinates:

$42,861^{\prime}$ North

$48,327^{\circ}$ West

Ventilation: Passive

\section{Leak Detection System}

Surface Level:

FIC Riser- R-08

Manual Tape Riser- None

Low Riser(s)- None

Number of External Drywells: 9

Number of Lateral Wells: None

\section{Tank Status}

Contents.

Type: Non-Complexed Waste

Total Waste: $150 \mathrm{~K}$ gallons

Supernate Volume: OK gallons

Drainable Interstitial Liquid: $11 \mathrm{~K}$ gallons

Surface Level/Leak Status

Integrity Category: Sound

Manual Tape Surface Level: Not Avallable (Not Available)

FIC Surface Level: 47.90 Inches (12/29/92)

Last Photographed: 04/01/88

Photo Interpretation Comments:

Photographs from 06/27/79 show mostly liquid with exposed solids around the tank perimeter and several patches of floating scum or organic. Photographs from

\section{Temperature Status} $03 / 28 / 85$ show little change except for slightly more exposed sludge.

Highest temperature during 1992: $105.90^{\circ} \mathrm{F}(08 / 16 / 92)$

Comments: Temperatures are stable.

\section{Drywell Status}

Comments: Current drywell profiles were stable and consistent with established

baseline profiles. 
WHC-EP-0625

This page intentionally left blank. 


\section{Tank 241-C-106}

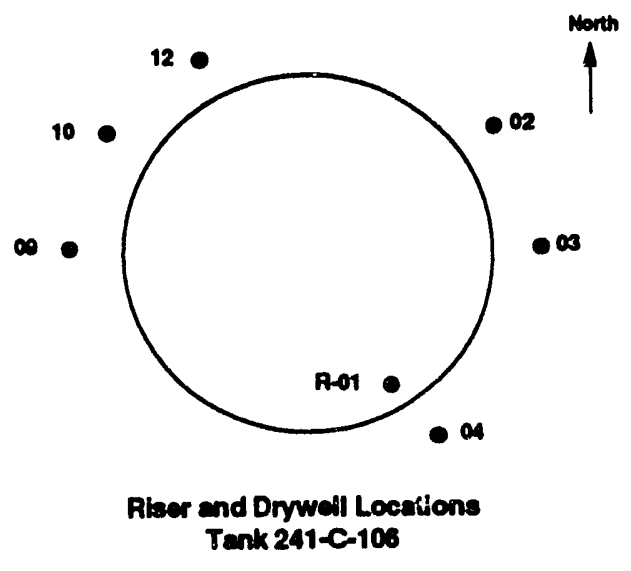

Number of External Drywells: 6

Number of Lateral Wells: None

\section{Tank Status}

\section{Watch List: High Heat}

Contents

Type: Non-Complexed Waste

Total Waste: $229 \mathrm{~K}$ gallons

Supernate Volume: $32 \mathrm{~K}$ gallons

Drainable Interstitial Liquid: $16 \mathrm{~K}$ gallons

Surface Level/Leak Status

Integrity Category: Sound

Manual Tape Surface Level: Not Avallable (Not Avallable)

FIC Surface Level: 77.70 Inches (12/29/92)

Last Photographed: 04/05/79

Photo Interpretation Comments: See attached photo.

Temperature Status

Highest temperature during 1992: $198^{\circ} \mathrm{F}(06 / 07 / 92)$

Comments: Temperatures are stable.

Drywell Status

Comments: Current drywell profles were stable and consistent with established baseline profiles.
Tank Description

Type: Single Shell

Constructed: 1944

In-service: $06 / 47$

Out of Service: 1979

Diameter: $75^{\circ}$

Usable Depth: 16'

Capacity: $530 \mathrm{~K}$ gallons

Bottom shape: Dish

Hanford Coordinates: 42,932' North $48,256^{\circ}$ West

Ventilation: Passive

\section{Leak Detection System}

Surface Level:

FIC Riser- R-01

Manual Tape Riser- None

LOW Riser(s)- None 
WHC-EP-0625

This page intentionally left blank. 
WHC-EP-0625

\section{Tank: 241-C-106 \\ April 1979}

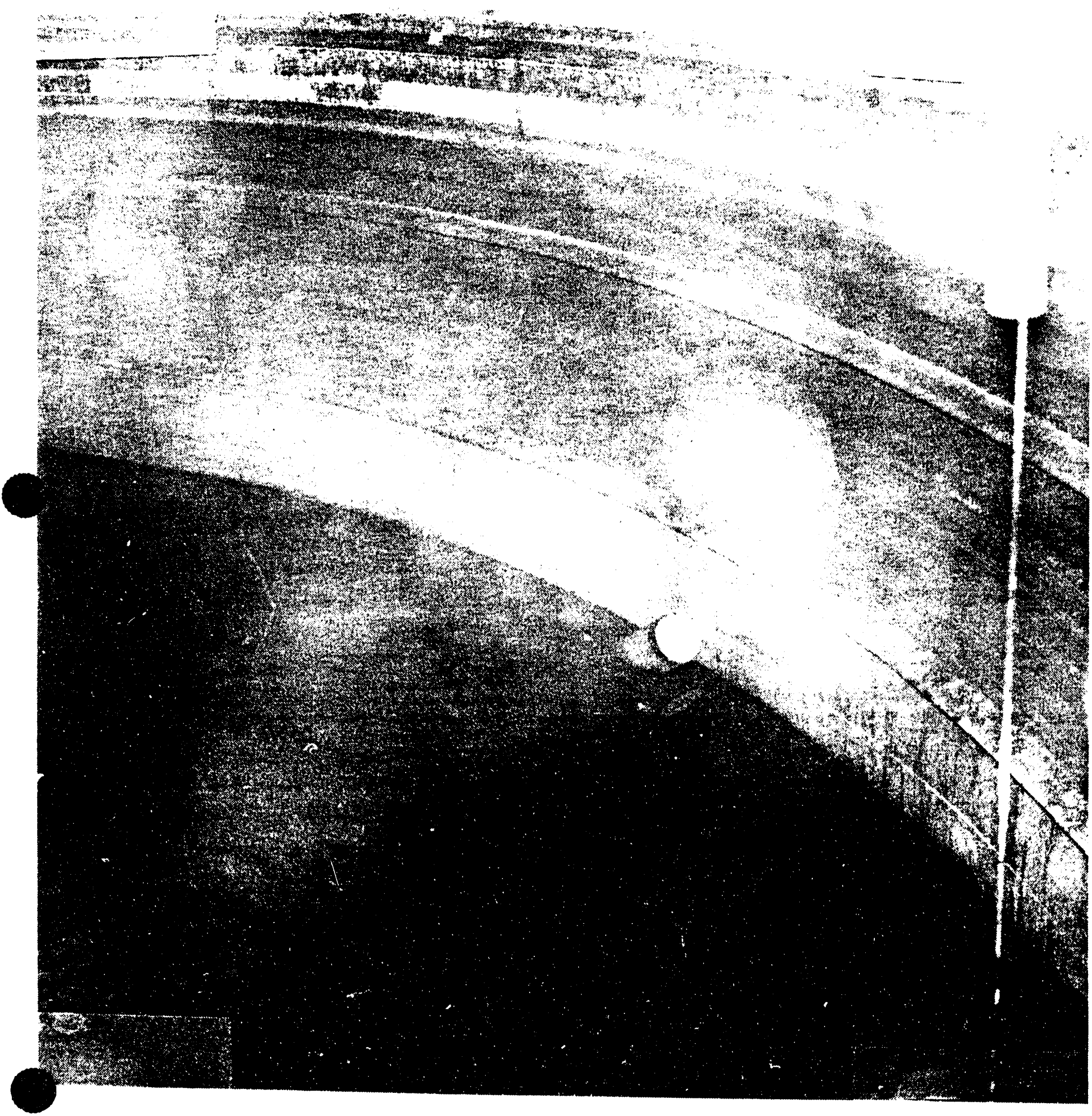




\section{WHC-EP-0625}

This page intentionally left blank. 


\section{Tank 241-C-107}

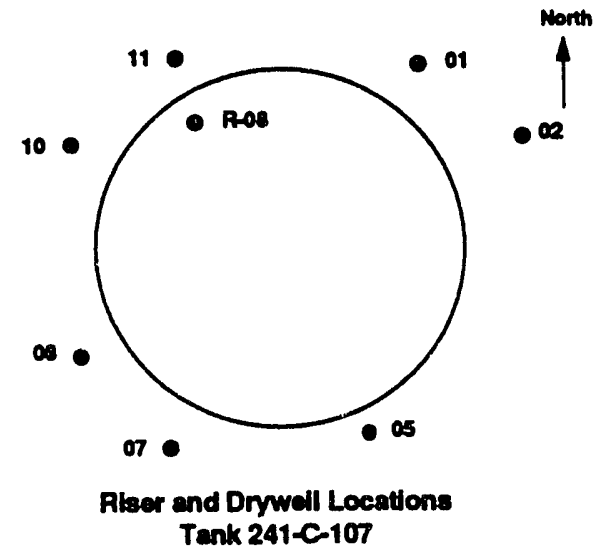

Number of External Drywells: 7

Number of Lateral Wells: None
Tank Description

Type: Single Shell

Constricted: 1944

In-service: $04 / 46$

Out of Service: 1976

Diameter: $75^{\prime}$

Usable Depth: 16'

Capacity: 530K gallons

Bottom shape: Dish

Hanford Coordinates: 42,861' North 48,469' West

Ventilation: Passive

\section{Leak Detection System}

Surface Level:

FIC Riser- R-08

Manual Tape Riser- None LOW Riser(s)- None

\section{Tank Status}

Contents

Type: Dilute Complexed Waste

Total Waste: $275 \mathrm{~K}$ gallons

Supernate Volume: OK gallons

Drainable Interstitial Liquid: $26 \mathrm{~K}$ gallons

Isolation Status

Date Partially Interim Isolated: 12/15/82

Surface Level/Leak Status

Integrity Category: Sound

FIC Surface Level: 95.30 Inches (12/21/92)

Surface Level Comments: Out of Service on 12/28/92

Temperature Status

Highest recent temperature: $129.8^{\circ} \mathrm{F}(09 / 25 / 93)$

Comments: Temperatures are stable.

Drywell Status

Comments: Current drywell profiles were stable and consistent with established baseline profiles. 
WHC-EP-0625

This page intentionally left blank. 


\section{Tank 241-C-108}

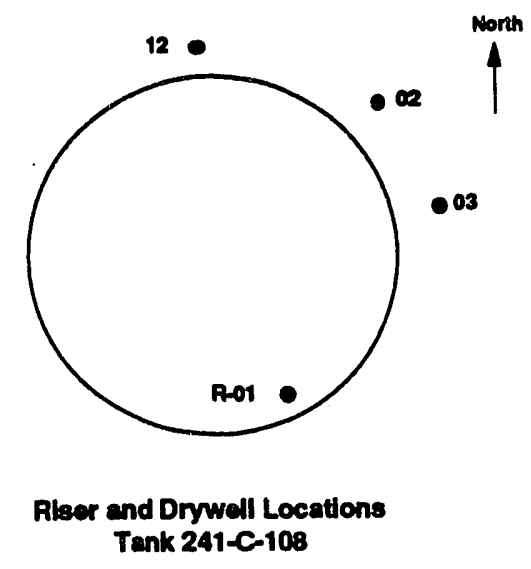

Number of External Drywells: 3 Number of Lateral Wells: None

\section{Tank Status}

Watch List: Ferrocyanide

Contents

Type: Non-Complexed Waste

Total Waste: $66 \mathrm{~K}$ gallons

Supernate Volume: OK gallons

Drainable Interstitial Liquid: OK gallons

Isolation Status

Date Interim Stabilized: 03/09/84

Date Interim Isolated: $12 / 15 / 82$

\section{Surface Level/Leak Status}

Integrity Category: Sound

Manual Tape Surface Level: 20.00 Inches (11/23/92)

Last Photographed: 12/05/74

Photo Interpretation Comments: See attached photo.

Temperature Status

Highest temperature during 1992: $79.7^{\circ} \mathrm{F}(10 / 23 / 92)$

Comments: Temperatures are stable.

\section{Drywell Status}

Comments: Current drywell profiles were stable and consistent with established baseline profiles. 
WHC-EP-0625

This page intentionally left blank. 


\section{VHHC-EP-0625}

\section{Tank: 241-C-108}

\section{December 1974}

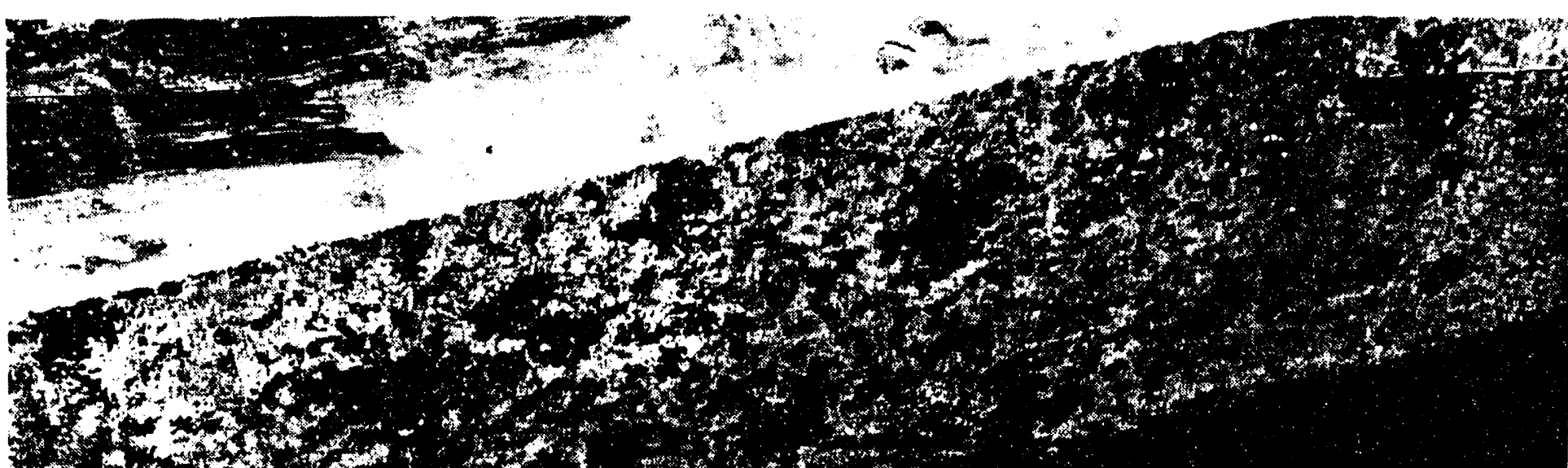

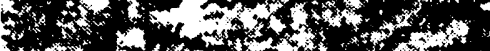

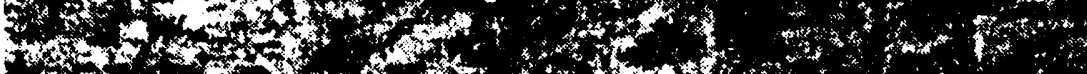

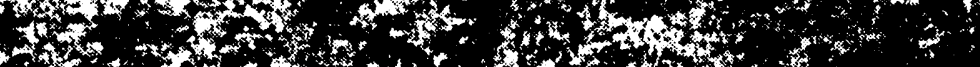

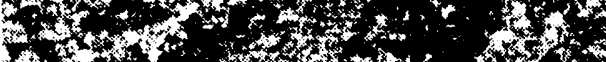

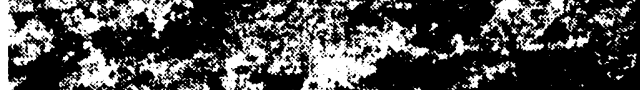

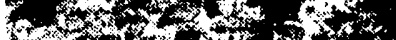

1.

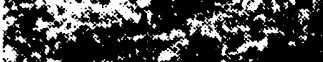

sin

at. 
WHC-EP-0625

This page intentionally left blank. 


\section{Tank 241-C-109}

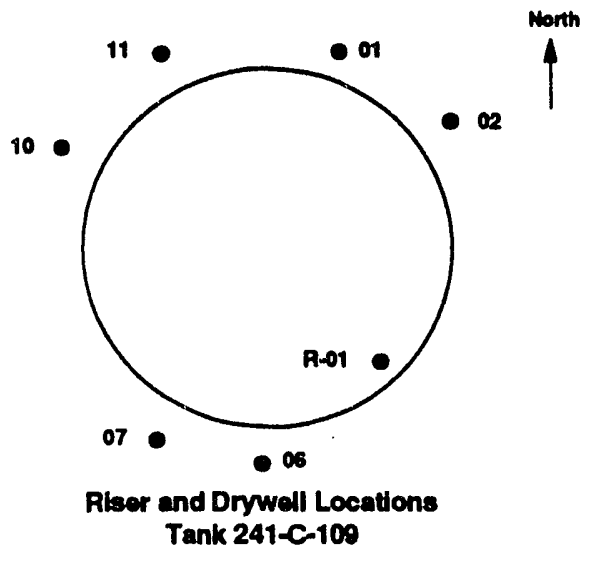

Number of External Drywells: 6

Number of Lateral Wells: None

\section{Tank Status}

Watch List: Ferrocyanide

Contents

Type: Non-Complexed Waste

Total Waste: $66 \mathrm{~K}$ gallons

Supernate Volume: $4 \mathrm{~K}$ gallons

Drainable Interstitial Liquid: OK gallons

Isolation Status

Date Interim Stabilized: $11 / 29 / 83$

Date Interim Isolated: $12 / 15 / 82$

Surface Level/Leak Status

Integrity Category: Sound

Manual Tape Surface Level: 18.00 Inches (11/23/92)

Last Photographed: $01 / 30 / 76$

Photo Interpretation Comments: See attached photo.

Temperature Status

Highest temperature during 1992: $88^{\circ} \mathrm{F}(09 / 30 / 92)$

Comments: Temperatures are stable.

Drywell Status

Comments: Current drywell profiles were stable and consistent with established baseline profiles.
Tank Description

Type: Single Shell

Constructed: 1944

In-service: $04 / 48$

Out of Service: 1976

Diameter: 75 '

Usable Depth: 16'

Capacity: 530K gallons

Bottom shape: Dish

Hanford Coordinates:

$43,003^{\prime}$ North

48,327' West

Ventllation: Passive

\section{Leak Detection System}

Surface Level:

FIC Riser- None

Manual Tape Riser- R-01

LOW Riser(s)- None 
WHC-EP-0625

This page intentionally left blank. 




Tank: 241-C-109

August 1986 
WHC-EP-0625

This page intentionally left blank. 


\section{Tank 241-C-110}

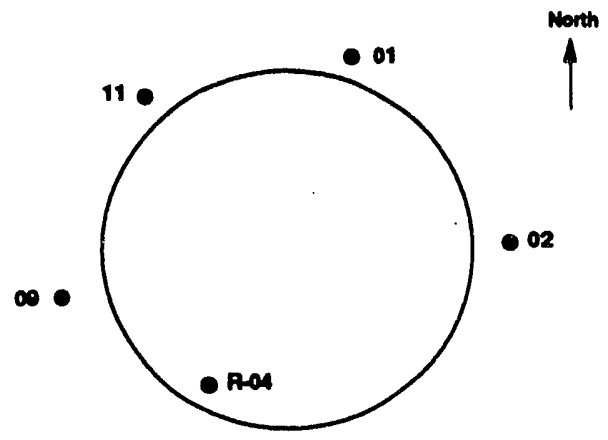

Rlaer and Drywdl Locationa Tank 241-C-110

Number of External Drywells: 4

Number of Lateral Wells: None

\section{Tank Status}

Contents

Type: Dilute Complexed Waste

Total Waste: $187 \mathrm{~K}$ gallons

Supernate Volume: OK gallons

Drainable Interstitial Liquid: $7 \mathrm{~K}$ gallons

Isolation Status

Date Interim Stabilized: 09/79

Date Partially Interim Isolated: $12 / 15 / 82$

\section{Surface Level/Leak Status}

Integrity Category: Assumed Leaker

Date Declared Confimed or Assumed Leaker: 1984

Leak Volume Estumate: 2,000 Gallons (1989)

Manual Tape Surface Level: 65.00 Inches (12/28/92)

Last Photographed: $08 / 12 / 86$

Photo Interpretation Comments:

Photographs contunue to show more surface liquid on one side of the tank than prevtous photographs and large areas of exposed solids in the remaining areas of the tank. The manual tape donought plummet is contacting a shallow clear liquid surface.

Temperature Status

Highest temperature during 1992: $70.5^{\circ} \mathrm{F}(07 / 02 / 92)$

Comments: Temperatures are stable.

Drywell Status

Comments: Current drywell profiles were stable and consistent with established baseline profiles. 
WHC-EP-0625

This page intentionally left blank. 


\section{Tank 241-C-111}

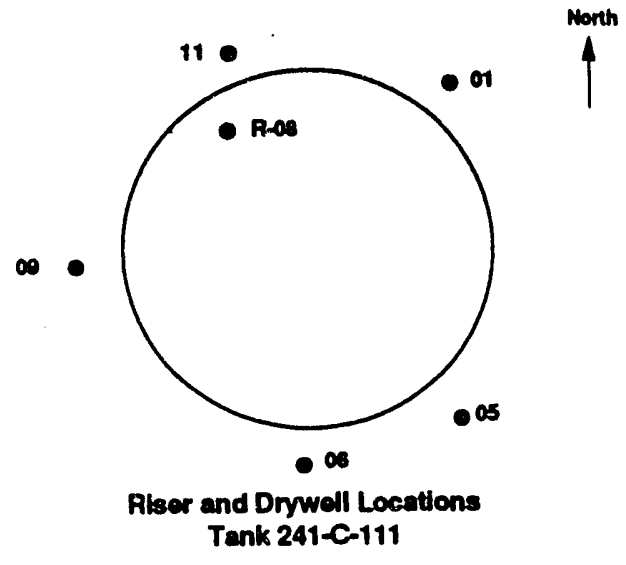

Number of External Drywells: 5 Number of Lateral Wells: None

\section{Tank Status}

Watch List: Ferrocyanide Conterits

Type: Non-Complexed Waste

Total Waste: $57 \mathrm{~K}$ gallons

Supernate Volume: OK gallons

Drainable Interstilial Liquid: OK gallons

Isolation Status

Date Interim Stabilized: 03/09/84

Date Interim Isolated: $12 / 15 / 82$

\section{Surface Level/Leak Status}

Integrity Category: Assumed Leaker

Date Declared Confirmed or Assumed Leaker: 1968

Leak Volume Estimate: 5,500 Gallons (1989)

Manual Tape Surface Level: 16.25 Inzhes (11/23/92)

Last Photographed: $02 / 25 / 70$

Photo Interpretation Comments: See attached photo.

Temperature Status

Highest temperature during 1992: $82.2{ }^{\circ} \mathrm{F}(10 / 02 / 92)$

Comments: Temperatures are stable.

\section{Drywell Status}

Comments: Current drywell profiles were stable and consistent with established baseline profiles.
Tank Description

Type: Single Shell

Constructed: 1944

Out of Service: 1968

Diameter. $75^{\circ}$

Usable Depth: $16^{\prime}$

Capacity: $530 \mathrm{~K}$ gallons

Bottom shape: Dish

Hanford Coordinates: 43,003' North 48,469' West

Ventilation: Passive

\section{Leak Detection System}

Surface Level:

FIC Riser- None

Manual Tape Riser- R-08

LOW Riser(s)- None 


\section{WHC-EP-0625}

This page intentionally left blank. 
WHC-EP-0625

\section{Tank: 241-C-111}

\section{February 1970}
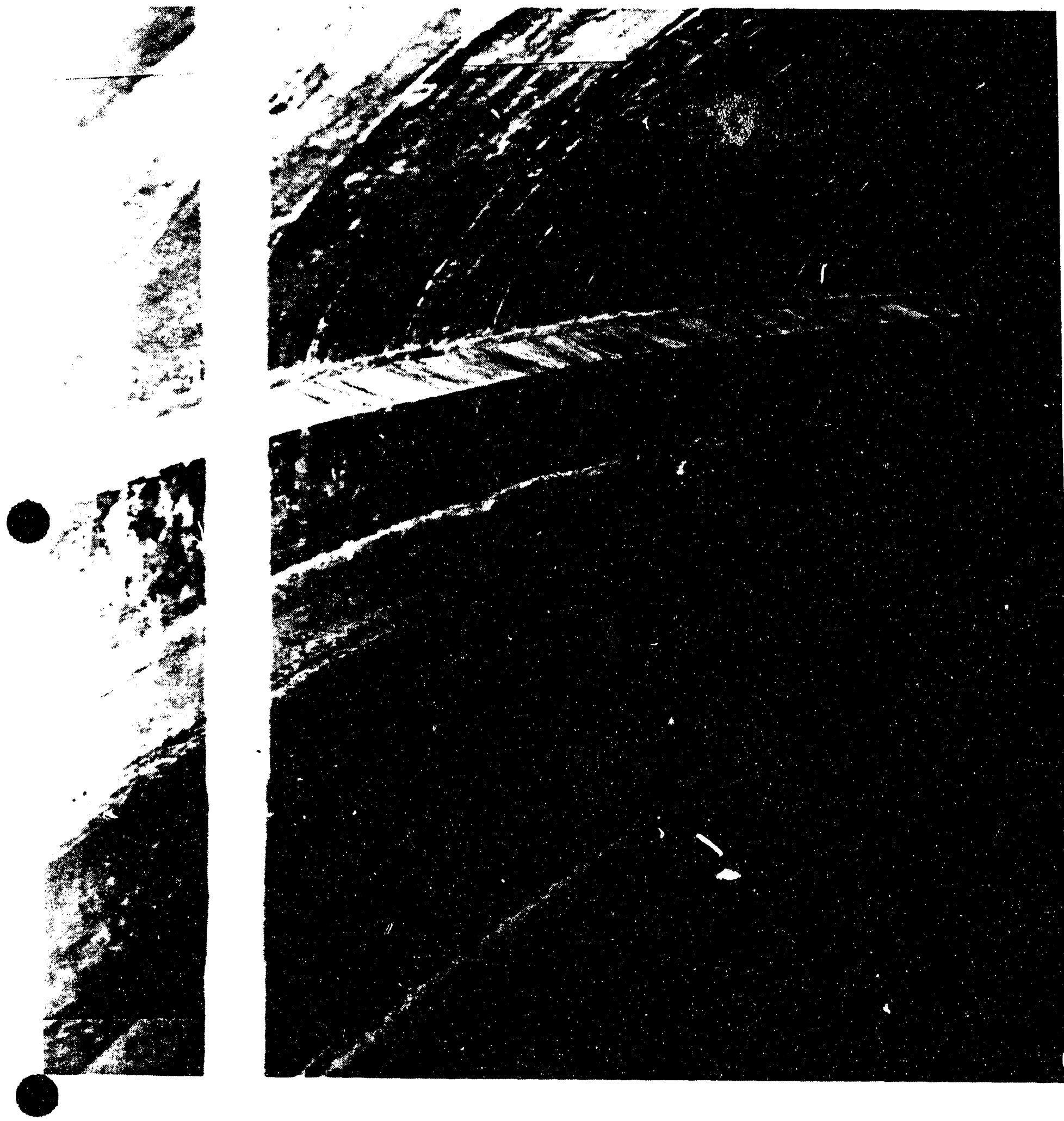
WHC-EP-0625

This page intentionally left blank. 


\section{Tank 241-C-112}

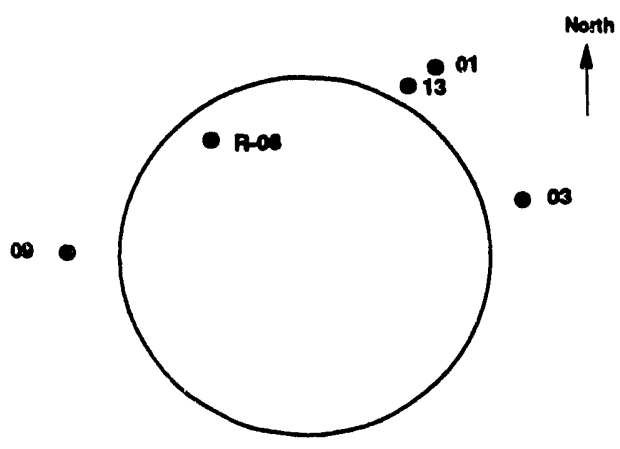

Plser and Drywell Locations Tank 241-C-112

Number of External Drywells: 4

Number of Lateral Wells: None

\section{Tank Status}

Watch List: Ferrocyanide

Contents

Type: Non-Complexed Waste

Total Waste: $104 \mathrm{~K}$ gallons

Supernate Volume: OK gallons

Drainable Interstitial Liquid: $32 \mathrm{~K}$ gallons

Isolation Status

Date Partially Interim Isolated: 12/15/82

Date Interim Stabilized: $09 / 90$

Surface Level/Leak Status

Integrity Category: Sound

Manual Tape Surface Level: 33.50 Inches (12/28/92)

Last Photographed: 09/18/90

Temperature Status

Highest temperature during 1992: $90.50{ }^{\circ} \mathrm{F}(08 / 09 / 92)$

Comments: Temperatures are stable.

Drywell Status

Comments: Current drywell profiles were stable and consistent with established baseline profiles. 
WHC-EP-0625

This page intentionally left blank. 
Whe th it:-

Tank: 241-C-112

July 1990

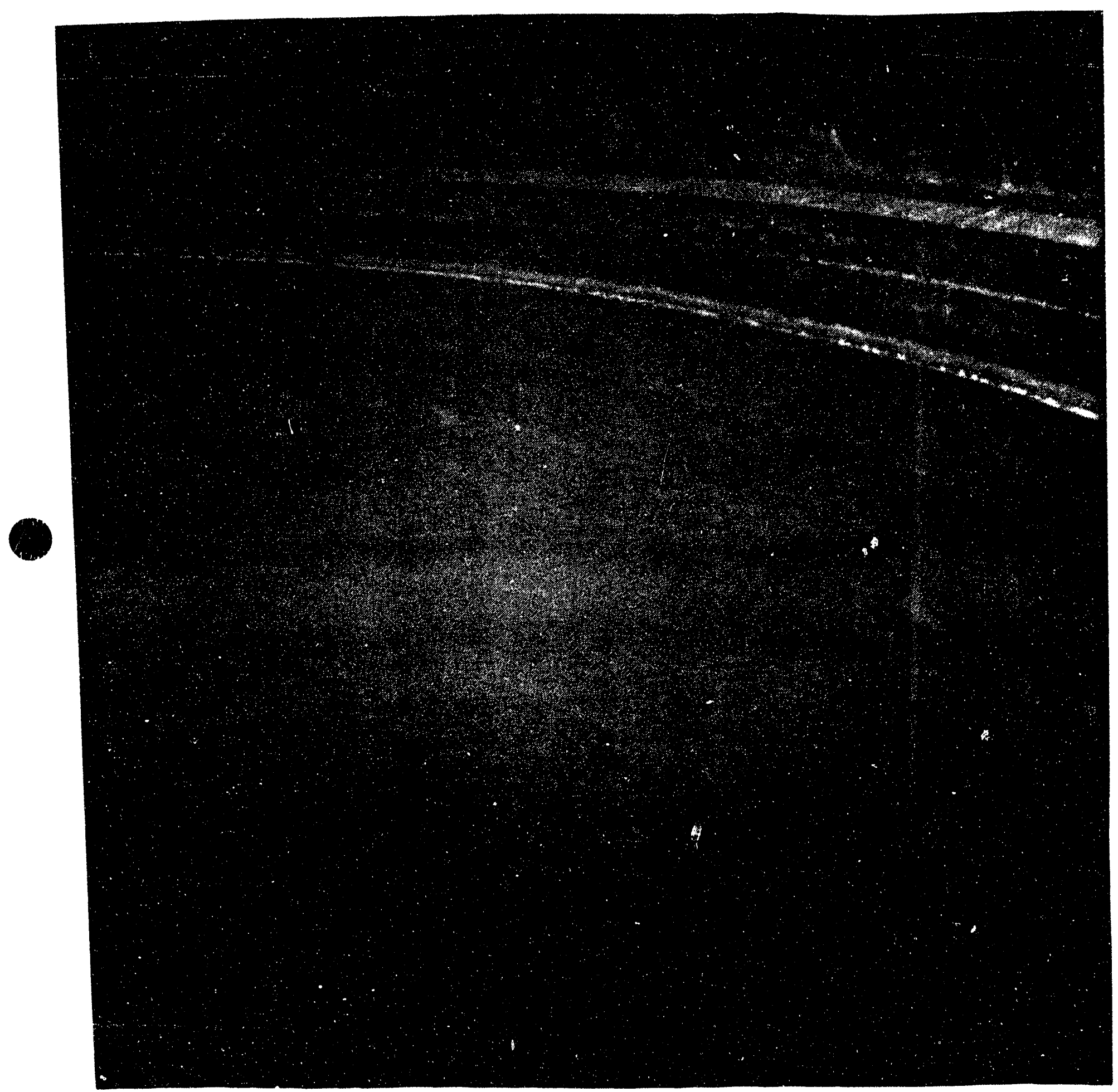

$90071234.6 C^{A}$ 
WHC-EP-0625

This page intentionally left blank. 


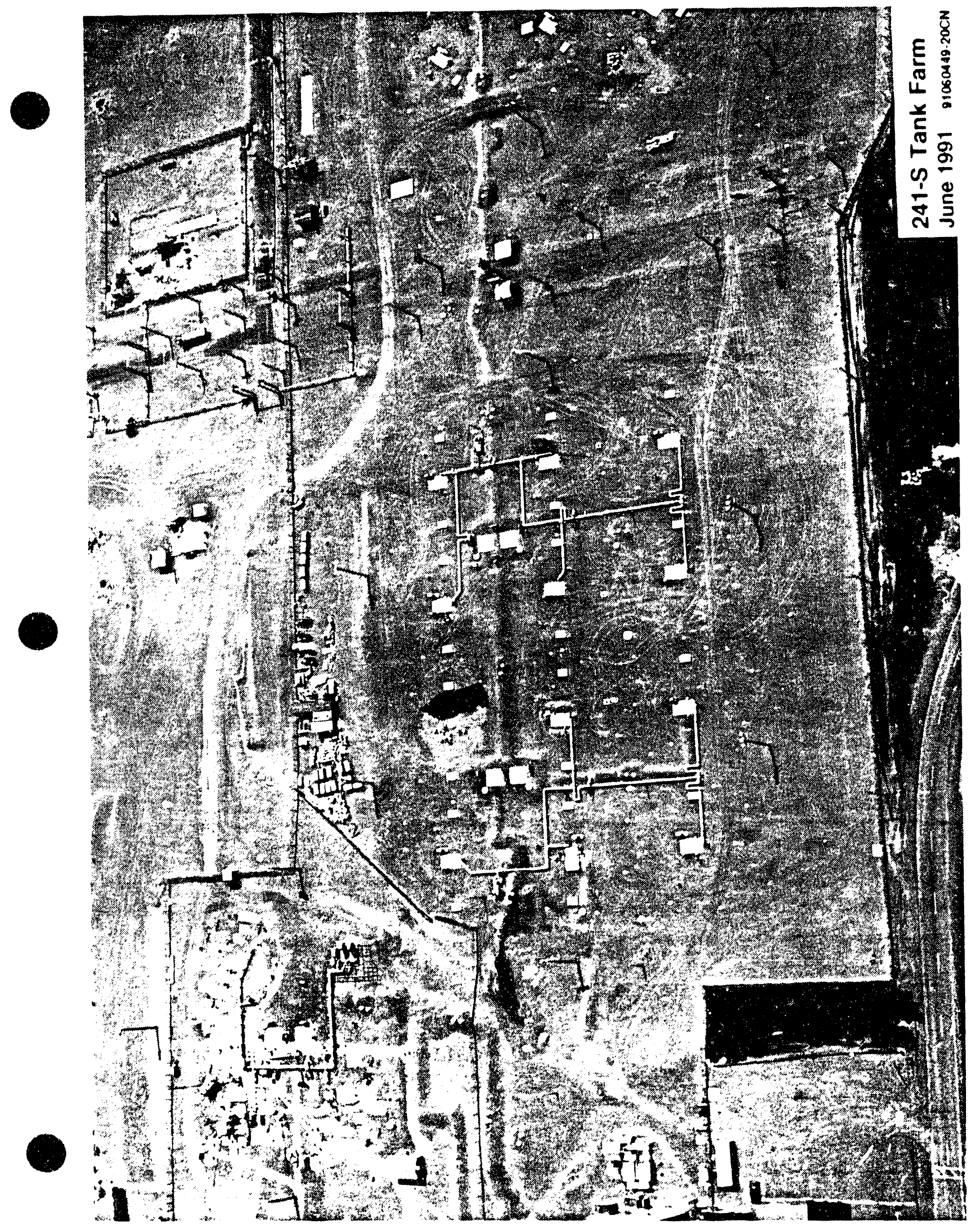


WHC-EP-0625

This page intentionally left blank. 


\section{Tank 241-S-101}

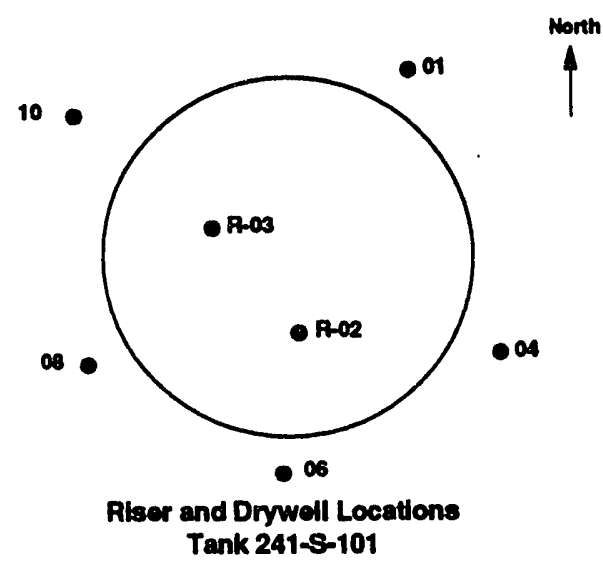

Number of External Drywells: 5

Number of Lateral Wells: None

\section{Tank Status}

Contents

Type: Non-Complexed Waste

Total Waste: $427 \mathrm{~K}$ gallons

Supernate Volume: $12 \mathrm{~K}$ gallons

Drainable Interstitial Liquid: $84 \mathrm{~K}$ gallons

Isolation Status

Date Partially Interim Isolated: $12 / 15 / 82$

Surface Level/Leak Status

Integrity Category: Sound

FIC Surface Level: 161.10 Inches (12/21/92)

Last Photographed: $03 / 18 / 88$

Photo Interpretation Comments: See attached photo.

Temperature Status

Highest temperature during 1992: $123^{\circ} \mathrm{F}(11 / 07 / 92)$

Comments: Temperatures are stable.

\section{Drywell Status}

Comments: Current drywell profles were stable and consistent with established baseline profiles.
Tank Description

Type: Single Shell

Constructed: 1951

In-service: $07 / 17 / 53$

Dlameter: $\mathbf{7 5}^{\prime}$

Usable Depth: 23'

Capacity: $758 \mathrm{~K}$ gallons

Bottom shape: Dish

Hanford Coordinates:

$36,226^{\prime}$ North

$75,665^{\circ}$ ' West

Ventilation: Passive

\section{Leak Detection System}

Surface Level:

Manual Tape Riser- None

LOW Riser(s)- R-02
FIC Riser- R-03 


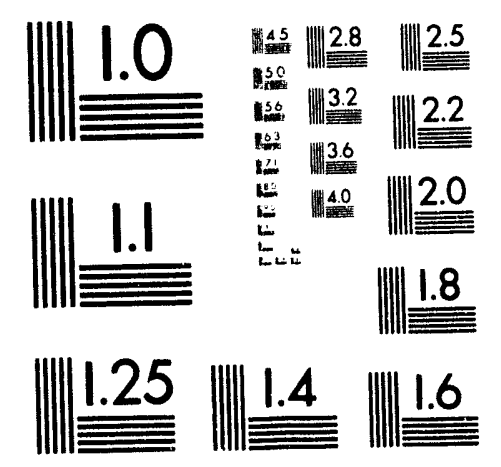



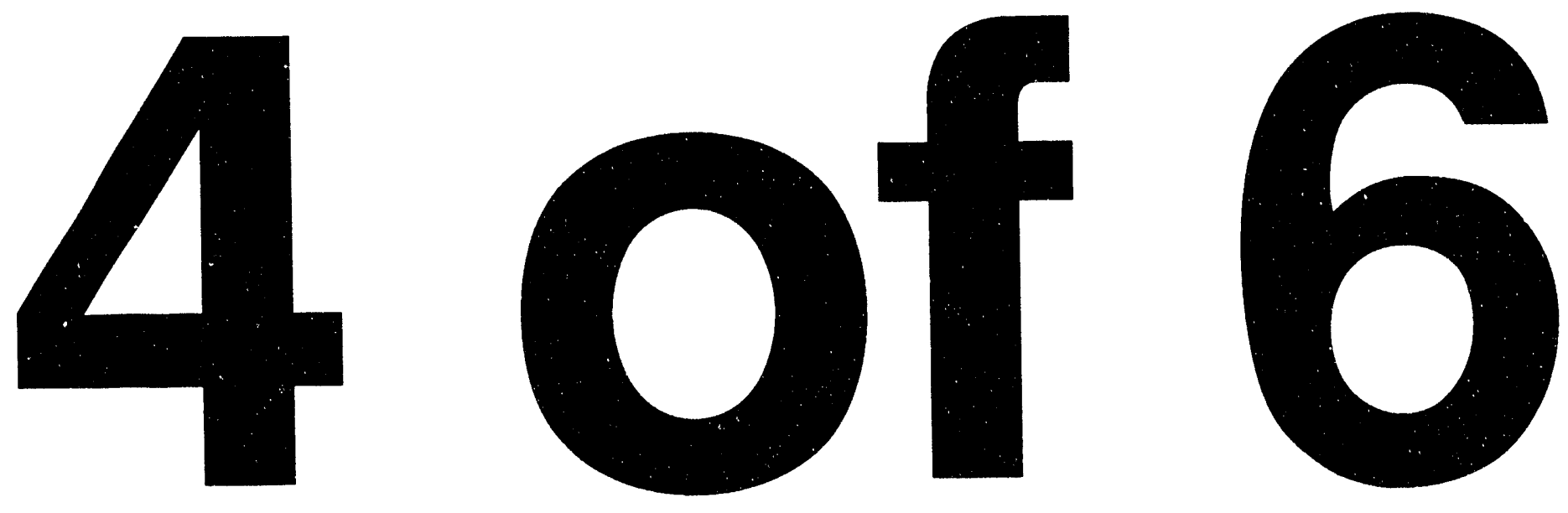


\section{WHC-EP-0625}

This page intentionally left blank. 


\section{Tank 241-S-102}

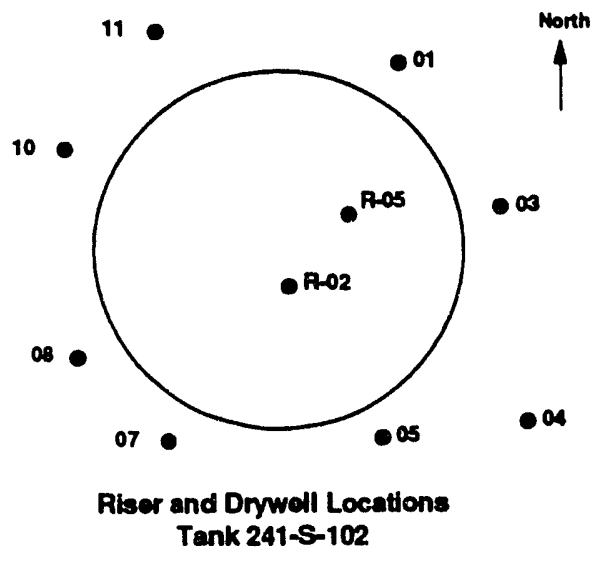

Number of External Drywells: 8

Number of Lateral Wells: None

\section{Tank Status}

Watch List: Hydrogen, Organic Salts

Contents

Type: Double Shell Slurry Feed

Total Waste: $549 \mathrm{~K}$ gallons

Supernate Volume: OK gallons

Drainable Interstitial Liquid: $230 \mathrm{~K}$ gallons

Isolation Status

Date Partially Interim Isolated: $12 / 15 / 82$

Surface Level/Leak Status

Integrity Category: Sound

FIC Surface Level: 204.00 Inches (12/28/92)

Last Photographed: 03/18/88

Photo Interpretation Comments: See attached photo.

Temperature Status

Highest temperature during 1992: $109.7^{\circ} \mathrm{F}(01 / 12 / 92)$

Comments: Temperatures are stable.

\section{Drywell Status}

Comments: Current drywell profiles were stable and consistent with established baseline profiles.
Tank Description

Type: Single Shell

Constructed: 1951

In-service: 1953

Diameter: $7^{\circ}$

Usable Depth: 23'

Capacity: $758 \mathrm{~K}$ gallons

Bottom shape: Dish

Hanford Coordinates: $36,226^{\prime}$ North

75.767' West

Ventllation: Passive

\section{Leak Detection System \\ Surface Level: \\ FIC Riser- R-02 \\ Manual Tape Riser- None \\ LOW Riser(s)- R-05}


WHC-EP-0625

This page intentionally left blank. 


\title{
Tank: 241-S-102
}

\author{
Marm 1988
}


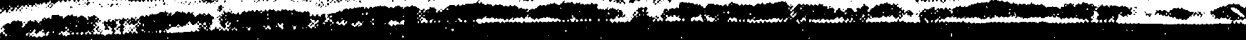

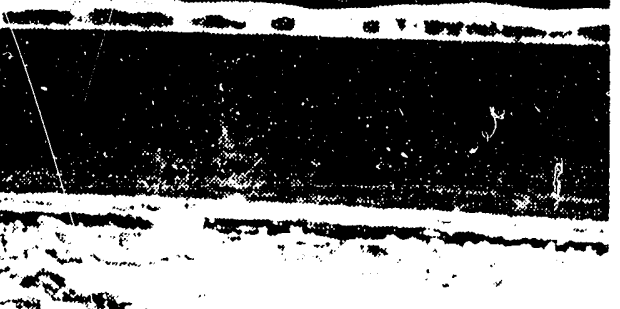

46 \%

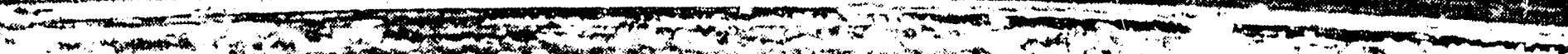

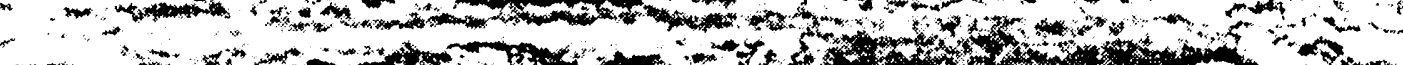
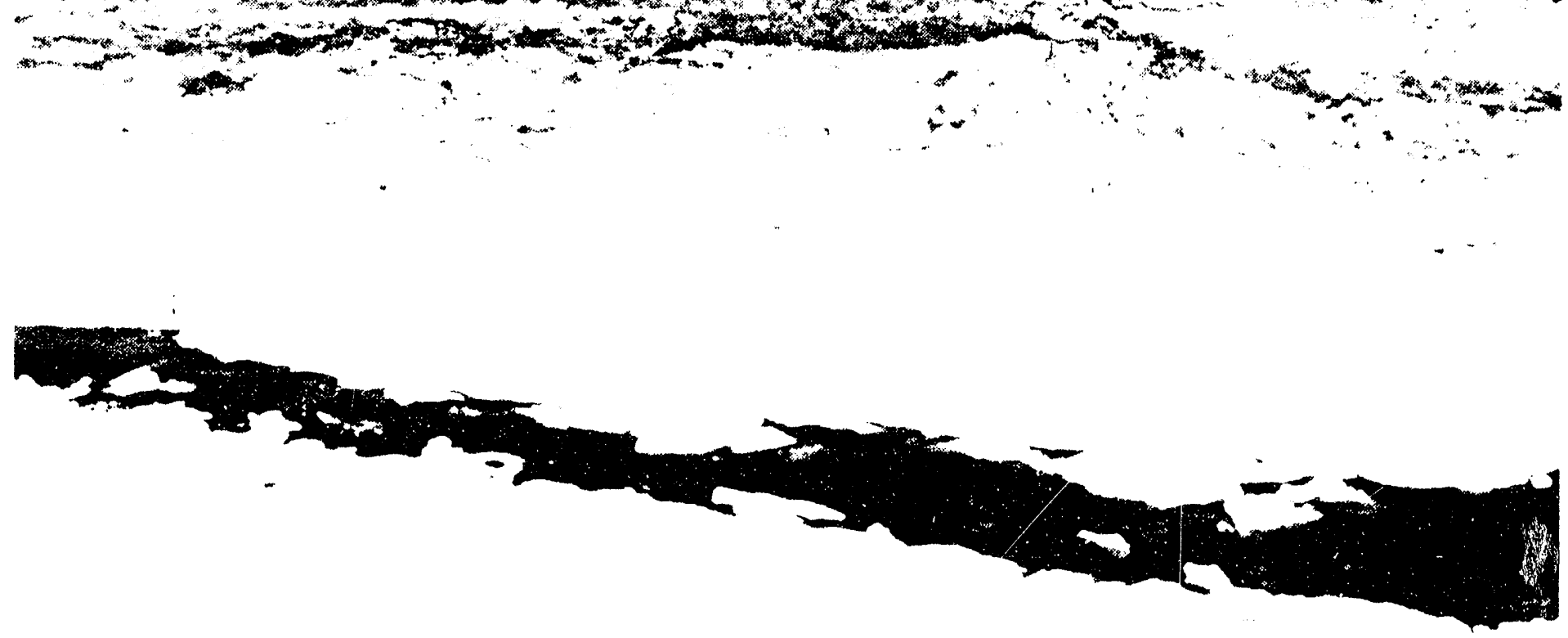


\section{WHC-EP-0625}

This page intentionally left blank. 


\section{Tank 241-S-103}

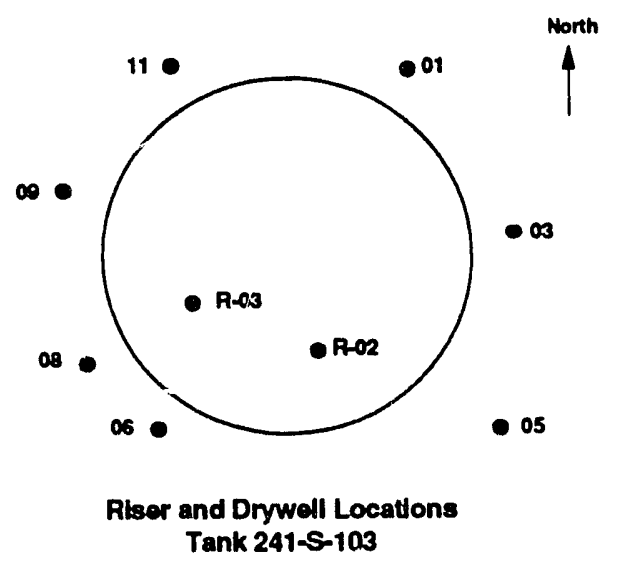

Number of External Drywells: 7

Number of Lateral Wells: None

\section{Tank Status}

Contents

Type: Double Shell Slurry Feed

Total Waste: $248 \mathrm{~K}$ gallons

Supernate Volume: $17 \mathrm{~K}$ gallons

Drainable Interstitial Liquid: $85 \mathrm{~K}$ gallons

Isolation Status

Date Partially Interim Isolated: 12/15/82

\section{Surface Level/Leak Status}

Integrity Category: Sound

FIC Surface Level: 103.65 Inches (12/28/92)

Last Photographed: 06/01/89

Photo Interpretation Comments:

Photographs show a liquid surface with solids visible beneath the surface and large areas (some floating) of exposed solids. The FIC plummet appears to be contacting soft solids, but is very difficult to see.

Temperature Status

Highest temperature during 1992: $91.2{ }^{\circ} \mathrm{F}(01 / 01 / 92)$

Comments: Temperatures are stable.

Drywell Status

Comments: Current drywell profiles were stable and consistent with established baseline profiles.
Tank Description

Type: Single Shell

Constructed: 1951

In-service: $11 / 01 / 53$

Diameter: $7^{\circ}$

Usable Depth: 23'

Bottom shape: Dish

Hanford Coordinates: $36,226^{\prime}$ North 75.869' West

Ventilation: Passive

\section{Leak Detection System}

Surface Level:

FIC Riser- R-03

Manual Tape Riser- None

LOW Riser(s)- R-02
Capacity: $758 \mathrm{~K}$ gallons 
WHC-EP-0625

This page intentionally left blank. 


\section{Tank 241-S-104}

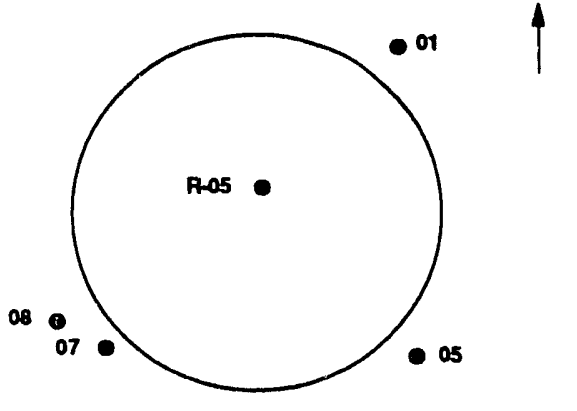

Riser and Drywell Locations Tank 241-S-104

Number of External Drywells: 4

Number of Lateral Wells: None

\section{Tank Status \\ Contents}

Type: Non-Complexed Waste

Total Waste: $294 \mathrm{~K}$ gallons

Supernate Volume: $1 \mathrm{~K}$ gallons

Drainable Interstitial Liquid: $28 \mathrm{~K}$ gallons

Isolation Status

Date Interim Stabilized: 12/20/83

\section{Surface Level/Leak Status}

Integrity Category: Assumed Leaker

Date Declared Confirmed or Assumed Leaker: 1968

Leak Volume Estimate: 24,000 Gallons (1989)

Manual Tape Surface Level: 113.50 Inches (12/28/92)

Last Photographed: $12 / 12 / 84$

Photo Interpretation Comments:

Photographs show a smooth surface of sludge with very shallow liquid covering portions of the solids, and a shallow liquid pool around the salt well.

Temperature Status

Highest temperature during 1992: $112.3^{\circ} \mathrm{F}(01 / 01 / 92)$

Comments: Temperatures are stable.

\section{Drywell Status}

Comments: Current drywell profiles were stable and consistent with established baseline proflles. 
WHC-EP-0625

This page intentionally left blank. 


\section{Tank 241-S-105}

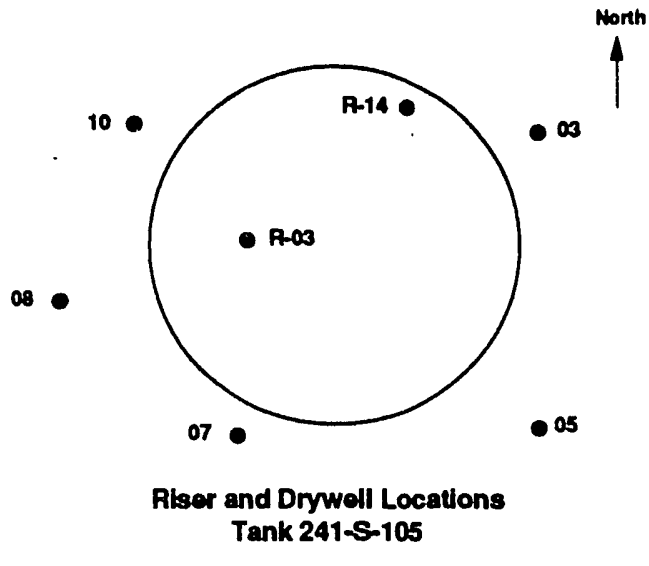

Number of External Drywells: 5 Number of Lateral Wells: None

\section{Tank Status}

Contents

Type: Non-Complexed Waste

Total Waste: $456 \mathrm{~K}$ gallons

Supernate Volume: OK gallons

Drainable Interstitial Liquid: $35 \mathrm{~K}$ gallons

Isolation Status

Date Partially Interim Isolated: $12 / 15 / 82$

Date Interim Stabilized: 09/88

\section{Surface Level/Leak Status}

Integrity Category: . Sound

FIC Surface Level: 161.60 Inches (12/28/92)

Surface Level Comments: Intrusion Mode

Last Photographed: 04/12/89

Photo Interpretation Comments:

Photographs show an uneven heavily crusted surface of saltcake and a pool of liquid around the salt well. The FIC plummet is contacting solids in a hole and

Temperature Status measurement anomalies can be expected.

Highest temperature during 1992: $79.1^{\circ} \mathrm{F}(01 / 01 / 92)$

Comments: Temperatures are stable.

\section{Drywell Status}

Comments: Current drywell profiles were stable and consistent with establishec. baseline profiles.
Tank Description

Type: Single Shell

Constructed: 1951

In-service: 1953

Out of Service: 1974

Diameter: 75'

Usable Depth: 23'

Capacity: $758 \mathrm{~K}$ gallons

Bottom shape: Dish

Hanford Coordinates:

$36,124^{\circ}$ North

75.767' West

Ventilation: Passive

\section{Leak Detection System}

Surface Level:

FIC Riser- R-03

Manual Tape Riser- None

LOW Riser(s)- R-14 
WHC-EP-0625

This page intentionally left blank. 


\section{Tank 241-S-106}

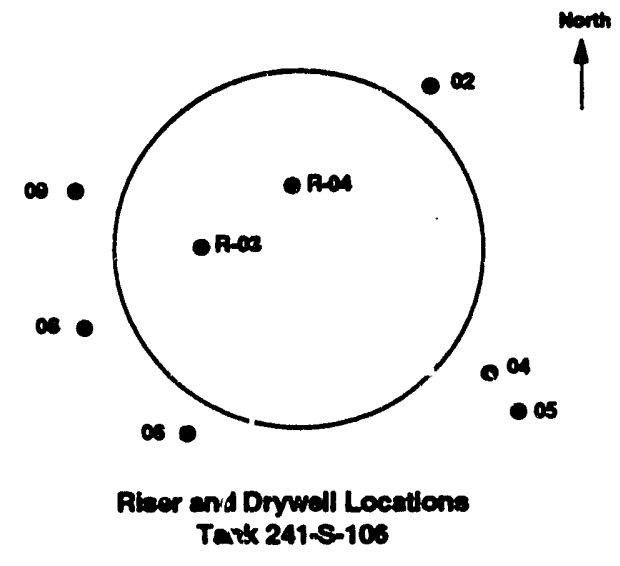

Number of External Drywells: 6 Number of Lateral Wells: None

\section{Tank Status \\ Contents}

Type: Non-Corr slexed Waste

Total Waste: $543 \mathrm{~K}$ gallons

Supernate Volume: OK gallons

Drainable Interstitial Liquid: $185 \mathrm{~K}$ gallons

\section{Isolation Status}

Date Partially Interim Isolated: $12 / 15 / 82$

Surface Level/Leak Status

Integrity Category: Sound

FIC Surface Level: 177.90 Inches (12/28/92)

Surface Level Comments: Intrusion Mode

Last Photographed: 03/17/89

Photo Interpretation Comments:

Photographs show a cracked uneven surface of salt cake with a pool of liquid under the FIC plummet and also around the salt well and in-tank dry well casing. There appears to be more liquid than in previous photos. although some sluffing of solids is evident.

\section{Temperature Status}

Highest temperature during 1992: $93^{\circ} \mathrm{F}(07 / 03 / 92)$

Comments: Temperatures are stable.

\section{Drywell Status}

Comments: Current drywell profiles were stable and consistent with established baseline proflles.
Tank Description

Type: Single Shell

Constructed: 1951

In-service: 1953

Diameter: $7^{\circ}$

Usable Depth: 23'

Capacity: 758K gallons

Bottom shape: Dish

Hanford Coordinates:

$36,124^{\circ}$ North

75,869' West

Ventilation: Passive

\section{Leak Detection System}

Surface Level:

Manual Tape Riser- None

LOW Riser(s)- R-04
FIC Riser- R-03 
WHC-EP-0625

This page intentionally left blank. 


\section{Tank 241-S-107}

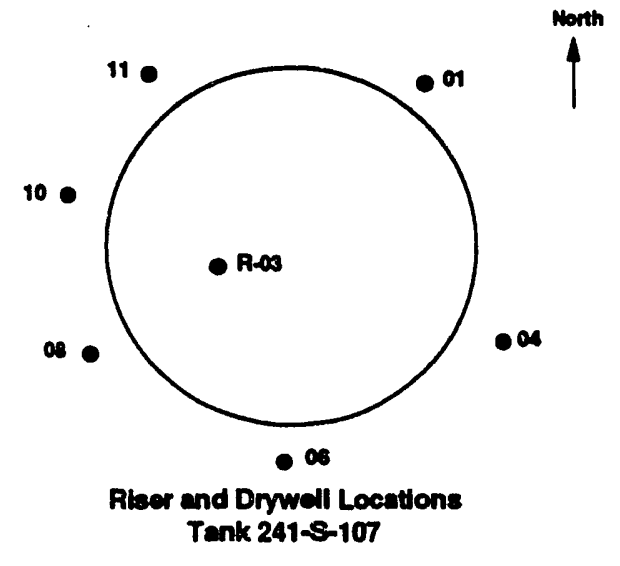

Number of External Drywells: 6

Number of Lateral Wells: None

\section{Tank Status}

Contents

Type: Non-Complexed Waste

Total Waste: $368 \mathrm{~K}$ gallons

Supernate Volume: $6 \mathrm{~K}$ gallons

Drainable Interstitial Liquid: $45 \mathrm{~K}$ gallons

Isolation Status

Date Partially Interim Isolated: 12/15/82

\section{Surface Level/Leak Status}

Integrity Category: Sound

FIC Surface Level: 144.30 Inches (12/28/92)

Last Photographed: 03/12/87

Photo Interpretation Comments:

Photographs reveal a large area of clear liquid toward the center of the tank and exposed solids around the perimeter. The FIC plummet is contacting liquid. No photograph comparisons were made (active to 10/80).

\section{Temperature Status}

Highest temperature during 1992: $113^{\circ} \mathrm{F}(01 / 01 / 92)$

Comments: Temperatures are stable.

\section{Drywell Status}

Comments: Current drywell profiles were stable and consistent with established baseline profiles.
Tank Description

Type: Single Shell

Constructed: 1951

In-service: $08 / 25 / 52$

Diameter: $7^{\circ}$

Usable Depth: 23'

Capacity: $758 \mathrm{~K}$ gallons

Bottom shape: Dish

Hanford Coordinates:

36,022 ' North

75,665' West

Ventllation: Passive

\section{Leak Detection System}

Surface Level:

FIC Riser- R-03

Manual Tape Riser- None

LOW Riser(s)- None 


\section{WHC-EP-0625}

This page intentionally left blank. 


\section{Tank 241-S-108}

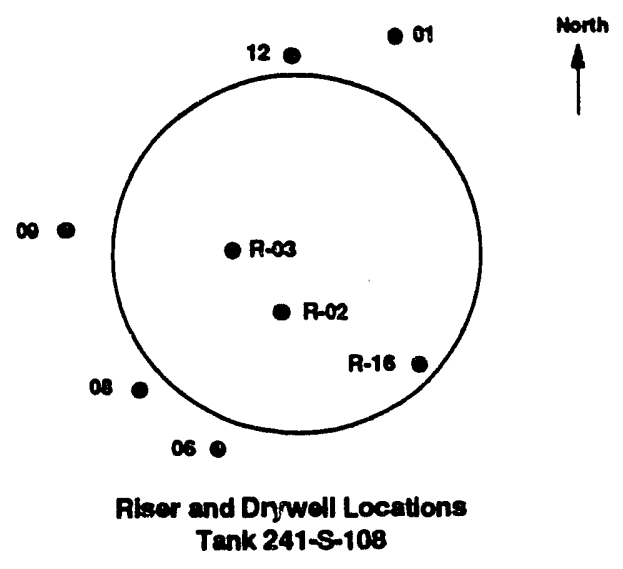

Number of External Drywells: 5

Number of Lateral Wells: None
Tank Description

Type: Single Shell

Constructed: 1951

In-service: $10 / 30 / 52$

Out of Service: 1979

Diameter: 75'

Usable Depth: 23'

Capacity: $758 \mathrm{~K}$ gallons

Bottom shape: Dish

Hanford Coordinates:

36,022 ' North

75,767' West

Ventilation: Passive

\section{Leak Detection System}

Surface Level:

FIC Riser- R-03

Manual Tape Riser- R-02

LOW Riser(s)- R-16

\section{Tank Status}

Contents

Type: Non-Complexed Waste

Total Waste: $604 \mathrm{~K}$ gallons

Supernate Volume: OK gallons

Drainable Interstitial Liquid: $127 \mathrm{~K}$ gallons

Isolation Status

Date Interim Stabilized: 04/79

Date Partially Interim Isolated: 12/15/82

Surface Level/Leak Status

Integrity Category: Sound

FIC Surface Level: Intrusion level set at 172.80 Inches $(12 / 28 / 92)$

Surface Level Comments: Intrusion Mode

Last Photographed: 03/12/87

Photo Interpretation Comments:

Photographs show a dry cracked uneven surface. The FIC plummet is contacting solids in a hole (created by the plummet) on the slope of a depression in the salt cake.

\section{Temperature Status}

Highest temperature during 1992: $94^{\circ} \mathrm{F}(01 / 01 / 92)$

Comments: Temperatures are stable.

\section{Drywell Status}

Comments: Current drywell profiles were stable and consistent with established baseline profiles. 
WHC-EP-0625

This page intentionally left blank. 


\section{Tank 241-S-109}

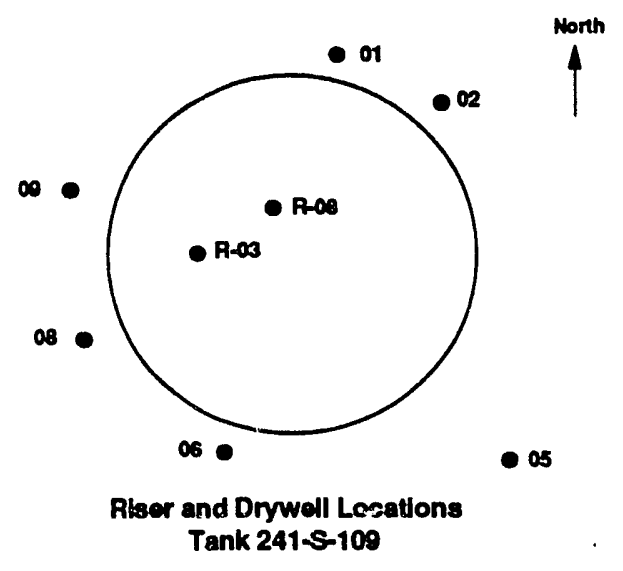

Tank Description

Type: Single Shell

Constructed: 1951

In-service: $12 / 23 / 52$

Diameter: $75^{\prime}$

Usable Depth: 23'

Capacity: $758 \mathrm{~K}$ gallons

Bottom shape: Dish

Hanford Coordinates:

$36,022^{\prime}$ North

75,869' West

Ventilation: Passive

\section{Leak Detection System}

Surface Level:

FIC Riser- R-03

Manual Tape Riser- None

LOW Riser(s)- R-08

Number of External Drywells: 6

Number of Lateral Wells: None

\section{Tank Status}

\section{Contents}

Type: Non-Complexed Waste

Total Waste: $568 \mathrm{~K}$ gallons

Supernate Volume: OK gallons

Drainable Interstitial Liquid: $141 \mathrm{~K}$ gallons

\section{Isolation Status}

Date Interim Stabilized: 05/79

Date Partially Interim Isolated: $12 / 15 / 82$

\section{Surface Level/Leak Status}

Integrity Category: Sound

FIC Surface Level: Intrusion level set at 173.80 Inches (12/28/92)

Surface Level Comments: Intrusion Mode

Last Photographed: 08/24/84

Photo Interpretation Comments:

Photographs show a dry cracked uneven surface of salt cake. The FIC plummet is in a large depression and measurement anomalies can be expected. There is now liquid visible around the salt well casing.

\section{Temperature Status}

Highest temperature during 1992: $150^{\circ} \mathrm{F}(10 / 16 / 92)$

Comments: Temperatures are stable.

\section{Drywell Status}

Comments: Current drywell profiles were stable and consistent with established baseline profiles. 
WHC-EP-0625

This page intentionally left blank. 


\section{Tank 241-S-110}

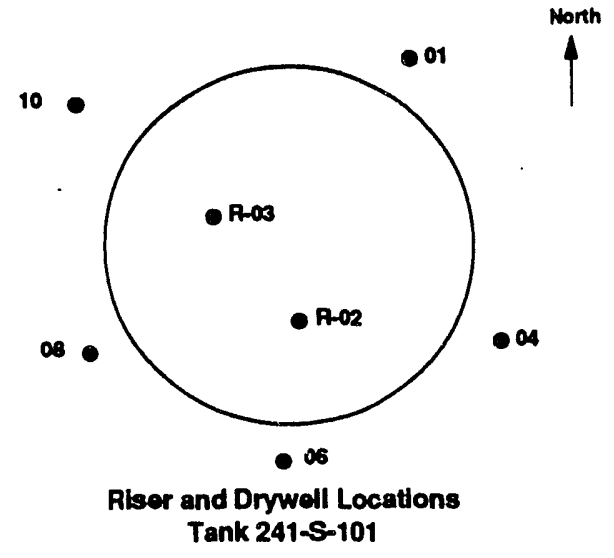

Number of External Drywells: 7

Number of Lateral Wells: None

\section{Tank Status}

Contents

Type: Non-Complexed Waste

Total Waste: $390 \mathrm{~K}$ gallons

Supernate Volume: OK gallons

Drainable Interstitial Liquid: $110 \mathrm{~K}$ gallons

Isolation Status

Date Interim Stabilized: 01/31/79

Date Partially Interim Isolated: $12 / 15 / 82$

Surface Level/Leak Status

Integrity Category: Sound

FIC Surface Level: Intrusion level set at 149.30 Inches $(12 / 28 / 92)$

Surface Level Comments: Intrusion Mode

Last Photographed: 03/12/87

Photo Interpretation Comments: No current photographs.

Temperature Status

Highest temperature during 1992: $121.50{ }^{\circ} \mathrm{F}(01 / 01 / 92)$

Comments: Temperatures are stable.

\section{Drywell Status}

Comments: Current drywell profiles were stable and consistent with established baseline profiles.
Tank Description

Type: Single Shell

Constructed: 1951

In-service: 1952

Out of Service: 1979

Diameter: $75^{\prime}$

Usable Depth: 23'

Capacity: $758 \mathrm{~K}$ gallons

Bottom shape: Dish

Hanford Coordinates: 35,920' North 75,665' West

Ventilation: Passive

\section{Leak Detection System \\ Surface Level:}

FIC Riser- R-03

Manual Tape Riser- None LOW Riser(s)- R-02 
WHC-EP-0625

This page intentionally left blank. 


\section{Tank 241-S-111}

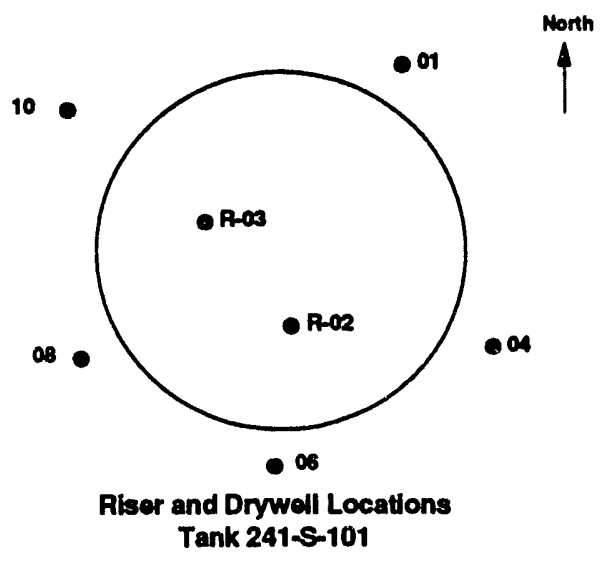

Number of External Drywells: 5

Number of Lateral Wells: None

\section{Tank Status}

\section{Watch List: Hydrogen}

\section{Contents}

Type: Non-Complexed Waste

Total Waste: $596 \mathrm{~K}$ gallons

Supernate Volume: 10K gallons

Drainable Interstitial Liquid: $195 \mathrm{~K}$ gallons

Isolation Status

Date Interim Stabllized: $03 / 78$

Date Partially Interim Isolated: 12/15/82

\section{Surface Level/Leak Status}

Integrity Category: Sound

FIC Surface Level: 203.40 Inches (12/28/92)

Last Photographed: 08/10/89

Photo Interpretation Comments: See attached photo.

Temperature Status

Highest temperature during 1992: $97^{\circ} \mathrm{F}(01 / 05 / 92)$

Comments: Temperatures are stable.

\section{Drywell Status}

Comments: Current drywell profles were stable and consistent with established baseline proflles.
Tank Description

Type: Single Shell

Constructed: 1951

In-service: 1952

Dlameter: $7^{\circ}$

Usable Depth: 23'

Capacity: $758 \mathrm{~K}$ gallons

Bottom shape: Dish

35,920' North

$75,767^{\circ}$ West

Ventilation: Passive

\section{Leak Detection System}

Surface Level:

FIC Riser- R-03

Manual Tape Riser- None

LOW Riser(s)- R-16
Hanford Coordinates: 
WHC-EP-0625

This page intentionally left blank. 



Tank: 241-S-111

Auqust 1989 


\section{WHC-EP-0625}

This page intentionally left blank. 


\section{Tank 241-S-112}

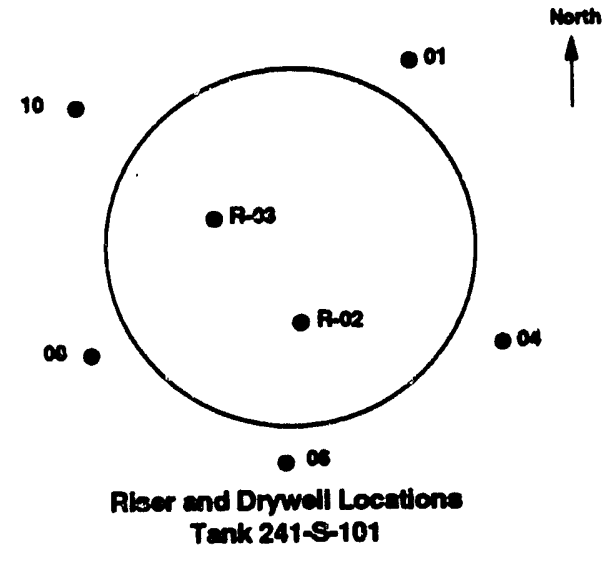

Number of External Drywells: 5

Number of Lateral Wells: None

\section{Tank Status}

\section{Watch List: Hydrogen}

\section{Contents}

Type: Non-Complexed Waste

Total Waste: $637 \mathrm{~K}$ gallons

Supernate Volume: OK gallons

Drainable Interstitial Liquid: $134 \mathrm{~K}$ gallons

Isolation Status

Date Interim Stabilized: 06/79

Date Partially Interim: Isolated: 12/15/82

Surface Level/Leak Status

Integrity Category: Saund

FIC Surface Level: Intrusion level set at 196.40 Inches (12/24/92)

Surface Level Comments: Intrusion Mode

Last Photographed: $03 / 24 / 87$

Photo Interpretation Comments: See attached photo.

Temperature Status

Highest iemperature during 1992: $92{ }^{\circ} \mathrm{F}(03 / 26 / 92)$

Comments: Temperatures are stable.

\section{Drywell Status}

Comments: Current drywell profiles were stable and consistent with established baseline profiles.
Tank Description

Type: Single Shell

Constructed: 1951

In-service: $07 / 25 / 52$

Diameter: $7^{\circ}$

Usable Depth: 23'

Capacity: $758 \mathrm{~K}$ gallons

Bottom shape: Dish

Hanford Coordinates:

35,920' North

75,869' West

Ventilation: Passive

\section{Leak Detection System \\ Surface Level:}

FIC Riser- R-03

Manual Tape Riser- None

LOW Riser(s)- R-08 
WHC-EP-0625

This page intentionally left blank. 
Tank: 241-S-112

March 198 ;
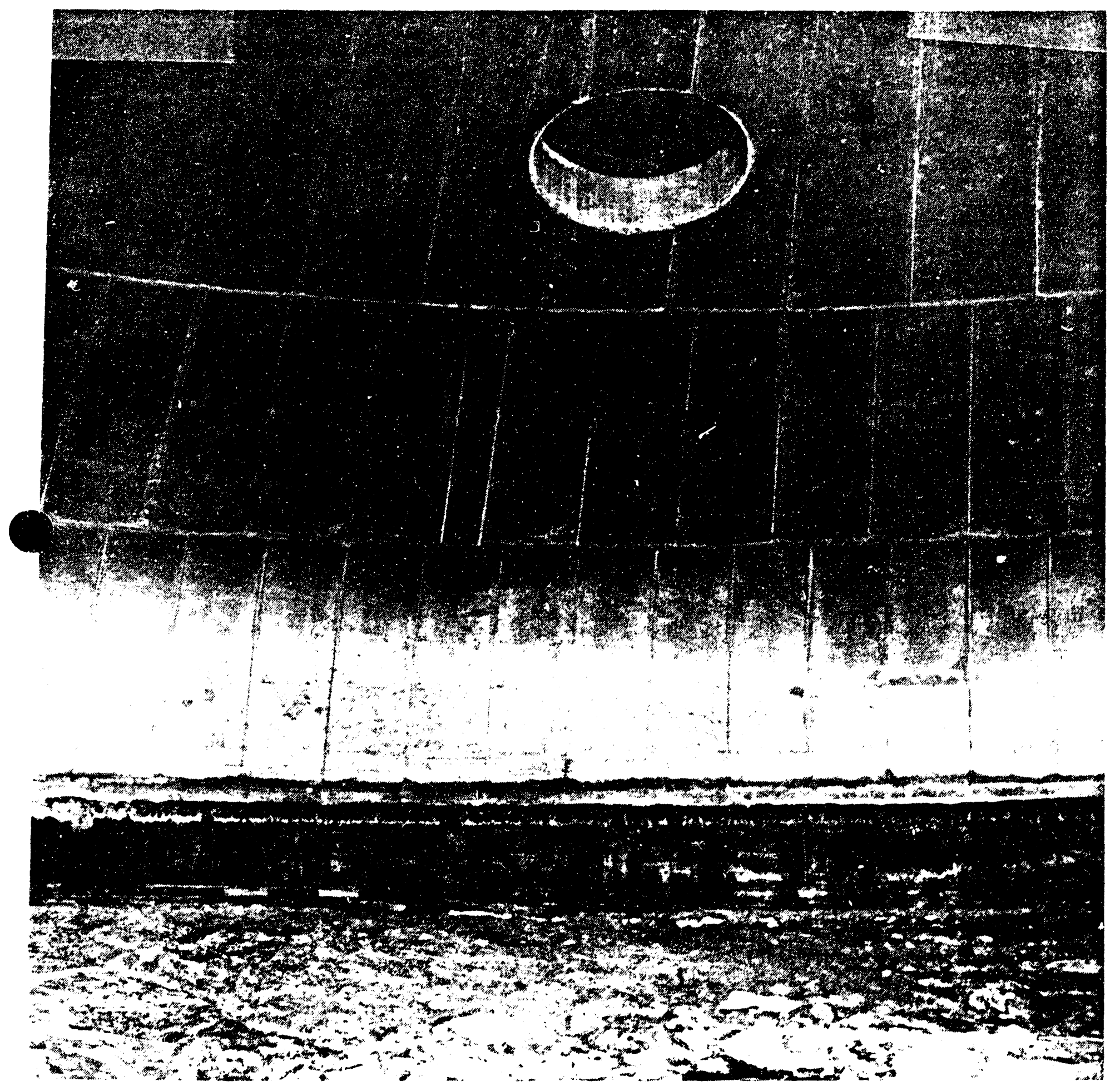
WHC-EP-0625

This page intentionally left blank. 


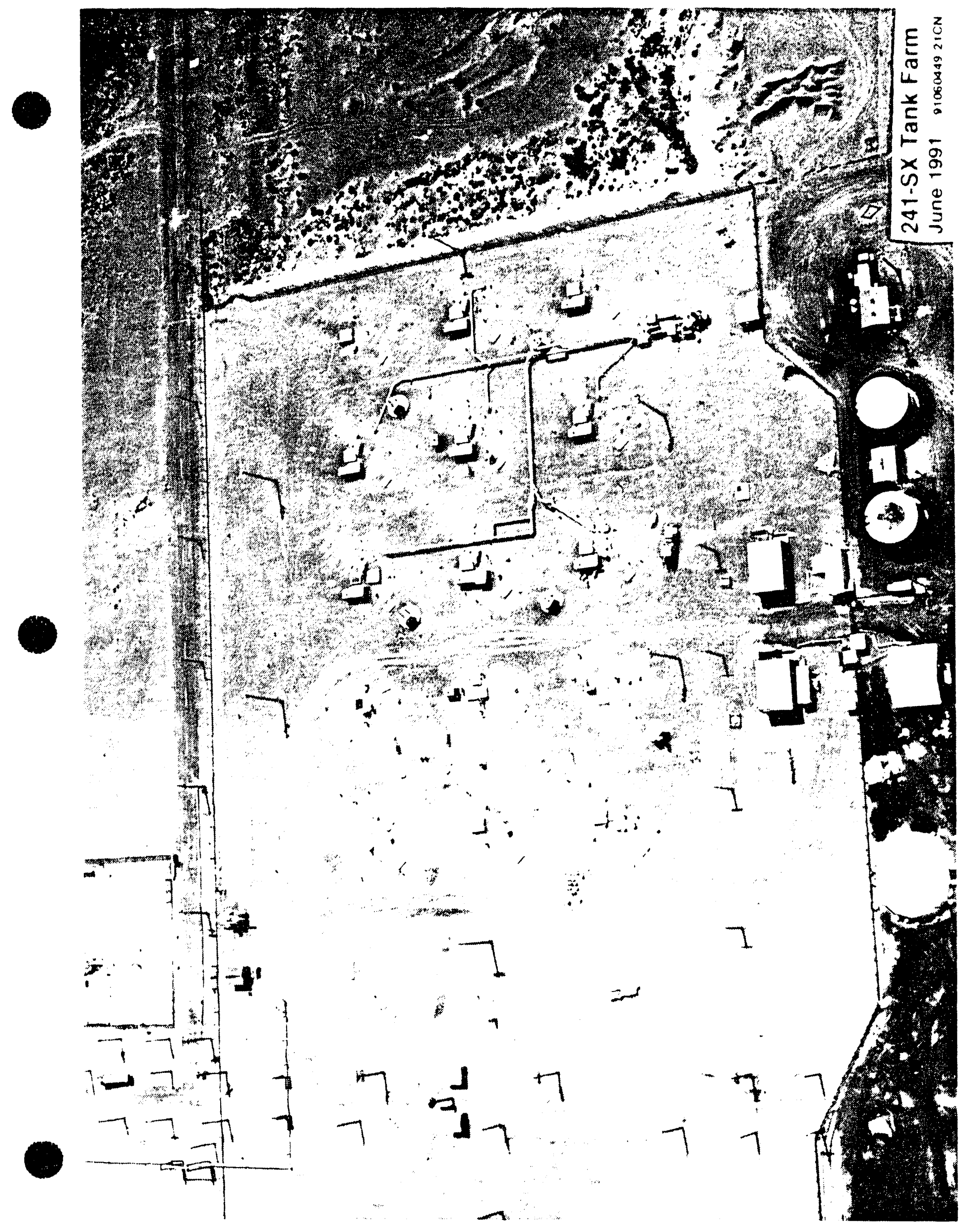




\section{WHC-EP-0625}

This page intentionally left blank. 


\section{Tank 241-SX-101}

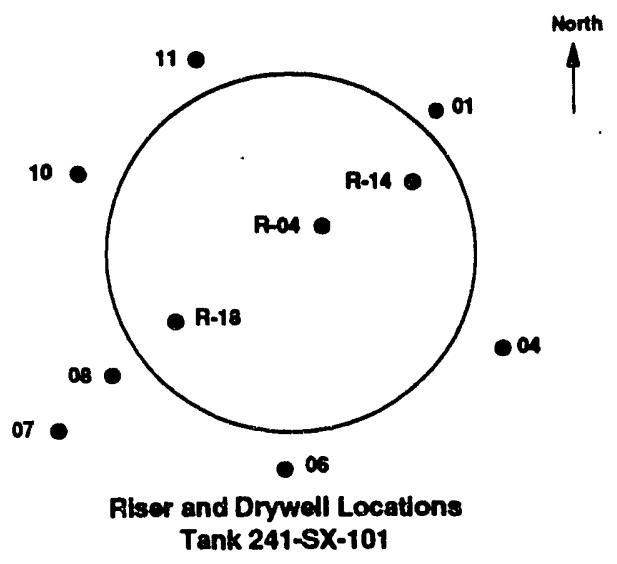

Number of External Drywells: 7

Number of Lateral Wells: None

\section{Tank Status}

Watch List: Hydrogen

Contents

Type: Dilute Complexed Waste

Total Waste: $456 \mathrm{~K}$ gallons

Supernate Volume: $1 \mathrm{~K}$ gallons

Drainable Interstitial Liquid: $145 \mathrm{~K}$ gallons

Isolation Status

Date Partially Interim Isolated: 06/85

Surface Level/Leak Status

Integrity Category: Sound

FIC Surface Level: Intrusion level set at 172.00 Inches $(12 / 28 / 92)$

Surface Level Comments: Intrusion Mode

Last Photographed: $03 / 10 / 89$

Photo Interpretation Comments: See attached photo.

Temperature Status

Highest temperature during 1992: $145.7^{\circ} \mathrm{F}(11 / 25 / 92)$

Comments: Temperatures are stable.

Drywell Status

Comments: Current drywell profiles were stable and consistent with established baseline profiles.
Tank Description

Type: Single Shell

Constructed: 1954

In-service: $05 / 54$

Out of Service: $11 / 21 / 80$

Diameter: $7^{\circ}$

Usable Depth: $30.9^{\prime}$

Capacity: 1,000K gallons

Dottom shape: Dish

Hanford Coordinates:

35,552' North

75,665' West

Ventllation: Operating Exhauster

\section{Leak Detection System}

Surface Level:

FIC Riser- R-04

Manual Tape Riser- R-18

LOW Riser(s)- R-14 
WHC-EP-0625

This page intentionally left blank. 


\section{Tank: 241-SX-101}

March 1999

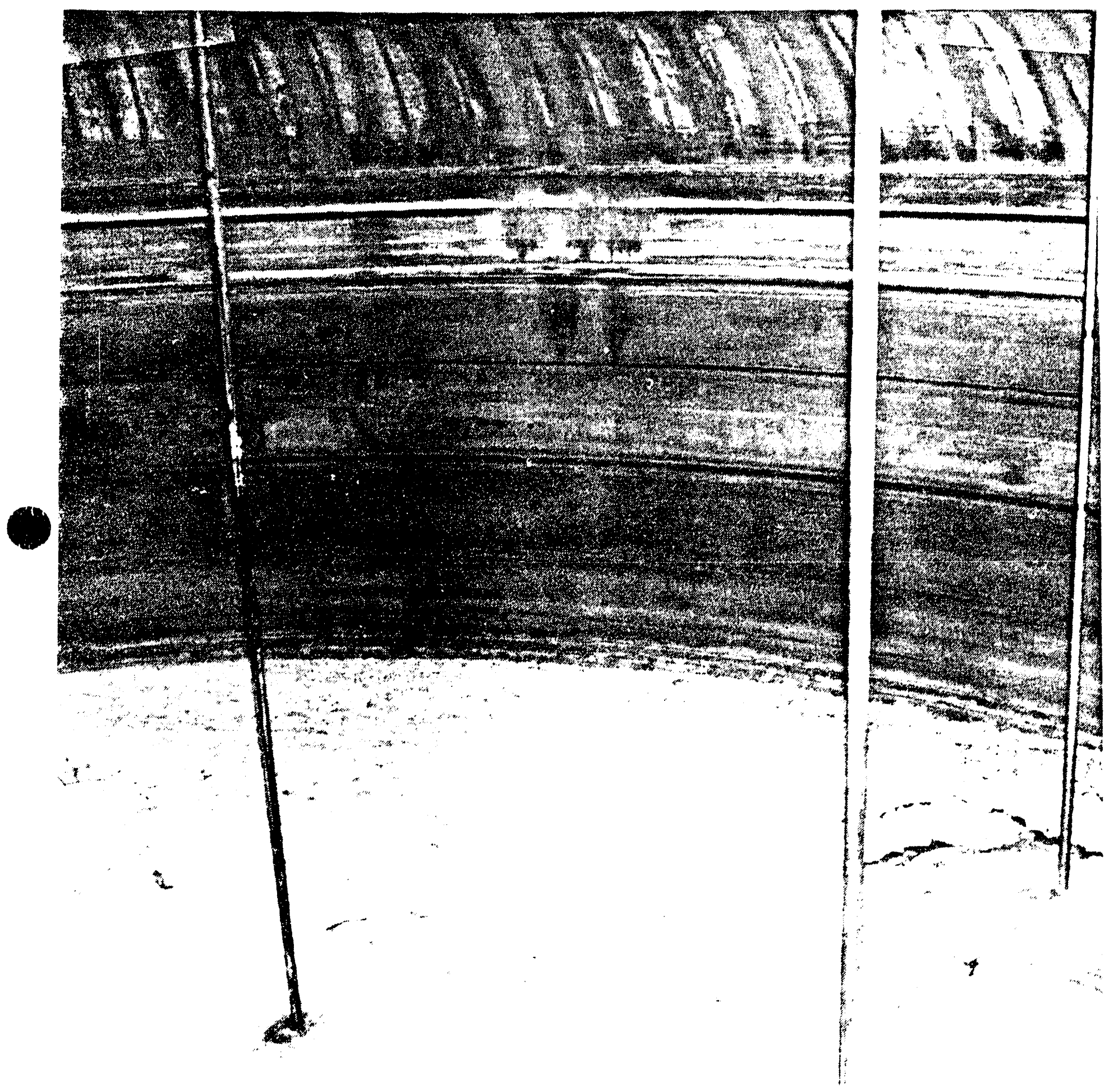


WHC-EP-0625

This page intentionally left blank. 


\section{Tank 241-SX-102}

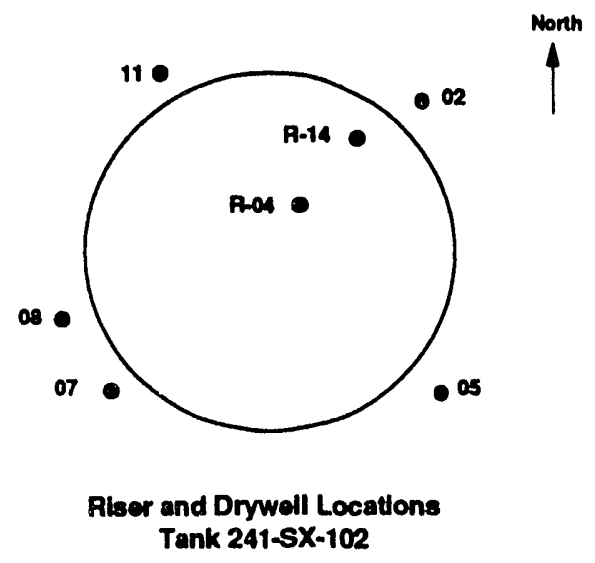

Number of External Drywells: 5

Number of Lateral Wells: None
Tank Description

Type: Single Shell

Constructed: 1954

In-service: $05 / 54$

Out of Service: $08 / 15 / 80$

Diameter: 75 '

Usable Depth: 30.9'

Capacity: $1,000 \mathrm{~K}$ gallons

Bottom shape: Dish

Hanford Coordinates:

35,552' North

75.767' West

Ventilation: Operating Exhauster

\section{Leak Detection System}

Surface Level:

FIC Riser- R-04

Manual Tape Riser- None

LOW Riser(s)- R-14

\section{Tank Status}

\section{Watch List: Hydrogen}

Contents

Type: Double Shell Slurry Feed

Total Waste: $543 \mathrm{~K}$ gallons

Supernate Volume: OK gallons

Drainable Interstitial Liquid: $183 \mathrm{~K}$ gallons

Isolation Status

Date Partially Interim Isolated: 06/85

\section{Surface Level/Leak Status}

Integrity Category: Sound

FIC Surface Level: Intrusion level set at 205.00 Inches (12/28/92)

Surface Level Comments: Intrusion Mode

Last Photographed: 01/07/88

Photo Interpretation Comments: See attached photo.

Temperature Status

Highest temperature during 1992: $157.6^{\circ} \mathrm{F}(12 / 27 / 92)$

Comments: Temperatures are stable.

\section{Drywell Status}

Comments: Current drywell profiles were stable and consistent with established baseline profiles. 


\section{WHC-EP-0625}

This page intentionally left blank. 


\section{rank $241.5 \times 102$}

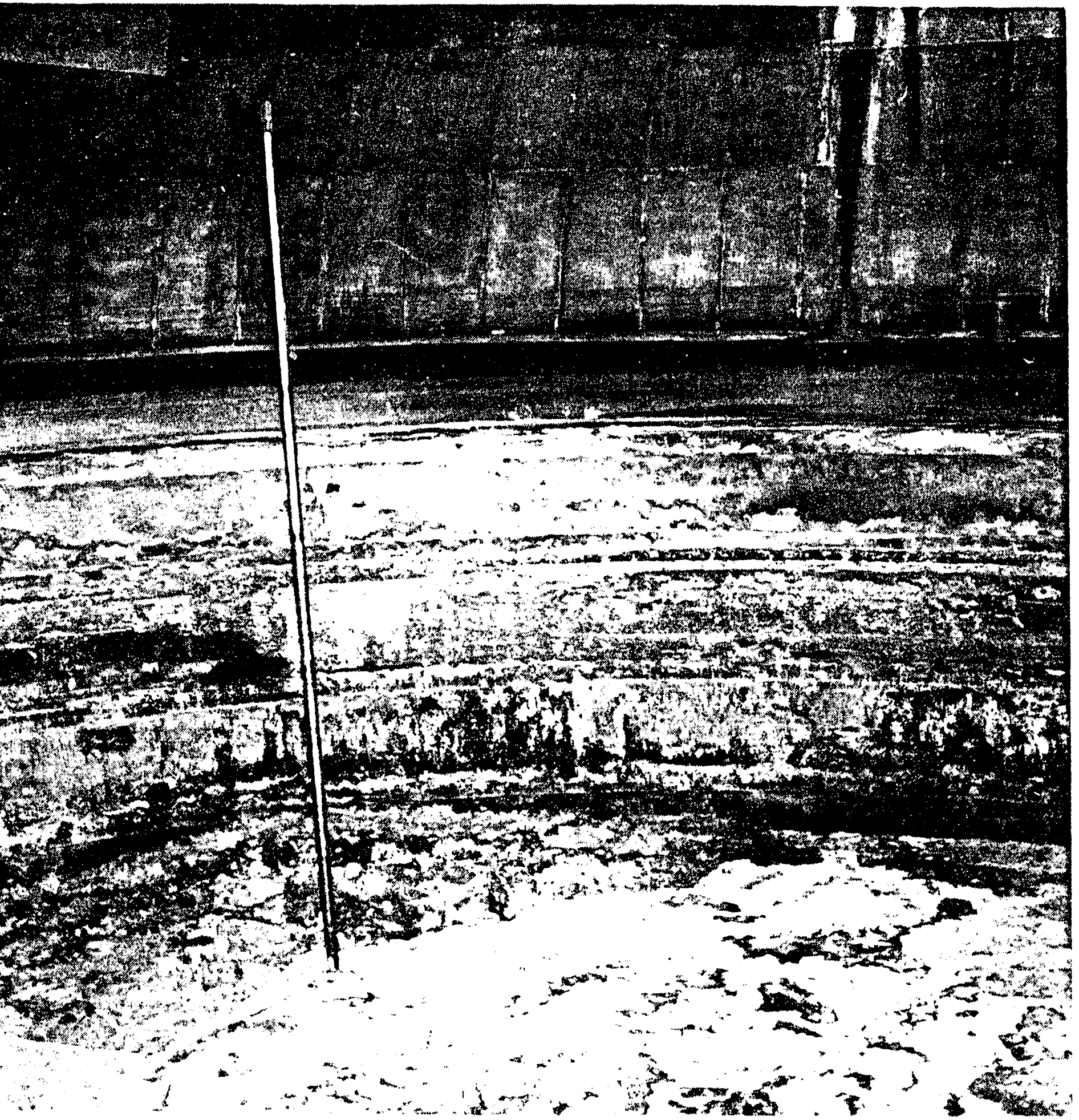


WHC-EP-0625

This page intentionally left blank. 


\section{Tank 241-SX-103}

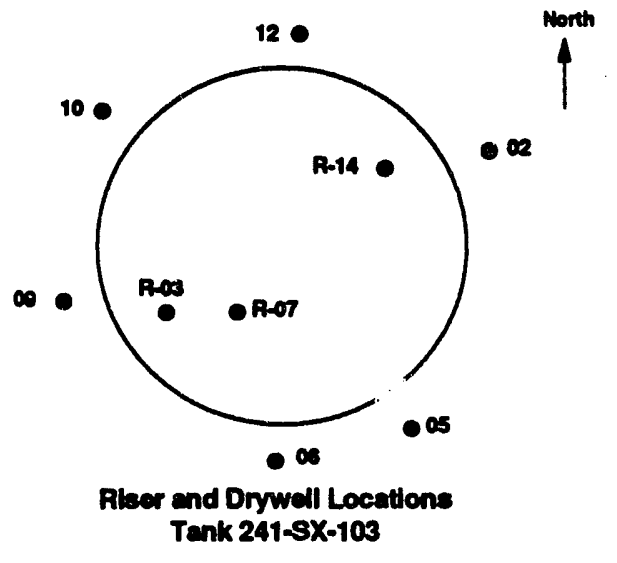

Number of External Drywelis: 6

Number of Lateral Wells: None

\section{Tank Status}

Watch List: Hydrogen

Contents

Type: Non-Complexed Waste

Total Waste: $652 \mathrm{~K}$ gallciss

Supernate Volume: $1 \mathrm{~K}$ gallons

Drainable Interstitial Liquid: $232 \mathrm{~K}$ gallons

Isolation Status

Date Partially Interim Isolated: 06/85

Surface Level/Leak Status

Integri, Category: Sound

FIC Surface Level: 242.50 Inches (12/10/92)

Last Photographed: 12/17/87

Photo Interpretation Comments: See attached photo.

Temperature Status

Highest temperature during 1992: $181.80{ }^{\circ} \mathrm{F}(01 / 10 / 92)$

Comments: Temperatures are stable.

Drywell Status

Cornments: Current drywell proflles were stable and consistent with established baseline profiles.
Tank Description

Type: Single Shell

Constructed: 1954

In-service: $05 / 54$

Out of Service: $08 / 27 / 80$

Diameter: $7^{\circ}$

Usable Depth: 30.9'

Cajuctu: 1,000K gallons

Bottom shine: Dish

Hanford Coordinates: 35.552' North 75,869' West

Ventilation: Operating Exhauster

\section{Leak Detection System}

Surface Level:

FIC Riser- R-03

Manual Tape: Riser- R-07

LOW Riser(s)- R-14 
WHC-EP-0625

This page intentionally left blank. 


\title{
Tank: 241-SX-103
}

\author{
December 1987
}

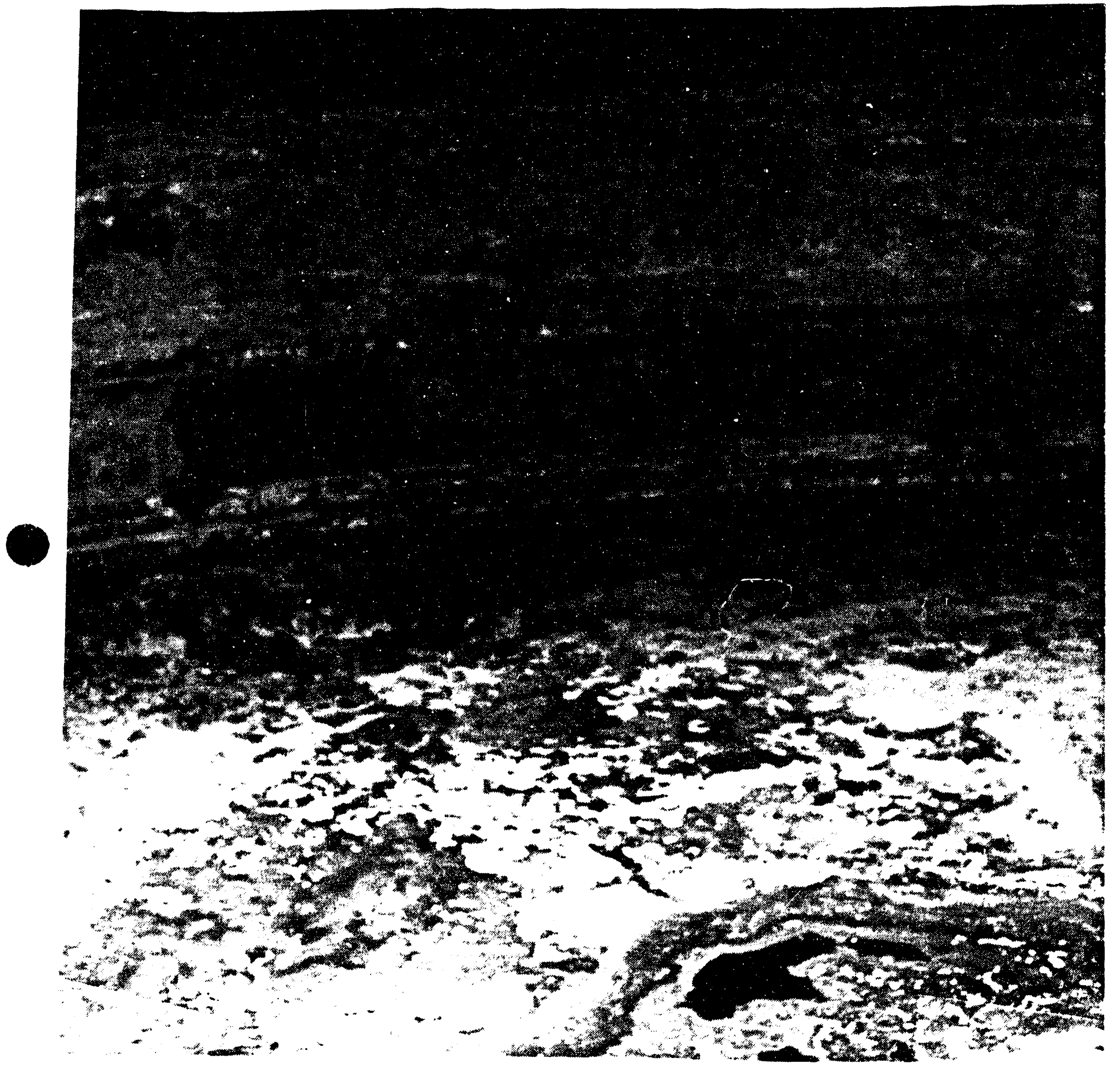

$8707708-22 \mathrm{CN}$ 
WHC-EP-0625

This page intentionally left blank. 


\section{Tank 241-SX-104}

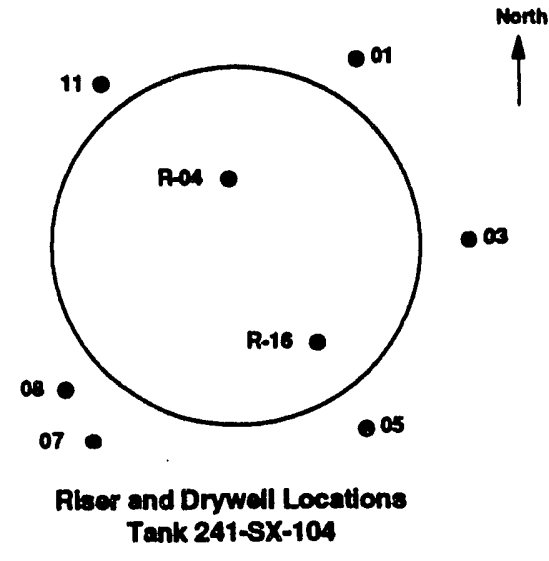

Number of External Drywells: 6

Number of Lateral Wells: None
Tank Description

Type: Single Shell

Constructed: 1954

In-service: $02 / 55$

Out of Service: $08 / 18 / 80$

Diameter: $75^{\prime}$

Usable Depth: 30.9'

Capacity: $1,000 \mathrm{~K}$ gallons

Bottom shape: Dish

Hanford Coordinates:

$35,450^{\prime}$ North

75,665' West

Ventilation: Operating Exhauster

\section{Leak Detection System}

Surface Level:

FIC Riser- R-04

Manual Tape Riser- None

LOW Riser(s)- R-16

\section{Tank Status}

Watch List: Hydrogen

Contents

Type: Double Shell Slurry Feed

Total Waste: $614 \mathrm{~K}$ gallons

Supernate Volume: OK gallons

Drainable Interstitial Liquid: $201 \mathrm{~K}$ gallons

Isolation Status

Date Partially Interim Isolated: 06/85

Surface Level/Leak Status

Integrity Category: Assumed Leaker

Date Declared Confirmed or Assumed Leaker: 1988

Leak Volume Estimate: 6,000 Gallons (1988)

FIC Surface Level: Intrusion level set at 237.00 Inches (12/28/92)

Surface Level Comments: Intrusion Mode

Last Photographed: 09/08/88

Photo Interpretation Comments: See attached photo.

Temperature Status

Highest temperature during 1992: $181^{\circ} \mathrm{F}(07 / 04 / 92)$

Comments: Temperatures are stable.

Drywell Status

Comments: Current drywell profiles were stable and consistent with established baseline profiles. 


\section{WHC-EP-0625}

This page intentionally left blank. 


\section{Tank: 241-SX-104}

September 1988
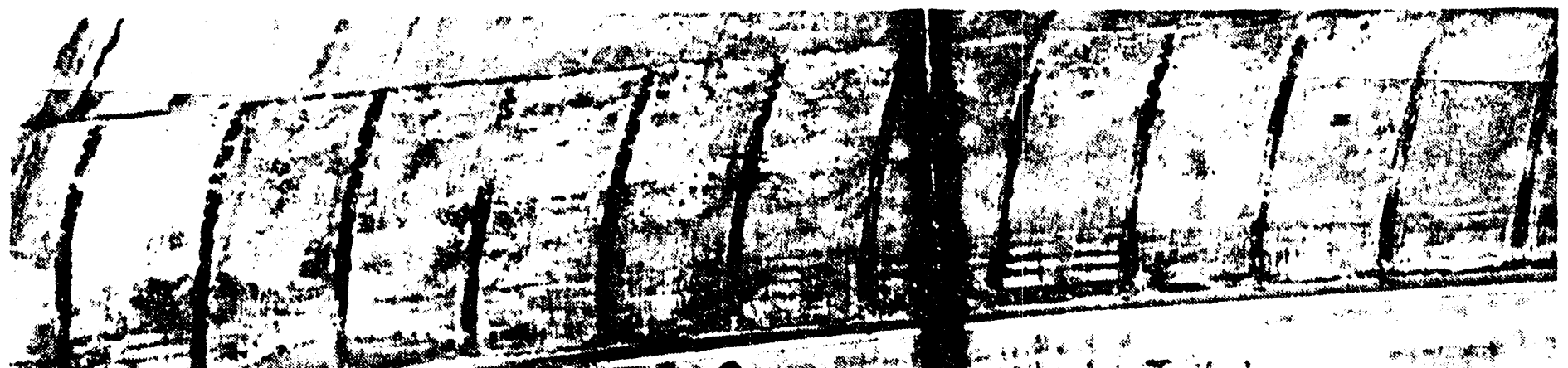

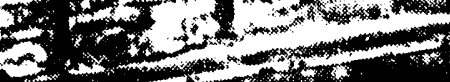

$+2$

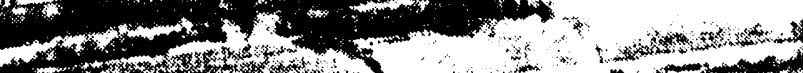
(2)

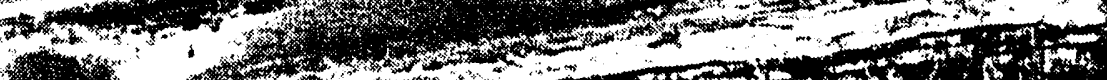
18 (3)

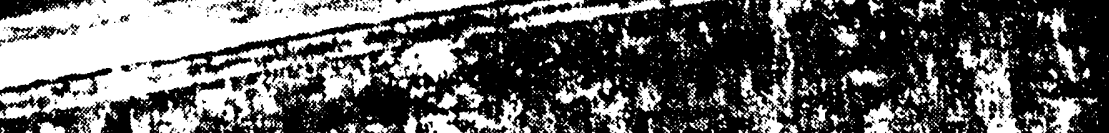

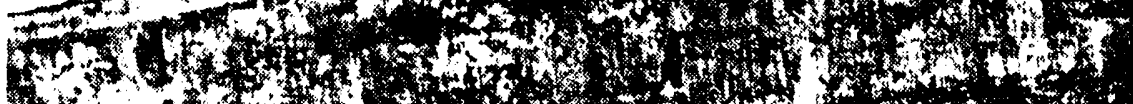

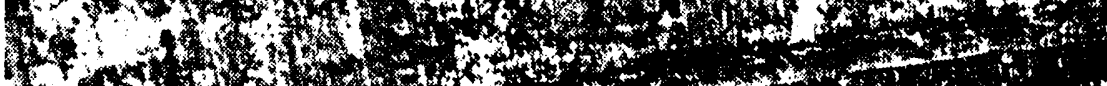

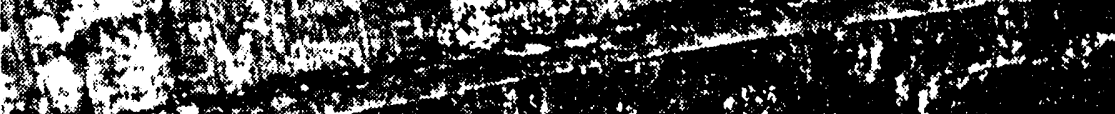

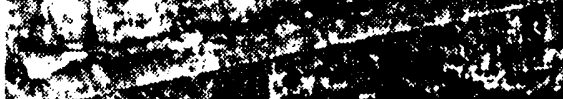

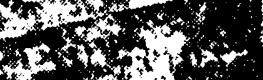

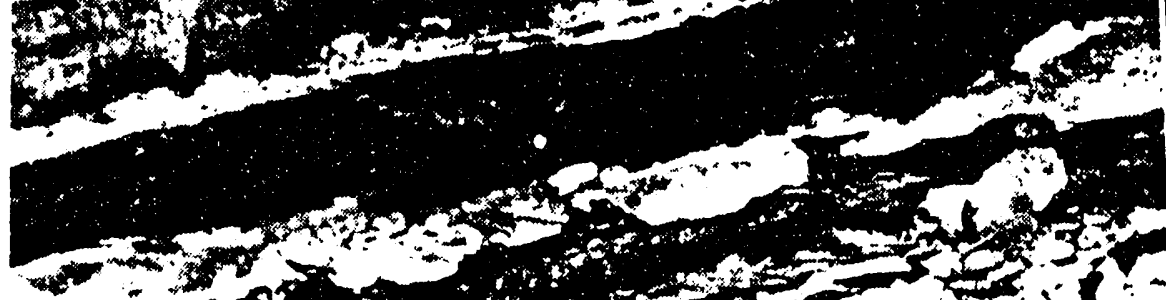
$\rightarrow+\infty$
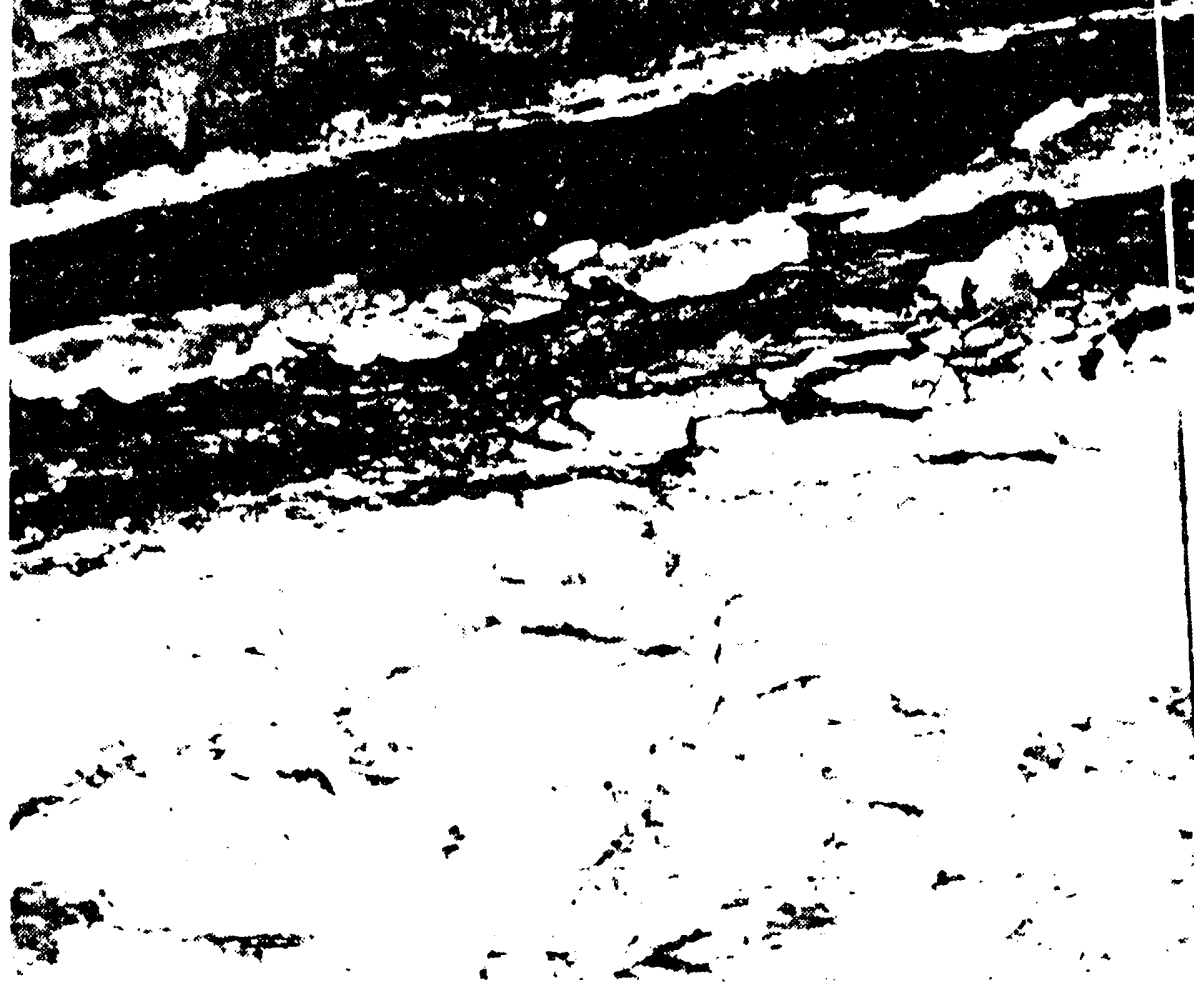

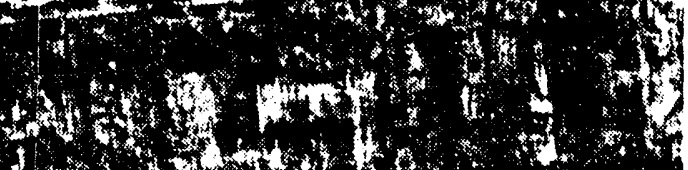

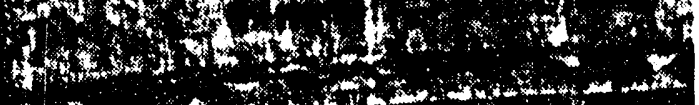

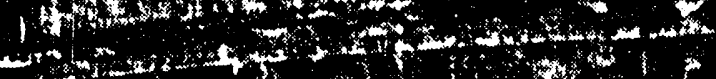
(3)

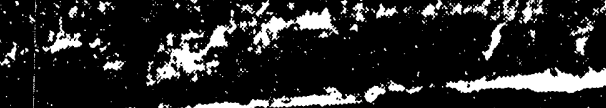

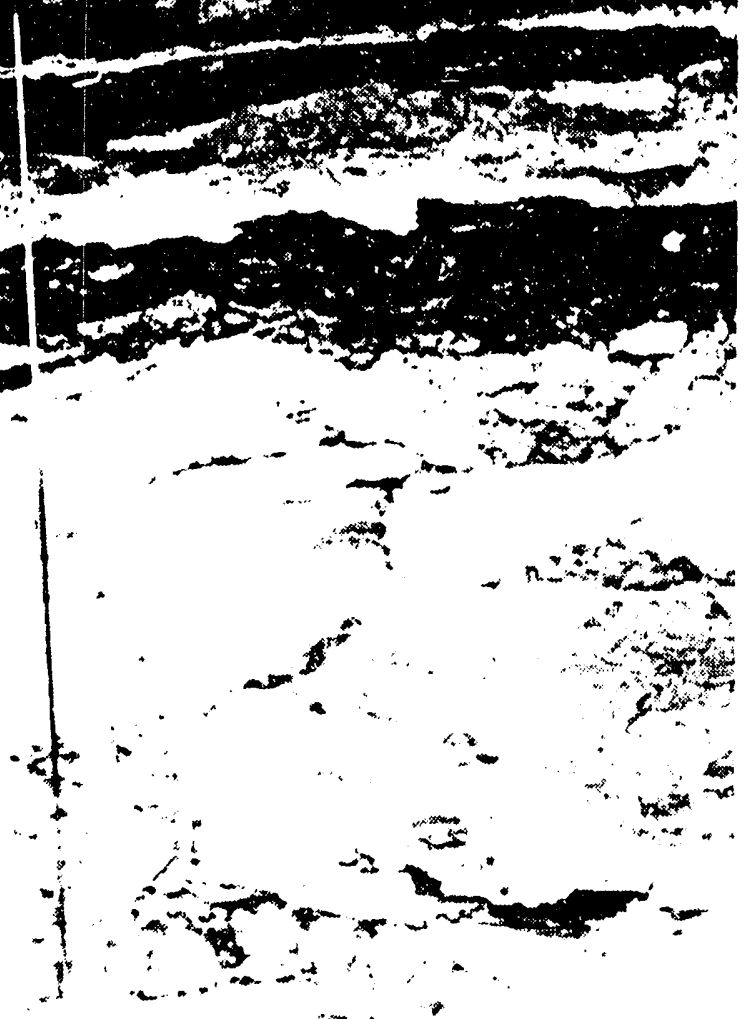


WHC-EP-0625

This page intentionally left blank. 


\section{Tank 241-SX-105}

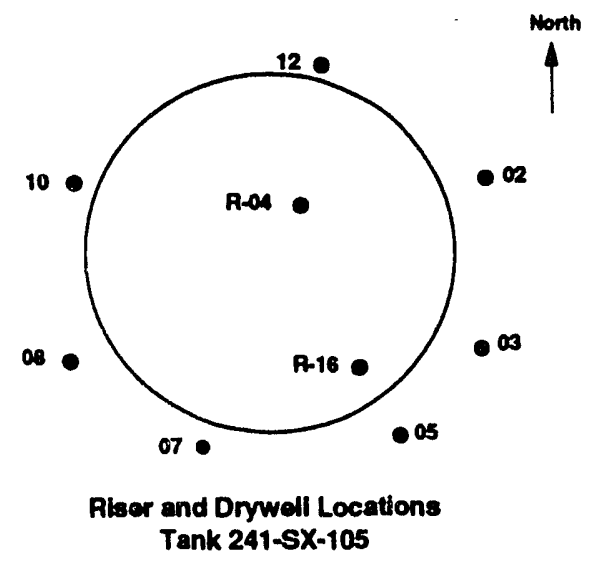

Number of External Drywells: 7

Number of Lateral Wells: 3

\section{Tank Status}

Watch List: Hydrogen

Contents

Type: Double Shell Slurry Feed

Total Waste: $683 \mathrm{~K}$ gallons

Supernate Volume: OK gallons

Drainable Interstitial Liquid: $261 \mathrm{~K}$ gallons

Isolation Status

Date Partially Interim Isolated: 06/85

\section{Surface Level/Leak Status}

Integrity Category: Sound

FIC Surface Level: Intrusion level set at 257.00 Inches (12/28/92)

Surface Level Comments: Intrusion Mode

Last Photographed: 06/15/88

Photo Interpretation Comments: See attached photo.

Temperature Status

Highest temperature during $1992: 189.3^{\circ} \mathrm{F}(01 / 10 / 92)$

Comments: Temperatures are stable.

Drywell Status

Comments: Current drywell profles were stable and consistent with established baseline profiles.
Tank Description

Type: Single Shell

Constructed: 1954

In-service: $02 / 55$

Out of Service: $08 / 29 / 80$

Diameter: $7^{\circ}$

Usable Depth: 30.9'

Capacity: $1,000 \mathrm{~K}$ gallons

Bottom shape: Dish

Hanford Coordinates: 35,450 ' North

75, 767' West

Ventilation: Operating Exhauster

\section{Leak Detection System}

Surface Level:

FIC Riser- R-04

Manual Tape Riser- None

LOW Riser(s)- R-16 


\section{WHC-EP-0625}

This page intentionally left blank. 


\section{Tank: 241-SX-105}

April 1983

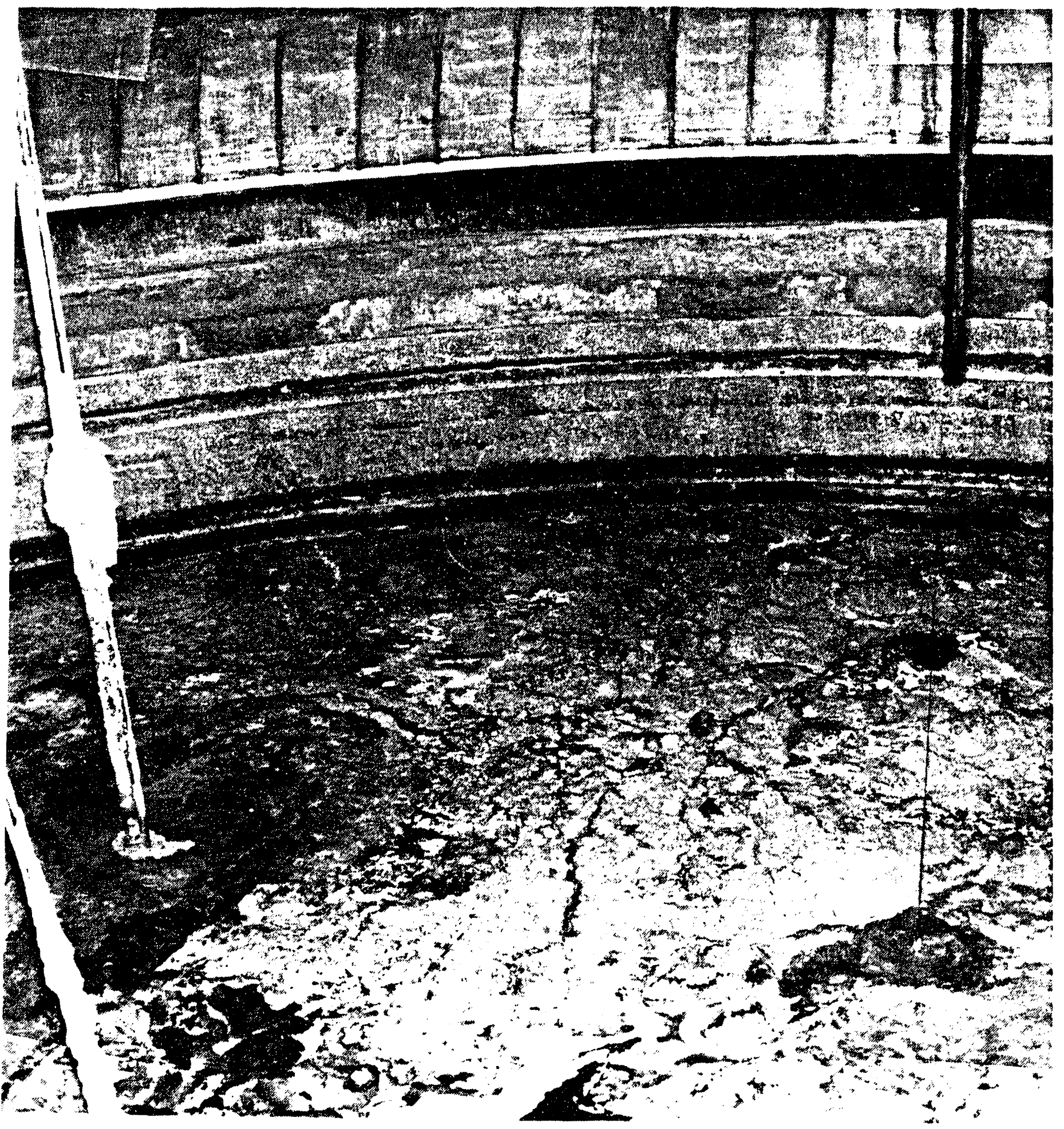




\section{WHC-EP-0625}

This page intentionally left blank. 


\section{Tank 241-SX-106}

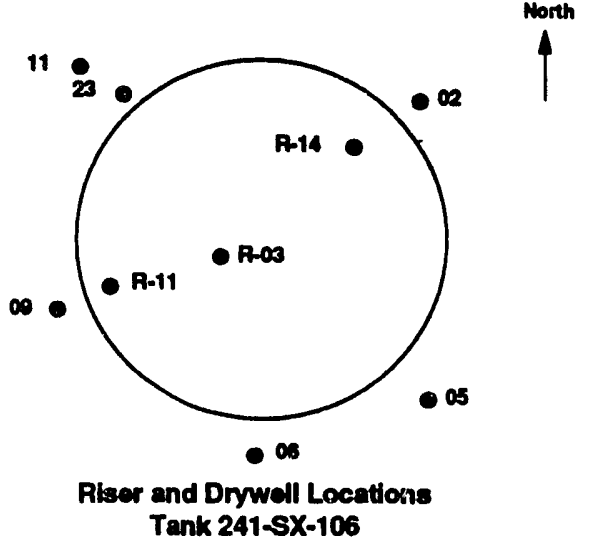

Number of External Drywells: 6 Number of Lateral Wells: None

\section{Tank Status}

Watch List: Hydrogen, Organic Salts

Contents

Type: Non-Complexed Waste

Total Waste: $538 \mathrm{~K}$ gallons

Supernate Volume: $61 \mathrm{~K}$ gallons

Drainable Interstitial Liquid: $194 \mathrm{~K}$ gallons

Isolation Status

Date Partially Interim Isolated: 06/85

\section{Surface Level/Leak Status}

Integrity Category: Sound

FIC Surface Level: 206.30 Inches (12/28/92)

Last Photographed: 06/01/89

Photo Interpretation Comments: See attached photo.

Temperature Status

Highest temperature during 1992: $116.5^{\circ} \mathrm{F}(03 / 01 / 92)$

Comments: Temperatures are stable.

Drywell Status

Comments: Current drywell profiles were stable and consistent with established baseline profiles.
Tank Description

Type: Single Shell

Constructed: 1954

In-service: 1954

Out of Service: $10 / 28 / 80$

Diameter: $75^{\prime}$

Usable Depth: 30.9'

Capacity: $1,000 \mathrm{~K}$ gallons

Bottom shape: Dish

Hanford Coordinates:

$35,450^{\prime}$ North

75,869 ' West

Venttlation: Operating Exhauster

\section{Leak Detection System}

Surface Level:

FIC Riser- R-03

Manual Tape Riser- R-1 1

LOW Riser(s)- R-14 
WHC-EP-0625

This page intentionally left blank. 
WHC HPH25

Tank: 241-SX-106

June 1989
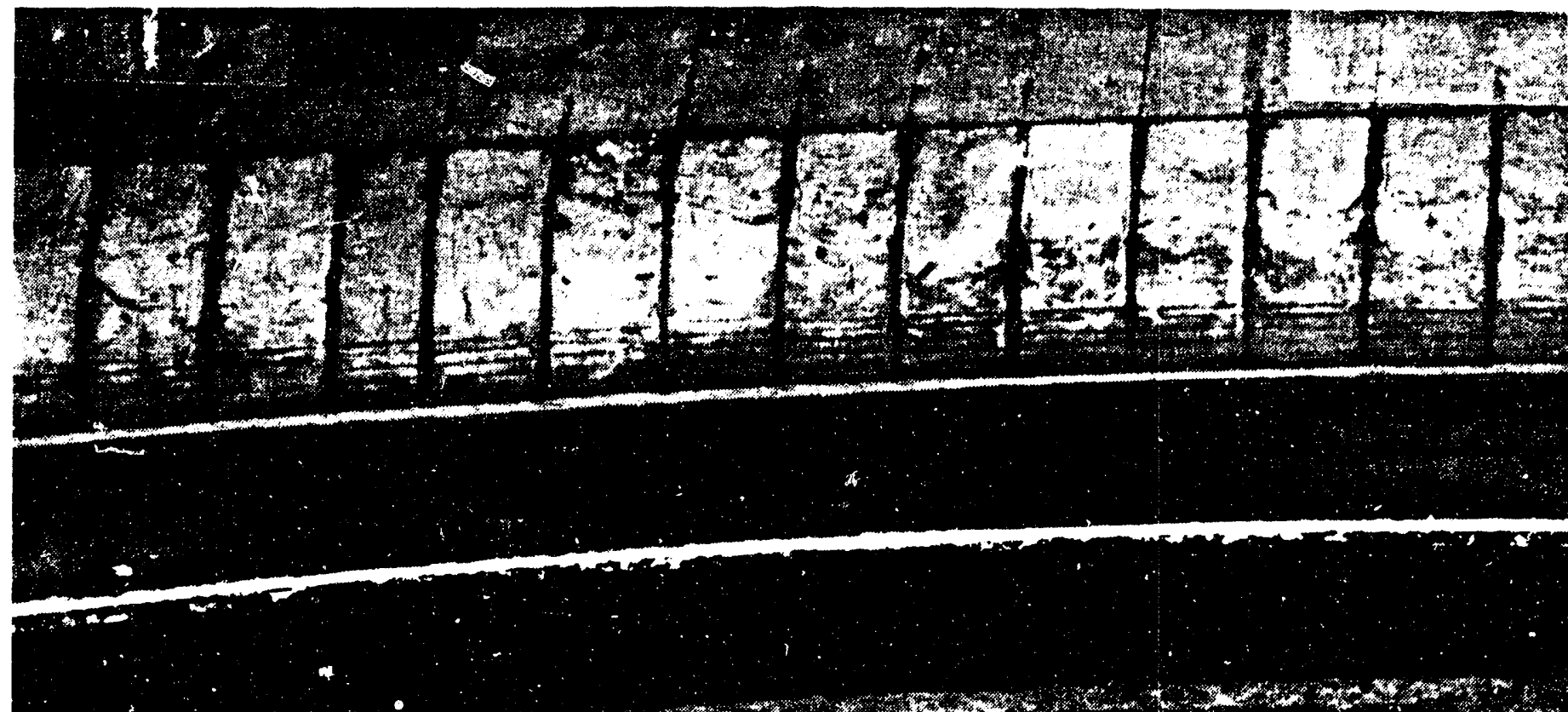

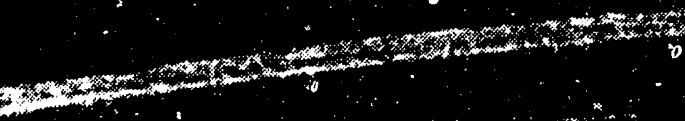

0

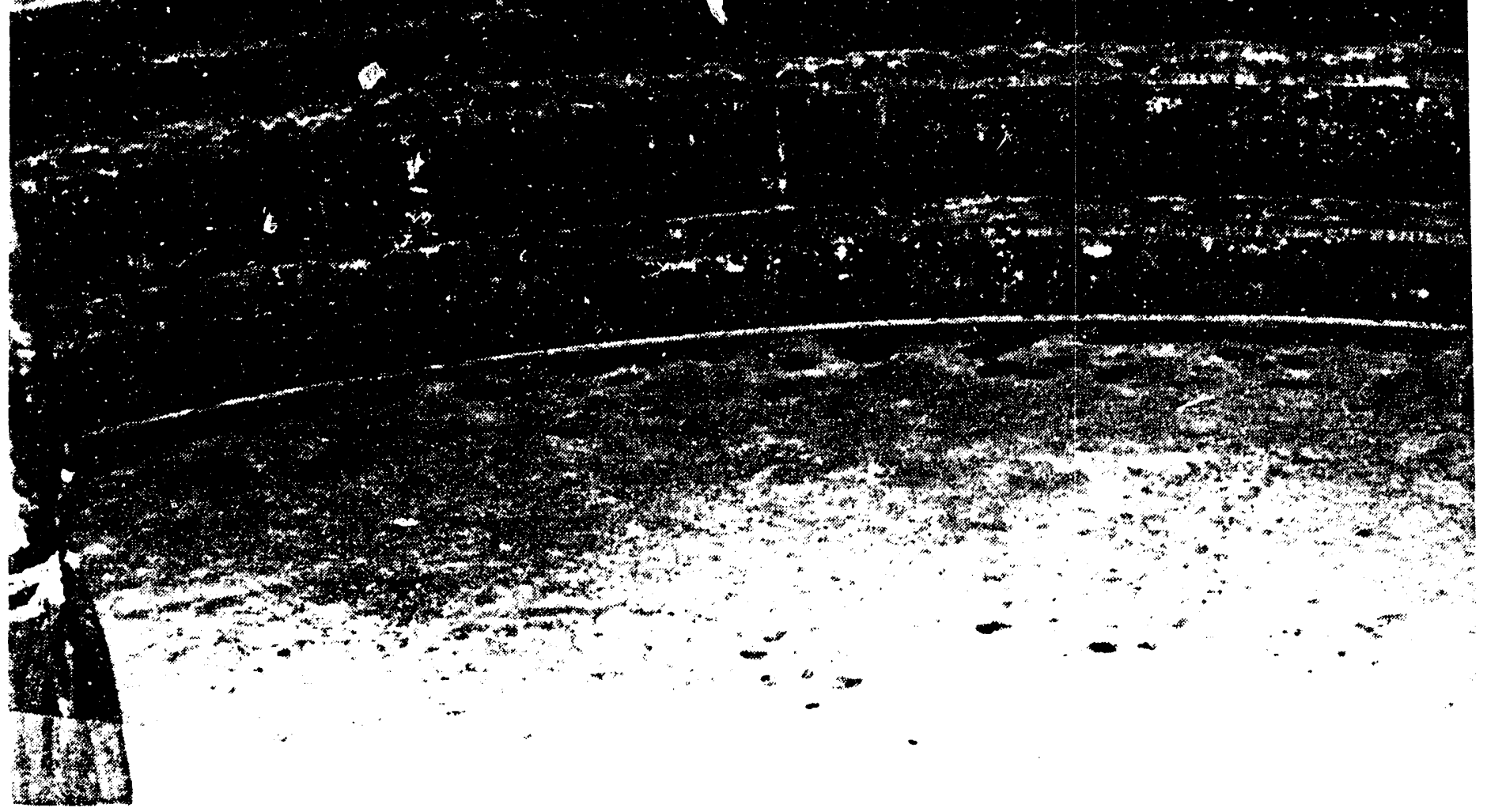


WHC-EP-0625

This page intentionally left blank. 


\section{Tank 241-SX-107}

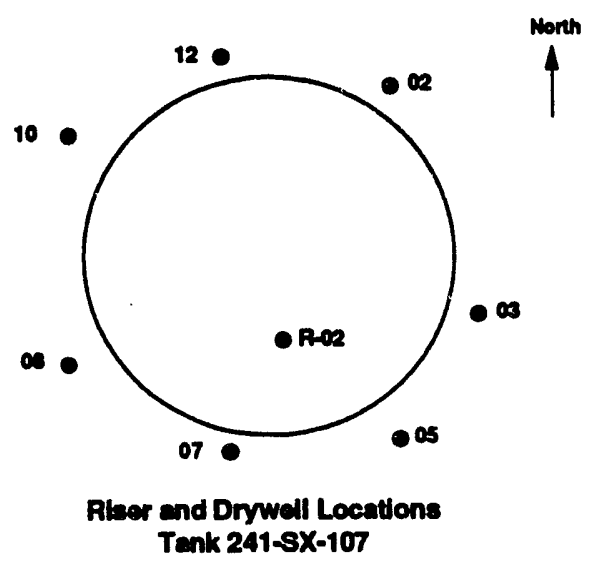

Number of External Drywells: 7

Number of Lateral Wells: 3

\section{Tank Status}

Contents

Type: Non-Complexed Waste

Total Waste: $104 \mathrm{~K}$ gallons

Supernate Volume: OK gallons

Drainable Interstitial Liquid: $5 \mathrm{~K}$ gallons

Isolation Status

Date Interim Stabilized: $10 / 19 / 79$

Date Interim Isolated: $09 / 82$

\section{Surface Level/Leak Status}

Integrity Category: Assumed Leaker

Date Declared Confirmed or Assumed Leaker: 1964

Leak Volume Estimate: < 5,000 Gallons (1983)

Manual Tape Surface Level: 40.50 Inches (10/15/92)

Last Photographed: 03/06/87

Photo Interpretation Comments:

Photographs show a dry, level caked surface with no visible liquid.

Temperature Status

Highest lemperature during 1992: $175^{\circ} \mathrm{F}(03 / 05 / 92)$

Comments: Temperatures are stable.

Drywell Status

Comments: Current drywell profiles were stable and consistent with established baseline profiles. 


\section{WHC-EP-0625}

This page intentionally left blank. 


\section{Tank 241-SX-108}

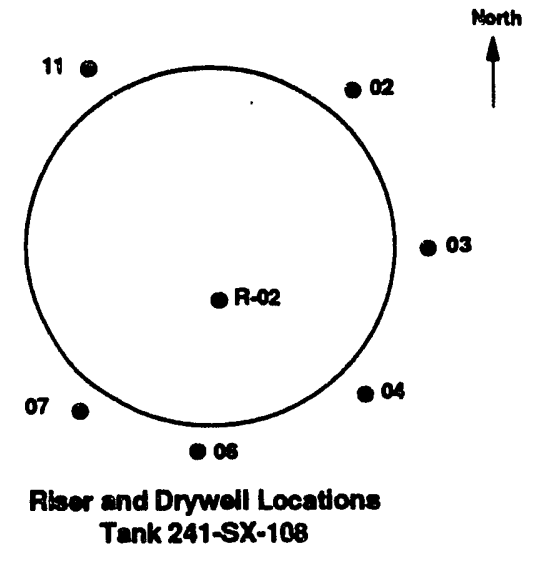

Number of External Drywells: 6 Number of Lateral Wells: 3

\section{Tank Status}

Contents

Type: Non-Complexed Waste

Total Waste: $115 \mathrm{~K}$ gallons

Supernate Volume: OK gallons

Drainable Interstitial Liquid: $6 \mathrm{~K}$ gallons

Isolation Status

Date Interim Stabilized: $08 / 79$

Date Interim Isolated: $12 / 15 / 82$

Surface Level/Leak Status

Integrity Category: Assumed Leaker

Date Declared Confirmed or Assumed Leaker: 1962

Leak Volume Estimate: 2,400 to 35,000 Gallons (1991)

Manual Tape Surface Level: 36.50 Inches (11/02/92)

Last Photographed: 03/06/87

Photo Interpretation Comments:

Photographs show a dry, caked surface and a discarded tape in the immediate area of the manual tape plummet.

\section{Temperature Status}

Highest temperature during 1992: $200.6^{\circ} \mathrm{F}(01 / 17 / 92)$

Comments: Temperatures are stable.

\section{Drywell Status}

Comments: Current drywell profiles were stable and consistent with established baseline profiles.
Tank Description Type: Single Shell Constructed: 1954 Cervice: $11 / 55$ Usable Depth: 30.9 Capacity: $1.000 \mathrm{~K}$ gallons Hanford Coordinates: 35,348 ' North $75,767^{\prime}$ ' West

Ventilation: Operating Exhauster

Surface Level:

FIC Riser- None

Manual Tape Riser- R-02

LOW Riser(s)- None shape: Dish

\section{Leak Detection System}


WHC-EP-0625

This page intentionally left blank. 


\section{Tank 241-SX-109}

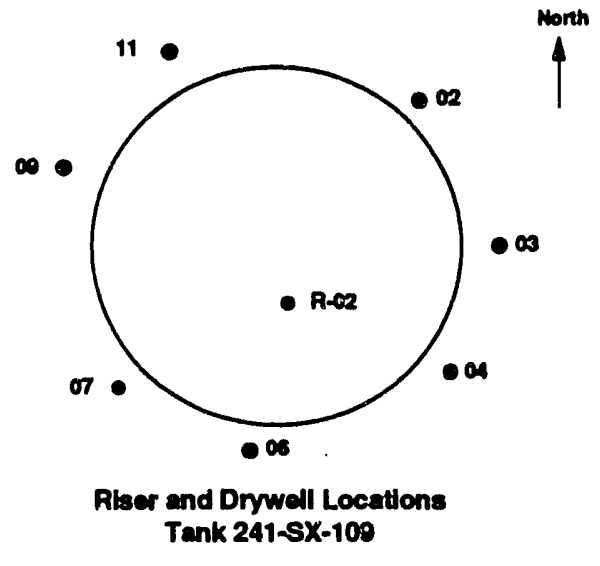

Number of External Drywells: 7
Tank Description

Type: Single Shell

Constructed: 1954

In-service: $09 / 55$

Out of Service: 1965

Diameter: $75^{\circ}$

Usable Depth: $30.9^{\prime}$

Capacity: $1,000 \mathrm{~K}$ gallons

Bottom shape: Dish

Hanford Coordinates:

$35,348^{\prime}$ North

75,869' West

Ventilation: Operating Exhauster

\section{Leak Detection System}

Surface Level:

FIC Riser- None

Manual Tape Riser- R-02

LOW Riser(s)- None

Number of Lateral Wells: 3

\section{Tank Status}

Watch List: Hydrogen potential, other tanks vent thru it

Contents

Type: Non-Complexed Waste

Total Waste: 250K gallons

Supernate Volume: OK gallons

Drainable Interstitial Liquid: $10 \mathrm{~K}$ gallons

Isolation Status

Date Interim Stabilized: $05 / 81$

Date Interim Isolated: $09 / 82$

Surface Level/Leak Status

Integrity Category: Assumed Leaker

Date Declared Confirmed or Assumed Leaker: 1965

Leak Volume Estimate: < 10,000 Gallons (1992)

Manual Tape Surface Level: 95.00 Inches (10/01/92)

Last Photographed: 05/21/86

Photo Interpretation Comments: See attached photo.

Temperature Status

Highest temperature during $1992: 162.20^{\circ} \mathrm{F}(01 / 17 / 92)$

Comments: Temperatures are stable.

Drywell Status

Comments: Current drywell profiles were stable and consistent with established baseline profules. 
WHC-EP-0625

This page intentionally left blank. 
WHC-EP-0625

\section{Tank: 241-SX-109}

\section{May 1986}
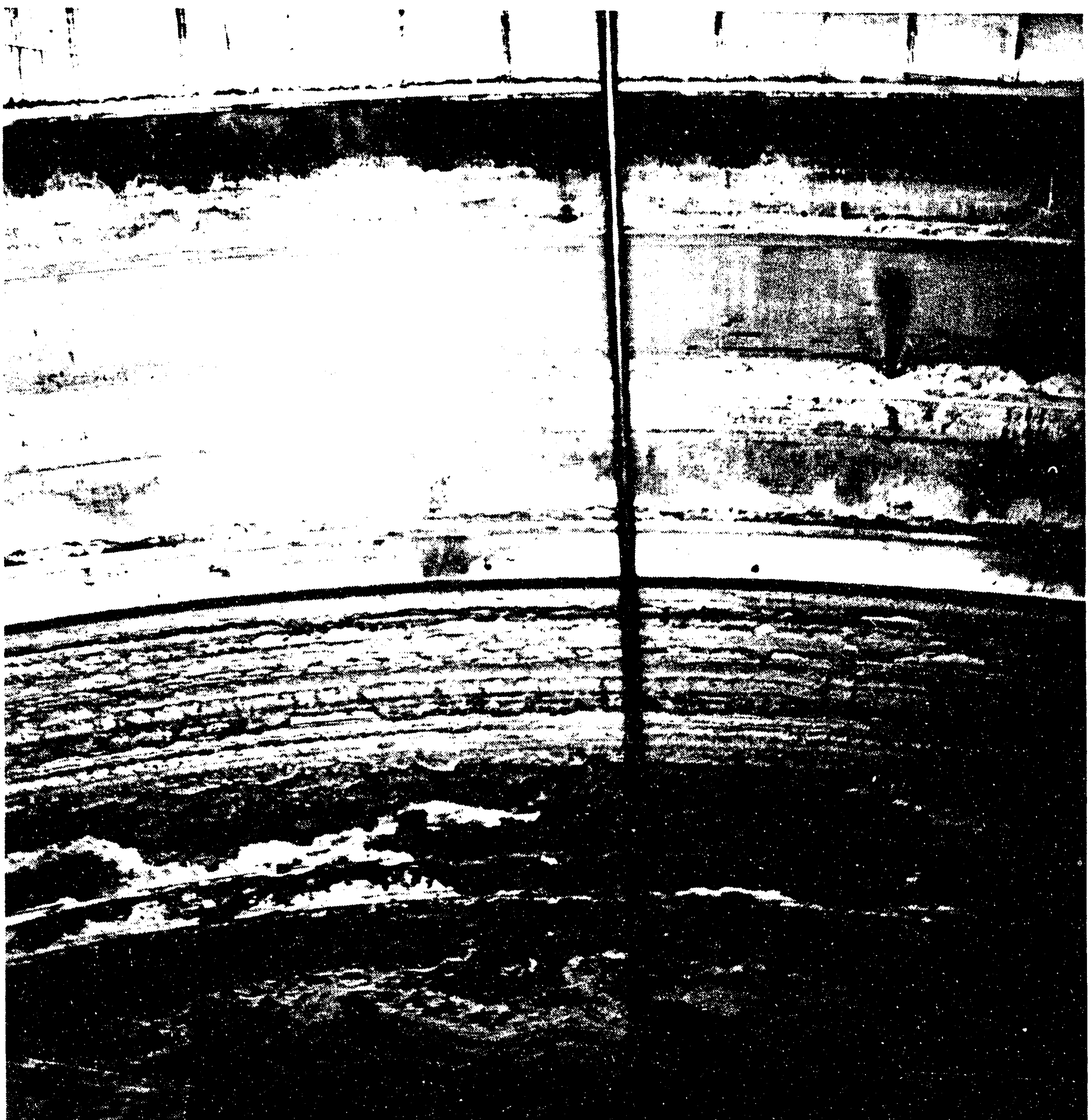

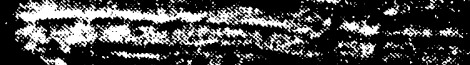

(2) $+2, \%$

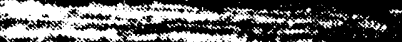

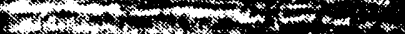

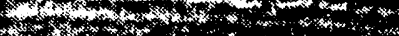

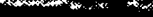


WHC-EP-0625

This page intentionally left blank. 


\section{Tank 241-SX-110}

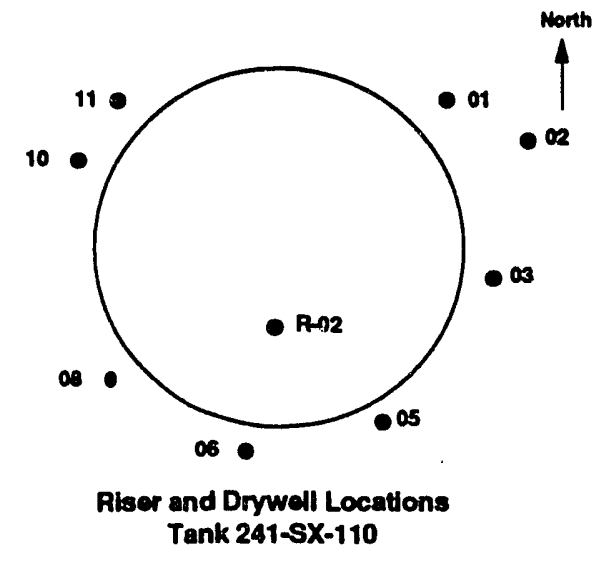

Number of External Drywells: 8

Number of Lateral Wells: 3

\section{Tank Status \\ Contents}

Type: Non-Complexed Waste

Total Waste: $62 \mathrm{~K}$ gallons

Supernate Volume: OK gallons

Drainable Interstitial Liquid: OK gallons

\section{Isolation Status}

Date Interim Stabilized: $08 / 79$

Date Interim Isolated: 06/14/85

\section{Surface Level/Leak Status}

Integrity Category: Assumed Leaker

Date Declared Confirmed or Assumed Leaker: 1976

Leak Volume Estimate: 5,500 Gallons (1989)

Manual Tape Surface Level: 26.75 inches (10/01/92)

Last Photographed: $02 / 20 / 87$

Photo Interpretation Comments:

Temperature Status

Photographs show a dry, caked surface with no visible liquid.

Highest temperature during 1992: $178^{\circ} \mathrm{F}(03 / 05 / 92)$

Comments: Temperatures are stable.

Drywell Status

Comments: Current drywell profiles were stable and consistent with established baseline profiles.
Tank Description

Type: Single Shell

Constructed: 1954

-service: $11 / 60$

Usable Depth: 30.9'

Capacity: $1,000 \mathrm{~K}$ gallons

Bottom shape: Dish

Hanford Coordinates:

$35,246^{\prime}$ North

75,665' West

Ventilation: Operating Exhauster

\section{Leak Detection System}

Surface Level:

FIC Riser- None

Manual Tape Riser- R-02

LOW Riser(s)- None 
WHC-EP-0625

This page intentionally left blank. 


\section{Tank 241-SX-111}

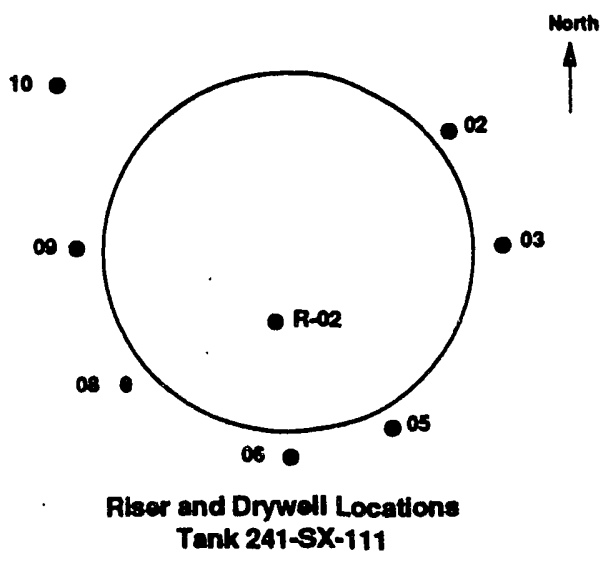

Tank Description

Type: Single Shell Constructed: 1954

In-service: $06 / 56$

Diameter: $7^{\circ}$

Usable Depth: $30.9^{\prime}$

Capacity: 1,000K gallons

Bottom shape: Dish

Hanford Coordinates:

35,246' North

$75,767^{\prime}$ West

Ventilation: Operating Exhauster

\section{Leak Detection System}

Surface Level:

FIC Riser- None

Manual Tape Riser- R-02

LOW Riser(s)- None

Number of External Drywells: 7

Number of Lateral Wells: 3

\section{Tank Status}

Contents

Type: Non-Complexed Waste

Total Waste: $125 \mathrm{~K}$ gallons

Supernate Volume: OK gallons

Drainable Interstitial Liquid: $7 \mathrm{~K}$ gallons

Isolation Status

Date Interim Stabilized: $07 / 79$

Date Intertm Isolated: $12 / 15 / 82$

\section{Surface Level/Leak Status}

Integrity Category: Assumed Leaker

Date Declared Confirmed or Assumed Leaker: 1974

Leak Volume Estimate: 2,000 Gallons (1986)

Manual Tape Surface Level: 49.75 Inches (10/01/92)

Last Photographed: 03/10/87

Photo Interpretation Comments:

Photographs show a dry, caked surface of sludge with no visible liquid.

Temperature Status

Highest temperature during $1992: 198.3^{\circ} \mathrm{F}(01 / 17 / 92)$

Comments: Temperatures are stable.

\section{Dryweil Status}

Comments: Current drywell profiles were stable and consistent with established baseline profiles. 
WHC-EP-0625

This page intentionally left blank. 


\section{Tank 241-SX-112}

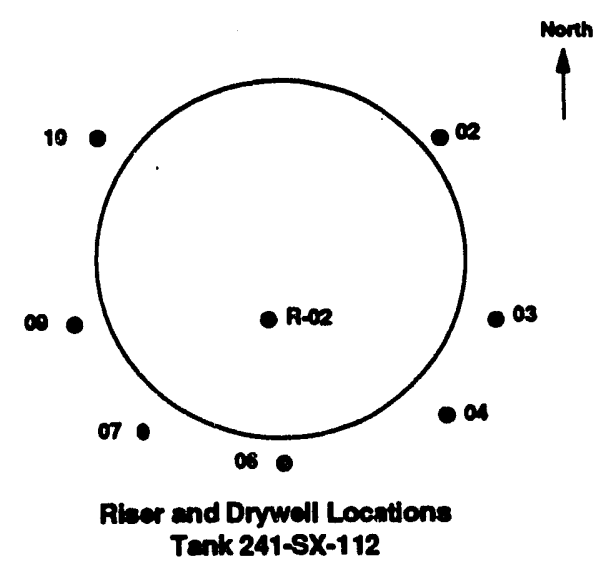

Number of External Drywells: 7

Number of Lateral Wells: 3

\section{Tank Status}

Contents

Type: Non-Complexed Waste

Total Waste: $92 \mathrm{~K}$ gallons

Supernate Volume: OK gallons

Dratnable Interstitial Liquid: $3 \mathrm{~K}$ gallons

isolation Status

Date Interim Stabilized: $07 / 79$

Date Interim Isolated: $12 / 15 / 82$

Surface Level/Leak Status

Integrity Category: Assumed Leaker

Date Declared Confirmed or Assumed Leaker: 1969

Leak Volume Estimate: 30,000 Gallons (1986)

Manual Tape Surface Level: 33.75 Inches (10/01/92)

Last Photographed: 03/10/87

Photo Interpretation Comments:

Photographs from 1974 show a liner crack as much as 3 in. wide (204-in. level). $4 / 18 / 84$ photographs reveal a sludge surface free of liquid. Discarded tapes and donoughnuts are in the immediate area of the manual tape plummet and measurement anomalies can be expected.

\section{Temperature Status}

Highest temperature during 1992: $1 € 2{ }^{\circ} \mathrm{F}(10 / 08 / 92)$

Comments: Temperatures are stable.

\section{Drywell Status}

Comments: Current drywell profiles were stable and consistent with established baseline profiles. 


\section{WHC-EP-0625}

This page intentionally left blank. 


\section{Tank 241-SX-113}

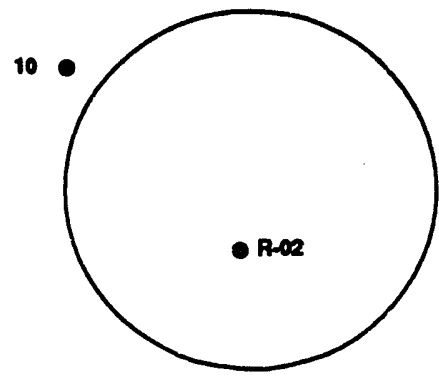

Rlser and Drywdl Loeations Tank 241-5x-113

Number of External Drywells: 1

Number of Lateral Wells: None

\section{Tank Status}

\section{Contents}

Type: Non-Complexed Waste

Total Waste: $26 \mathrm{~K}$ gallons

Supernate Volume: OK gallons

Drainable Interstitial Liquid: OK gallons

Isolation Status

Date Interim Stabilized: $11 / 30 / 78$

Date Interin Isolated: 09/82

\section{Surface Level/Leak Status}

Integrity Category: Assumed Leaker

Date Declared Confirmed or Assumed Leaker: 1962

Leak Volume Estimate: 15,000 Gallons (1986)

Manual Tape Surface Level: 15.00 Inches (10/15/92)

Last Photographed: 03/18/88

Photo Interpretation Comments:

Photographs show a dry, cracked surface in most areas but the surface appears

damp near the tank's center (as do previous photographs). The manual tape

Temperature Status plummet has a discarded manual tape directly beneath it.

Highest temperature during 1992: $80.4^{\circ} \mathrm{F}(01 / 01 / 92)$

Comments: Temperatures are stable.

Drywell Status

Comments: Current drywell proflles were stable and consistent with established baseline profiles. 


\section{WHC-EP-0625}

This page intentionally left blank. 


\section{Tank 241-SX-114}

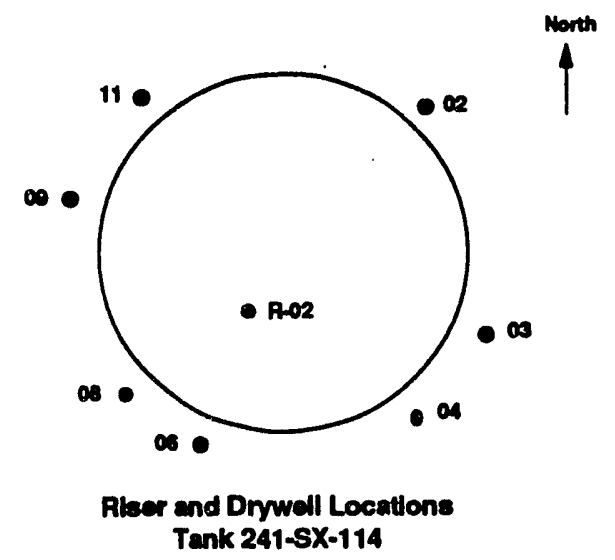

Number of External Drywells: 7

Number of Lateral Wells: 3

\section{Tank Status \\ Contents}

Type: Non-Complexed Waste

Total Waste: $181 \mathrm{~K}$ gallons

Supernate Volume: OK gallons

Drainable Interstitial Liquid: $14 \mathrm{~K}$ gallons

Isolation Status

Date Interim Stabilized: $07 / 79$

Date Interim Isolated: 09/82

\section{Surface Level/Leak Status}

Integrity Category: Assumed Leaker

Date Declared Confirmed or Assumed Leaker: 1972

Leak Volume Estimate: $7.8 \mathrm{~K}$ gallons $=1 / 19$ th of $150 \mathrm{~K}$ gallons

Manual Tape Surface Level: 66.00 Inches (10/01/92)

Last Photographed: 02/26/87

Photo Interpretation Comments:

Photographs show a dry surface of sludge with no visible liquid. The manual tape plummet appears to be in a small depression created by the plummet.

\section{Temperature Status}

Highest temperature during 1992: $192.5^{\circ} \mathrm{F}(08 / 07 / 92)$

Comments: Temperatures are stable.

\section{Drywell Status}

Comments: Current drywell profiles were stable and consistent with established baseline profiles. 


\section{WHC-EP-0625}

This page intentionally left blank. 


\section{Tank 241-SX-115}

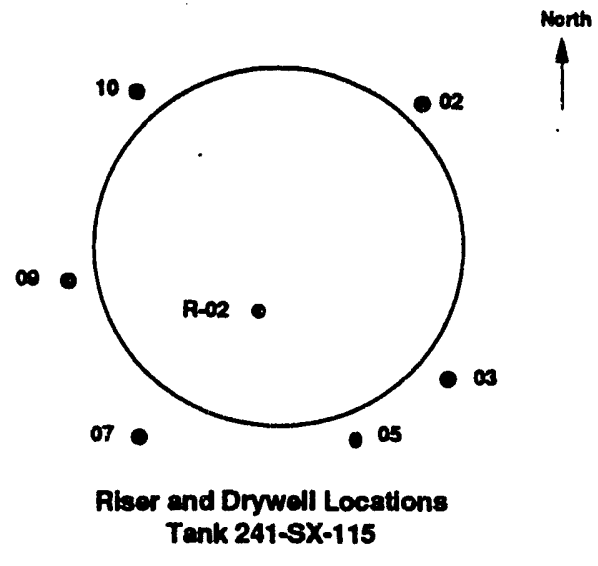

Number of External Drywells: 6 Number of Lateral Wells: 3

\section{Tank Status}

\section{Contents}

Type: Non-Complexed Waste

Total Waste: $12 \mathrm{~K}$ gallons

Supernate Volume: OK gallons

Drainable Interstitial Liquid: OK gallons

\section{Isolation Status}

Date Interim Stabilized: $09 / 30 / 78$

Date Interim Isolated: $12 . / 55 / 82$

\section{Surface Level/Leak Status}

Integrity Category: Assumed Leaker

Date Declared Confirmed or Assumed Leaker: 1965

Leak Volume Estimate: 50,000 Gallons (1986)

Manual Tape Surface Level: 7.00 Inches (10/01/92)

Last Photographed: 03/31/88

Photo Interpretation Comments:

\section{Temperature Status}

Photographs reveal a dry, level surface of sludge.

Highest temperature during 1992:

Drywell Status

Comments: Temperatures are stable.

Comments: Current drywell profiles were stable and consistent with established baseline profiles.
Tank Description

Type: Single Shell

Constructed: 1954

In-service: $09 / 58$

Out of Service: 1965

Diameter: 75 '

Usable Depth: $30.9^{\circ}$

Capacity: $1,000 \mathrm{~K}$ gallons

Bottom shape: Dish

Hanford Coordinates:

$35,144^{\prime}$ North

76,869' West

Ventllation: Passive

\section{Leak Detection System}

Surface Level:

FIC Riser- None

Manual Tape Riser- R-02

LOW Riser(s)- None 


\section{WHC-EP-0625}

This page intentionally left blank. 


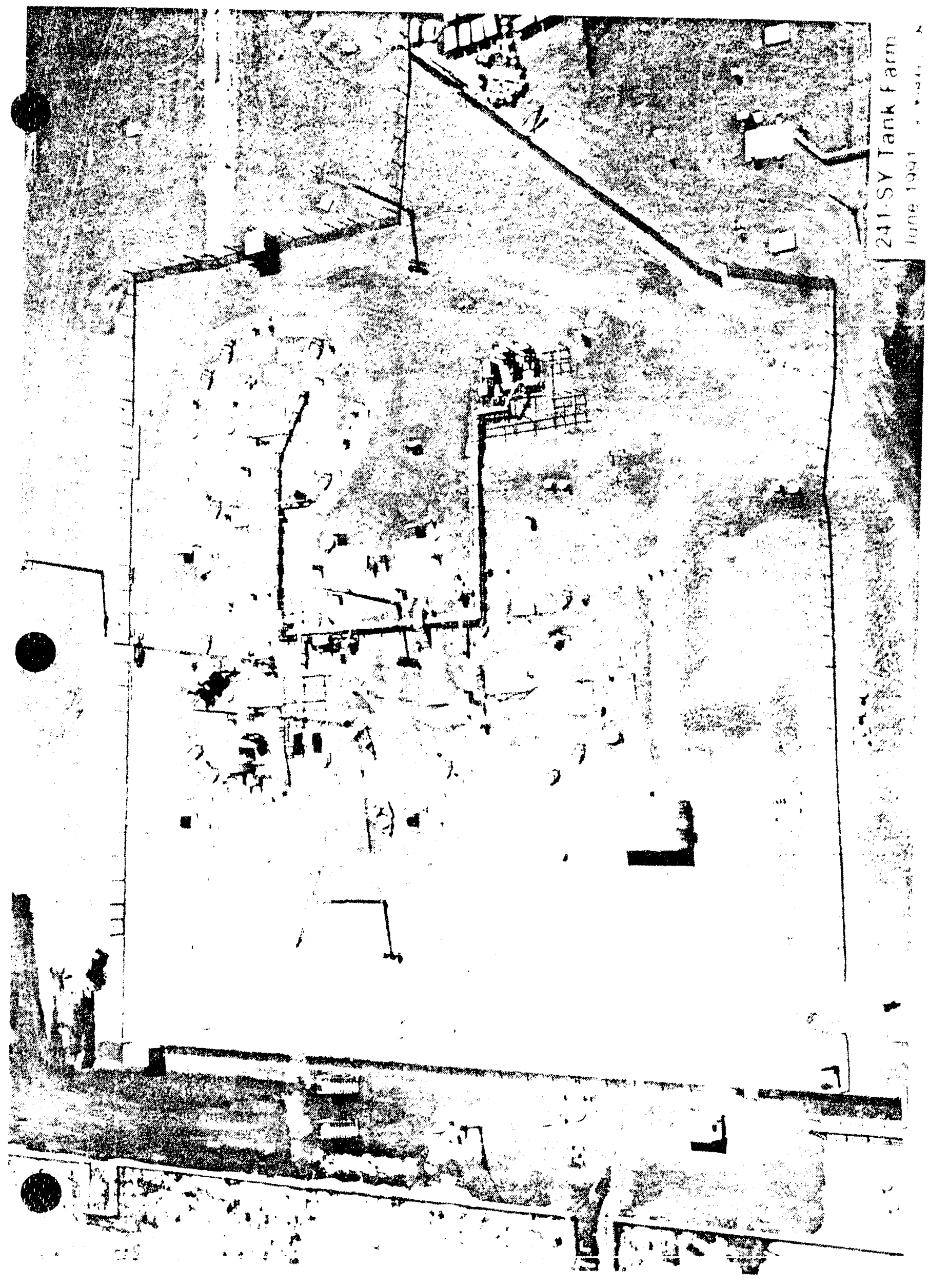




\section{WHC-EP-0625}

This page intentionally left blank. 


\section{Tank 241-SY-101}

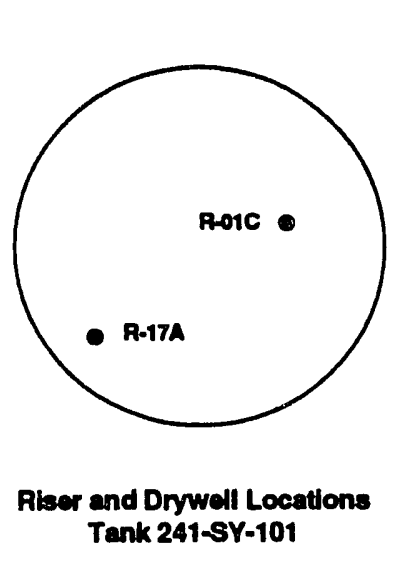

Number of External Drywells: None Number of Lateral Wells: None

\section{Tank Status}

Watch List: Hydrogen

Contents

Type: Complexant Concentrate Waste

Total Waste: $1127 \mathrm{~K}$ gallons

Supernate Volume: $37 \mathrm{~K}$ gallons

Drainable Interstitial Liquid: $237 \mathrm{~K}$ gallons

Surface Level/Leak Status

Integrity Category: Sound

Manual Tape Suface Level: 409.00 Inches (12/29/92)

FIC Surface Level: 408.70 Inches (12/29/92)

Last Photographed: 04/12/89

Photo Interpretation Comments: See attached photo.

Temperature Status

Highest temperature during 1992: $199.5^{\circ} \mathrm{F}(10 / 19 / 92)$

Comments: Temperatures are stable.
Tank Description

Type: Double Shell

Constructed: 1976

In-service: 1977

Diameter: $\mathbf{7 5}^{\circ}$

Usable Depth: 35.2'

Capacity: $1,160.5 \mathrm{~K}$ gallons

Bottom shape: Flat

Hanford Coordinates:

36.405' North

75,455' West

Ventilation: Operating Exhauster

\section{Leak Detection System}

Surface Level:

FIC Riser- R-01C

Manual Tape Riser- R-17A

LOW Riser(s)- None 
WHC-EP-0625

This page intentionally left blank. 


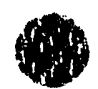


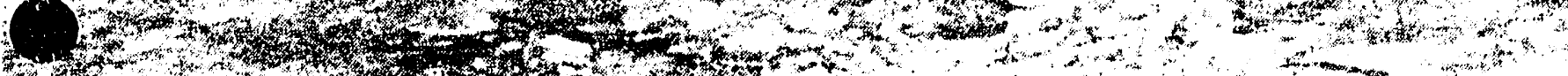

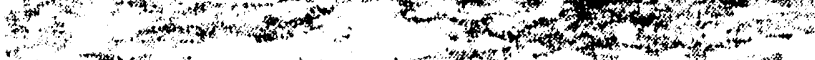
and

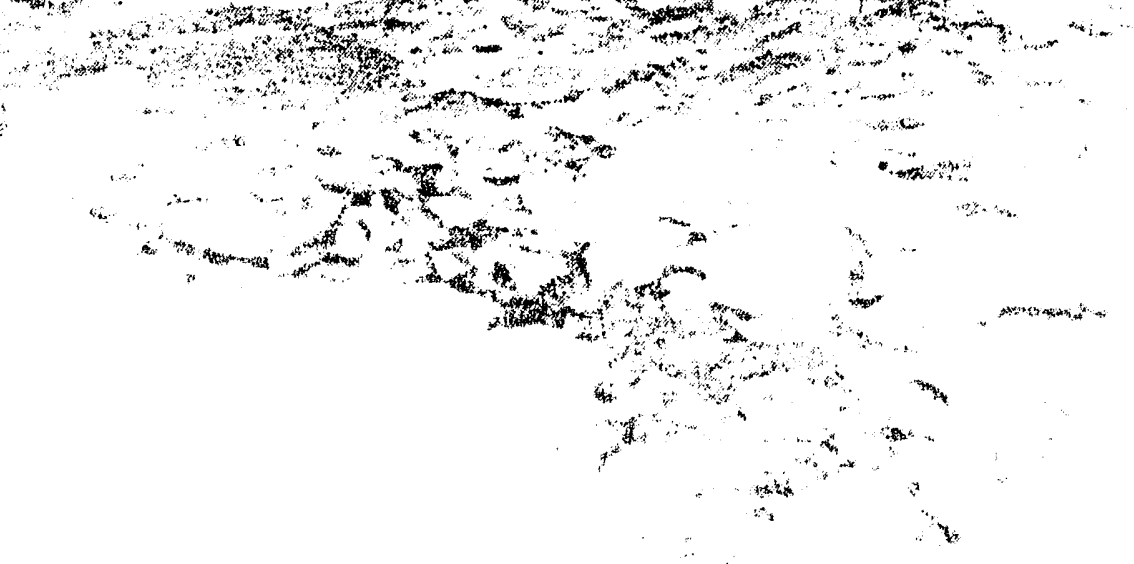


WHC-EP-0625

This page intentionally left blank. 


\section{Tank 241-SY-102}

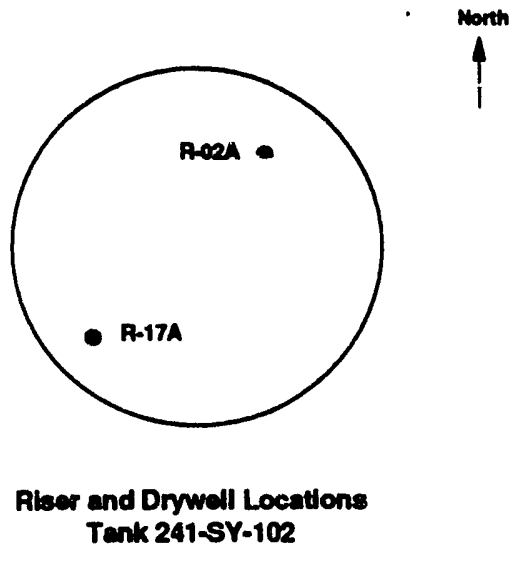

Number of External Drywells: None Number of Lateral Wells: None

\section{Tank Status}

Contents

Type: DN/PT

Total Waste: $675 \mathrm{~K}$ gallons

Supernate Volume: $604 \mathrm{~K}$ gallons

Drainahle Interstitial Liquid: OK gallons

Surface Level/Leak Status

Integrity Category: Sound

Manual Tape Surface Level: 245.00 Inches (12/29/92)

Last Photographed: 04/29/81

Photo Interpretation Comments:

No current photographs are avallable because tank 102-SY is active as a feed tank.

Temperature Status

Highest temperature during 1992: $73^{\circ} \mathrm{F}(09 / 07 / 92)$

Comments: Temperatures are stable.
Tank Description

Type: Double Shell

Constructed: 1976

In-service: 1977

Diameter: $\mathbf{7 5}^{\circ}$

Usable Depth: 35.2'

Capacity: $1,160.5 \mathrm{~K}$ gallons

Bottom shape: Flat

Hanford Coordinates:

$36,405^{\prime}$ North

75,562' West

Air Lift Circulators: Yes (1)

Ventilation: Operating Exhauster

\section{Leak Detection System}

Surface Level:

FIC Riser- R-02A

Manual Tape Riser- R-17A

LOW Riser(s)- None 


\section{WHC-EP-0625}

This page intentionally left blank. 


\section{Tank 241-SY-103}

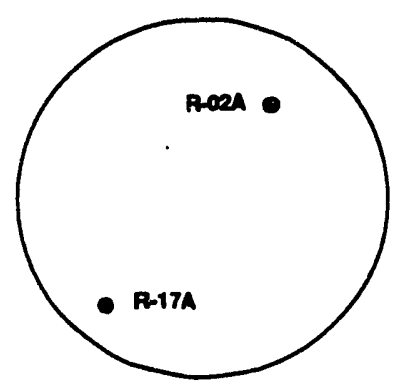

Riser and Drywell Locations Tank 241-SY-103

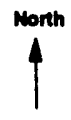

Number of External Drywells: None Number of Lateral Wells: None

\section{Tank Status}

\section{Watch List: Hydrogen}

Contents

Type: Complexant Concentrate Waste

Total Waste: $751 \mathrm{~K}$ gallons

Supernate Volume: $174 \mathrm{~K}$ gallons

Drainable Interstitial Liquid: OK gallons

Surface Level/Leak Status

Integrity Category: Sound

Manual Tape Surface Level: 274.00 Inches (12/29/92)

FIC Surface Level: 271.50 Inches (12/29/92)

Last Photographed: 10/01/85

Photo Interpretation Comments: See attached photo.

Temperature Status

Highest temperature during 1992: $143^{\circ} \mathrm{F}(01 / 27 / 92)$

Comments: Temperatures are stable.
Tank Description

Type: Double Shell

Constructed: 1976

In-service: 1977

Diameter: $\mathbf{7 5}^{\circ}$

Usable Depth: 35.2'

Capacity: $1,160.5 \mathrm{~K}$ gallons

Bottom shape: Flat

Hanford Coordinates:

36,298 ' North

75.562' West

Ventilation: Operating Exhauster

\section{Leak Detection System}

Surface Level:

FIC Riser- R-02A

Manual Tape Riser- R-17A

LOW Riser(s)- None 
WHC-EP-0625

This page intentionally left blank. 





WHC-EP-0625

This page intentionally left blank. 


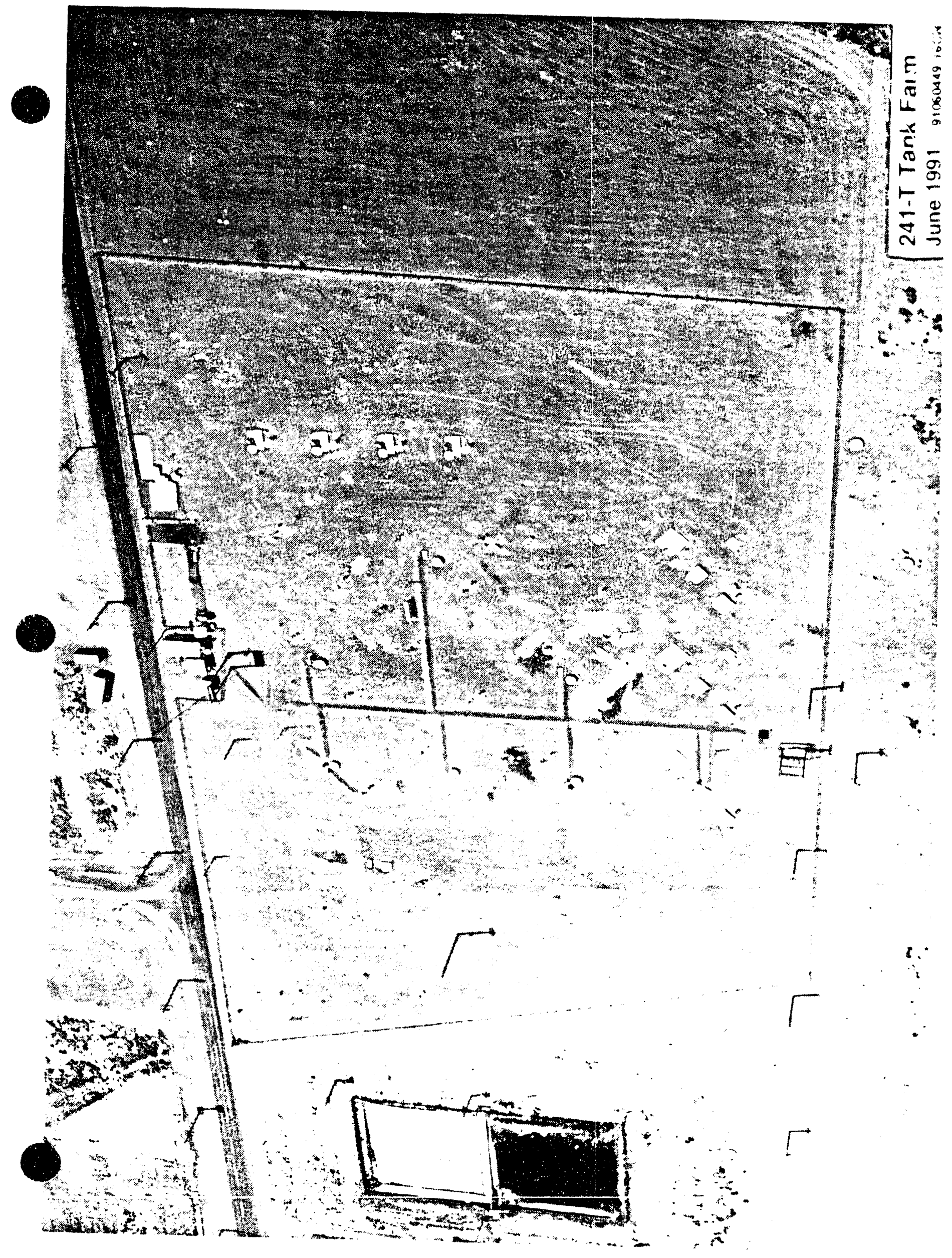


WHC-EP-0625

This page intentionally left blank. 


\section{Tank 241-T-101}

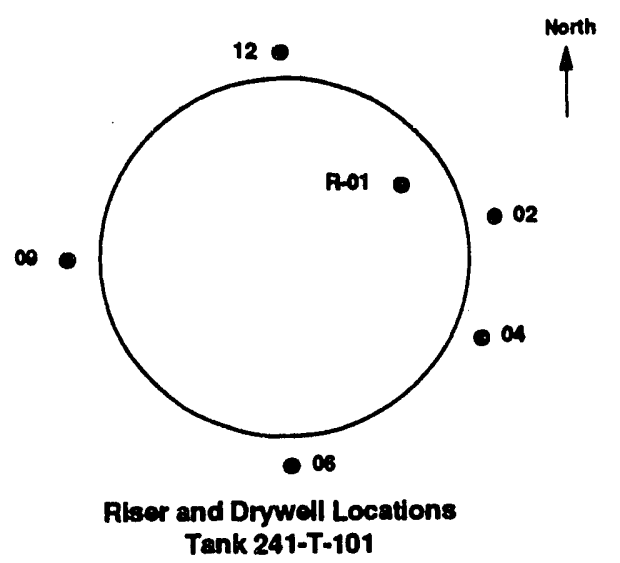

Number of External Drywells: 5

Number of Lateral Wells: None

\section{Tank Status}

Watch List: Ferrocyanide

Contents

Type: Non-Complexed Waste

Total Waste: 133K gallons

Supernate Volume $30 \mathrm{~K}$ gallons

Drainable Interstitial Liquid: $5 \mathrm{~K}$ gallons

Isolation Status

Date Partially Interim Isolated: $12 / 15 / 82$

Surface Level/Leak Status

Integrity Category: Sound

FIC Surface Level: 43.55 Inches $(01 / 01 / 93)$

Last Photographed: 07/03/84

Photo Interpretation Comments: See attached photo.

Temperature Status

Highest temperature during 1992: $76^{\circ} \mathrm{F}(11 / 19 / 92)$

Comments: Temperatures are stable.

\section{Drywell Status}

Comments: Current drywell profiles were stable and consistent with established baseline profiles.
Tank Description

Type: Single Shell

Constructed: 1944

In-service: $12 / 44$

Diameter: $7^{\prime}$

Usable Depth: 16'

Capacr.y: 530K gallons

Bottom shape: Dish

Hanford Coordinates: 43,64.7' North $75,637^{\circ}$ West

Ventilation: Passive

\section{Leak Detection System}

Surface Level:

FIC Riser- R-01

Manual Tape Riser- None

LOW Riser(s)- None 
WHC-EP-0625

This page intentionally left blank. 


\section{WHC-EP-0625}



\section{Tank: 241-T-101}

July 1984 
WHC-EP-0625

This page intentionally left blank. 


\section{Tank 241-T-102}

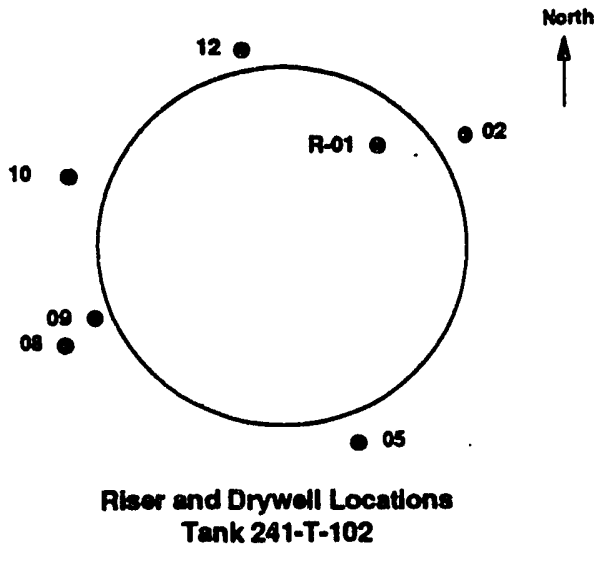

Number of External Drywells: 6
Tank Description

Type: Single Shell

Constructed: 1944

In-service: $09 / 45$

Out of Service: 1974

Dlameter: $75^{\prime}$

Usable Depth: 16'

Capacity: $530 \mathrm{~K}$ gallons

Bottom shape: Dish

Hanford Coordinates: $43,647^{\prime}$ North 75,737' West

Ventilation: Passive

\section{Leak Detection System}

Surface Level:

FIC Riser- R-01

Manual Tape Riser- None

LOW Riser(s)- None

Number of Lateral Wells: None

\section{Tank Status}

Contents

Type: Non-Complexed Waste

Total Waste: $32 \mathrm{~K}$ gallons

Supernate Volume: 13K gallons

Drainable Interstitial Liquid: OK gallons

Isolation Status

Date Interim Stabilized: $03 / 81$

Date Interim Isolated: $08 / 81$

\section{Surface Level/Leak Status}

Integrity Category: Sound

FIC Surface Level: 7.10 Inches $(12 / 21 / 92)$

Last Photographed: 06/28/89

Photo Interpretation Comments:

Photographs from $08 / 01 / 83$ and $11 / 08 / 83$ continue to show more surface liquid as do photographs back to 1978 . The surface is now shallow liquid except for a band of exposed sludge around the tank perimeter. The FIC plummet is contacting liquid $(8 / 1 / 83$ photographs).

Temperature Status

Highest temperature during 1992:

Comments: Temperatures are stabie.

\section{Drywell Status}

Comments: Current drywell proflles were stable and consistent with established baseline profiles. 
WHC-EP-0625

This page intentionally left blank. 


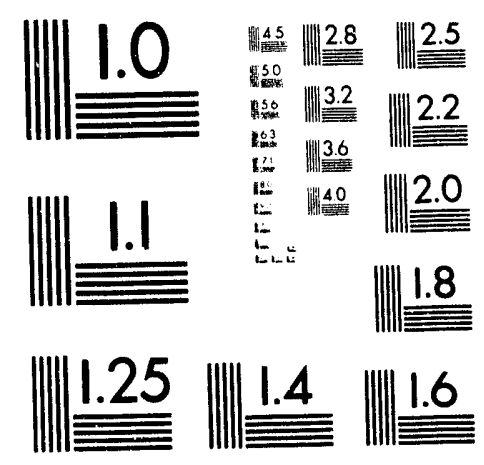



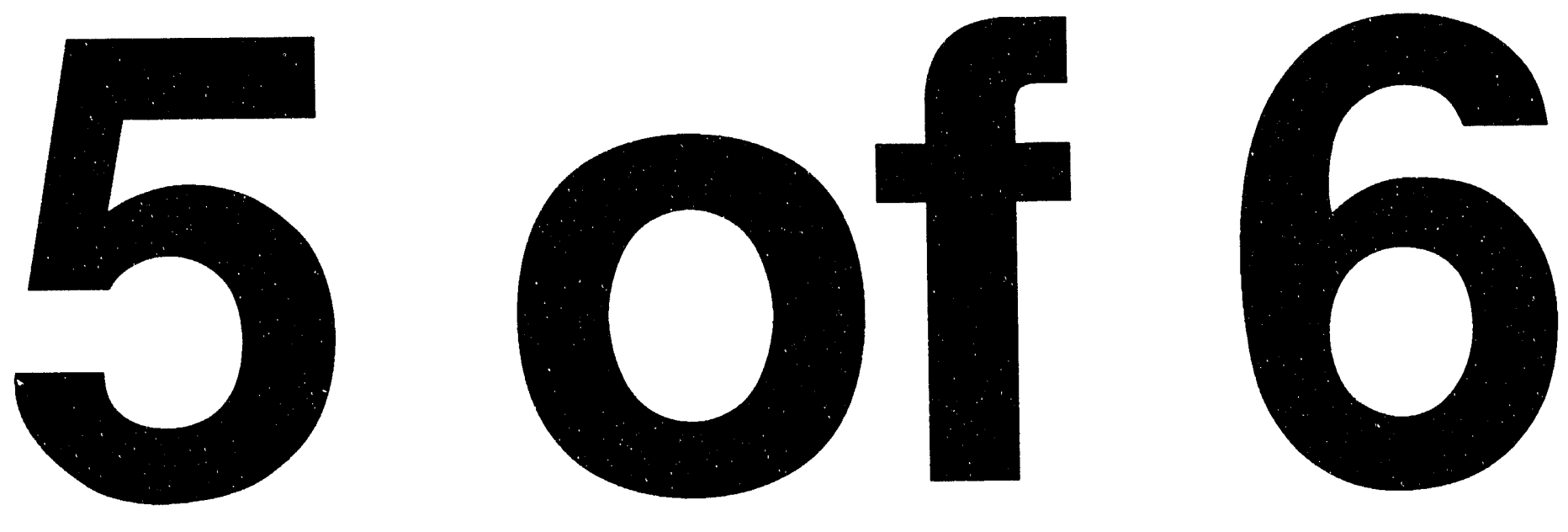


\section{Tank 241-T-103}

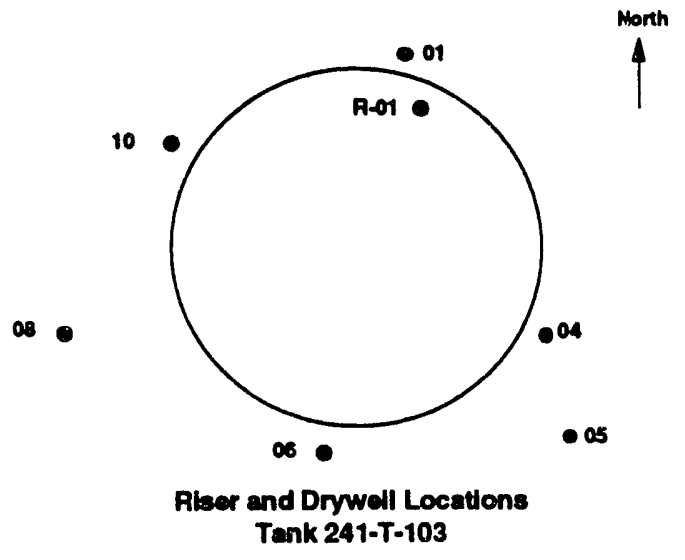

Number of External Drywells: 6

Number of Lateral Wells: None

\section{Tank Status}

\section{Contents}

Type: Non-Complexed Waste

Total Waste: $27 \mathrm{~K}$ gallons

Supernate Volume: $4 \mathrm{~K}$ gallons

Drainable Interstitial Liquid: OK gallons

Isolation Status

Date Interim Stabilized: $11 / 28 / 83$

Date Interim Isolated: $08 / 81$

\section{Surface Level/Leak Status}

Integrity Category: Assumed Leaker

Date Declared Confirmed or Assumed Leaker: 1974

Leak Volume Estimate: < 1.000 Gallons (1989)

FIC Surface Level: 7.10 Inches $(09 / 16 / 92)$

Last Photographed: 07/02/84

Photo Interpretation Comments:

Photographs from $4 / 2 / 81$ show more surface liquid from intrusions than previous photographs, and a band of exposed sludge around the perimeter of the tank. The FIC plummet is contacting liquid. Photographs from $7 / 3 / 84$ show little change from previous photographs

\section{iemperature Status}

Highest temperature during 1992: $64.6{ }^{\circ} \mathrm{F}(01 / 05 / 92)$

Comments: Temperatures are stable.

\section{Dryinell Status}

Comments: Current drywell profiles were stable and consistent with established baseline profiles. 
WHC-EP-0625

This page intentionally left blank. 


\section{Tank 241-T-104}

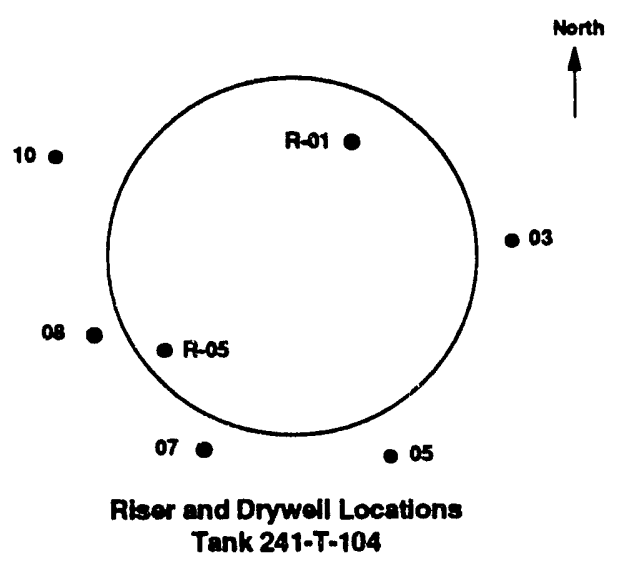

Number of External Drywells: 5

Number of Lateral Wells: None

\section{Tank Status}

\section{Contents}

Type: Non-Complexed Waste

Total Waste: $445 \mathrm{~K}$ gallons

Supernate Volume: $3 \mathrm{~K}$ gallons

Drainable Interstitial Liquid: $47 \mathrm{~K}$ gallons

Isolation Status

Date Partially Interim Isolated: $12 / 15 / 82$

\section{Surface Level/Leak Status}

Integrity Category: Sound

Manual Tape Surface Level: 155.00 Inches (12/28/92)

Last Photographed: 06/29/89

Photo Interpretation Comments:

Photographs from 3/18/81 reveal a surface of damp sludge, a larger band of liquid around the perimeter of the tank and a larger pool of liquid at the salt well than previous photographs. The manual tape pencil plummet is contacting sludge at the very edge of a small hole. Photographs from 08/02/84 appear to have slightly more liquid around the edge of the tank.

\section{Temperature Status}

Highest temperature during 1992: $70^{\circ} \mathrm{F}(11 / 05 / 92)$

Comments: Temperatures are stable.

\section{Drywell Status}

Comments: Current drywell profiles were stable and consistent with established baseline profiles.
Tank Description

Type: Single Shell

Constructed: 1944

In-service: $03 / 46$

Diameter: $75^{\circ}$

sable Depth: 16

city: $530 \mathrm{~K}$ gallons

Bottom shape: Dish

'

75.657' West

Ventilation: Passive

Surface Level:

FIC Riser- None

Manual Tape Riser- R-05

LOW Riser(s)- R-0 I

\section{Leak Detection System}


WHC-EP-0625

This page intentionally left blank. 


\section{Tank 241-T-105}

11

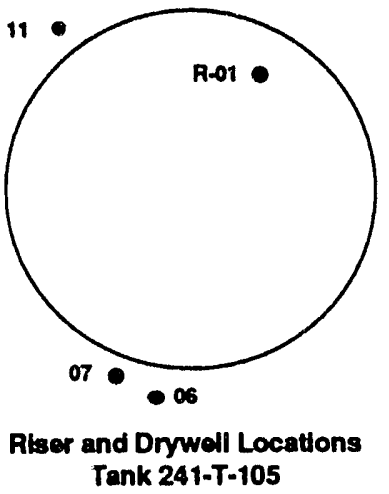

Number of External Drywells: 3 Number of Lateral Wells: None

Tank Status

Contents

Type: Non-Complexed Waste

Total Waste: $98 \mathrm{~K}$ gallons

Supernate Volume: OK gallons

Drainable Interstitial Liquid: $23 \mathrm{~K}$ gallons

Isolation Status

Date Interim Stabilized: 06/80

Date Partially Interim Isolated: 12/15/82

\section{Surface Level/Leak Status}

Integrity Category: Sound

FIC Surface Level: Intrusion level set at 30.10 Inches (12/28/92)

Surface Level Comments: Intrusion Mode

Last Photographed: 05/14/87

Photo Interpretation Comments:

Photographs show little change from those taken on 05/15/80. The surface appears to be damp solids with several pools and rivulets of liquid. The FIC plummet is contacting solids.

Temperature Status

Highest recent temperature: $75^{\circ} \mathrm{F}(02 / 07 / 81)$

Drywell Status

Comments: Current drywell profiles were stable and consistent with established baseline profiles.
Tank Description

Type: Single Shell

Constructed: 1944

In-service: $07 / 46$

Out of Service: 1974

Diameter: $75^{\circ}$

Usable Depth: 16'

Capacity: $530 \mathrm{~K}$ gallons

Bottom shape: Dish

Hanford Coordinates: $43,547^{\prime}$ North 75,737' West

Ventilation: Passive

\section{Leak Detection System}

Surface Level:

FIC Riser- R-01

Manual Tape Riser- None

LOW Riser(s)- None 
WHC-EP-0625

This page intentionally left blank. 


\section{Tank 241-T-106}

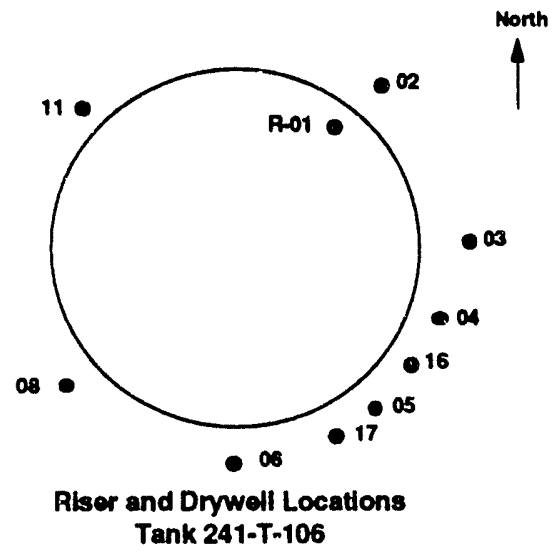

Number of External Drywells: 9

Number of Lateral Wells: None
Tank Description

Type: Single Shell

Constructed: 1944

In-service: $06 / 47$

Out of Service: 1973

Diameter: $75^{\circ}$

Usable Depth: $16^{\prime}$

Capactty: $530 \mathrm{~K}$ gallons

Bottom shape: Dish

Hanford Coordinates: $43,547^{\prime}$ North 75,837' West

Ventilation: Passive

\section{Leak Detection System}

Surface Level:

FIC Riser- R-01

Manual Tape Riser- None

LOW Riser(s)- None

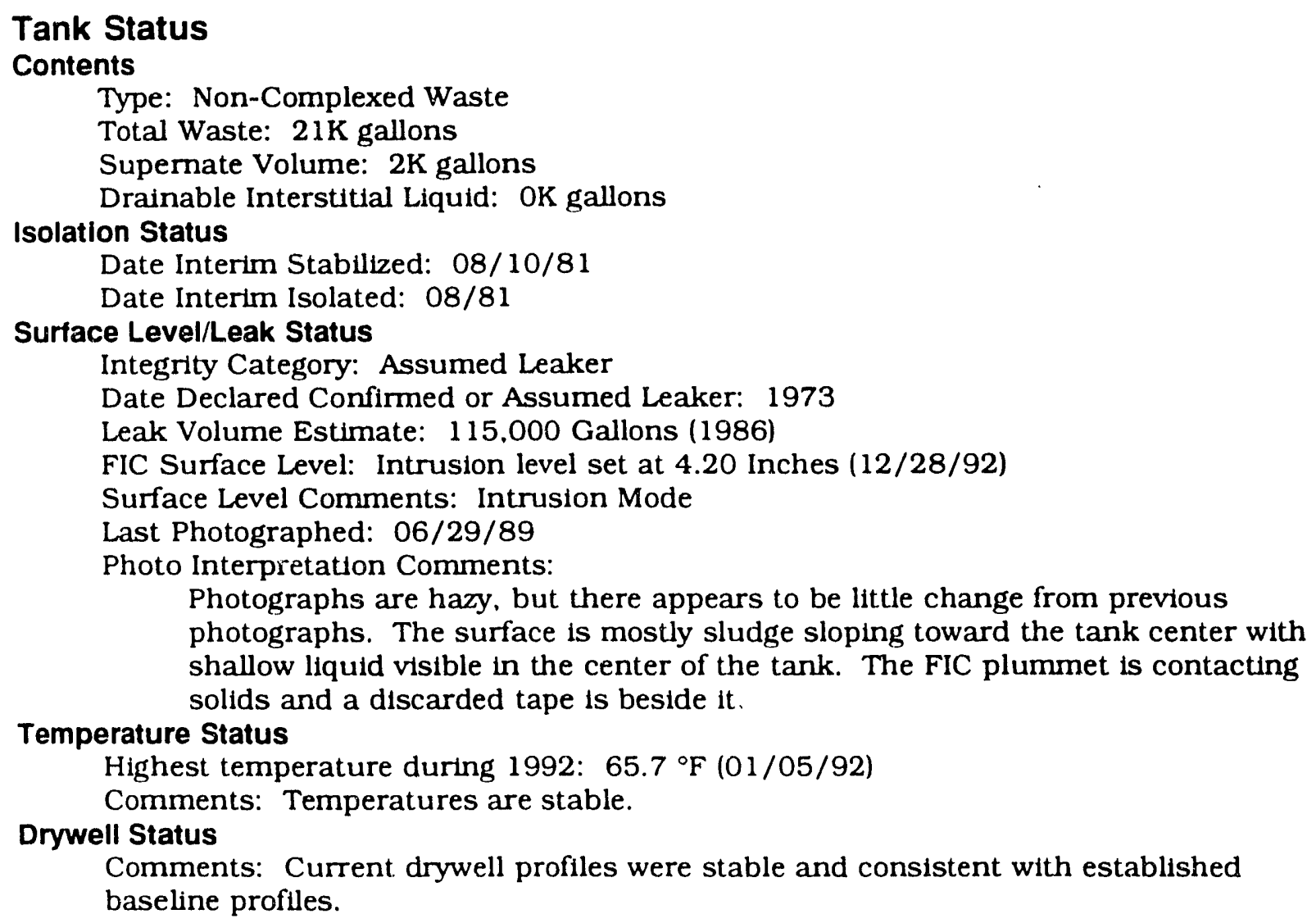

Comments: Current drywell profiles were stable and consistent with established baseline profiles. 


\section{WHC-EP-0625}

This page intentionally left blank. 


\section{Tank 241-T-107}

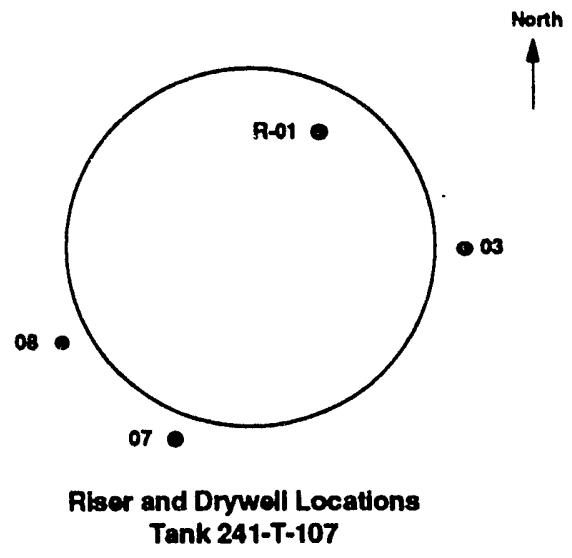

Number of External Drywells: 3

Number of Lateral Wells: None

Tank Status

Watch List: Ferrocyanide

Contents

Type: Non-Complexed Waste

Total Waste: 130K gallons

Supernate Volume: 9K gallons

Drainable Interstitial Liquid: $13 \mathrm{~K}$ gallons

Isolation Status

Date Partially Interim Isolated: $12 / 15 / 82$

Surface Level/Leak Status

Integrity Category: Assumed Leaker

Date Declared Confirmed or Assumed Leaker: 1984

Leak Volume Estimate: $7.8 \mathrm{~K}$ gallons $=1 / 19$ th of $150 \mathrm{~K}$ gallons

FIC Surface Level: 66.50 Inches (12/28/92)

Last Photographed: $07 / 12 / 84$

Photo Interpretation Comments: See attached photo.

Temperature Status

Highest temperature during 1992: $73^{\circ} \mathrm{F}(10 / 07 / 92)$

Comments: Temperatures are stable.

Drywell Status

Comments: Current drywell profiles were stable and consistent with established baseline proflles.
Tank Description

Type: Single Shell

Constructed: 1944

In-service: $12 / 44$

Out of Service: 1976

Diameter: $75^{\circ}$

Usable Depth: 16'

Capacity: $530 \mathrm{~K}$ gallons

Bottom shape: Dish

Hanford Coordinates:

$43,447^{\prime}$ North

75,637' West

Ventilation: Passive

Surface Level:

FIC Riser- R-01

Manual Tape Riser- None

LOW Riser(s)- None

\section{Leak Detection System}


WHC-EP-0625

This page intentionally left blank. 
WHC-EP-0625

\section{Tank: 241-T-107}

July 1984




WHC-EP-0625

This page intentionally left blank. 


\section{Tank 241-T-108}

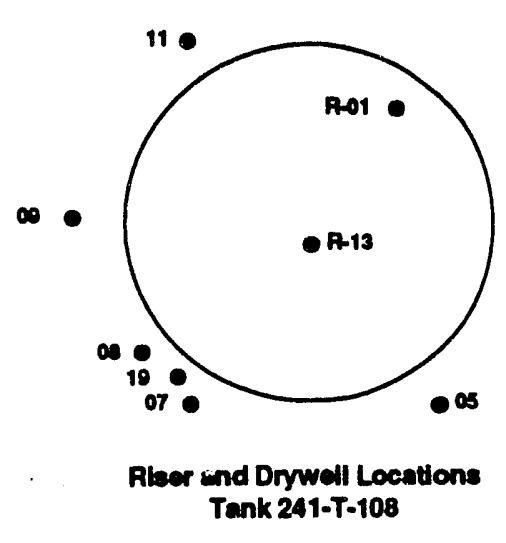

Number of External Drywells: 6

Number of Lateral Wells: None
Tank Description

Type: Single Shell

Constructed: 1944

In-service: 09/45

Out of Service: 04/47

Diameter: $7^{\circ}$

Usable Depth: 16'

Capacity: $530 \mathrm{~K}$ gallons

Bottom shape: Dish

Hanford Coordinates:

43.447 North

75,737' West

Ventilation: Passive

\section{Leak Detection System}

Surface Level:

FIC Riser- R-01

Manual Tape Riser- R-13

LOW Riser(s)- None

\section{Tank Status \\ Contents}

Type: Non-Complexed Waste

Total Waste: $44 \mathrm{~K}$ gallons

Supernate Volume: OK gallons

Drainable Interstitial Liquid: OK gallons

Isolation Status

Date Interim Stabilized: $11 / 30 / 78$

Date Interim Isolated: 06/81

Surface Level/Leak Status

Integrity Category: Assumed Leaker

Date Declared Confirmed or Assumed Leaker: 1974

Leak Volume Estimate: < 1,000 Gallons (1980)

FIC Surface Level: 13.00 Inches (10/01/92)

Last Photographed: $07 / 17 / 84$

Photo Interpretation Comments:

Photographs show a surface that appears to be mostly damp sludge with the manual tape plummet over a clear liquid pool ( 7 to 10 feet in diameter).

\section{Temperature Status}

Highest temperature during 1992: $70^{\circ} \mathrm{F}(02 / 07 / 81)$

Drywell Status

Comments: Current drywell profiles were stable and consistent with established baseline profiles. 


\section{WHC-EP-0625}

This page intentionally left blank. 


\section{Tank 241-T-109}

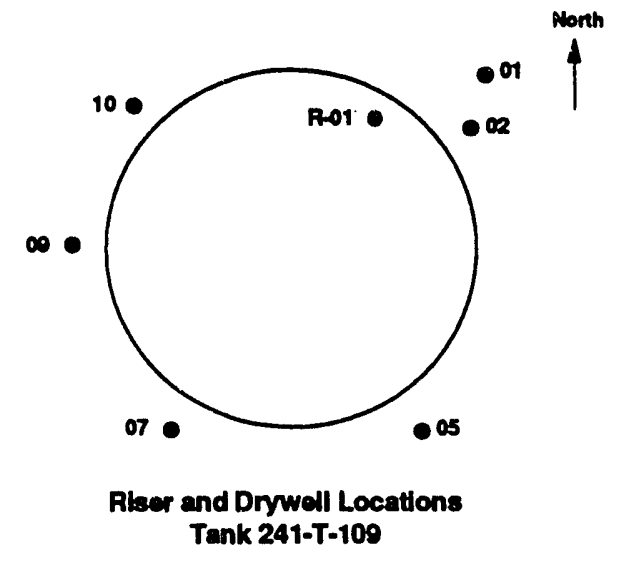

Number of External Drywells: 6

Number of Lateral Wells: None

\section{Tank Status \\ Contents}

Type: Non-Complexed Waste

Total Waste: $58 \mathrm{~K}$ gallons

Supernate Volume: OK gallons

Drainable Interstitial Liquid: oK gallons

Isolation Status

Date Interim Stabilized: 12/20/84

Date Partially Interim Isolated: $12 / 15 / 82$

\section{Surface Level/Leak Status}

Integrity Category: Assumed Leaker

Date Declared Confirmed or Assumed Leaker: 1974

Leak Volume Estimate: < 1.000 Gallons (1989)

FIC Surface Level: Intrusion level set at 16.70 Inches (12/21/92)

Surface Level Comments: Intrusion Mode

Last Photographed: 07/03/84

Photo Interpretation Comments:

Photographs reveal an uneven surface of sludge with no visible liquid. The FIC plummet is not visible but would be contacting solids in a depression and measurement anomalies should be expected.

Temperature Status

Highest recent temperature: $75^{\circ} \mathrm{F}(02 / 16 / 91)$

\section{Drywell Status}

Comments: Current drywell profiles were stable and consistent with established baseline profiles.
Tank Description

Type: Single Shell

Constructed: 1944

Cinice: $12 / 45$

Diameter: $75^{\circ}$

Usable Depth: 16'

Capacity: $530 \mathrm{~K}$ gallons

Bottom shape: Dish

Hanford Coordinates: $43,447^{\prime}$ North 75,837' West

Ventilation: Passive

\section{Leak Detection System}

Surface Level:

FIC Riser- R-01

Manual Tape Riser- None LOW Riser(s)- None 


\section{WHC-EP-0625}

This page intentionally left blank. 


\section{Tank 241-T-110}

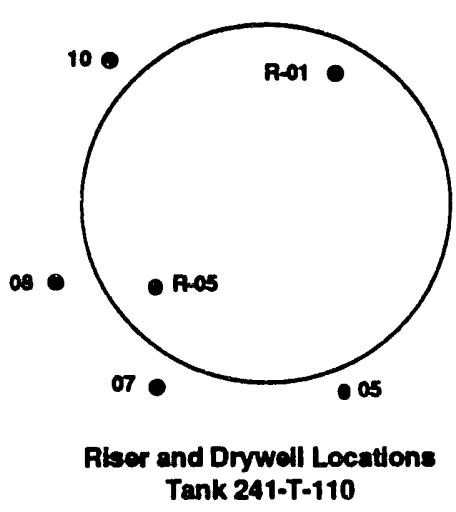

1

Number of External Drywells: 4

Number of Lateral Wells: None

\section{Tank Status}

\section{Watch List: Hydrogen}

Contents

Type: Non-Complexed Waste

Total Waste: $379 \mathrm{~K}$ gallons

Supernate Volume: $3 \mathrm{~K}$ gallons

Drainable Interstitial Liquid: $39 \mathrm{~K}$ gallons

Isolation Status

Date Partially Interim Isolated: $12 / 15 / 82$

\section{Surface Level/Leak Status}

Integrity Category: Sound

FIC Surface Level: 147.70 Inches (12/28/92)

Last Photographed: $07 / 12 / 84$

Photo Interpretation Comments: See attached photo.

Temperature Status

Highest temperature during 1992: $68.5^{\circ} \mathrm{F}(09 / 10 / 92)$

Comments: Temperatures are stable.

\section{Drywell Status}

Comments: Current drywell profiles were stable and consistent with established baseline proflles.
Tank Description

Type: Single Shell

Constructed: 1944

In-service: $12 / 44$

Diameter: $7^{\circ}$

Usable Depth: $16^{\prime}$

Capacity: $530 \mathrm{~K}$ gallons

Bottom shape: Dish

Hanford Coordinates:

43,347' North

75,637' West

Ventulation: Passive

\section{Leak Detection System}

Surface Level:

FIC Riser- R-01

Manual Tape Riser- None

LOW Riser(s)- R-05 
WHC-EP-0625

This page intentionally left blank. 


\title{
Tank: 241-T-110
}

\author{
fuly 198.1
}

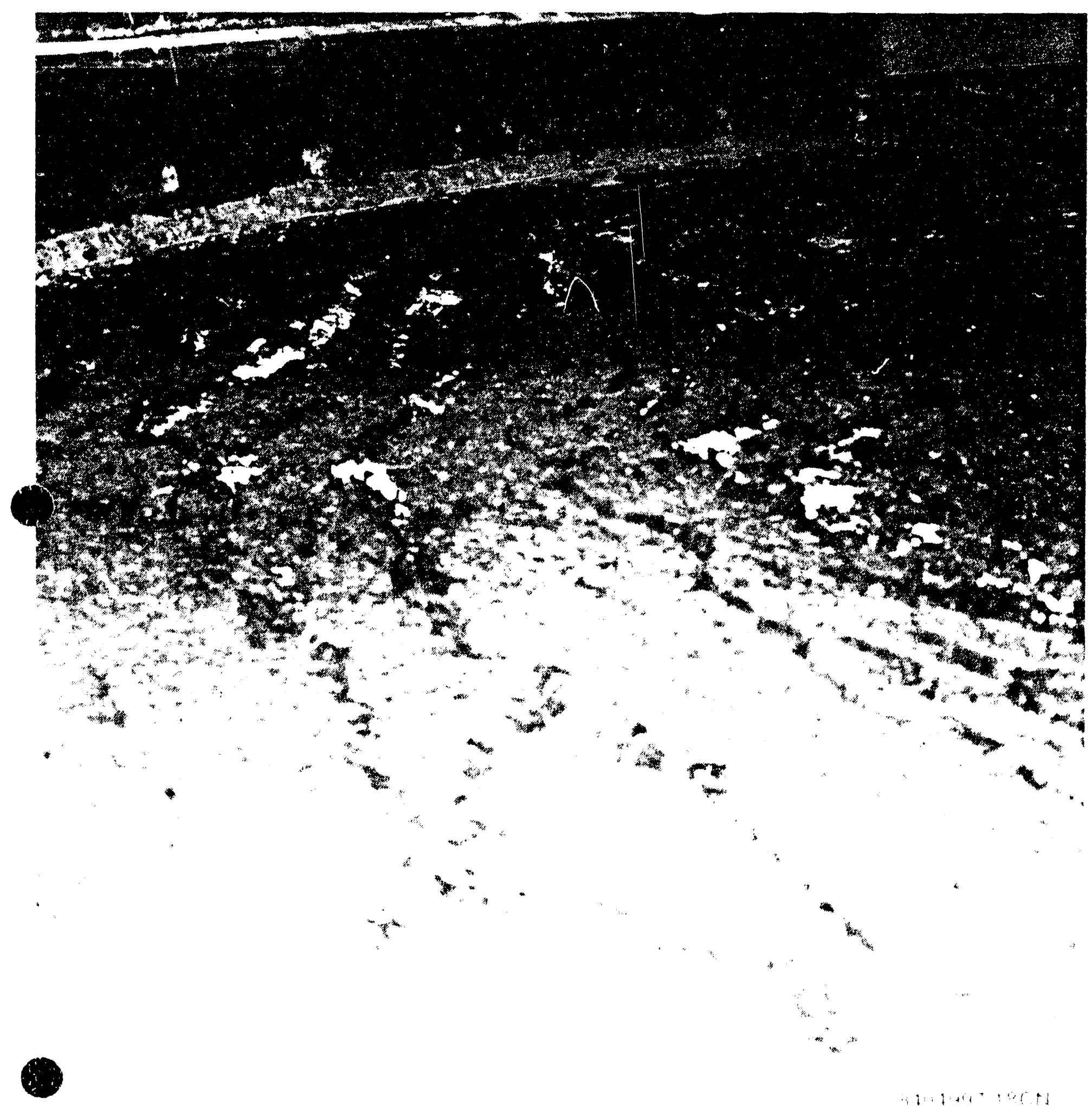


WHC-EP-0625

This page intentionally left blank. 


\section{Tank 241-T-111}

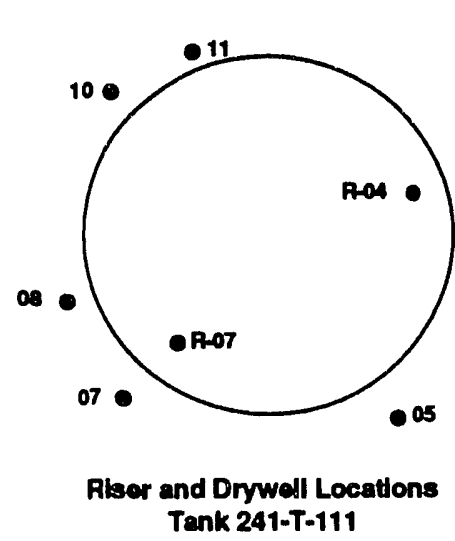

Number of External Drywells: 5 Number of Lateral Wells: None
Tank Description Type: Single Shell Constructed: 1944 In-service: $10 / 45$ Out of Service: $04 / 74$ Diameter: $7^{\circ}$ Usable Depth: 16' Capacity: $530 \mathrm{~K}$ gallons Bottom shape: Dish Hanford Coordinates: 43,347 North 75.737' West Ventilation: Passive

\section{Leak Detection System}

Surface Level: FIC Riser- R-04 Manual Tape Riser- None LOW Riser(s)- R-07

\section{Tank Status}

Contents

Type: Non-Complexed Waste Total Waste: $458 \mathrm{~K}$ gallons Supernate Volume: $2 \mathrm{~K}$ gallons Drainable Interstitial Liquid: $49 \mathrm{~K}$ gallons Isolation Status

Date Interim Stabilized: 09/79

Date Partially Interim Isolated: 12/15/82

Surface Level/Leak Status Integrity Category: Assumed Leaker Date Declared Confirmed or Assumed Leaker: 1984 Leak Volume Estimate: < 1.000 Gallons (1980) FIC Surface Level: 162.60 Inches (12/14/92) Last Photographed: 08/02/84

Photo Interpretation Comments:

Photographs from $1 / 30 / 81$ show a flat, cracked, flagstone-like surface of sludge and a larger liquid pool around the salt well than previous photos. The FIC plummet is contacting a very small puddle of liquid in a depression caused by repeated plummet flushes. Photograph from 08/01/84 comparisons show little change if any.

\section{Temperature Status}

Highest temperature during 1992: $71.8^{\circ} \mathrm{F}(01 / 05 / 92)$

Comments: Temperatures are stable.

Drywell Status

Comments: Current drywell profiles were stable and consistent with established baseline profiles. 


\section{WHC-EP-0625}

This page intentionally left blank. 


\section{Tank 241-T-112}

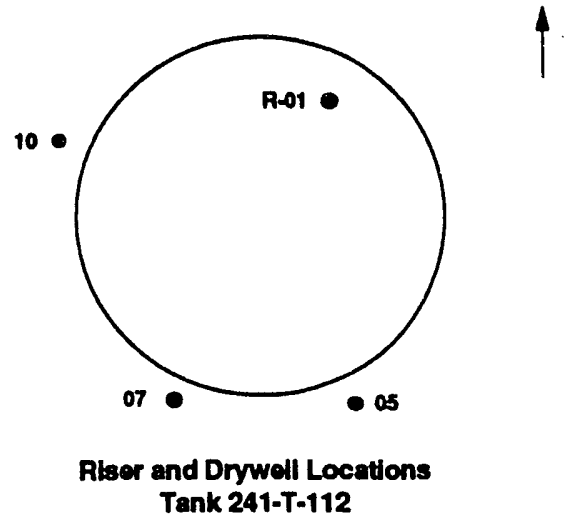

Number of External Drywells: 3 Number of Lateral Wells: None

\section{Tank Status}

Contents

Type: Non-Complexed Waste

Total Waste: $67 \mathrm{~K}$ gallons

Supernate Volume: $7 \mathrm{~K}$ gallons

Drainable Interstitial Liquid: OK gallons

Isolation Status

Date Interim Stabilized: $03 / 81$

Date Interim Isolated: $06 / 81$

\section{Surface Level/Leak Status}

Integrity Category: Sound

FIC Surface Level: 20.30 Inches $(12 / 28 / 92)$

Last Photographed: 08/01/84

Photo Interpretation Comments:

Photographs show a surface of exposed sludge and liquid. The FIC plummet is contacting clear liquid.

\section{Temperature Status}

Highest temperature during 1992: $70^{\circ} \mathrm{F}(11 / 05 / 92)$

Comments: Temperatures are stable.

Drywell Status

Comments: Current drywell profiles were stable and consistent with established baseline proflles.
Tank Description

Type: Single Shell

Constructed: 1944

In-service: $01 / 46$

Diameter: $75^{\prime}$

Usable Depth: 16'

Capacity: $530 \mathrm{~K}$ gallons

Bottom shape: Dish

Hanford Coordinates:

43,347' North

75,837' West

Ventilation: Passive

\section{Leak Detection System}

Surface Level:

FIC Riser- R-01

Manual Tape Riser- None

LOW Riser(s)- None 


\section{WHC-EP-0625}

This page intentionally left blank. 
WHC-EP-0625

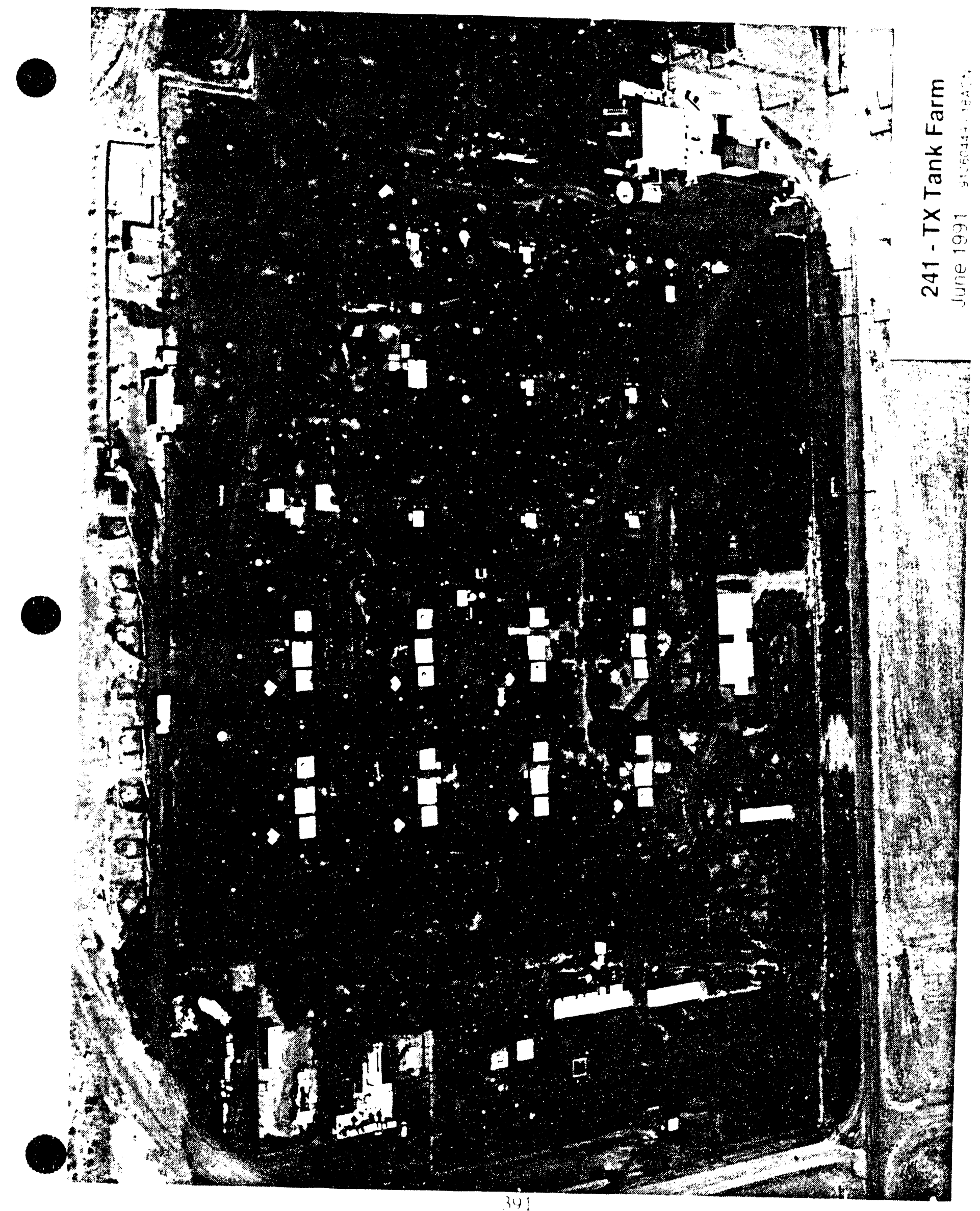


WHC-EP-0625

This page intentionally left blank. 


\section{Tank 241-TX-101}

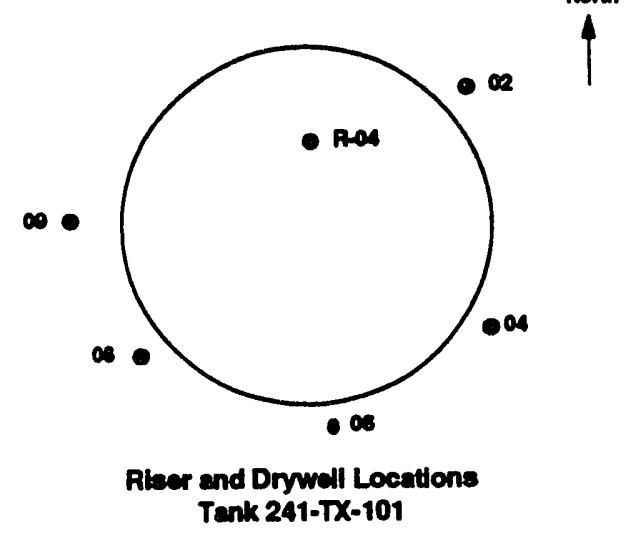

Number of External Drywells: 5

Number of Lateral Wells: None

\section{Tank Status}

Contents

Type: Non-Complexed Waste

Total Waste: $87 \mathrm{~K}$ gallons

Supernate Volume: 3K gallons

Drainable Interstitial Liquid: $2 \mathrm{~K}$ gallons

Isolation Status

Date Interim Stabilized: 02/03/84

Date Interim Isolated: $08 / 84$

Surface Level/Leak Status

Integrity Category: Sound

FIC Surface Level: 40.30 Inches (12/28/92)

Last Photographed: 10/24/85

Photo Interpretation Comments:

Photographs from $01 / 31 / 84$ show a shallow liquid surface and exposed solids around the tank perimeter. The FIC plummet is contacting a small island of sludge. Photographs of the surface area from 10/24/85 are not clear.

Temperature Status

Highest temperature during 1992:

\section{Drywell Status}

Comments: Current drywell profiles were stable and consistent with established baseline profiles.
Tank Description

Type: Single Shell

Constructed: 1948

In-service: $07 / 27 / 49$

Dlameter: $7^{\circ}$

Usable Depth: 23'

Capacity: $758 \mathrm{~K}$ gallons

Bottom shape: Dish

Hanford Coordinates:

$41,650^{\circ}$ North

$75.750^{\circ}$ West

Ventilation: Passive

\section{Leak Detection System \\ Surface Level: \\ FIC Riser- R-04 \\ Manual Tape Riser- None \\ LOW Riser(s)- None}


WHC-EP-0625

This page intentionally left blank. 


\section{Tank 241-TX-102}

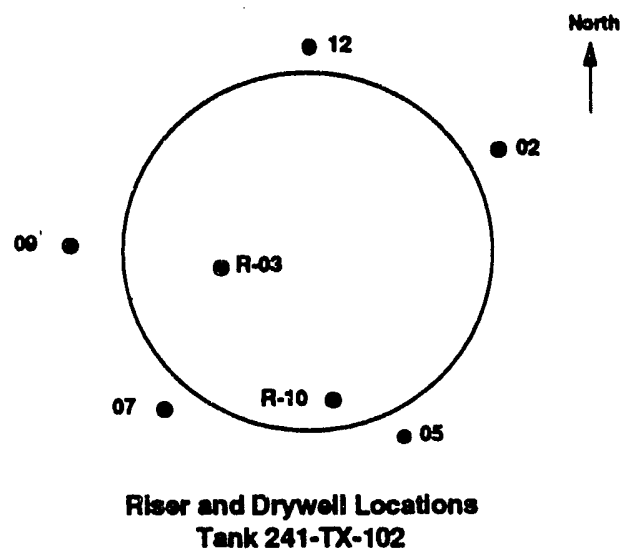

Number of External Drywells: 5

Number of Lateral Wells: None

\section{Tank Status}

Contents

Type: Non-Complexed Waste

Total Waste: $113 \mathrm{~K}$ gallons

Supernate Volume: OK gallons

Drainable Interstitial Liquid: $22 \mathrm{~K}$ gallons

Isolation Status

Date Interim Stabilized: 04/05/83

Date Interim Isolated: $08 / 84$

\section{Surface Level/Leak Status}

Integrity Category: Sound

Manual Tape Surface Level: 82.50 Inches (12/21/92)

Last Photographed: 10/31/85

Photo Interpretation Comments:

Photographs from $11 / 10 / 82$ show a crusted uneven surface of salt cake with no visible liquid. The salt cake has slumped toward the tank center and the pencil plummet is contacting solids in a deep depression. Measurement anomalies can be expected. Photographs from 10/31/85 show little or no change.

\section{Temperature Status}

Highest temperature during 1992:

\section{Drywell Status}

Comments: Current drywell profiles were stable and consistent with established baseline profiles. 
WHC-EP-0625

This page intentionally left blank. 


\section{Tank 241-TX-103}

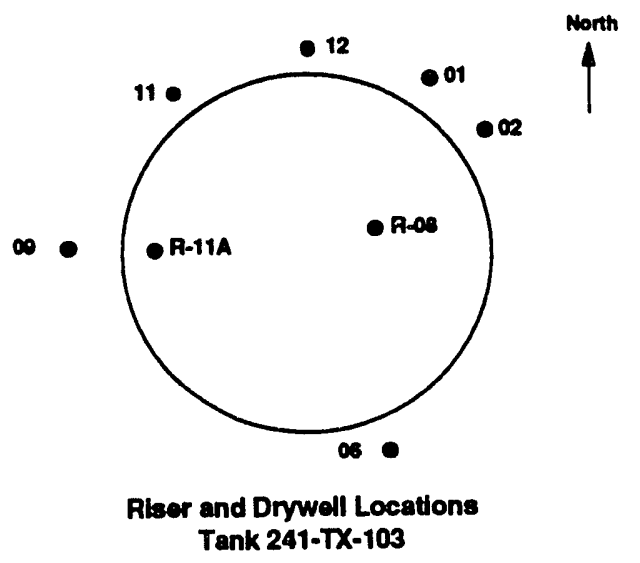

Number of External Drywells: 6 Number of Lateral Wells: None

\section{Tank Status}

Contents

Type: Non-Complexed Waste

Total Waste: $157 \mathrm{~K}$ gallons

Supernate Volume: OK gallons

Drainable Interstitial Liquid: $15 \mathrm{~K}$ gallons

Isolation Status

Date Interim Stabilized: $08 / 12 / 83$

Date Interim Isolated: $08 / 84$

\section{Surface Level/Leak Status}

Integrity Category: Sound

FIC Surface Level: Intrusion level set at 58.60 Inches $(12 / 28 / 92)$

Surface Level Comments: Intrusion Mode

Last Photographed: 10/31/85

Photo Interpretation Comments:

Photographs show what appear to be soft moist solids with isolated pockets of liquid and a small pool of liquid in the vicinity of the salt well. The FIC plummet is above solids.

\section{Temperature Status}

Highest temperature during 1992:

\section{Drywell Status}

Comments: Current drywell profiles were stable and consistent with established baseline profiles. 
WHC-EP-0625

This page intentionally left blank. 


\section{Tank 241-TX-104}

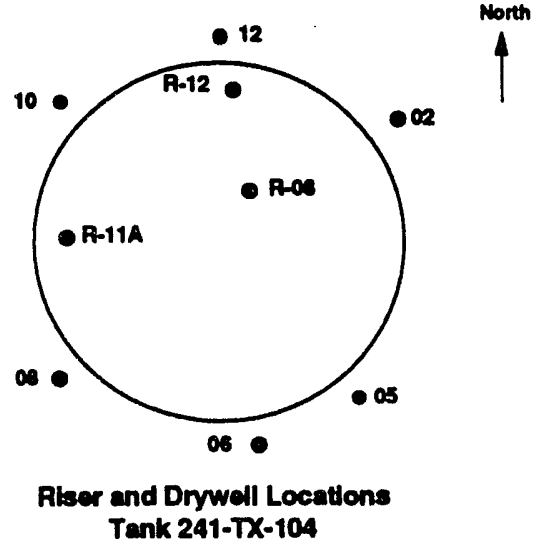

Number of External Drywells: 6 Number of Lateral Wells: None
Tank Description

Type: Single Shell

Constructed: 1948

In-service: $11 / 12 / 50$

Diameter: $75^{\prime}$

Usable Depth: 23'

Capacity: $758 \mathrm{~K}$ gallons

Bottom shape: Dish

Hanford Coordinates:

$41,650^{\prime}$ North

76,056' West

Ventilation: Passive

\section{Leak Detection System \\ Surface Level:}

FIC Riser- R-08

Manual Tape Riser- R-11A

LOW Riser(s)- None

\section{Tank Status \\ Contents}

Type: Non-Complexed Waste

Total Waste: $65 \mathrm{~K}$ gallons

Supernate Volume: $1 \mathrm{~K}$ gallons

Drainable Interstitial Liquid: $14 \mathrm{~K}$ gallons

Isolation Status

Date Interim Stabilized: $09 / 18 / 79$

Date Interim Isolated: $08 / 84$

Surface Level/Leak Status

Integrity Category: Sound

FIC Surface Level: 32.20 Inches $(12 / 28 / 92)$

Last Photographed: $10 / 16 / 84$

Photo Interpretation Comments:

Photographs show surface liquid in the vicinity of the tank center and an uneven surface of exposed solids (going from damp to dry) toward the outer edge.

Photograph comparisons (04/03/84 photographs) tend to show a shifting of solids and a larger but shallower pool of liquid at the tank center. The FIC plummet appears to be contacting liquid.

Temperature Status

Highest temperature during 1992: $82.6^{\circ} \mathrm{F}(07 / 04 / 92)$

Comments: Temperatures are stable.

Drywell Status

Comments: Current drywell profiles were stable and consistent with established baseline profiles. 
WHC-EP-0625

This page intentionally left blank. 


\section{Tank 241-TX-105}

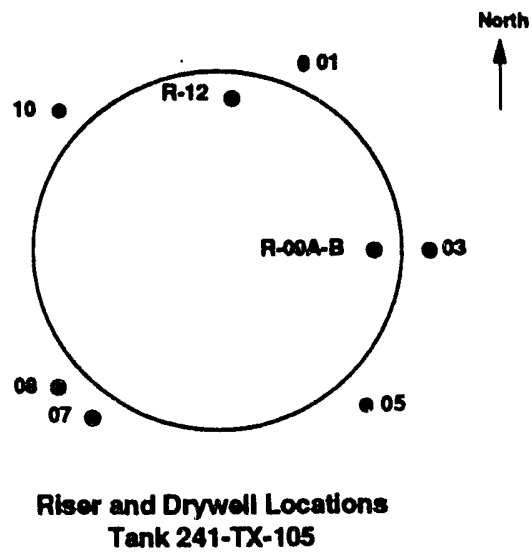

Number of External Drywells: 6

Number of Lateral Wells: None

\section{Tank Status}

Watch LIst: Organic Salts

Contents

Type: Non-Complexed Waste

Total Waste: $609 \mathrm{~K}$ gallons

Supernate Volume: OK gallons

Drainable Interstitial Liquid: $20 \mathrm{~K}$ gallons

Isolation Status

Date Interim Stabilized: 09/29/83

Date Interim Isolated: $08 / 84$

\section{Surface Level/Leak Status}

Integrity Category: Assumed Leaker

Date Declared Confirmed or Assumed Leaker: 1977

Leak Volume Estimate: $7.8 \mathrm{~K}$ gallons $=1 / 19$ th of $150 \mathrm{~K}$ gallons

Manual Tape Surface Level: 214.00 Inches (10/01/92)

Last Photographed: $10 / 24 / 89$

Photo Interpretation Comments:

Photographs show a very irregular, broken, crusted surface of salt cake with many

\section{Temperature Status} deep holes. The M-tape plummet is contacting solids in a depression.

Highest temperature during 1992: $104^{\circ} \mathrm{F}(04 / 23 / 92)$

Comments: Temperatures are stable.

\section{Drywell Status}

Comments: Current drywell profiles were stable and consistent with established baseline profiles. 
WHC-EP-0625

This page intentionally left blank. 
WHC-EP-0625

\section{Tank: 241-TX-105}

\section{October 1929}

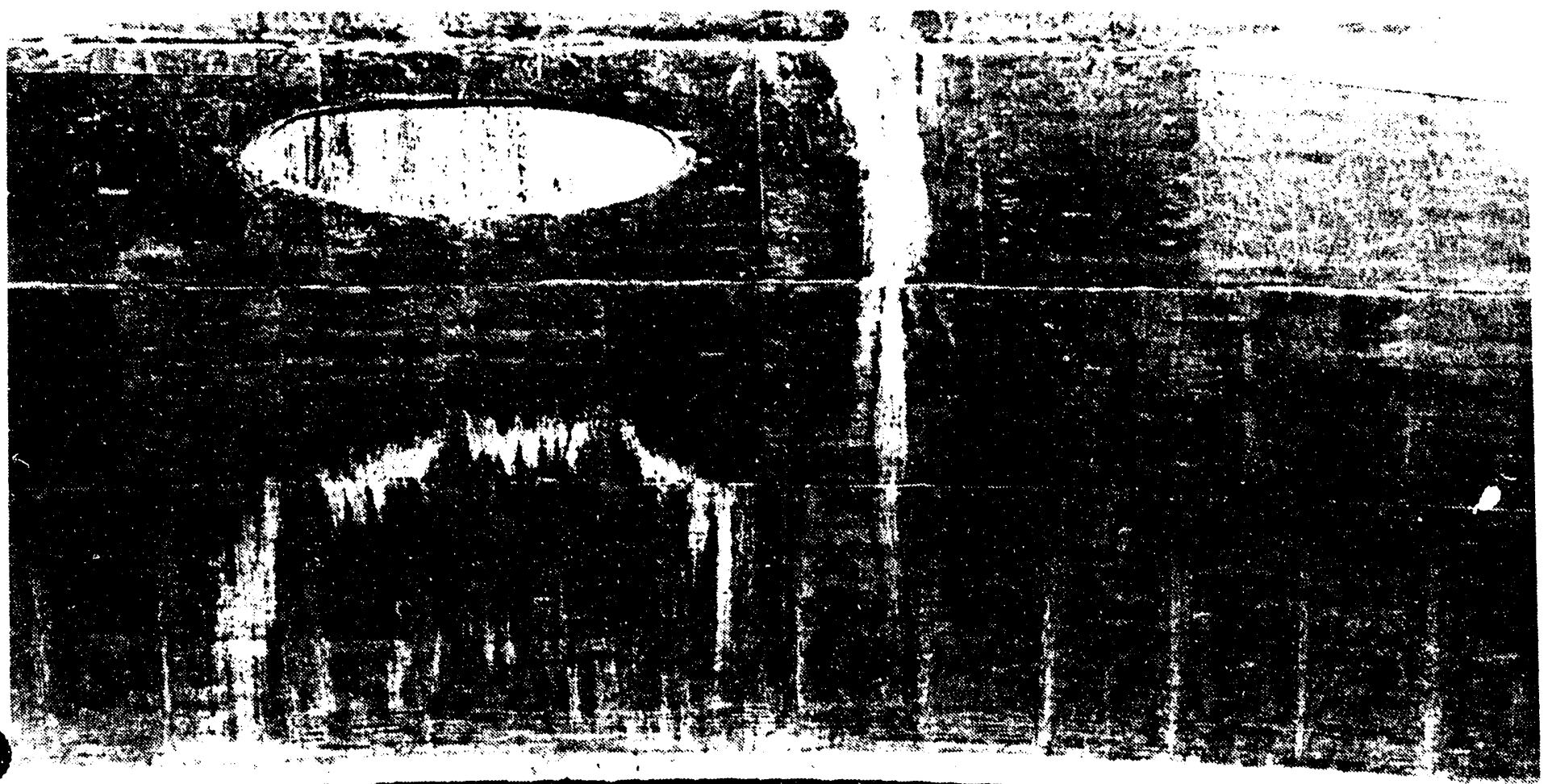

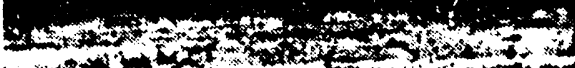

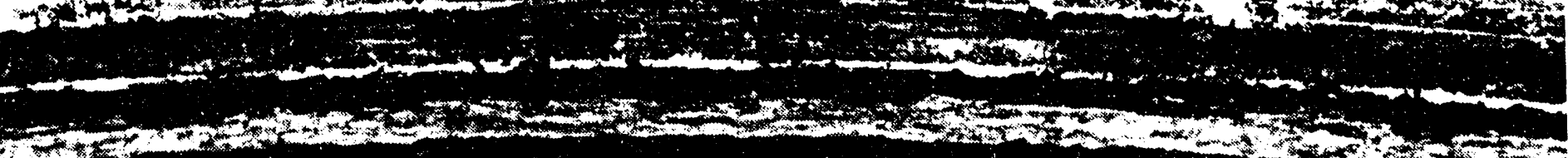
-

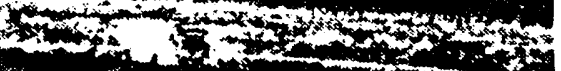

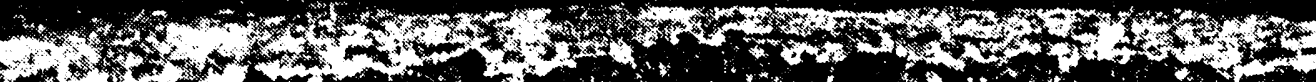

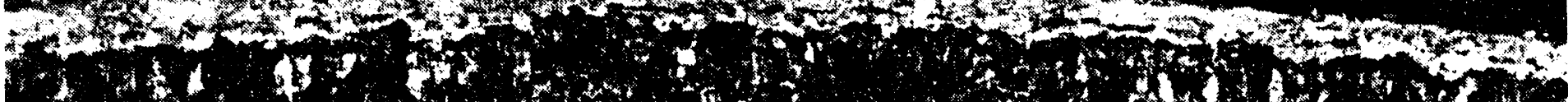

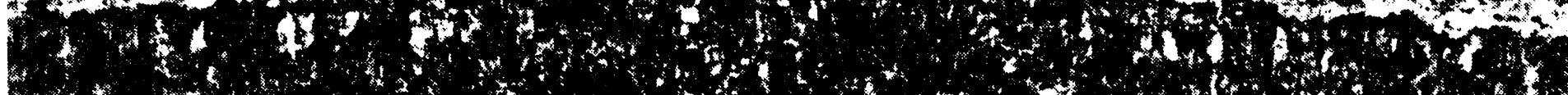

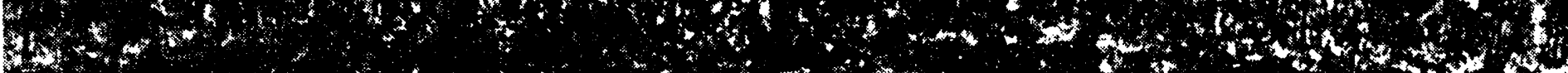

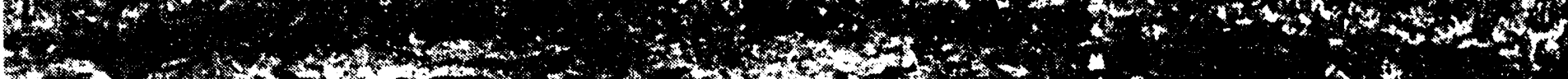

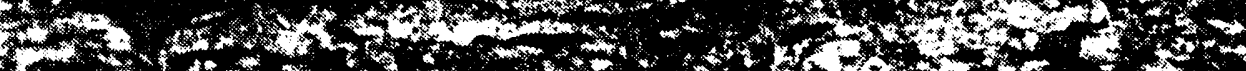

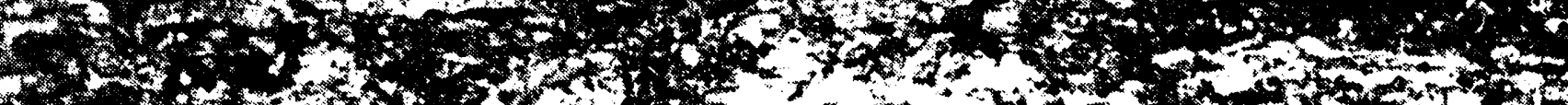

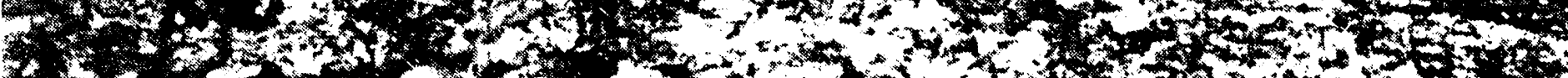

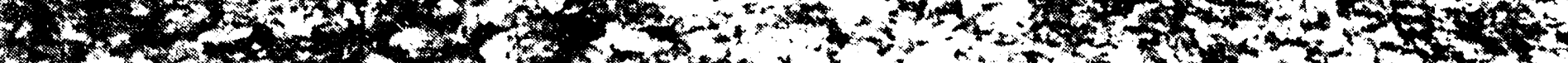

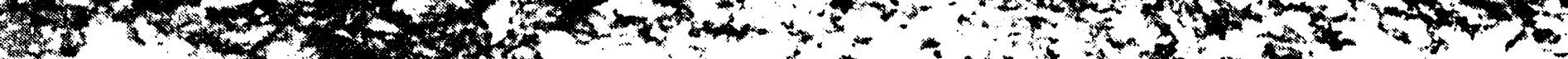

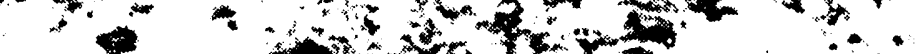

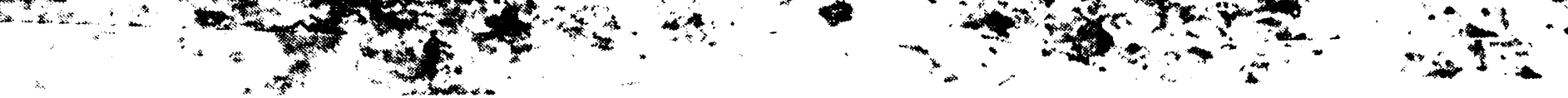


WHC-EP-0625

This page intentionally left blank. 


\section{Tank 241-TX-106}

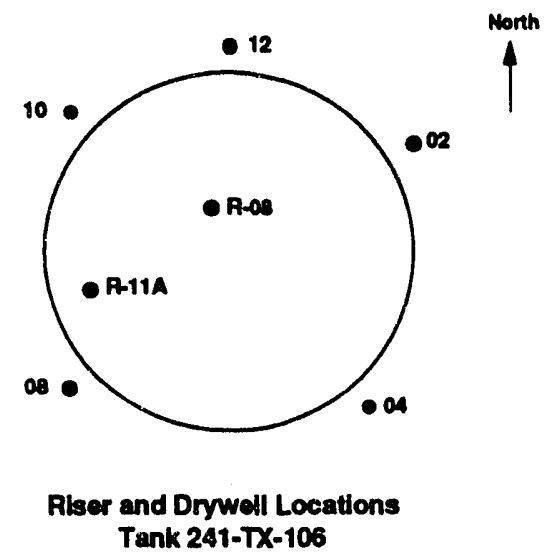

Number of External Drywells: 5

Number of Lateral Wells: None

\section{Tank Status}

\section{Contents}

Type: Non-Complexed Waste

Total Waste: $453 \mathrm{~K}$ gallons

Supernate Volume: OK gallons

Drainable Interstitial Liquid: $10 \mathrm{~K}$ gallons

Isolation Status

Date Interim Stabilized: 06/08/83

Date Interim Isolated: $08 / 84$

\section{Surface Level/Leak Status}

Integrity Category: Sound

Manual Tape Surface Level: 133.50 Inches (10/01/92)

Last Photographed: $10 / 31 / 85$

Photo Interpretation Comments:

Photographs from 06/09/82 and 10/31/85 show a crusted, uneven surface, that is free of liquid. The manual tape plummet is contacting solids and a discarded tape is near the plummet.

\section{Temperature Status}

Highest temperature during 1992:

\section{Drywell Status}

Comments: Current drywell profiles were stable and consistent with established baseline profiles.
Tank Description

Type: Single Shell

In-service: $06 / 16 / 51$

Diameter: $75^{\prime}$

Usable Depth: 23'

Capacity: 758K gallons

Bottom shape: Dish

Hanford Coordinates:

41.752' North

75,852' West

Ventilation: Passive

\section{Leak Detection System}

Surface Level:

FIC Riser- None

Manual Tape Riser- R-08

LOW Riser(s)- R-1 1A
Constructed: 1948 
WHC-EP-0625

This page intentionally left blank. 


\section{Tank 241-TX-107}

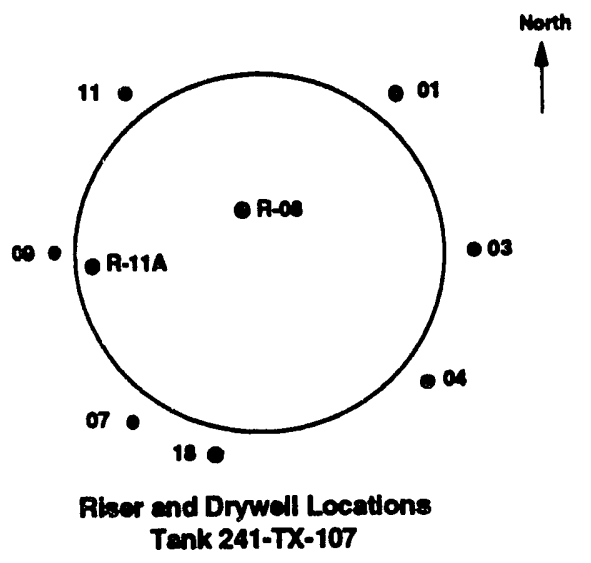

Number of External Drywells: 7

Number of Lateral Wells: None

\section{Tank Status}

Contents

Type: Non-Complexed Waste

Total Waste: $36 \mathrm{~K}$ gallons

Supernate Volume: $1 \mathrm{~K}$ gallons

Drainable Interstitial Liquid: $1 \mathrm{~K}$ gallons

Isolation Status

Date Interim Stabilized: $10 / 79$

Date Interim Isolated: $08 / 84$

Surface Level/Leak Status

Integrity Category: Assumed Leaker

Date Declared Confirmed or Assumed Leaker: 1984

Leak Volume Estimate: 2,500 Gallons (1986)

FIC Surface Level: 17.70 Inches $(12 / 28 / 92)$

Last Photographed: 10/31/85

Photo Interpretation Comments:

Photographs from 07/13/84 show exposed solids and a liquid pool in the center.

The FIC plummet appears to be contacting liquid, but is not readily visible.

Photographs from 10/31/85 show little change. although there does appear to be

Temperature Status

slightly less liquid.

Highest temperature during 1992:

\section{Dry:-ell Status}

Comments: Current drywell proflles were stable and consistent with established baseline profiles.
Tank Description

Type: Single Shell

Constructed: 1948

In-service: 1950

Diameter: $7^{\circ}$

Bottom shape: Dish

Hanford Coordinates:

$41,752^{\prime}$ North

75,954' West

Ventilation: Passtve

\section{Leak Detection System}

Surface Level:

FIC Riser- R-08

Manual Tape Riser- R-11A

LOW Riser(s)- None
Usable Depth: 23'

Capactty: $758 \mathrm{~K}$ gallons 
WHC-EP-0625

This page intentionally left blank. 


\section{Tank 241-TX-108}

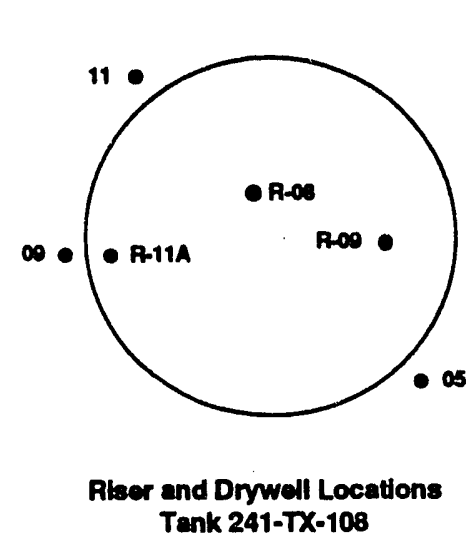

North

Number of External Drywells: 3

Number of Lateral Wells: None

\section{Tank Status}

\section{Contents}

Type: Non-Complexed Waste

Total Waste: $134 \mathrm{~K}$ gallons

Supernate Volume: OK gallons

Drainable Interstitial Liquid: OK gallons

Isolation Status

Date Interim Stabilized: $03 / 28 / 83$

Date Interim Isolated: $08 / 84$

\section{Surface Level/Leak Status}

Integrity Category: Sound

FIC Surface Level: Intrusion level set at 57.10 Inches (12/28/92)

Surface Level Comments: Intrusion Mode

Last Photographed: 09/12/89

Photo Interpretation Comments:

Photographs from $05 / 29 / 81$ show a shallow liquid surface continuing to increase in size (no surface liquid in 04/04/77 photographs). Photographs from 04/12/83 show the FIC and manual tape plummet contacting mounds of exposed salt cake

Temperature Status with no visible liquid.

Highest temperature during 1992:

Drywell Siatus

Comments: Current drywell profiles were stable and consistent with established baseline profiles. 


\section{WHC-EP-0625}

This page intentionally left blank. 


\section{Tank 241-TX-109}

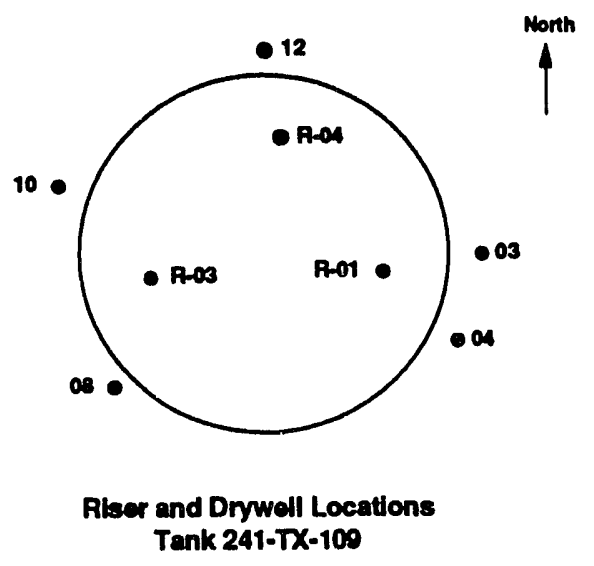

Number of External Drywells: 5

Number of Lateral Wells: None

\section{Tank Status}

Contents

Type: Non-Complexed Waste

Total Waste: $384 \mathrm{~K}$ gallons

Supernate Volume: OK gallons

Drainable Interstitial Liquid: $10 \mathrm{~K}$ gallons

Isolation Status

Date Interim Stabilized: 04/19/83

Date Interim Isolated: $08 / 84$

\section{Surface Level/Leak Status}

Integrity Category: Sound

FIC Surface Level: Intrusion level set at 148.60 Inches (12/28/92)

Surface Level Comments: Intrusion Mode

Last Photographed: 10/24/89

Photo Interpretation Comments:

Photographs show the FIC and manual tape plummet contacting solids. The FIC plummet appears to be directly over a hole in the salt cake. There is no visible liquid.

Temperature Status

Highest temperature during 1992:

\section{Drywell Status}

Comments: Current drywell profiles were stable and consistent with established baseline profiles.
Tank Description

Type: Single Shell

Constructed: 1948

In-service: 1950

Diameter: $\mathbf{7 5}^{\circ}$

Usable Depth: 23'

Capacity: $758 \mathrm{~K}$ gallons

Bottom shape: Dish

Hanford Coordinates:

$41,854^{\circ}$ North

75,750' West

Ventilation: Passive

\section{Leak Detection System}

Surface Level:

FIC Riser- R-04

Manual Tape Riser- R-01

LOW Riser(s)- R-03 
WHC-EP-0625

This page intentionally left blank. 


\section{Tank 241-TX-110}

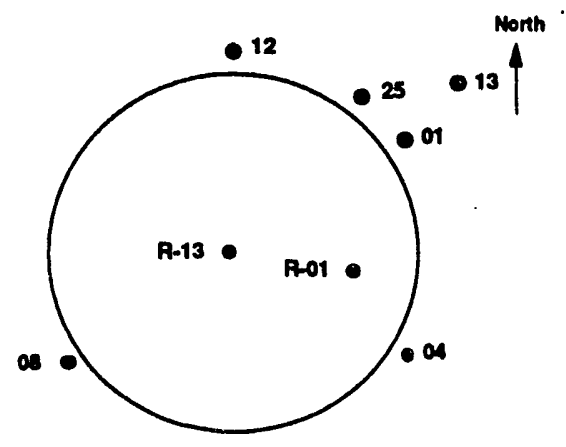

Riser and Dryweil Locations Tank 241-TX-110
Tank Description Type: Single Shell Constructed: 1948 In-service: 09/49 Diameter: $75^{\prime}$ Usable Depth: $23^{\circ}$ Capacity: $758 \mathrm{~K}$ gallons Bottom shape: Dish Hanford Coordinates: 41,854' North 75,852' West Ventilation: Passive

\section{Leak Detection System}

Surface Level:

FIC Riser- None

Manual Tape Riser- R-01

LOW Riser(s)- R-13

Number of External Drywells: 6

Number of Lateral Wells: None

\section{Tank Status}

Contents

Type: Non-Complexed Waste

Total Waste: $462 \mathrm{~K}$ gallons

Supernate Volume: OK gallons

Drainable Interstitial Liquid: $15 \mathrm{~K}$ gallons

Isolation Status

Date Interim Stabilized: 04/05/83

Date Interim Isolated: $08 / 84$

\section{Surface Level/Leak Status}

Integrity Category: Assumed Leaker

Date Declared Confirmed or Assumed Leaker: 1977

Leak Volume Estimate: $7.8 \mathrm{~K}$ gallons $=1 / 19$ th of $150 \mathrm{~K}$ gallons

Manual Tape Surface Level: 147.00 Inches (10/01/92)

Last Photographed: 10/24/89

Photo Interpretation Comments:

Photographs show an uneven surface of salt cake with no visible liquid. The manual tape was removed to obtain pictures, but would contact solids in a

\section{Temperature Status} depression with a discarded donoughnut and tape in the immediate area.

Highest recent temperature: $77^{\circ} \mathrm{F}(04 / 24 / 91)$

Comments: Temperatures are stable.

Drywell Status

Comments: Current drywell profiles were stable and consistent with established baseline profiles. 


\section{WHC-EP-0625}

This page intentionally left blank. 


\section{Tank 241-TX-111}

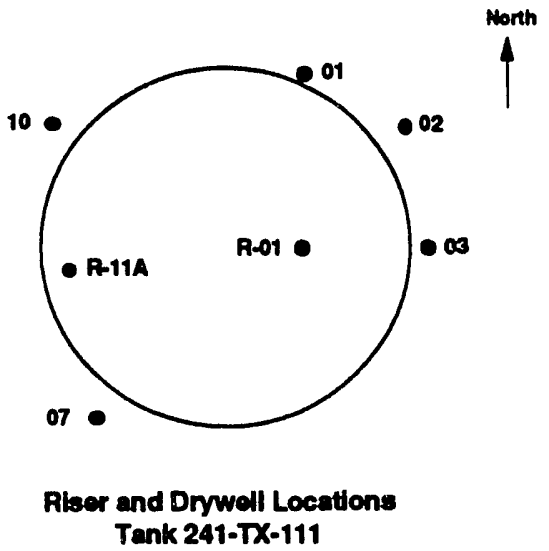

Tank 241-TX-111
Tank Description

Type: Single Shell

Constructed: 1948

In-service: $03 / 50$

Dlameter: $\mathbf{7 5}^{\circ}$

Usable Depth: 23'

Capactty: $758 \mathrm{~K}$ gallons

Bottom shape: Dish

Hanford Coordinates:

41,854' North

75,954' West

Ventilation: Passive

\section{Leak Detection System \\ Surface Level:}

FIC Riser- None

Manual Tape Riser- R-01

LOW Riser(s)- R-11A

Number of External Drywells: 5

Number of Lateral Wells: None

\section{Tank Status}

\section{Contents}

Type: Non-Complexed Waste

Total Waste: $370 \mathrm{~K}$ gallons

Supernate Volume: OK gallons

Drainable Interstitial Liquid: $9 \mathrm{~K}$ gallons

Isolation Status

Date Interim Stabilized: 04/05/83

Date Interim Isolated: $08 / 84$

\section{Surface Level/Leak Status}

Integrity Category: Sound

Manual Tape Surface Level: 137.00 Inches (10/01/92)

Last Photographed: 09/12/89

Photo Interpretation Comments:

Photographs show a dry, cracked, uneven surface of salt-cake with no visible liquid.

Temperature Status

Highest temperature during 1992:

\section{Drywell Status}

Comments: Current drywell profiles were stable and consistent with established baseline profiles. 
WHC-EP-0625

This page intentionally left blank. 


\section{Tank 241-TX-112}

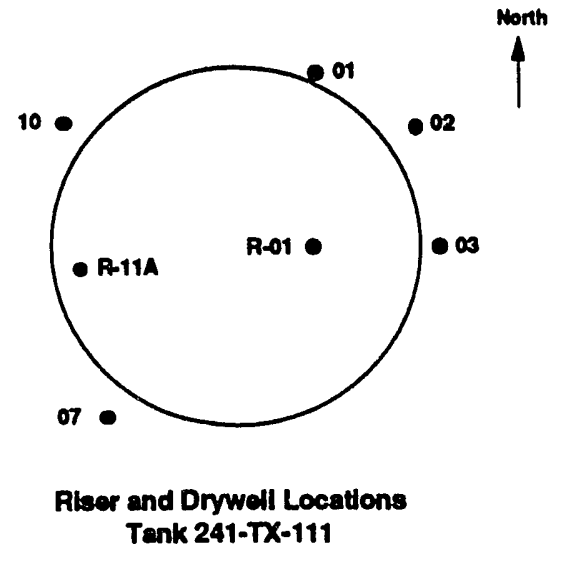

Number of External Drywells: 6 Number of Lateral Wells: None

\section{Tank Status}

Contents

Type: Non-Complexed Waste

Total Waste: $649 \mathrm{~K}$ gallons

Supernate Volume: OK gallons

Drainable Interstitial Liquid: $24 \mathrm{~K}$ gallons

Isolation Status

Date Interim Stabilized: 04/05/83

Date Interim Isolated: $08 / 84$

\section{Surface Level/Leak Status}

Integrity Category: Sound

Manual Tape Surface Level: 237.50 Inches (10/01/92)

Last Photographed: 11/19/87

Photo Interpretation Comments:

Photographs show a dry, uneven surface of salt-cake with no visible liquid. The manual tape donoughnut plummet is contacting solids in a small depression.

\section{Temperature Status}

Highest temperature during 1992:

Drywell Status

Comments: Current drywell profiles were stable and consistent with established baseline profiles. 
WHC-EP-0625

This page intentionally left blank. 


\section{Tank 241-TX-113}

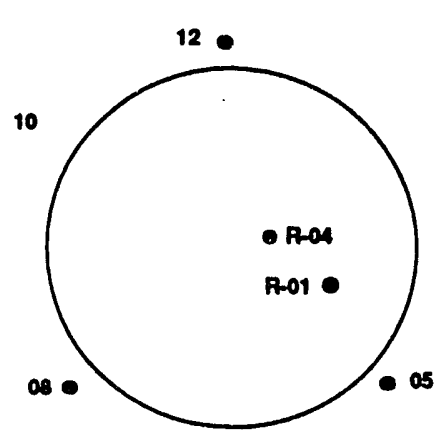

Rlaer and Drywoll Locations Tank 241-TX-113

Number of External Drywells: 3 Number of Lateral Wells: None

\section{Tank Status}

\section{Contents}

Type: Non-Complexed Waste Total Waste: $607 \mathrm{~K}$ gallons Supernate Volume: OK gallons

Drainable Interstitial Liquid: $16 \mathrm{~K}$ gallons Isolation Status

Date Interim Stabilized: 04/05/83

Date Interim Isolated: $08 / 84$

\section{Surface Level/Leak Status}

Integrity Category: Assumed Leaker

Date Declared Confirmed or Assumed Leaker: 1974

Leak Volume Estimate: $7.8 \mathrm{~K}$ gallons $=1 / 19$ th of $150 \mathrm{~K}$ gallons

Manual Tape Surface Level: 215.50 Inches (10/01/92)

Last Photographed: 04/11/83

Photo Interpretation Comments:

Photographs show an uneven surface of salt-cake and no visible liquid. The

\section{Temperature Status} manual tape pencil plummet is contacting solids in a hole.

Highest temperature during 1992:

\section{Drywell Status}

Comments: Current drywell profiles were stable and consistent with established baseline profiles. 


\section{WHC-EP-0625}

This page intentionally left blank. 


\section{Tank 241-TX-114}

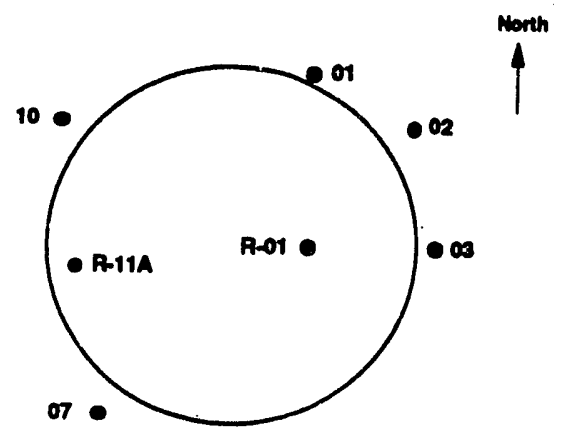

Alect and Drywell Locations Tank 241-TX-111

Number of External Drywells: 3

Number of Lateral Wells: None

\section{Tank Status}

\section{Contents}

Type: Non-Complexed Waste

Total Waste: $535 \mathrm{~K}$ gallons

Supernate Volume: OK gallons

Drainable Interstitial Liquid: $15 \mathrm{~K}$ gallons

Isolation Status

Date Interim Stabllized: 04/05/83

Date Partially Interim Isolated: 12/15/82

Date Interim Isolated: 08/84

\section{Surface Level/Leak Status}

Integrity Category: Assumed Leaker

Date Declared Confirmed or Assumed Leaker: 1974

Leak Volume Estimate: $7.8 \mathrm{~K}$ gallons $=1 / 19$ th of $150 \mathrm{~K}$ gallons

Manual Tape Surface Level: 201.25 Inches (10/01/92)

Last Photographed: 04/11/83

Photo Interpretation Comments:

Photographs from 09/22/80 continue to show a larger liquid pool at the salt well than $05 / 28 / 80$ and $10 / 07 / 77$ photographs. Photographs from $04 / 11 / 83$ show a
dry. uneven surface of salt-cake.

Temperature Status

Highest temp $\rightarrow$ rature during 1992:

Drywell Status

Comments: Current drywell proflles were stable and consistent with established baseline profiles.
Tank Description

Type: Single Shell

Constructed: 1948

-service: 04/51

Usable Depth: 23'

Capacity: 758K gallons

shape: Dish

71,852' West

Ventilation: Passive

\section{Leak Detection System}

FIC Riser- None

Manual Tape Riser- R-08

LOW Riser(s)- R-12A, R-09A, R-07 
WHC-EP-0625

This page intentionally left blank. 


\section{Tank 241-TX-115}

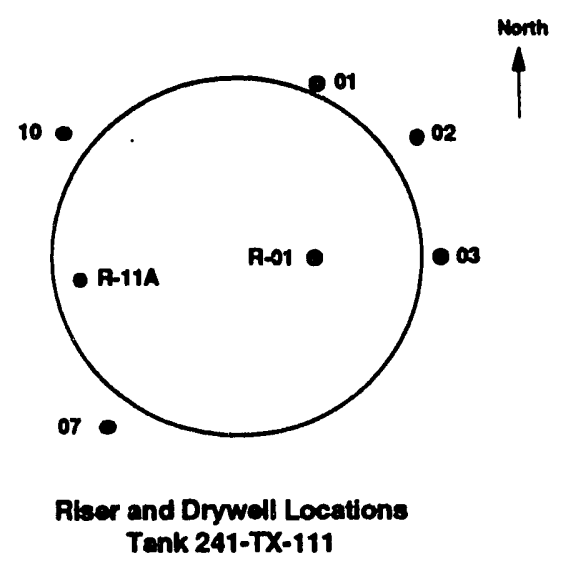

Number of External Drywells: 4

Number of Lateral Wells: None

\section{Tank Status}

Contents

Type: Non-Complexed Waste

Total Waste: $640 \mathrm{~K}$ gallons

Supernate Volume: OK gallons

Drainable Interstitial Liquid: $19 \mathrm{~K}$ gallons

Isolation Status

Date Interim Stabilized: 09/29/83

Date Partially Interim Isolated: $02 / 03 / 83$

Date Interim Isolated: $08 / 84$

\section{Surface Level/Leak Status}

Integrity Category: Assumed Leaker

Date Declared Confirmed or Assumed Leaker: 1977

Leak Volume Estimate: $7.8 \mathrm{~K}$ gallons $=1 / 19$ th of $150 \mathrm{~K}$ gallons

Manual Tape Surface Level: 201.50 Inches (10/01/92)

Last Photographed: 06/15/88

Photo Interpretation Comments:

Photographs from $01 / 28 / 81$ show the manual tape plummet contacting solids and a larger pool of liquid at the salt well than previous photos. Photographs from $03 / 28 / 83$ show no visible liquid. The manual tape plummet is contacting salt

Temperature Status cake near the edge of a depression caused by slumpage from salt well pumping.

Highest temperature during 1992:

Drywell Status

Comments: Current drywell profiles were stable and consistent with established baseline profiles. 


\section{WHC-EP-0625}

This page intentionally left blank. 


\section{Tank 241-ҮX-116}

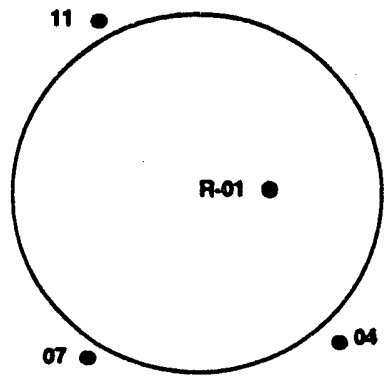

Riser and Drywell Locations Tank 241-TX-116

Number of External Drywells: 3

Number of Lateral Wells: None

\section{Tank Status}

Contents

Type: Non-Complexed Waste

Total Waste: $631 \mathrm{~K}$ gallons

Supernate Volume: OK gallons

Drainable Interstitial Liquid: 23K gallons

Isolation Status

Date Interim Stabilized: 04/05/83

Date Interim Isolated: $08 / 84$

Surface Level/Leak Status

Integrity Category: Assumed Leaker

Date Declared Confirmed or Assumed Leaker: 1977

Leak Volume Estimate: $7.8 \mathrm{~K}$ gallons $=1 / 19$ th of $150 \mathrm{~K}$ gallons

Manual Tape Surface Level: 212.25 Inches (10/01/92)

Last Photographed: 10/17/89

Photo Interpretation Comments:

Photographs from $01 / 28 / 81$ and $04 / 11 / 83$ reveal a dry, cracked surface and no

Temperature Status visible liquid.

Highest temperature during 1992:

\section{Drywell Status}

Comments: Current drywell profiles were stable and consistent with established baseline profiles. 
WHC-EP-0625

This page intentionally left blank. 


\section{Tank 241-TX-117}

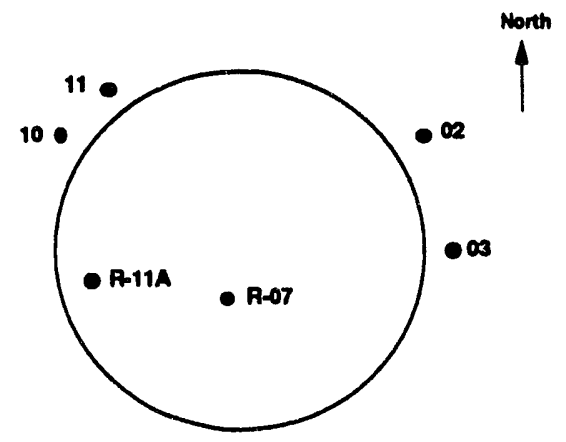

Riser and Drywell Locations Tank 241-TX-117

Number of External Drywells: 4

Number of Lateral Wells: None

\section{Tank Status}

Contents

Type: Non-Complexed Waste

Total Waste: $626 \mathrm{~K}$ gallons

Supernate Volume: OK gallons

Drainable Interstitial Liquid: $8 \mathrm{~K}$ gallons

Isolation Status

Date Interim Stabllized: $03 / 28 / 83$

Date Interim Isolated: $08 / 84$

Surface Level/Leak Status

Integrity Category: Assumed Leaker

Date Declared Confirmed or Assumed Leaker: 1977

Leak Volume Estimate: $7.8 \mathrm{~K}$ gallons $=1 / 19$ th of $150 \mathrm{~K}$ gallons

Manual Tape Surface Level: 183.00 Inches (10/01/92)

Last Photographed: 04/11/83

Photo Interpretation Comments:

Photographs show mounds of diatomaceous earth with no visible liquid. The

manual tape plummet is over a deep hole where a pump has been removed.

Temperature Status

Highest temperature during 1992:

\section{Drywell Status}

Comments: Current drywell profiles were stable and consistent with established baseline profiles. 
WHC-EP-0625

This page intentionally left blank. 


\section{Tank 241-TX-118}

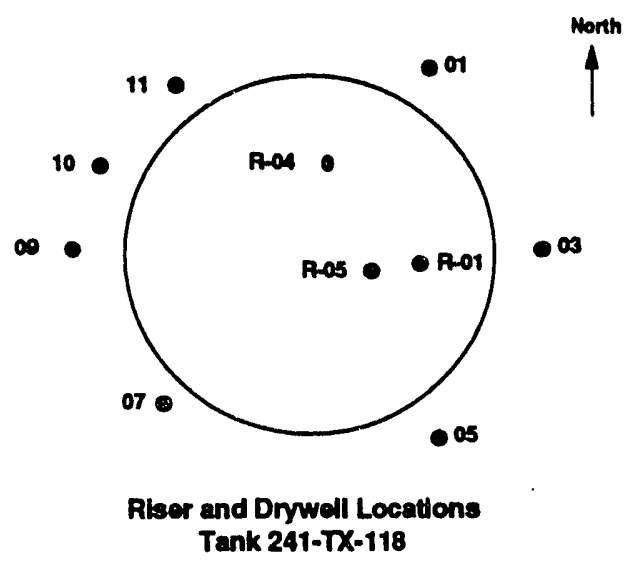

Number of External Drywells: 7

Number of Lateral Wells: None

\section{Tank Status}

Watch List: Ferrocyanide, Organic Salts

\section{Contents}

Type: Non-Complexed Waste

Total Waste: $347 \mathrm{~K}$ gallons

Supernate Volume: OK gallons

Drainable Interstitial Liquid: $27 \mathrm{~K}$ gallons

Isolation Status

Date Interim Stabilized: 04/05/83

Date Interim Isolated: $08 / 84$

Surface Level/Leak Status

Integrity Category: Sound

FIC Surface Level: Intrusion level set at 117.70 Inches (12/14/92)

Surface Level Comments: Intrusion Mode

Last Photographed: 12/19/79

Photo Interpretation Comments:

There are no current photographs because of jet salt well pumping.

Temperature Status

Highest temperature during 1992: $78.4{ }^{\circ} \mathrm{F}$ (12/20/92)

Comments: Temperatures are stable.

\section{Drywell Status}

Comments: Current drywell profiles were stable and consistent with established baseline profiles.
Tank Description

Type: Single Shell

Constructed: 1948

In-service: $04 / 51$

Dlameter: $7^{\circ}$

Usable Depth: 23'

Capacity: $758 \mathrm{~K}$ gallons

Bottom shape: Dish

Hanford Coordinates:

42,058' North

75,954' West

Ventilation: Passive

\section{Leak Detection System \\ Surface Level:}

FIC Riser- R-04

Manual Tape Riser- R-01

LOW Riser(s)- R-05 
WHC-EP-0625

This page intentionally left blank. 


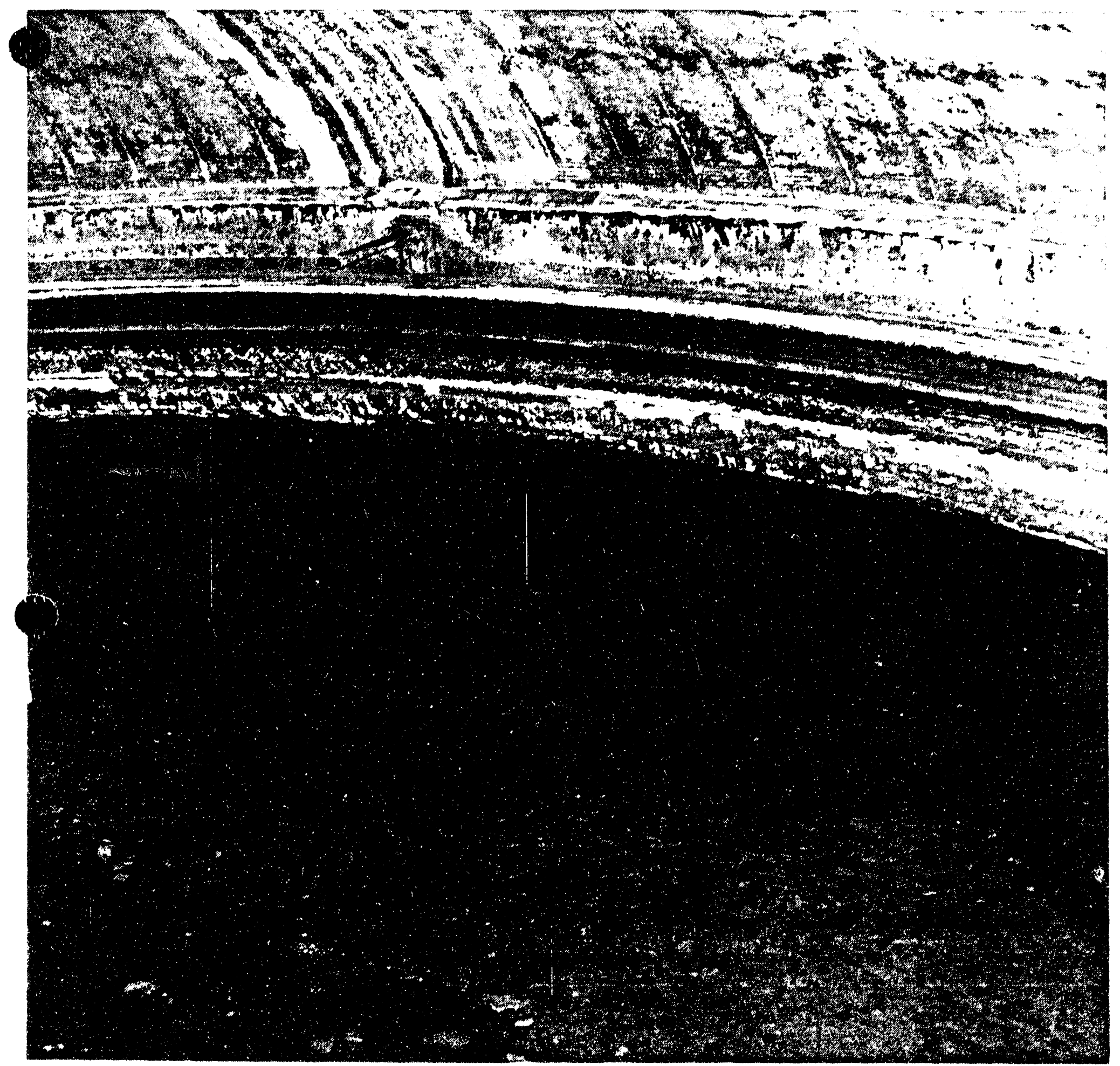


WHC-EP-0625

This page intentionally left blank. 


\section{Tank 241-TY-101}

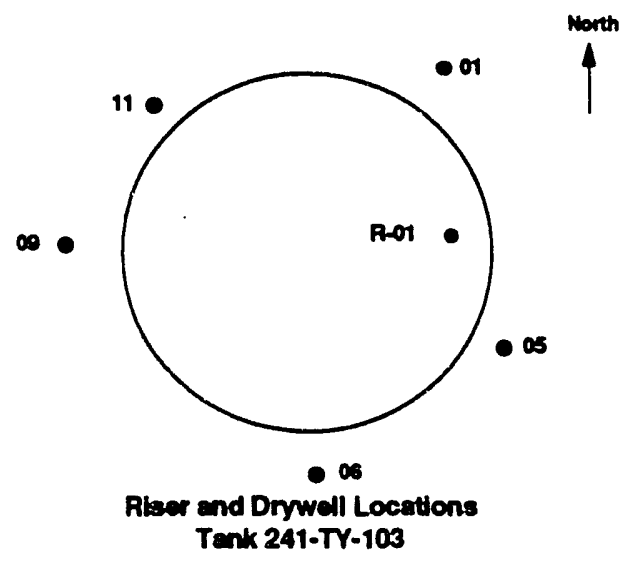

Number of External Drywells: 3

Number of Lateral Wells: None

\section{Tank Status}

Watch List: Ferrocyanide

Contents

Type: Non-Complexed Waste

Total Waste: $118 \mathrm{~K}$ gallons

Supermate Volume: OK gallons

Drainable Interstitial Liquid: OK gallons

Isolation Status

Date Interim Stabilized: 04/05/83

Date Partially Interim Isolated: $12 / 15 / 82$

Date Interim Isolated: $10 / 30 / 84$

\section{Surface Level/Leak Status}

Integrity Category: Assumed Leaker

Date Declared Confirmed or Assumed Leaker: 1973

Leak Volume Estimate: < 1,000 Gallons (1980)

FIC Surface Level: Intrusion level set at 51.40 Inches (12/14/92)

Surface Level Comments: Intrusion Mode

Last Photographed: 08/22/89

Photo Interpretation Comments:

Photographs show a dry, caked, uneven surface with no visible liquid.

\section{Temperature Status}

Highest temperature during 1992: $77^{\circ} \mathrm{F}(09 / 30 / 92)$

Comments: Temperatures are stable.

Drywell Status

Comments: Current drywell profiles were stable and consistent with established baseline profiles.
Tank Description

Type: Single Shell

Constructed: 1952

In-service: 1953

Out of Service: 1973

Diameter: $75^{\circ}$

Usable Depth: 23'

Capacity: 758K gallons

Bottom shape: Dish

Hanford Coordinates:

42,602' North

75.852' West

Ventilation: Passive

\section{Leak Detection System \\ Surface Level: \\ FIC Riser- R-01 \\ Manual Tape Riser- None \\ LOW Riser(s)- None}


WHC-EP-0625

This page intentionally ieft blank. 
WHC-EP-0625

\section{Tank: 241-TY-101}

\section{August 1989}

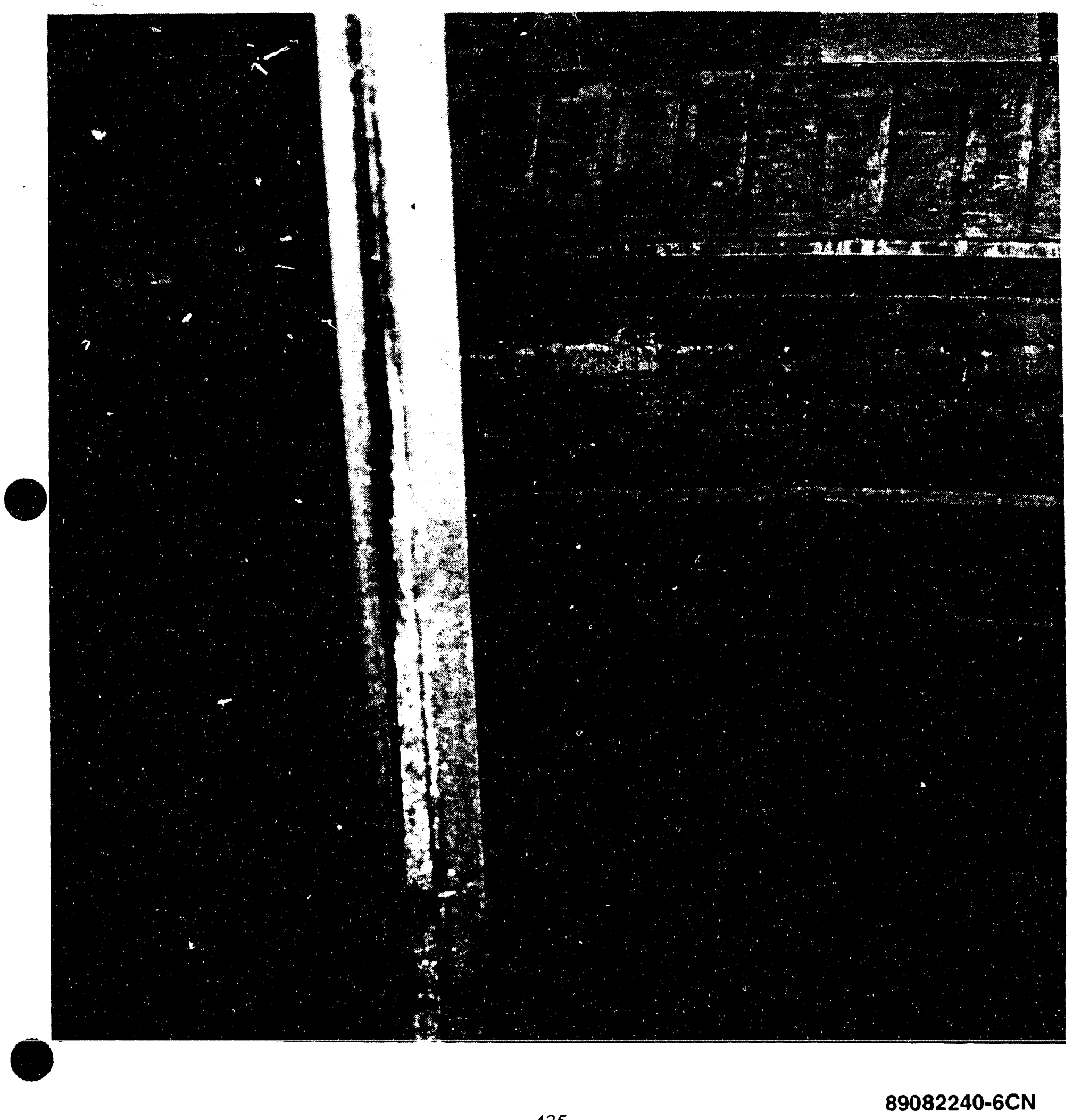


WHC-EP-0625

This page intentionally left blank. 


\section{Tank 241-TY-102}

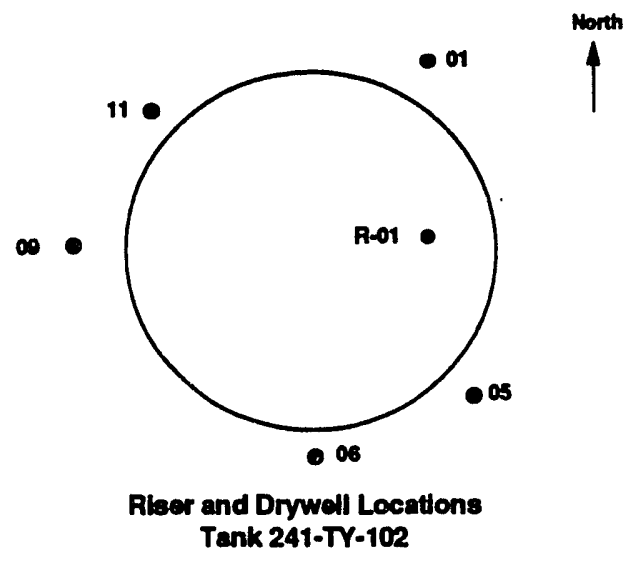

Number of External Drywells: 5

Number of Lateral Wells: None

\section{Tank Status}

\section{Contents}

Type: Non-Complexed Waste

Total Waste: $64 \mathrm{~K}$ gallons

Supernate Volume: OK gallons

Drainable Interstitial Liquid: $14 \mathrm{~K}$ gallons

\section{Isolation Status}

Date Interim Stabilized: 04/02/84

Date Interim Isolated: $12 / 15 / 82$

\section{Surface Level/Leak Status}

Integrity Category: Sound

FIC Surface Level: 31.70 Inches (12/21/92)

Last Photographed: 07/07/87

Photo Interpretation Comments:

Photographs show the tank contains mostly solids with a shallow pool of liquid in the center of the tank. Photo comparisons show more surface liquid and the small puddle beneath the FIC plummet engulfed by the larger pool of liquild.

Temperature Status

Highest temperature during 1992: $69^{\circ} \mathrm{F}(11 / 05 / 92)$

Comments: Temperatures are stable.

Drywell Status

Comments: Current drywell profiles were stable and consistent with established baseline profiles. 
WHC-EP-0625

This page intentionally left blank. 


\section{Tank 241-TY-103}

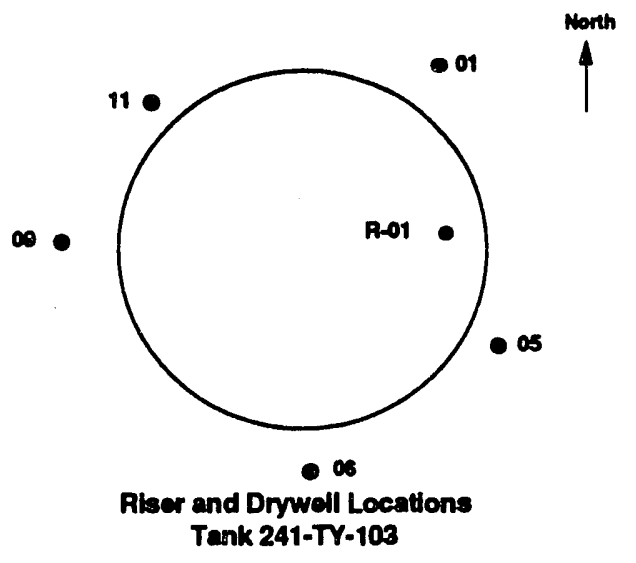

Number of External Drywells: 3

Number of Lateral Wells: None

\section{Tank Status}

Watch List: Ferrocyanide

Contents

Type: Non-Complexed Waste

Total Waste: $162 \mathrm{~K}$ gallons

Supernate Volume: OK gallons

Drainable Interstitial Liquid: $5 \mathrm{~K}$ gallons

Isolation Status

Date Interim Stabilized: 03/01/83

Date Partially Interim Isolated: $12 / 15 / 82$

Date Interim Isolated: $10 / 30 / 84$

\section{Surface Level/Leak Status}

Integrity Category: Assumed Leaker

Date Declared Confirmed or Assumed Leaker: 1973

Leak Volume Estimate: 3,000 Gallons (1986)

FIC Surface Level: Intrusion level set at 67.50 Inches (12/28/92)

Surface Level Comments: Intrusion Mode

Last Photographed: 08/22/89

Photo Interpretation Comments:

Photographs show an uneven surface of solids and a small pool of liquild around the salt well. The FIC plummet is contacting solids.

\section{Temperature Status}

Highest temperature during 1992: $79.2^{\circ} \mathrm{F}(11 / 19 / 92)$

\section{Drywell Status}

Comments: Temperatures are stable.

Comments: Current drywell profles were stable and consistent with established baseline profiles.
Tank Description

Type: Single Shell

Constructed: 1952

n-service: $07 / 16 / 53$

Bottom shape: Dish

75,852' West

Ventilation: Passive

\section{Leak Detection System}

Surface Level:

FIC Riser- R-01

Manual Tape Riser- None

LOW Riser(s)- R-03 
WHC-EP-0625

This page intentionally left blank. 
WHC HPOADS

\section{Tank: 241-TY-103}

\section{August 1989}

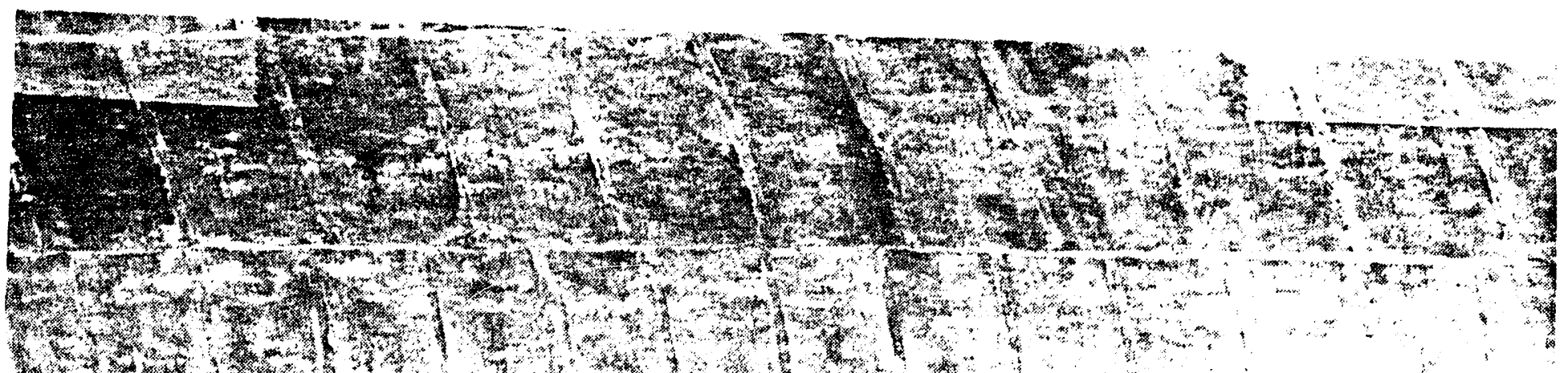

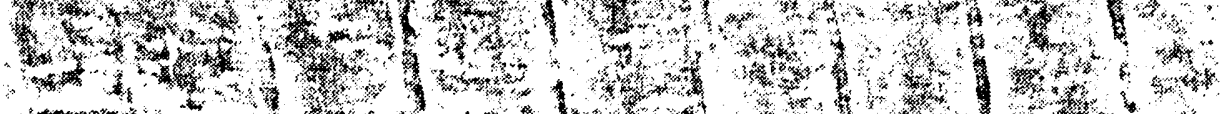
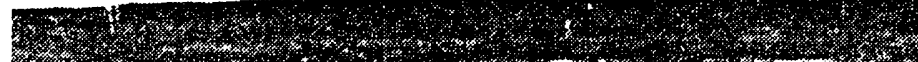

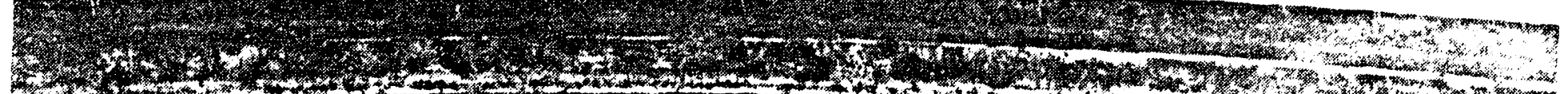

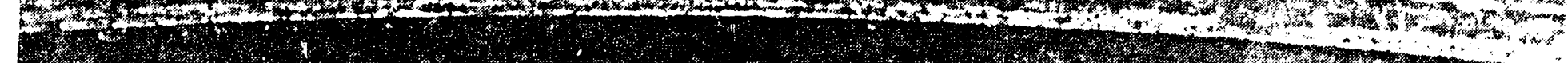
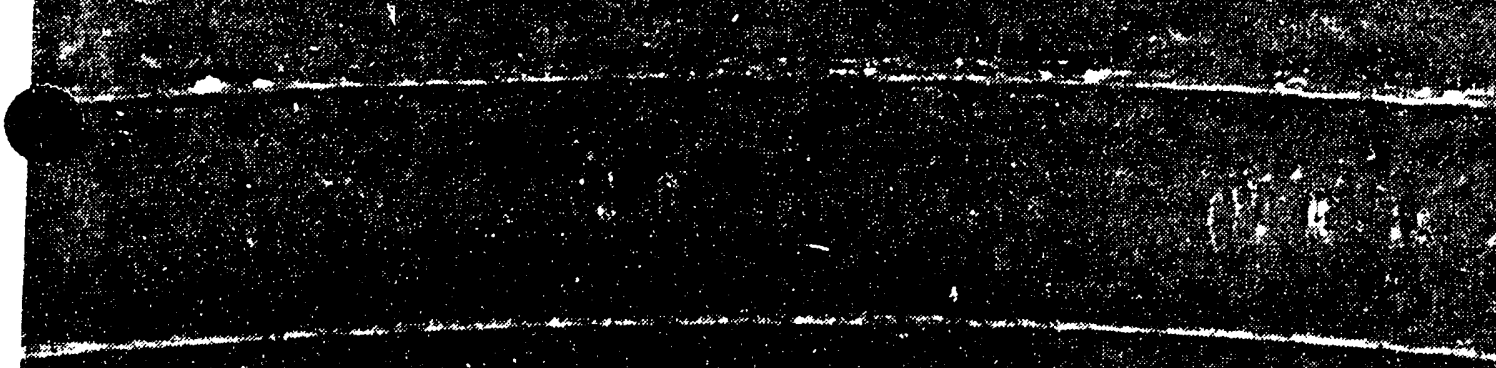

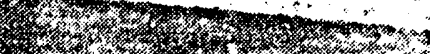

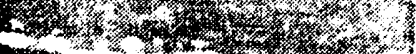
1

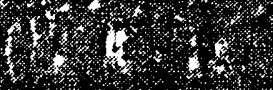

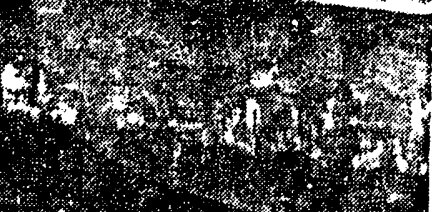
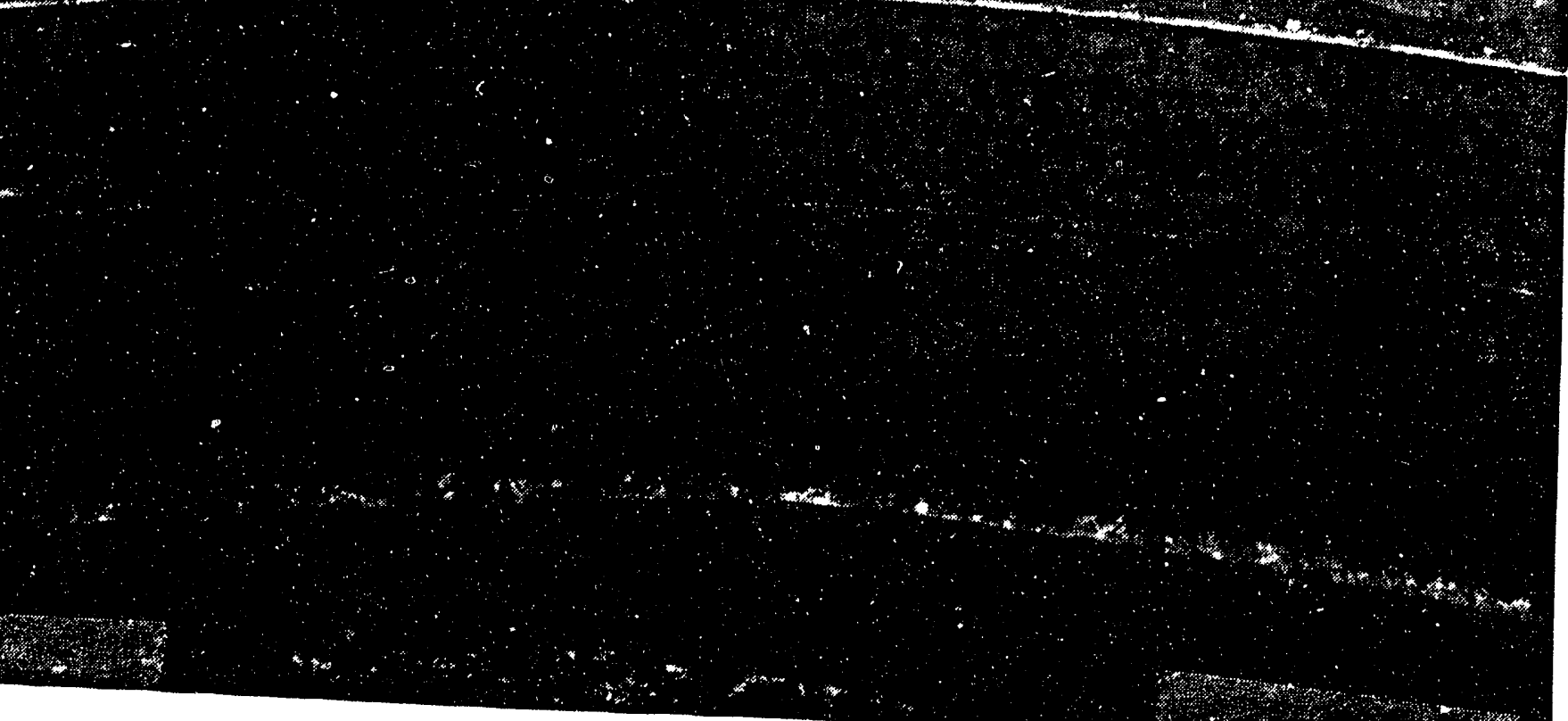
WHC-FP-0625

This page intentionally left blank. 


\section{Tank 241-TY-104}

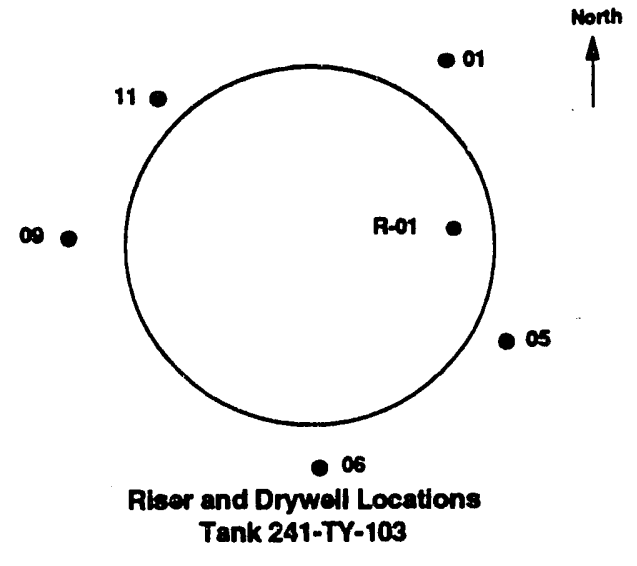

Number of External Drywells: 5

Number of Lateral Wells: None

\section{Tank Status}

Watch List: Ferrocyanide

Contents

Type: Non-Complexed Waste

Total Waste: $46 \mathrm{~K}$ gallons

Supernate Volume: $3 \mathrm{~K}$ gallons

Drainable Interstitial Liquid: $12 \mathrm{~K}$ gallons

Isolation Status

Date Interim Stabilized: $11 / 01 / 83$

Date Interim Isolated: $12 / 15 / 82$

Surface Level/Leak Status

Integrity Category: Assumed Leaker

Date Declared Confirmed or Assumed Leaker: 1981

Leak Volume Estimate: 1,400 Gallons (1986)

FIC Surface Level: 23.70 Inches $(11 / 27 / 92)$

Last Photographed: 11/03/87

Photo Interpretation Comments:

Photographs from $01 / 31 / 80$ show exposed solids along the outer edge of the tank and a much larger pool of liquid toward the center of the tank than 175 photographs. The FIC plummet is contacting liquid. 04/05/83 photos appear unchanged.

\section{Temperature Status}

Highest temperature during 1992: $78^{\circ} \mathrm{F}(09 / 30 / 92)$

Comments: Temperatures are stable.

\section{Drywell Status}

Comments: Current drywell profiles were stable and consistent with established

baseline profiles.
Tank Description

Type: Single Shell

Constructed: 1952

In-service: $08 / 10 / 53$

Out of Service: $03 / 74$

Diameter: $75^{\circ}$

Usable Depth: 23'

Capacity: $758 \mathrm{~K}$ gallons

Bottom shape: Dish

Hanford Coordinates:

75,954' West

Ventilation: Passive

\section{Leak Detection System}

Surface Level:

FIC Riser- R-01

Manual Tape Riser- None

LOW Riser(s)- None
42,500' North 
WHC-EP-0625

This page intentionally left blank. 
WHC-EP-0625

\title{
Tank: 241-TY-104
}

\section{November 1987}

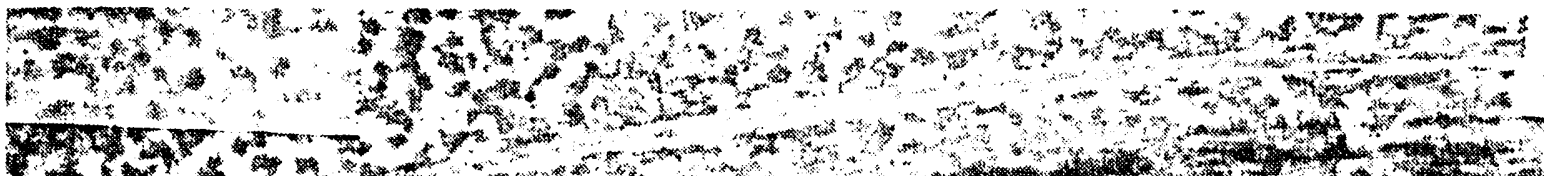
1.

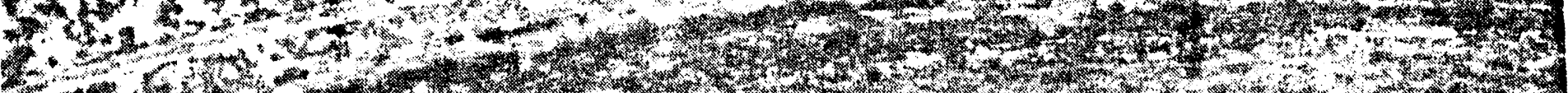

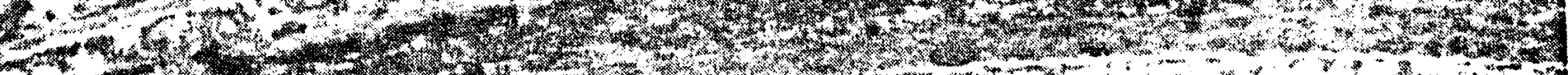

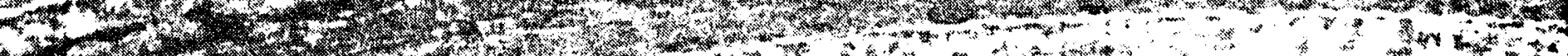

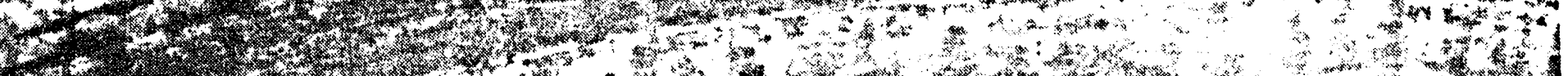

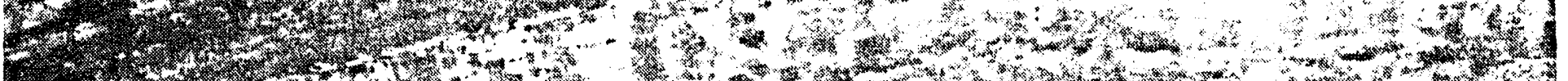

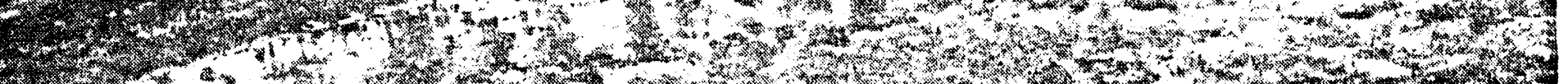

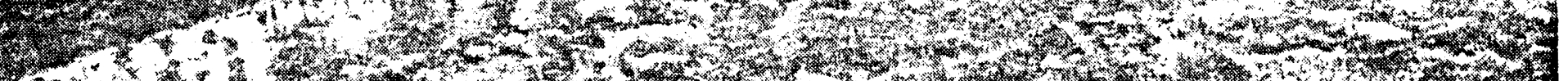

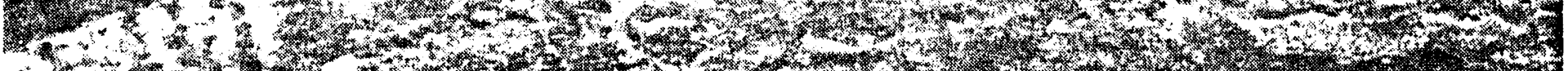

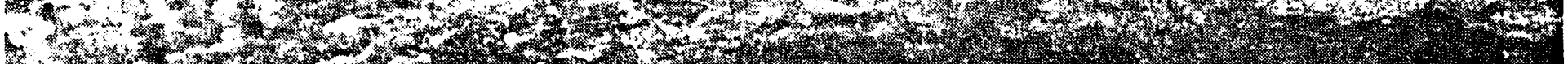

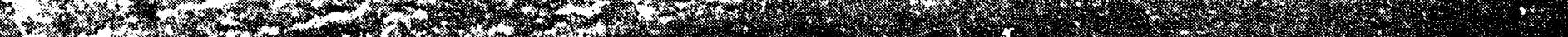

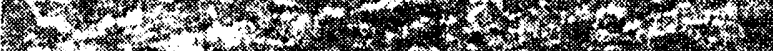

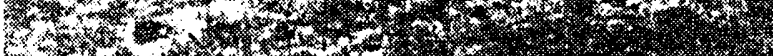

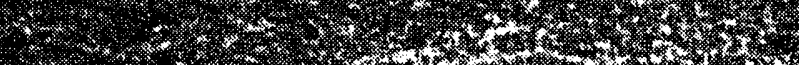

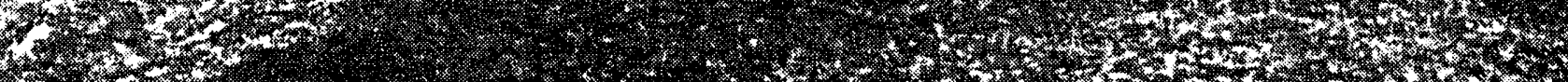

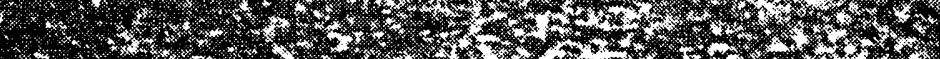
J6

6

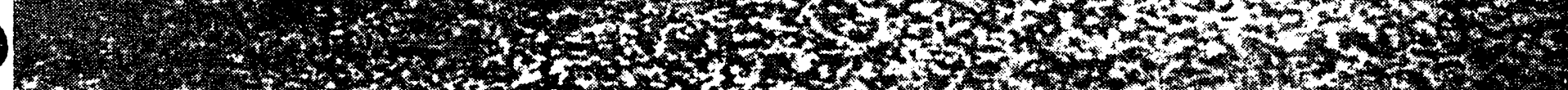

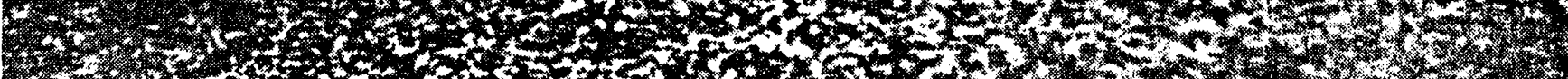

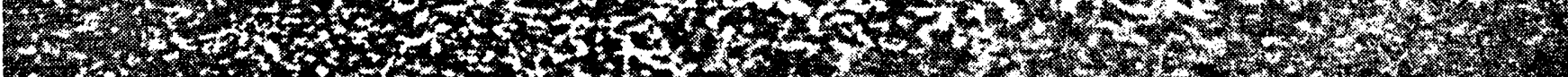

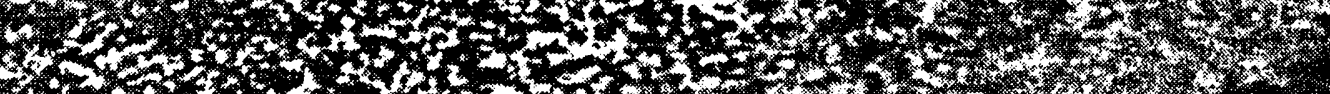

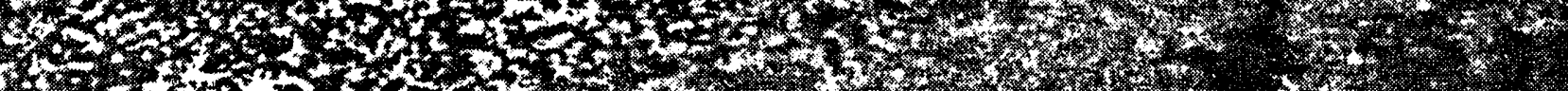

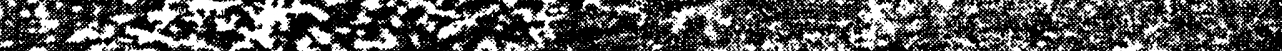

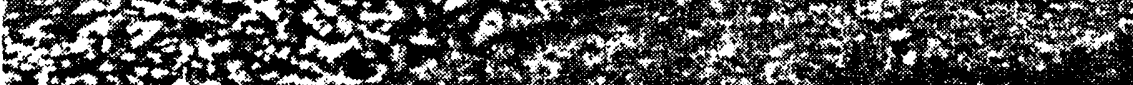

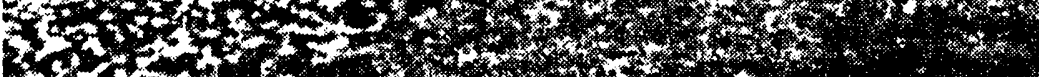

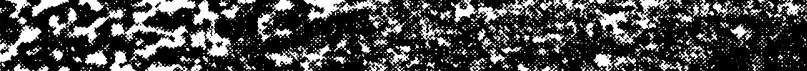

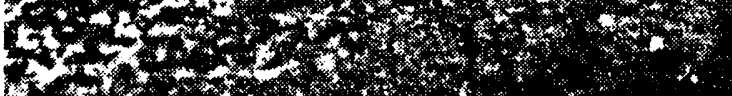

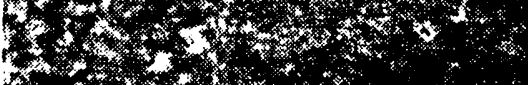

(n)

$+x_{\infty}^{4}$ 
WHC-EP-0625

This page intentionally left blank. 


\section{Tank 241-TY-105}

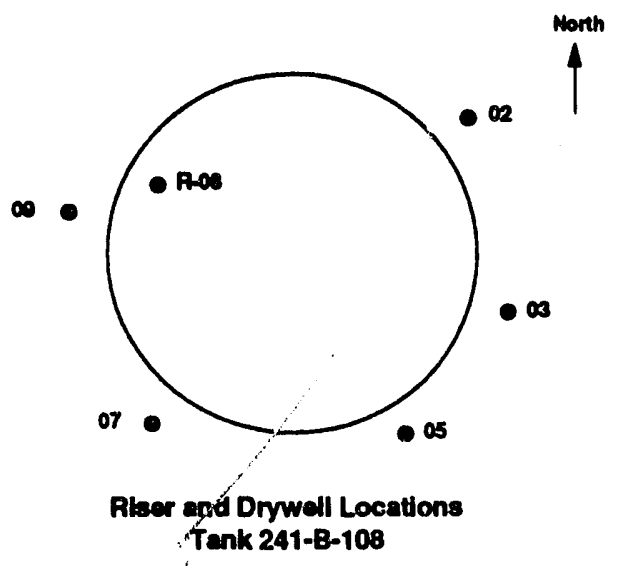

Number of External Drywells: 1

Number of Lateral Wells: None

Tank Status

Contents

Type: Non-Complexed Waste

Total Waste: $231 \mathrm{~K}$ gallons

Supernate Volume: OK gallons

Drainable Interstitial Liquid: OK gallons

Isolation Status

Date Interim Stabilized: 03/01/83

Date Partially Interim Isolated: $12 / 15 / 82$

Date Interim Isolated: 10/30/84

\section{Surface Level/Leak Status}

Integrity Category: Assumed Leaker

Date Declared Confirmed or Assumed Leaker: 1960

Leak Volume Estimate: 35,000 Gallons (1986).

Manual Tape Surface Level: 86.50 Inches (10/01/92)

Last Photographed: 09/07/89

Photo Interpretation Comments:

Photographs show the manual tape plummet contacting what appear to be soft solids and a pool of liquid around the salt well that was not visible in the $10 / 14 / 82$ photographs.

\section{Temperature Status}

Highest temperature during 1992: $81.40^{\circ} \mathrm{F}(01 / 12 / 92)$

Comments: Temperatures are stable.

\section{Drywell Status}

Comments: Current drywell profiles were stable and consistent with established baseline proflles.
Tank Description

Type: Single Shell

Constructed: 1952

In-service: $01 / 29 / 53$

of Service: $09 / 60$

Capacity: $758 \mathrm{~K}$ gallons

Bottom shape: Dish

Hanford Coordinates:

75,852' West

Ventilation: Passive

\section{Leak Detection System}

Surface Level:

FIC Riser- None

Manual Tape Riser- R-02

LOW Riser(s)- None $42,400^{\prime}$ North 
WHC-EP-0625

This page intentionally left blank. 


\section{Tank 241-TY-106}

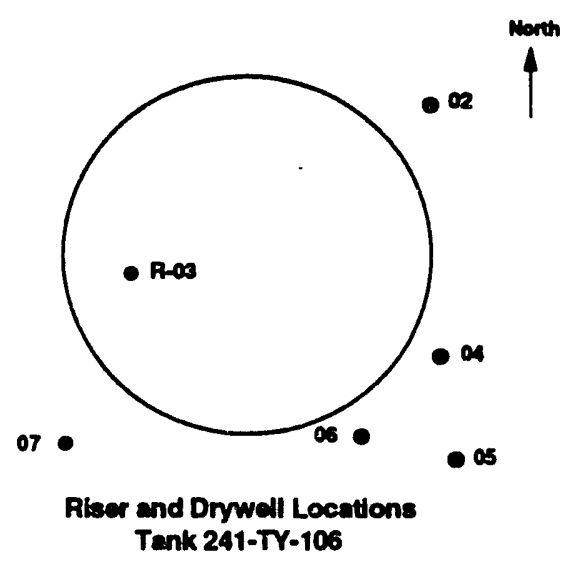

Number of External Drywells: 5

Number of Lateral Wells: None

\section{Tank Status}

\section{Contents}

Type: Non-Complexed Waste

Total Waste: $17 \mathrm{~K}$ gallons

Supernate Volume: OK gallons

Drainable Interstitial Liquid: OK gallons

\section{Isolation Status}

Date Interim Stabilized: $11 / 30 / 78$

Date Interim Isolated: 12/15/82

\section{Surface Level/Leak Status}

Integrity Category: Assumed Leaker

Date Declared Confirmed or Assumed Leaker: 1959

Leak Volume Estimate: 20.000 Gallons (1986)

Manual Tape Surface Level: 13.50 inches (10/01/92)

Last Photogiaphed: $08 / 22 ; 89$

Photo Interpretation Comments:

Photographs show a dry, cracked surface of sludge and no visible liquid.

\section{Temperature Status}

Highest temperature during 1992: $64.5^{\circ} \mathrm{F}(01 / 03 / 92)$

Comments: Temperatures are stable.

\section{Drywell Status}

Comments: Cirrent drywell profles were stable and consistent with established baseline profiles. 
WHC-EP-0625

This page intentionally left blank. 


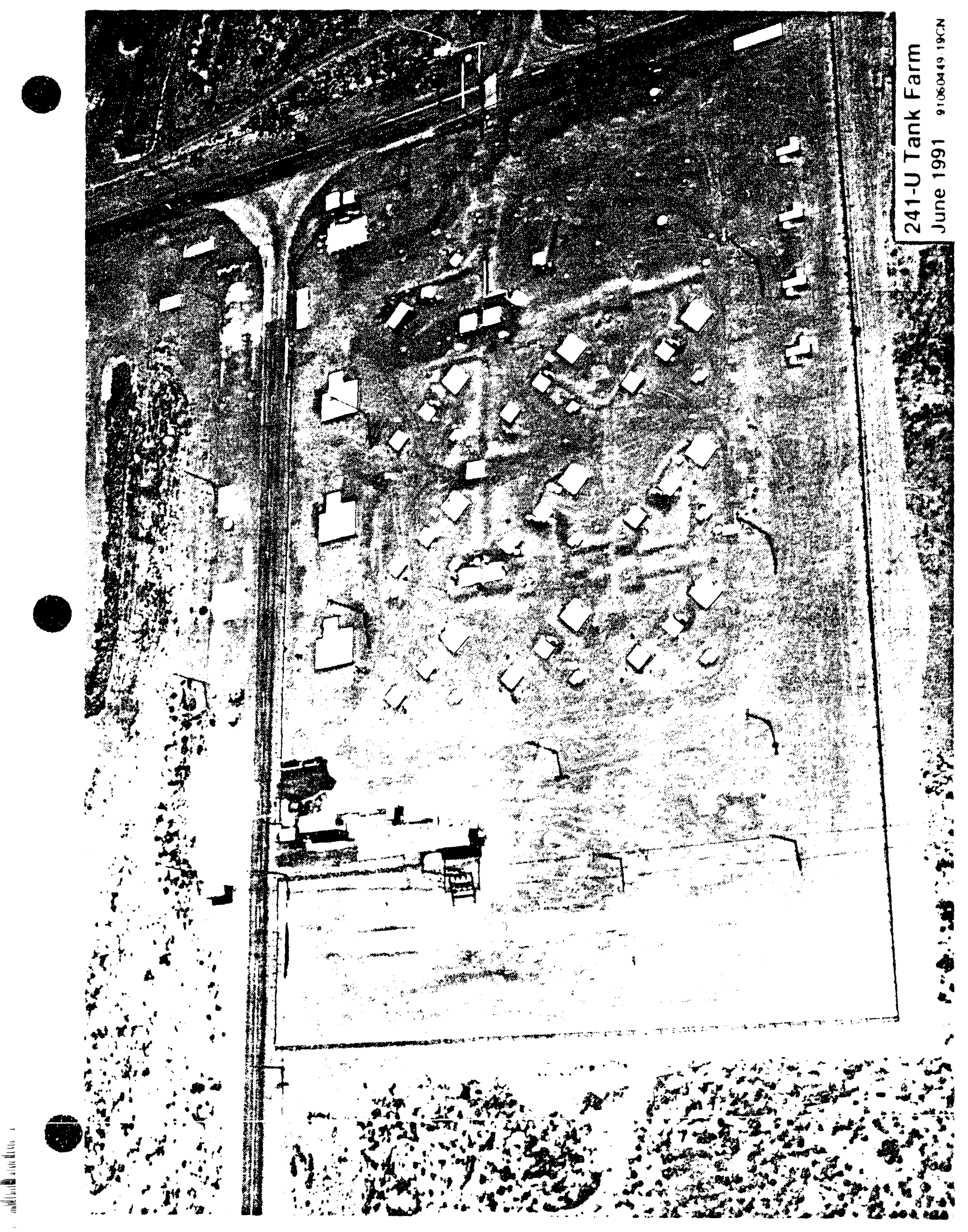


WHC-EP-0625

This page intentionally left blank. 


\section{Tank 241-U-101}

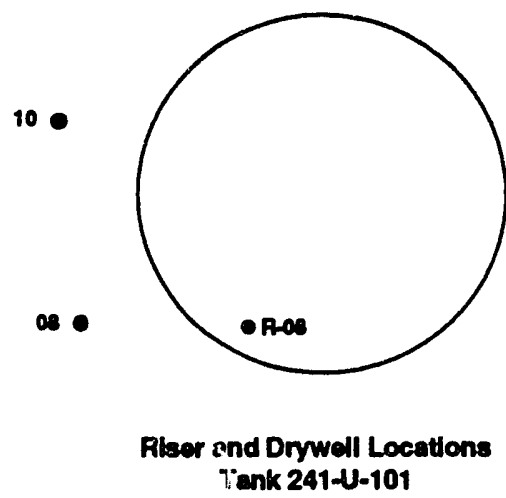

Number of External Drywells: 2 Number of Lateral Wells: None
Tank Description Type: Single Shell Constructed: 1944 In-service: $02 / 25 / 46$

Out of Service: $11 / 59$

Diameter: $75^{\circ}$

Usable Depth: $16^{\circ}$ Capacity: $530 \mathrm{~K}$ gallons Bottom shape: Dish Hanford Coordinates: $38,197^{\prime}$ North 75,637' West

Ventilation: Passive

\section{Leak Detection System}

Surface Level:

FIC Riser- None

Manual Tape Riser- R-08

LOW Riser(s)- None

\section{Tank Staius \\ Contents}

Type: Non-Complexed Waste

Total Waste: $25 \mathrm{~K}$ gallons

Supernate Volume: 3K gallons

Drainable Interstitial Liquid: OK gallons

\section{Isolation Status}

Date Interim Stabilized: $09 / 17 / 79$

Date Interim Isolated: $09 / 82$

\section{Surface Level/Leak Status}

Integrity Category: Assumed Leaker

Date Declared Confirmed or Assumed Leaker: 1959

Leak Volume Estimate: 30.000 Gallons (1986)

Manual Tape Surface Level: 3.75 Inches (10/01/92)

Last Photographed: 06/19/79

Photo Interpretation Comments:

Photographs are not clear (because of haze and radiation film damage) but do show a shallow, clear liquid surface and exposed solids around the perimeter of

\section{Temperature Status} the tank. The manual tape plummet is not clearly visible.

Highest temperature during 1992: $67.5^{\circ} \mathrm{F}(07 / 04 / 92)$

Comments: Temperatures are stable.

\section{Drywell Status}

Comments: Current drywell profiles were stable and consistent with established baseline profules. 


\section{WHC-EP-0625}

This page intentionally left blank. 


\section{Tank 241-U-102}

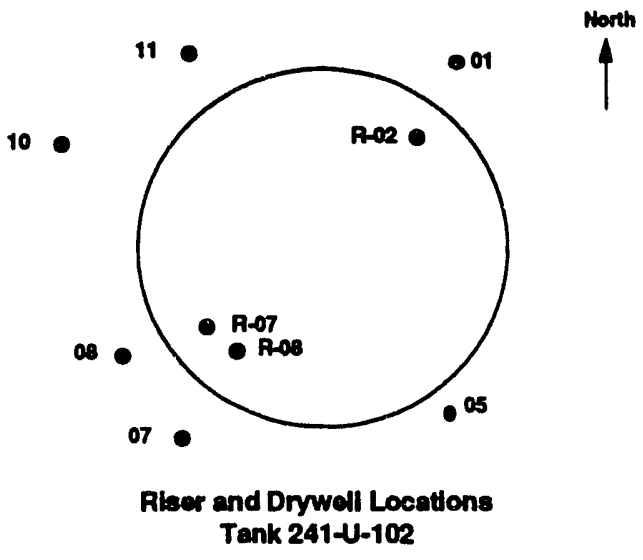

Number of External Drywells: 6

Number of Lateral Wells: None

Tank Status

Contents

Type: Non-Complexed Waste

Total Waste: $374 \mathrm{~K}$ gallons

Supernate Volume: $18 \mathrm{~K}$ gallons

Drainable Interstitial Liquid: $126 \mathrm{~K}$ gallons

Isolation Status

Date Partially Interim Isolated: $12 / 15 / 82$

Surface Level/Leak Status

Integnty Category: Sound

FIC Surface Level: Intrusion le'rel set at 131.60 Inches $(12 / 28 / 92)$

Surface Level Comments: Intrusion Mode

Last Photographed: 06/08/89

Photo Interpretation Comments:

Photograph comparisons from $07 / 26 / 83$ and 10/02/79 tend to show more surface liquid and less exposed salt cake around the tank perimeter. Pleces of salt cake have fallen away from the liner and the FIC plummet appears to be contacting exposed solids.

\section{Temperature Status}

Highest temperature during 1992: $152.6{ }^{\circ} \mathrm{F}(10 / 19 / 92)$

Comments: Temperatures are stable.

\section{Drywell Status}

Comments: Current drywell profiles were stable and consistent with established baseline profiles.
Tank Description

Type: Single Shell

Constructed: 1944

In-service: $06 / 23 / 46$

75

Capacity: $530 \mathrm{~K}$ gallons

Bottom shape: Dish

Hanford Coordinates:

$38,197^{\prime}$ North

75,737' West

Ventilation: Passive

\section{Leak Detection System}

Surface Level:

FIC Rtser- R-08

Manual Tape Riser- None

LOW Riser(s)- R-02 
WHC-EP-0625

This page intentionally left blank. 


\section{Tank 241-U-103}

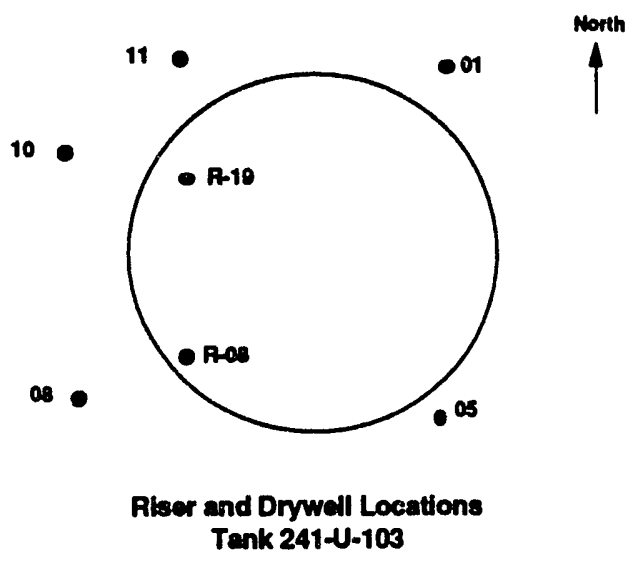

Number of External Drywells: 5

Number of Lateral Wells: None

\section{Tank Status}

Watch List: Hydrogen

Contents

Type: Non-Complexed Waste

Total Waste: $468 \mathrm{~K}$ gallons

Supernate Volume: 13K gallons

Drainable Interstitial Liquid: $176 \mathrm{~K}$ gallons

Isolation Status

Date Partially Interim Isolated: $12 / 15 / 82$

Surface Level/Leak Status

Integrity Category: Sound

FIC Surface Level: 166.65 Inches (12/28/92)

Last Photographed: 09/13/88

Photo Interpretation Comments:

Photographs from 10/01/81 and 09/28/84 show more surface liquid than previous photos with a large area of exposed salt cake floating on the surface. The FIC plummet is contacting liquid.

Temperature Status

Highest temperature during 1992: $91^{\circ} \mathrm{F}(11 / 05 / 92)$

Comments: Temperatures are stable.

Drywell Status

Comments: Current drywell profiles were stable and consistent with established baseline profiles. 
WHC-EP-0625

This page intentionally left blank. 
WHC-EP-0625

\section{Tank: 241-U-103}

\section{September 1988}

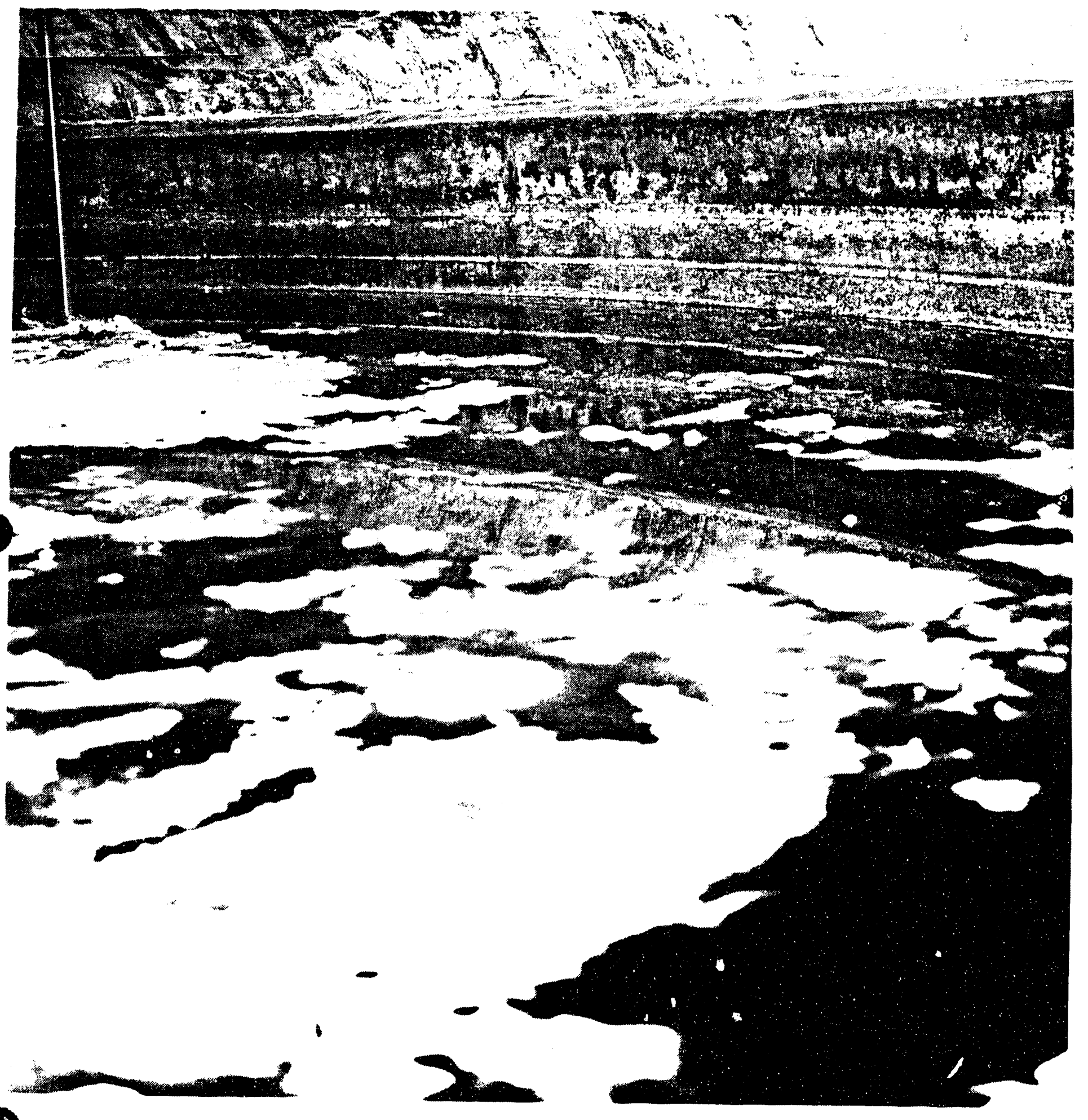

$88090946-13 \mathrm{CN}$

459 
WHC-EP-0625

This page intentionally left blank. 


\section{Tank 241-U-104}

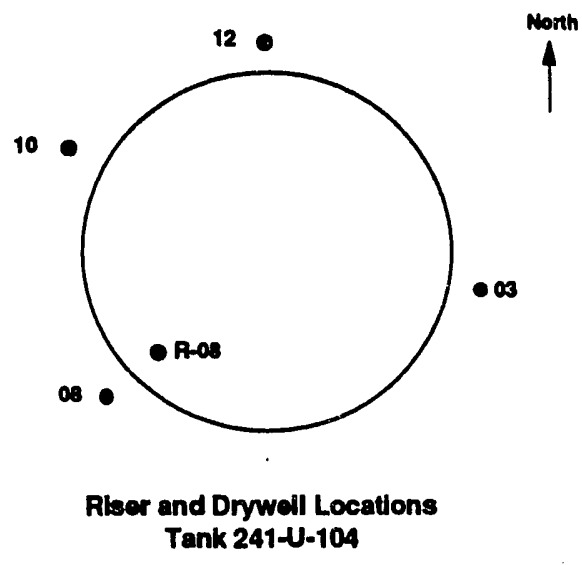

Number of External Drywells: 4

Number of Lateral Wells: None
Tank Description

Type: Single Shell

Constructed: 1944

In-service: $07 / 21 / 47$

Out of Service: 1960

Diameter: $7^{\circ}$

Usable Depth: 16'

Capacity: $530 \mathrm{~K}$ gallons

Bottom shape: Dish

Hanford Coordinates:

$38,097^{\prime}$ North

75,637' West

Ventilation: Passive

\section{Leak Detection System}

Surface Level:

FIC Riser- None

Manual Tape Riser- R-08

LOW Riser(s)- None

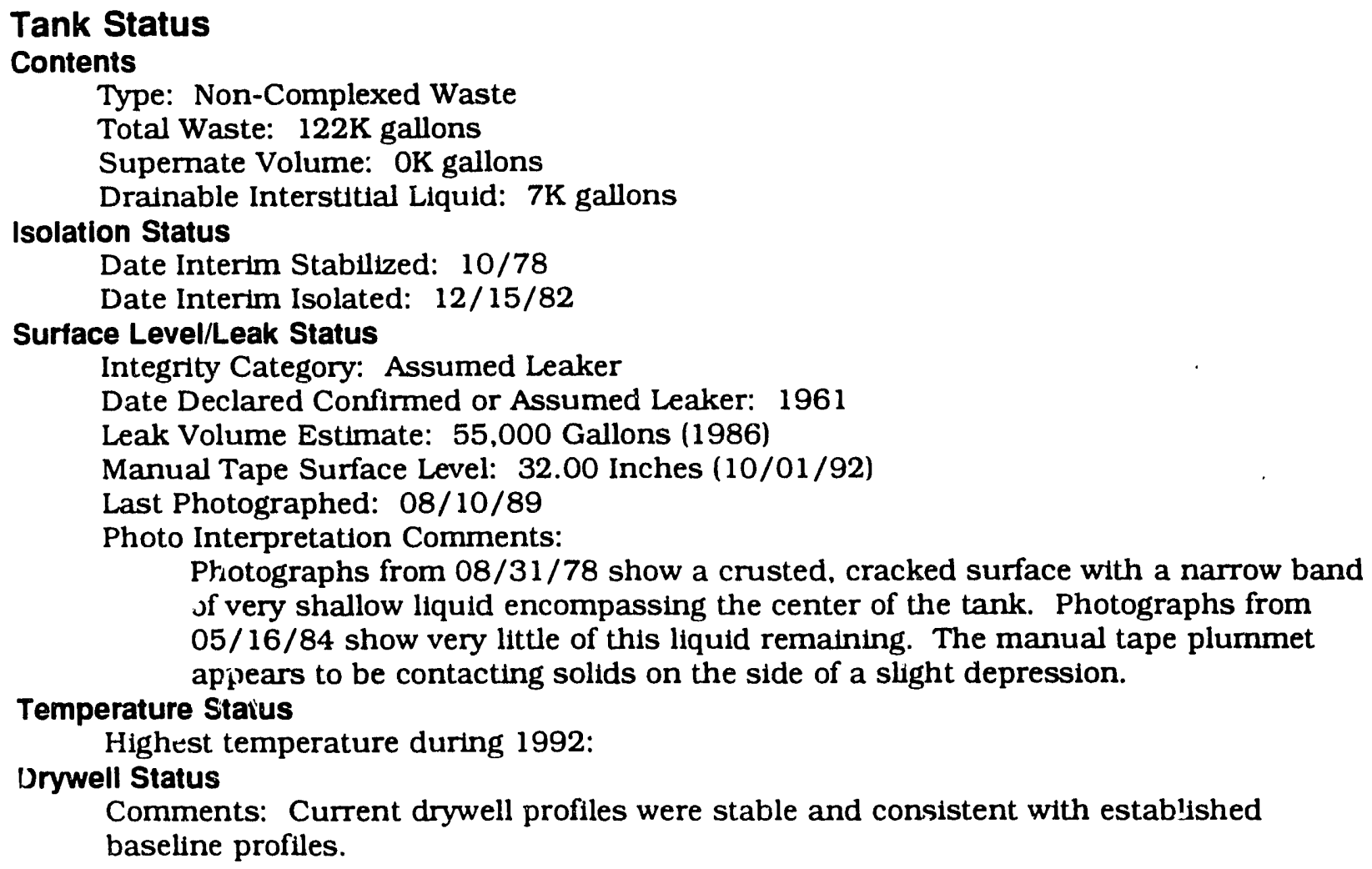

Comments: Current drywell profiles were stable and consistent with established baseline profiles. 


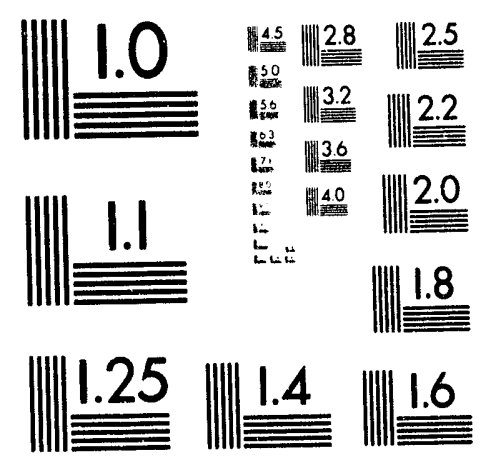



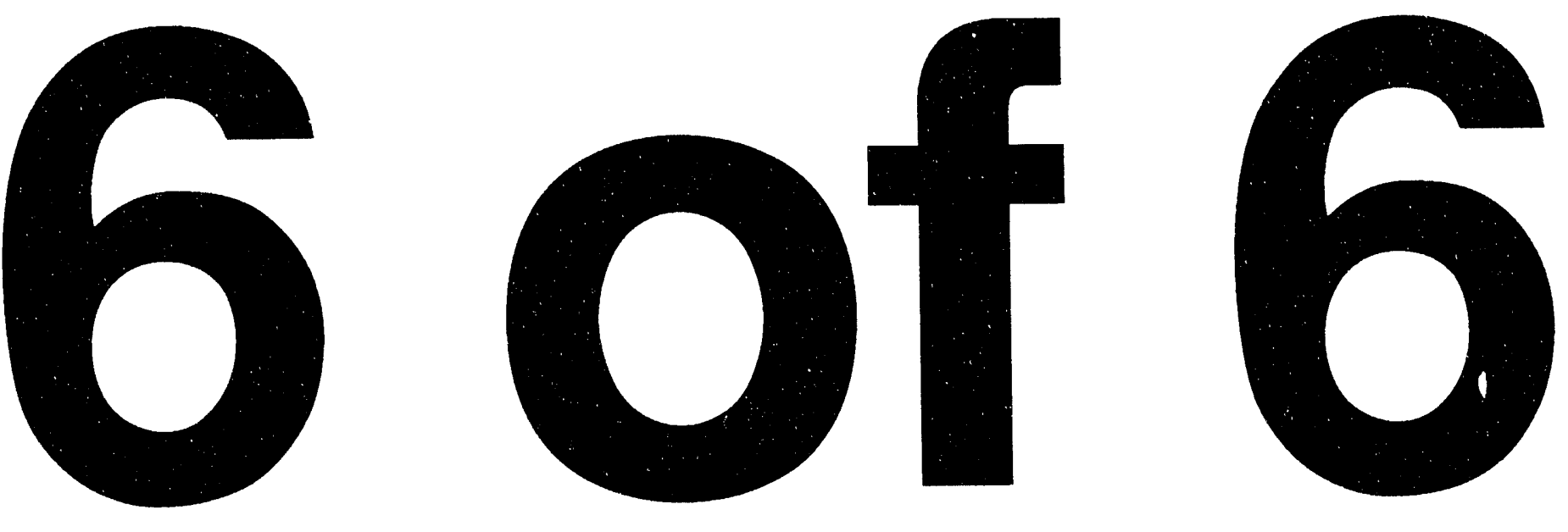
WHC-EP-0625

This page intentionally left blank. 


\section{Tank 241-U-105}

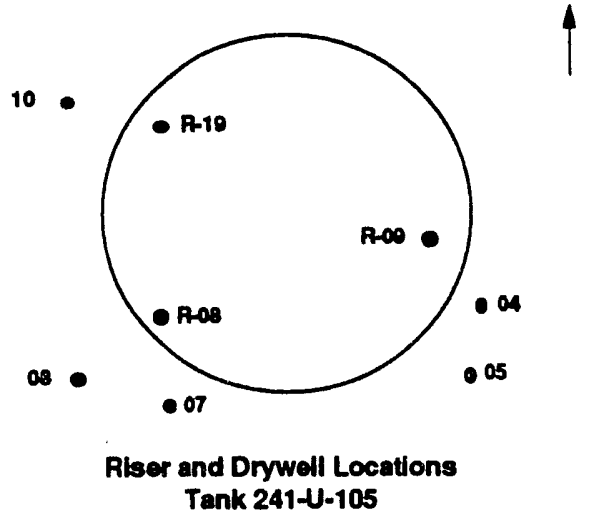

Number of External Drywells: 5 Number of Lateral Wells: None

\section{Tank Status}

\section{Watch List: Hydrogen}

\section{Contents}

Type: Non-Complexed Waste

Total Waste: $418 \mathrm{~K}$ gallons

Supernate Volume: $37 \mathrm{~K}$ gallons

Drainable Interstitial Liquid: $142 \mathrm{~K}$ galions

Isolation Status

Date Partially Interim Isolated: $12 / 15 / 82$

\section{Surface Level/Leak Status}

Integrity Category: Sound

FIC Surface Level: 149.75 Inches (12/28/92)

Last Photographed: 07/07/88

Photo Interpretation Comments:

Photographs show a surface of liquid and exposed salt cake with more surface liquid than previous photographs. The manual tape plummet is contacting liquid and the FIC plummet is contacting solids. The manual tape was removed on $08 / 09 / 85$.

\section{Temperature Status}

Highest temperature during 1992: $95^{\circ} \mathrm{F}(12 / 20 / 92)$

Comments: Temperatures are stable.

\section{Drywell Status}

Comments: Current drywell profles were stable and consistent with established baseline profiles.

Tank Description

Type: Single Shell

Constructed: 1944

In-service: $12 / 15 / 47$

Diameter: $75^{\prime}$

Usable Depth: 16'

Capacity: $530 \mathrm{~K}$ gallons

Bottom shape: Dish

Hanford Coordinates:

$38,097^{\prime}$ North

$75,737^{\circ}$ West

Ventllation: Passive

\section{Leak Detection System}

Surface Level:

FIC Riser- R-08

Manual Tape Riser- None

LOW Riser(s)- R-19 
WHC-EP-0625

This page intentionally left blank. 
Tank: 241-U-105

July 1988

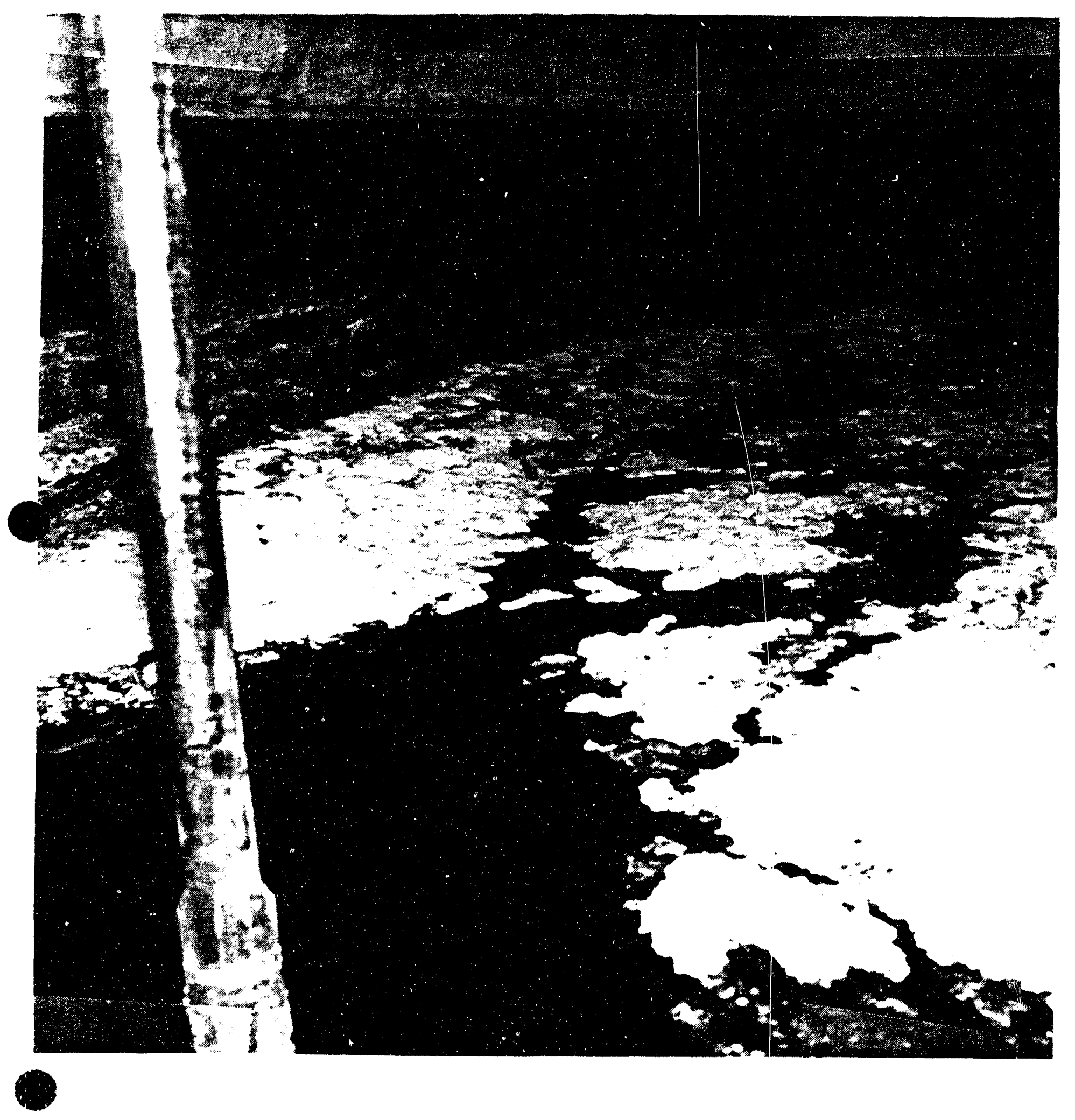

$8803818-6 \mathrm{CN}$ 


\section{WHC-EP-0625}

This page intentionally left blank. 


\section{Tank 241-U-106}

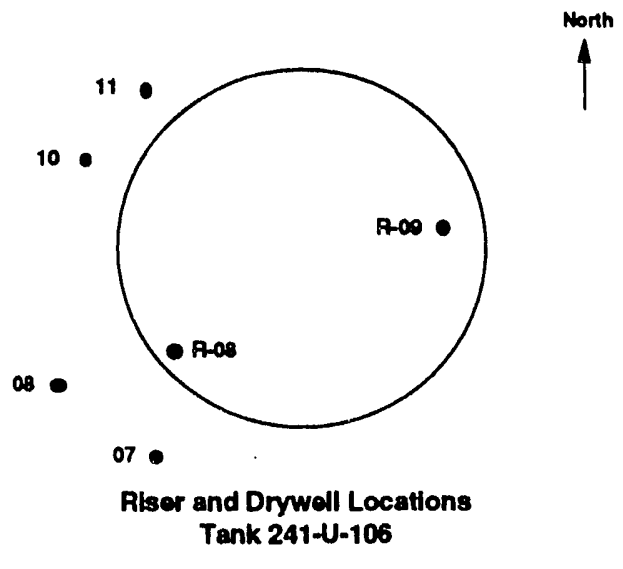

Number of External Drywells: 4 Number of Lateral Wells: None

\section{Tank Status}

\section{Watch List: Organic Salts}

Contents

Type: Non-Complexed Waste

Total Waste: $226 \mathrm{~K}$ gallons

Supernate Volume: $15 \mathrm{~K}$ gallons

Drainable Interstitial Liquid: $68 \mathrm{~K}$ gallons

Isolation Status

Date Partially Interim Isolated: $12 / 15 / 82$

\section{Surface Level/Leak Status}

Integrity Category: Sound

FIC Surface Level: 77.70 Inches (12/28/92)

Last Photographed: 07/07/88

Photo Interpretation Comments:

Photographs reveal a liquid surface that is free of solids and has a thin film visible in some areas. The FIC plummet is not visible because of haze, but would be contacting liquid.

\section{Temperature Status}

Highest temperature during 1992: $86^{\circ} \mathrm{F}(03 / 07 / 92)$

Comments: Temperatures are stable.

\section{Drywell Status}

Comments: Current drywell profiles were stable and consistent with established baseline profules.

\author{
Tank Description \\ Type: Single Shell \\ Constructed: 1944 \\ In-service: $05 / 28 / 48$ \\ Diameter: $7^{\circ}$ \\ Usable Depth: 16' \\ Capacity: $530 \mathrm{~K}$ gallons \\ Bottom shape: Dish \\ Hanford Coordinates: \\ 38,097' North \\ 75,837' West \\ Ventilation: Passive

\section{Leak Detection System} \\ Surface Level: \\ FIC Riser- R-08 \\ Manual Tape Riser- None \\ LOW Riser(s)- R-09
}


WHC-EP-0625

This page intentionally left blank. 
Tank 24106

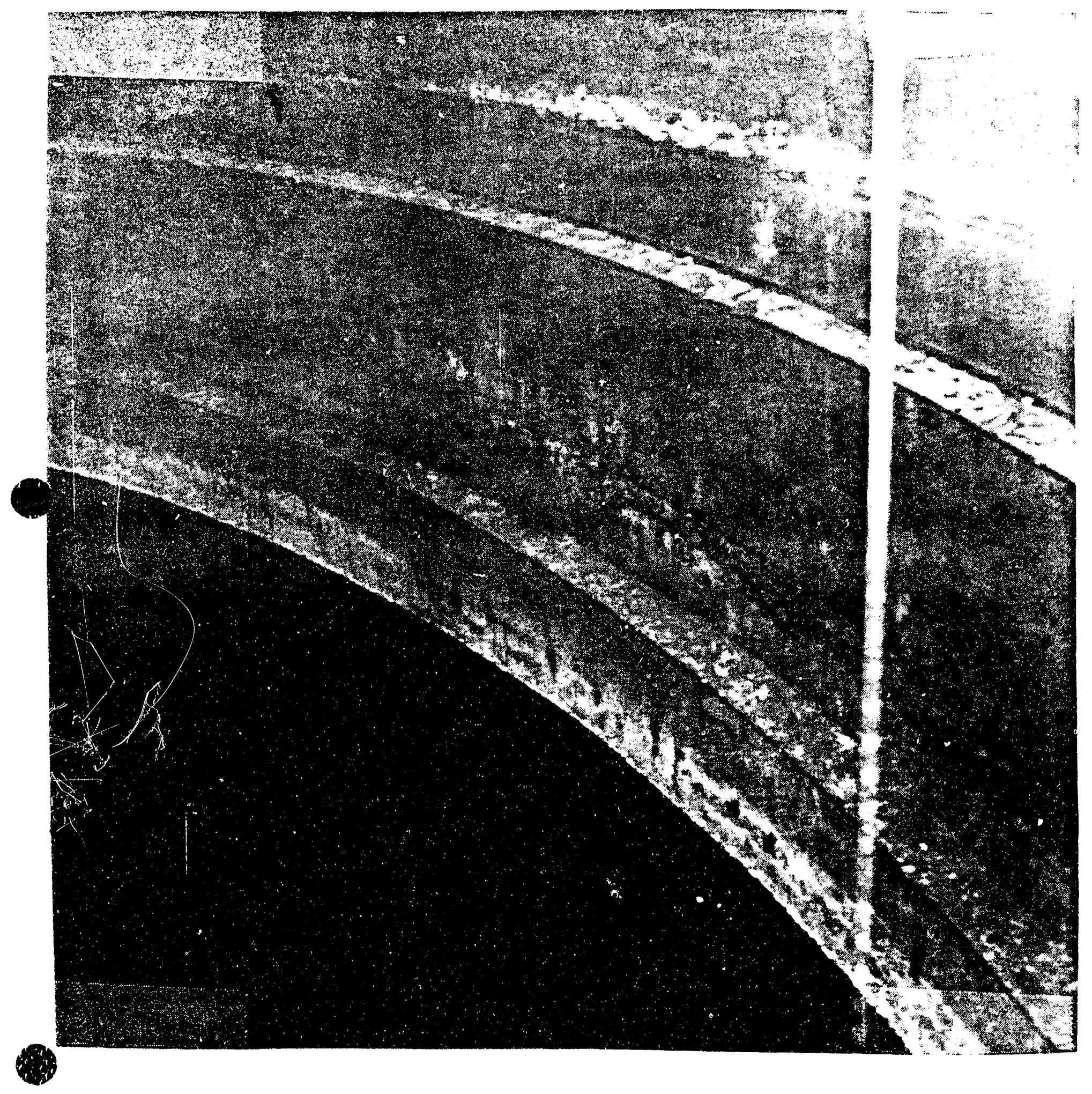


WHC-EP-0625

This page intentionally left blank. 


\section{Tank 241-U-107}

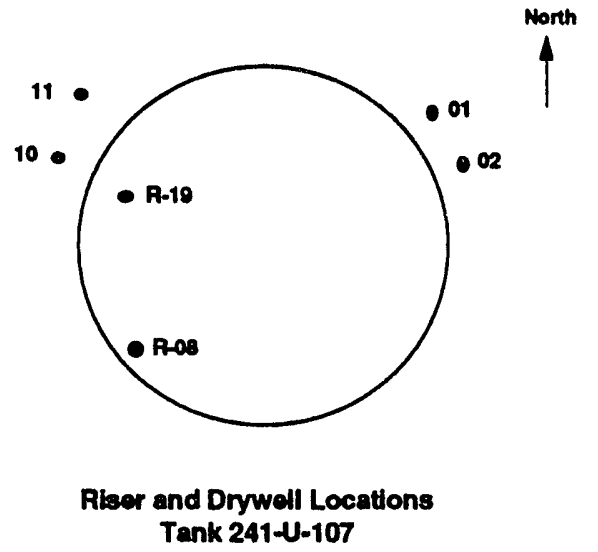

Number of External Drywells: 4

Number of Lateral Wells: None

\section{Tank Status}

\section{Watch List: Organic Salts}

Contents

Type: Double Shell Slurry Feed

Total Waste: $406 \mathrm{~K}$ gallons

Supernate Volume: $31 \mathrm{~K}$ gallons

Drainable Interstitial Liquid: $147 \mathrm{~K}$ gallons

Isolation Status

Date Partially Interim Isolated: 12/15/82

\section{Surface Level/Leak Status}

Integrity Category: Sound

FIC Surface Level: 144.10 Inches (12/28/92)

Last Photographed: 10/27/88

Photo Interpretation Comments:

Photographs reveal a liquid surface with patches of floating scum-like material over much of the surface area. The FIC plummet is contacting a clear liquid surface.

Temperature Status

Highest temperature during 1992: $84^{\circ} \mathrm{F}(11 / 05 / 92)$

Comments: Temperatures are stable.

Drywell Status

Comments: Current drywell profiles were stable and consistent with established baseline profiles.

\author{
Tank Description \\ Type: Single Shell \\ Constructed: 1944 \\ In-service: 09/27/48 \\ Diameter: $75^{\circ}$ \\ Usable Depth: 16' \\ Capacity: 530K gallons \\ Bottom shape: Dish \\ Hanford Coordinates: \\ 37,997' North \\ 75,637' West \\ Ventilation: Passive
}

\section{Leak Detection System}

Surface Level:

FIC Riser- R-08

Manual Tape Riser- None

LOW Riser(s)- R-19 
WHC-EP-0625

This page intentionally left blank. 
WHC-EP-0625

\section{Tank: 241-U-107}

July 1988

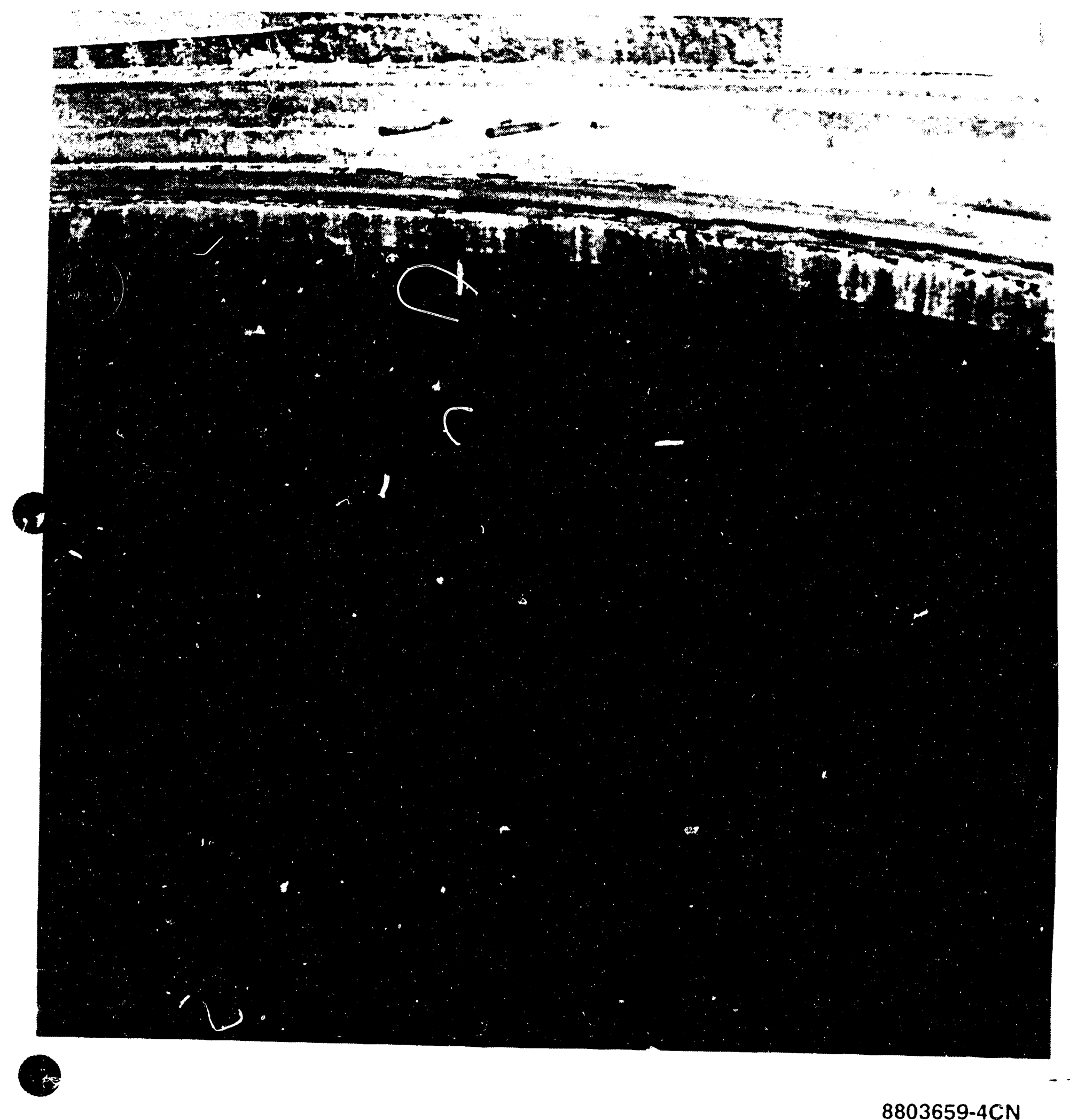


WHC-EP-0625

This page intentionally left blank. 


\section{Tank 241-U-108}

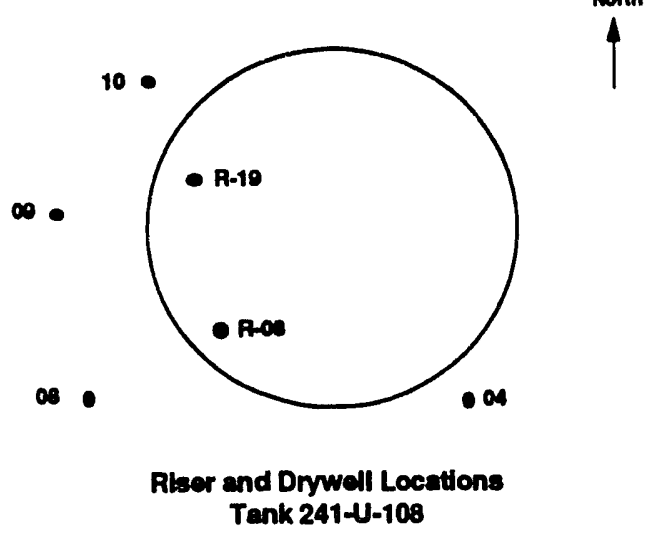

Number of External Drywells: 4

Number of Lateral Wells: None

\section{Tank Status}

\section{Watch List: Hydrogen}

Contents

Type: Non-Complexed Waste

Total Waste: $468 \mathrm{~K}$ gallons

Supernate Volume: $24 \mathrm{~K}$ gallons

Drainable Interstitial Liquid: $172 \mathrm{~K}$ gallons

Isolation Status

Date Partially Interim Isolated: $12 / 15 / 82$

\section{Surface Level/Leak Status}

Integrity Category: Sound

FIC Surface Level: Intrusion level set at 166.80 Inches $(12 / 28 / 92)$

Surface Level Comments: Intrusion Mode

Last Photographed: 09/12/84

Photo Interpretation Comments:

Photographs show what appears to be floating salt cake, with large areas of liquid visible. The intrusion mode FIC plummet appears to be suspended over solids, but is not readily visible.

\section{Temperature Status}

Highest temperature during 1992: $94{ }^{\circ} \mathrm{F}(07 / 17 / 92)$

Comments: Temperatures are stable.

Drywell Status

Comments: Current drywell profiles were stable and consistent with established baseline profiles. 
WHC-EP-0625

This page intentionally left blank. 
H:H:! !

Tank: 241-U-108

September 1984
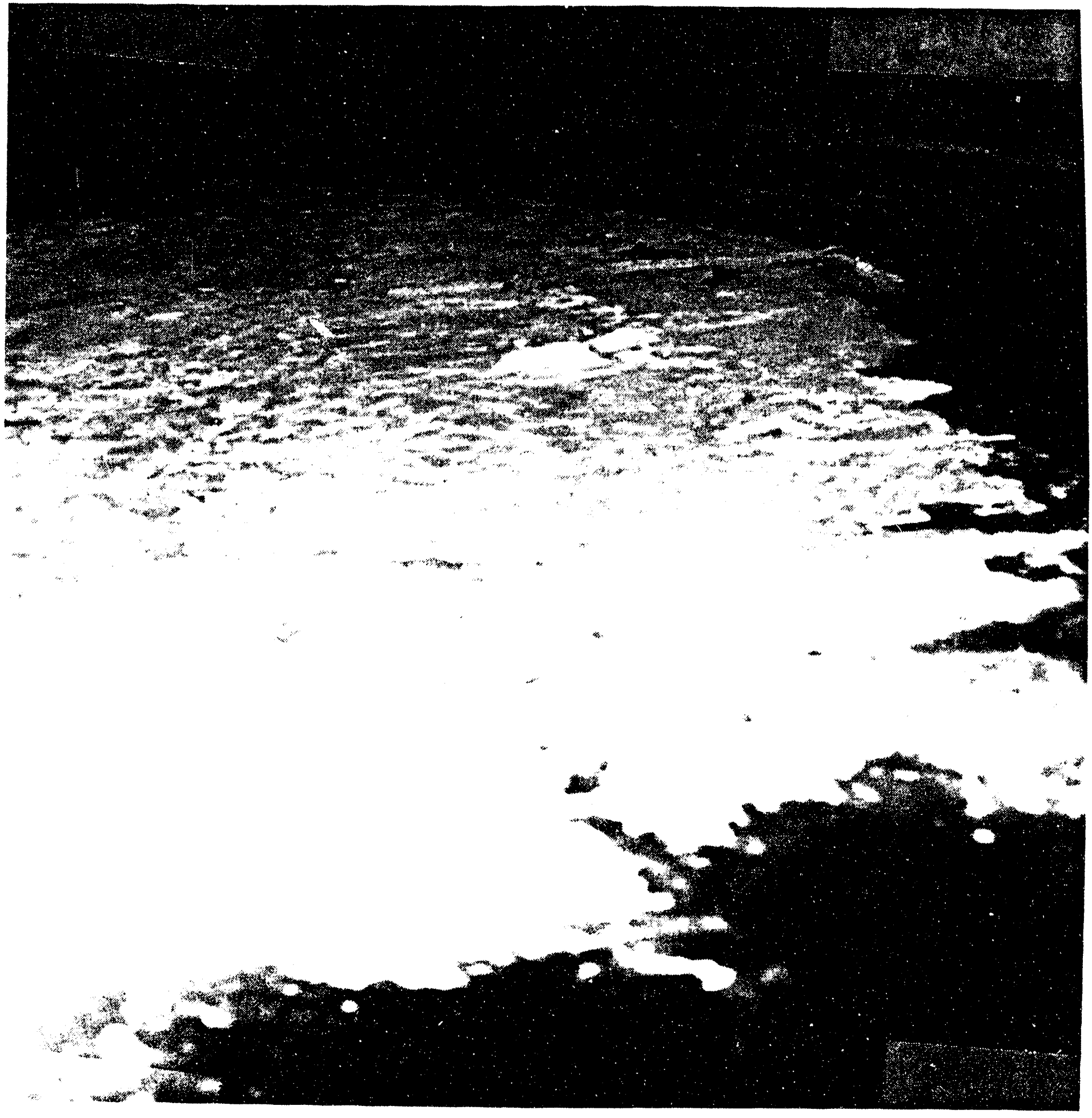

$9400 \cdot 3,0 \mathrm{~N}$ 
WHC-EP-0625

This page intentionally left blank. 


\section{Tank 241-U-109}

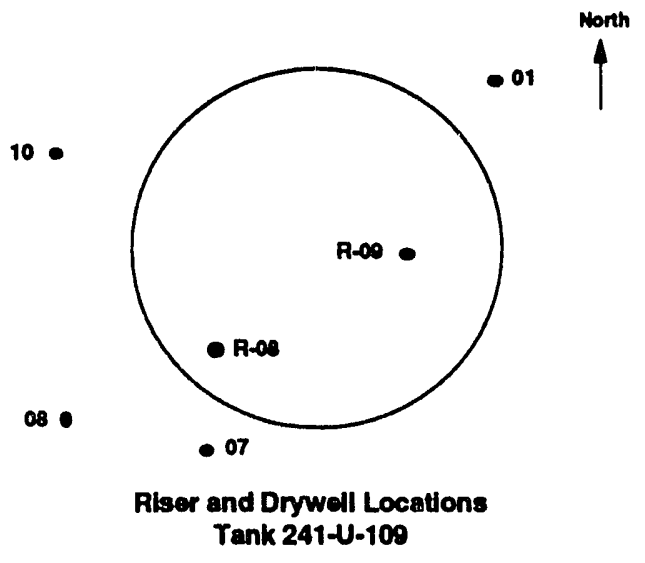

Number of External Drywells: 4

Number of Lateral Wells: None

\section{Tank Status}

Watch List: Hydrogen

Contents

Type: Non-Complexed Waste

Total Waste: $463 \mathrm{~K}$ gallons

Supernate Volume: 19K gallons

Drainable Interstitial Liquid: $163 \mathrm{~K}$ gallons

Isolation Status

Date Partially Interim Isolated: 12/15/82

\section{Surface Level/Leak Status}

Integrity Category: Sound

FIC Surface Level: 164.80 Inches $(12 / 28 / 92)$

Last Photographed: 07/07/88

Photo Interpretation Comments:

Photographs reveal a surface of exposed salt cake and large areas of clear liquid. The FIC plummet (not visible in current photos) is contacting liquid near the outer edge of the tank in $03 / 12 / 81$ photos.

\section{Temperature Status}

Highest temperature during 1992: $91{ }^{\circ} \mathrm{F}(03 / 01 / 92)$

Comments: Temperatures are stable.

Drywell Status

Comments: Current drywell proflles were stable and consistent with established baseline profiles.
Tank Description

Type: Single Shell

Constructed: 1944

In-service: $03 / 16 / 49$

Diameter: 75 '

Usable Depth: 16'

Bottom shape: Dish

Hanford Coordinates:

37,997" North

$75,837^{\circ}$ West

Ventilation: Passive

\section{Leak Detection System \\ Surface Level: \\ FIC Riser- R-08 \\ Manual Tape Riser- None \\ LOW Riser(s)- R-09}

Capacity: $530 \mathrm{~K}$ gallons 


\section{WHC-EP-0625}

This page intentionally left blank. 


\section{WHC-EP-0625}

\section{Tank: 241-U-109}

\section{July 1988}

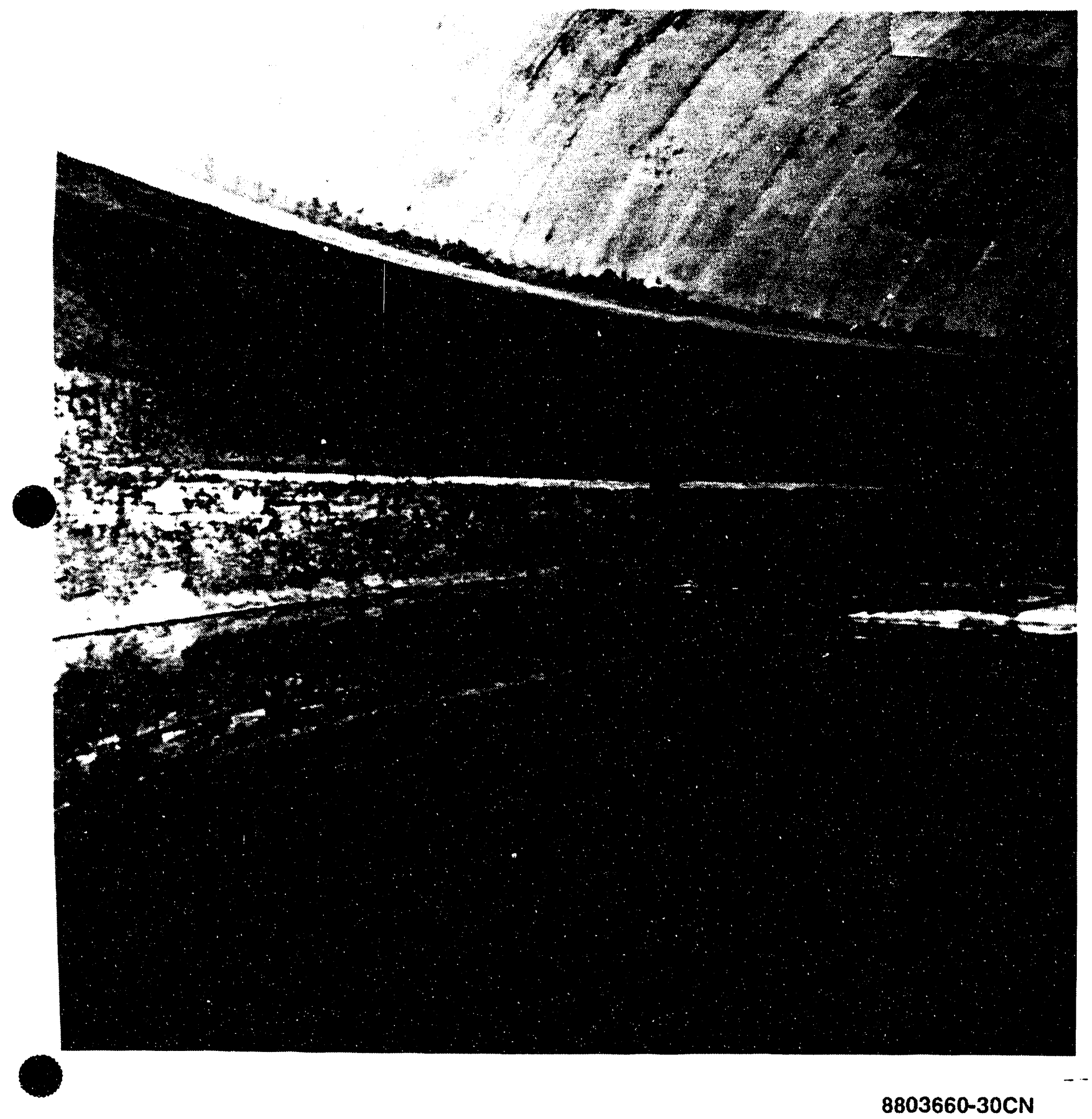




\section{WHC-EP-0625}

This page intentionally left blank. 


\section{Tank 241-U-110}

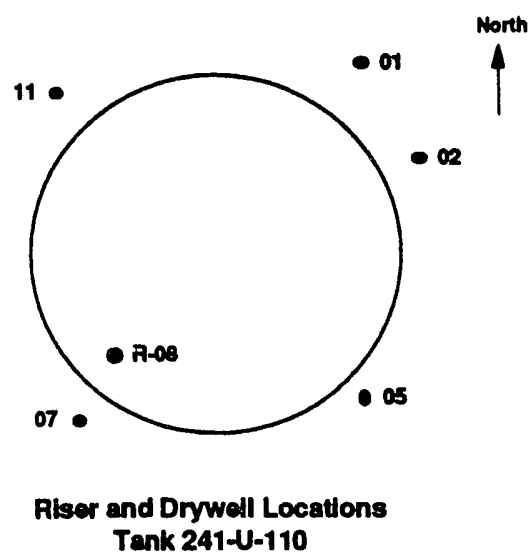

Number of External Drywells: 5

Number of Lateral Wells: None

\section{Tank Status}

Contents

Type: Non-Complexed Waste

Total Waste: $186 \mathrm{~K}$ gallons

Supernate Volume: OK gallons

Drainable Interstitial Liquid: $15 \mathrm{~K}$ gallons

Isolation Status

Date Interim Stabilized: $12 / 20 / 84$

Date Partially Interim Isolated: $12 / 15 / 82$

\section{Surface Level/Leak Status}

Integrity Category: Assumed Leaker

Date Declared Confirmed or Assumed Leaker: 1975

Leak Volume Estimate: 8,100 Gallons (1986)

FIC Surface Level: Intrusion level set at 61.80 Inches (12/28/92)

Surface Level Comments: Intrusion Mode

Last Photographed: 12/11/84

Photo Interpretation Comments:

Photographs from $07 / 12 / 79$ show a crusted surface with no visible liquid. The FIC plummet is contacting solids and a discarded tape is near the plummet. Photographs from $12 / 11 / 84$ that are clear show a crusted surface with no visible liquid.

Temperature Status

Highest temperature during 1992: $79.4^{\circ} \mathrm{F}(01 / 11 / 92)$

Comments: Temperatures are stable.

Drywell Status

Comments: Current drywell profiles were stable and consistent with established baseline profiles. 
WHC-EP-0625

This page intentionally left blank. 


\section{Tank 241-U-111}

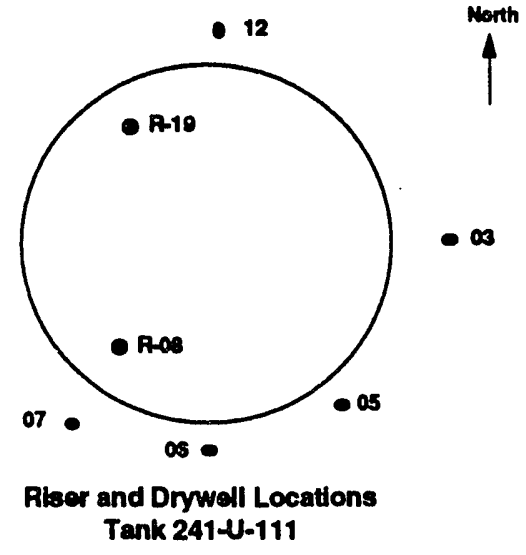

Number of External Drywells: 5

Number of Lateral Wells: None
Tank Description

Type: Single Shell

Constructed: 1944

In-service: $04 / 47$

Diameter: 75 '

Usable Depth: 16'

Capacity: $530 \mathrm{~K}$ gallons

Bottom shape: Dish

Hanford Coordinates:

37,897 North

75,737' West

Ventilation: Passive

\section{Leak Detection System}

Surface Level:

FIC. Riser- R-08

Manual Tape Riser- None

LOW Riser(s)- R-19

\section{Tank Status}

Contents

Type: Double Shell Slurry Feed

Total Waste: $329 \mathrm{~K}$ gallons

Supernate Volume: OK gallons

Drainable Interstitial Liquid: $122 \mathrm{~K}$ gallons

Isolation Status

Date Partially Interm Isolated: $12 / 15 / 82$

\section{Surface Level/Leak Status}

Integrity Category: Sound

FIC Surface Level: Intrusion level set at 118.00 Inches (12/28/92)

Surface Level Comments: Intrusion Mode

Last Photographed: 06/23/88

Photo Interpretation Comments:

Photographs show a dry, cracked, irregular surface that appears damp in some areas. The FIC plummet is directly over a large crack and measurement anomalies are to be expected.

Temperature Status

Highest temperature during 1992: $81^{\circ} \mathrm{F}(01 / 04 / 92)$

Comments: Temperatures are stable.

\section{Drywell Status}

Comments: Current drywell profiles were stable and consistent with established baseline profiles. 


\section{WHC-EP-0625}

This page intentionally left blank. 


\section{Tank 241-U-112}

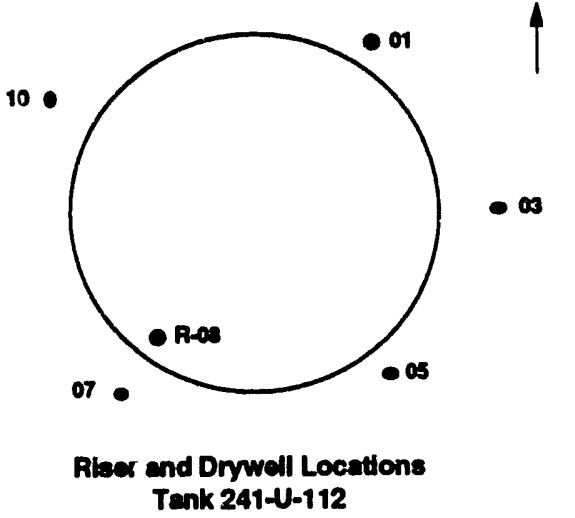

Number of External Drywells: 5 Number of Lateral Wells: None
Tank Description

Type: Single Shell

Constructed: 1944

In-service: $10 / 47$

Diameter: $7^{\circ}$

Usable Depth: 16'

Capacity: $530 \mathrm{~K}$ gallons

Bottom shape: Dish

Hanford Coordinates: $37,897^{\prime}$ North 75.837' West

Ventllation: Passive

\section{Leak Detection System}

Surface Level:

FIC Riser- None

Manual Tape Riser- R-08

LOW Riser(s)- None

\section{Tank Status}

Contents

Type: Non-Complexedi Waste

Total Wasie: $49 \mathrm{~K}$ gallors

Supernate Volume: $4 \mathrm{~K}$ gallons

Drainable Interstitial Liquid: OK gallons

Isolation Status

Date Interm Stabilized: 09/79

Date Interim Isolated: $12 / 15 / 82$

Surface Level/Leak Status

Integrity Categor r: Assumed Leaker

Daie Declared Confirmed or Assumed Leaker: 1980

Leak Volume Estimate: 8,500 Gallons (1986)

ManuaI Tape Surface Level: 12.50 Inches (10/01/92)

Last Photographed: 08/03/89

Photo Interpretation Comments:

Photographs from 07/31/81 reveal a shallow liquid surface and a sloping band of exposed solids around the perimeter of the tank. The manual tape plummet is contacting solids several feet from the liquid and a discarded tape is near the plummet. Photographs from $02 / 03 / 84$ show very little change from photographs taken on $07 / 31 / 81$.

\section{Temperature Status}

Highest temperature during 1992: $66.1^{\circ} \mathrm{F} \quad(01 / 04 / 92)$

Comments: Temperatures are stable.

\section{Drywell Status}

Comments: Current drywell profiles were stable and consistent with established baseline profilies. 
WHC-EP-0625

This page intentionally left blank. 


\section{WHC-EP-0625}

\section{GLOSSARY}

Active Tank. On all terms, a tank being used, or planned for use, for the storage of liquid in excess of a minimum supernatant liquid heel in connection with production and/or waste processing.

Aging Waste. High level, first-cycle solvent extraction waste from the PUREX plant neutralized cladding removal waste (NCAW).

Air Lift Circulator. Mildly agitates contents of waste tank by releasing compressed air into the waste (near the bottom) and allowing it to buoy up wastes in a pipe extending vertically through most of the waste. Its operating principle is identical to aquarium pumps that use compressed air to draw water up through a tube from an under-gravel filter. Circulation reduces the potential for solids settling in the waste tank.

Annulus. The space between the inner and outer shells on DSTs. Drain channels in the insulating and/or supporting concrete carry any leakage to the annulus space where conductivity probes are installed. Alarms from the annunciators are received by computer automated surveillance system (CASS). Continuous air monitoring (CAM) alarms are also located in the annulus. The annulus conductivity probes and radiation detectors are the primary means of leak detection for all DSTs.

Assumed Leaker. The integrity classification of a waste storage tank for which surveillance data indicate a loss of liquid attributed to a breach of tank integrity.

Automatic FIC. An automatic waste surface level measurement device manufactured by the Food Instrument Company (FIC). The instrument consists of a conductivity electrode (plummet) connected to a calibrated steel tape, a steel tape reel housing, and a controller that automatically raises and lowers the plummet to obtain a waste surface level reading. The controller can provide a digital display of the data and also transmit the reading to the CASS. Some tanks have gauges connected to CASS and others are read manually.

Bismuth Phosphate Process. First precipitation process used at the Hanford Site for separation of plutonium from the irradiated uranium fuels. This process was replaced by REDOX and PUREX processes to gain the advantages of separation and recovery of the uranium and plutonium fission products.

Boiling Waste. Waste containing sufficient radioactive decay heat to self-boil.

Bottom Referenced Tank. Either a dished bottom tank or a flat bottom tank where the zero point for liquid-level gages is the lowest elevation in the tank.

Bumping. A violent emission of vapor from a boiling liquid, contributed to by superheating. 
Burial Ground (Garden). A land area specifically designated to receive packaged contaminated wastes and equipment for burial.

Catch Tank. Small-capacity single-wall tank, primarily associated with diversion boxes and diverter stations. The tanks collect liquid from diversion boxes, diverter stations, catch stations, and other facilities.

Concentrated Complexant. Concentrated product from the evaporation of dilute complexed waste.

Concentrated Phosphate Waste. Waste originating from the decontamination of $100 \mathrm{~N}$ Area Reactor. Concentration of this waste produces concentrated phosphate waste.

Conductivity Probe. Measures surface level of conductive liquid (or waste) by detecting electrical conductivity between probe tip and liquid/waste surface as it is lowered into contact.

Crib. A subsurface drainage field used to dispose of low activity streams. The radionuclides are absorbed in the soil and the uncontaminated water percolates to ground water.

Crust. A hard surface layer that has formed in many waste tanks containing concentrated solutions.

Dilute Complexed Waste (DC). Characterized by a high content of organic carbon including organic complexants: ethylenediaminetetra-acetic acid (EDTA), citric acid, hydroxyethylenediaminetriacetic acid (HEDTA), and iminodiacetate (IDA) being the major complexants used. The main sources of dilute complexed waste in the DSTs system are salt well liquid inventory.

Dilute Noncomplexed Waste. Low activity liquid waste originating from $\mathrm{T}$ and $\mathrm{S}$ Plants, the 300 and 400 Areas, PUREX facility (decladding supernatant and miscellaneous wastes), 100 N Area (sulfate waste), B Plant, salt wells, and Plutonium Finishing Plant (PFP) (supernate).

Diversion Box. A below-grade concrete structure that contains jumpers and nozzles for routing liquid wastes to designated transfer or storage facilities.

Double-Shell Slurry (DSS). Waste that exceeds the sodium aluminate saturation boundary in the evaporator without exceeding receiver tank composition limits. For reporting purposes, DSS is considered a solid.

Double-Shell Slurry Feed. Waste concentrated just before reaching the sodium aluminate saturation boundary in the evaporator without exceeding receiver tank composition limits. This form is not as concentrated as DSS. 
Double-Shell Tank (DST). An underground waste storage tank with primary and secondary steel liners encased in a reinforced concrete shell.

Drainable Interstitial Liquid. Liquid that is not held in place by capillary forces, and will therefore migrate or move by gravity. Drainable liquid remaining minus supernate.

Drainable interstitial liquid is calculated based on the salt cake and sludge volumes, using average porosity values or actual data for each tank, when available.

Drainable Liquid Remaining. Supernate plus drainable interstitial.

Drywell. Vertical boreholes with 6-inch (internal diameter) carbon steel casings positioned radially around SSTs. Periodic monitoring is done by gamma radiation or neutron sensors to obtain scan profiles of radiation or moisture in the soil as a function of well depth, which could be indicative of tank leakage. These wells range between 50 and 250 feet in depth, and are monitored between the range of 50 to 150 feet. The wells are sealed when not in use. They are called drywells because they do not penetrate to the water table and are therefore usually "dry."

Drywell (in tank). A sealed casing within a tank that is attached to a riser and used for access of a gamma or neutron detector, or an acoustical probe to determine the level of interstitial liquid.

Ferrocyanide. A compound of iron and cyanide commonly expressed as FeCN. The actual formula for the ferrocyanide anion is $\left[\mathrm{Fe}(\mathrm{CN})_{6}\right]^{-4}$.

Food Instrument Corporation (FIC). The manufacturer of a surface level indicator found on many tanks. A plummet is lowered under machine control until electrical contact is established with the waste surface.

Hanford Coordinates. A set of offsets, in feet, from a reference point on the site. These are the units used to lay out these facilities. Conversion to latitude and longitude is possible.

High-Efficiency Particulate Air (filter). A filter designed to achieve 99.95 percent minimum efficiency in the containment of radioactive particulates greater than 0.3 micrometer in size.

In-Service Tank. The waste classification of a tank being used, or planned for use, for the storage of liquid (in excess of a minimum supernatant liquid heel) in conjunction with production and/or waste processing. All Hanford Site DSTs are in-service; none of the SSTs are in-service.

Interim Stabilized. A tank which contains less than 50,000 gallons of drainable interstitial liquid and less than 5,000 gallons of supernatant liquid. If the tank was jet pumped to achieve interim stabilization, then the jet pump flow must also have been at or below 0.05 gallons/minute before interim stabilization criteria is met. 
In-Tank Photography. In-tank photographs may be taken to aid in resolving in-tank measurement anomalies and determine tank integrity. Photographs help determine sludge and liquid levels by visual examination. Typically, an array of photos is taken to view the entire waste surface in a set of 12 or more images.

Interim Isolated. The administrative designation reflecting the completion of the physical effort required to minimize the addition of liquids into an inactive storage tank, process vault, sump, catch tank, or diversion box. Pipes in or out of a tank are typically blanked off, and risers and pits covered in a rigid foam (weather coated).

Interstitial Liquid. The liquid that fills the voids in the solids in the waste tank. This liquid is estimated to be about 50 percent of the solids volume: in salt cake, approximately 60 percent of the liquid is drainable and about 40 percent is held in place by capillary forces (nondrainable). In the sludge portion of the tank farm waste, this liquid is not considered pumpable or drainable but may contain pockets of liquid that cannot be estimated. Interstitial liquid may be evaporator feed or terminal liquid.

Intrusion. The unintended entry of any liquid into a waste storage tank.

Intrusion FIC. A mode of operating the FIC surface level monitoring equipment typically used when a waste surface is non-electrically conductive. The conductivity probe (plummet) is positioned a small distance above the waste surface. Should that gap be spanned by an intruding liquid, conductivity between the plummet and the waste surface would be established. This triggers an alarm in the CASS system. Note that the intrusion FIC level is not an actual measurement of the current waste surface.

Isolation. The act of sealing a tank against liquid intrusion from credible sources and confining the atmosphere in the tank. Filtered airways are not sealed. They balance the pressure to the atmosphere, and in some cases provide cooling airflow.

Jet Pump. A modified commercially available jet pump used as a salt well pump. A centrifugal pump recirculates a stream to serve as the motive fluid for the jet located at the bottom of the well.

Lance/Lancing. A long steel pipe, usually 2 to 3 inches in diameter. The top is bent at a 90-degree angle, and contains a check valve, gate valve, and nose connection. The bottom end of the lance is tapered to a 1/2-inch diameter. Water enters the top of the lance, which is forced out the bottom at high pressure. This creates a passage way which may be used for equipment installation (for example).

Lateral. Horizontal drywells positioned under single-shell waste storage tanks to detect radionuclides in the soil which indicate leakage. Lateral drywells are monitored by radiation detection probes. Laterals are 4-inch ID steel pipes located 8 to 10 feet below the tank's concrete base. There are three laterals per tank in A and SX Farms. There are no lateral drywells in any other farms. 
Leak Detection Pit. The collection point for leakage from AX Farm tanks and double-wall tanks. The pits are equipped with radiation and liquid-detection instruments.

Liquid Level (LL). The waste surface level within an underground storage tank.

Liquid Observation Well (LOW). Liquid observation wells are used for monitoring the interstitial liquid level (ILL) in single-shell waste storage tanks. The wells are constructed of fiberglass, or tefzel-reinforced epoxy-polyester resin. They extend to within 1 inch of the bottom of the tank steel liner. They are sealed at their bottom ends and have a nominal outside diameter of 3.5 inches. Three probes are used to monitor changes in the interstitial liquid level: acoustic, gamma, and neutron. They can indicate intrusions or leakage by increases or decreases in the interstitial liquid level. There are 58 liquid observation wells (57 are in operation) installed in SSTs that contain or are capable of containing greater than 50,000 gallons of drainable interstitial liquid, and in two DSTs only. The liquid observation wells installed in two DSTs (102-SY and 103-AW tanks only) are used for special surveillance purposes only.

Noncomplexed. General waste term applied to all Hanford Site liquids not identified as complexed.

Out-Of-Service. A tank which does not meet the definition of an in-service tank. All SSTs are out of service.

P-10 Pump. A turbine pump used in the first stage of removing liquids from a waste storage tank.

Partially Interim Isolated. The administrative designation reflecting the interim isolated completion of the physical effort required for interim isolation, except for isolation of risers and piping that is required for jet pumping or for other methods of stabilization.

Plutonium Finishing Plant (PFP) transuranic (TRU) Solids. Transuranic solids fraction from PFP operations.

Plummet. Weighted plumb bob at the lower end of the measuring tape on liquid-level gages.

Pond (Swamp). Ground area where uncontaminated or low-level waste water is discharged to seep into the ground.

Probe. An instrument package designed to be inserted in dry wells, tank risers, or other access ports to measure conductivity, radiation, moisture, or temperature.

Pumpable Liquid Remaining. Drainable liquid remaining less undrainable heel volume. 
PUREX Decladding. PUREX neutralized cladding removal waste (NCRW) is the solids portion of the PUREX plant neutralized cladding removal waste stream; the stream is received in tank farms as a slurry. Neutralized cladding removal waste solids are classified as transuranic (TRU) waste.

Riser. The upright pipe projection from a tank; any vertical pipe.

Sludge. Solids formed during sodium hydroxide additions to waste. Sludge usually was in the form of suspended solids when the waste was originally received in the tank from the waste generator. In-tank photographs may be used to estimate the volume.

Salt Cake. Results from crystallization and precipitation after concentration of liquid waste, usually in an evaporator. If salt cake is layered over sludge, it is only possible to measure total solids volume. In-tank photographs may be used to estimate the salt cake volume.

Salt Well. A screened well casing inserted into waste tank solids, extending to near the bottom of the tank. The well may contain a jet pump to remove interstitial liquid that drains into the well from surrounding solids.

Salt-Well Pump. A low-capacity pump used to remove interstitial liquid from salt wells.

Side Referenced Tank. A dished-bottom tank where the zero point for the liquid-level gages is at the elevation that the dished bottom begins.

Single-Shell Tank (SST). An underground waste storage tank with a single metal liner encased in a reinforced concrete shell.

Solids Update Source. Indicates the source or basis of the latest solids volume update.

Solids Volume Update. Indicates the latest update of any change in the solids volume.

Sound. The integrity classification of a waste storage tank for which surveillance data indicate no loss of liquid from a breach of integrity.

Supernate. The liquid above the solids in waste storage tanks.

Supernatant Liquid. Drainable liquid remaining minus drainable interstitial. Supernate is usually derived by subtracting the solids level measurement from the liquid level measurement.

Surface Levels. The surface level measurements in all waste storage tanks are monitored by manual or automatic conductivity probes, and recorded and transmitted or input to the CASS. 
Tank Farm. Area containing a number of storage tanks (e.g., the chemical tank farm, which stores chemicals used in a plant, or the underground waste tank farm, which stores radioactive waste).

Thermocouple (TC). A device used to measure temperature based on the Seebeck effect. An assembly with multiple thermocouples is called a tree. In DSTs there may be one or more thermocouple trees in risers in the primary tank. In addition, in DSTs only, there are thermocouple elements installed in the insulating concrete, the lower primary tank knuckle, the secondary tank concrete foundation, and in the outer structural concrete. These monitor temperature gradients within the concrete walls, bottom of the tank, and the domes.

In SSTs, there may be one or more thermocouple trees installed directly in a tank, although some SSTs do not have any trees installed. A single thermocouple may be installed in a riser, or lowered down an existing riser or liquid observation well. There are also four thermocouple laterals beneath tank 105-A in which temperature readings are taken in 34 thermocouples.

Total Waste. Solids volume plus supernatant liquid.

Watch List. A list of tanks that may pose an offsite release hazard from the following potential sources: trap and accumulate hydrogen gas, contain organic salts, contain ferrocyanide, or have a high-heat generation rate. This list is a result of a bill passed by Congress, Public Law 101-510, Section 3137, "Safety Measures for Waste Tanks at Hanford Nuclear Reservation." This law has become known at the Hanford Site as the Wyden Amendment (sponsored by Congressman Wyden from Oregon). The bill provided specific direction to the Hanford Site operating contractor for monitoring tanks with certain potential hazards. 
WHC-EP-0625

This page intentionally left blank. 


\section{REFERENCES}

Boyles, V. C., 1990, Single-Shell Tank Stabilization Record, WHC-SD-RE-TI-178, Rev. 1, Westinghouse Hanford Company, Richland, Washington.

This document provided the stabilization dates shown on the summary data sheets.

Fíailon, B. M., 1992, Tank Farm Surveillance and Waste Status Summary Report for September 1992, WHC-EP-0182-54, Westinghouse Hanford Company, Richland, Washington.

This document was the source for the following summary tank data sheet information: total waste, supernatant, drainable interstitial, contents, status, ventilation tank type, leak date, leak estimate, and Watch List reason.

Hanlon, B. M., 1993, Interview with E. I. Husa, Westinghouse Hanford Company, Richland, Washington, January 1993.

This interview concerned the interpretation of the temperature trends. The temperature readings were extracted from the Surveillance Analysis Computer System.

National Defense Authorization Act for Fiscal Year 1991, Public Law 101-510, "Safety Measures for Waste Tanks at Hanford Nuclear Reservation," Section 3137 (Wyden Amendment).

Rios, R. R., 1992 to 1993, Surface level logbooks, covering the daily, weekly, and quarterly surface-level measurements for the single- and double-shell waste storage tanks in the 200 East and West Areas of the Hanford Site, Westinghouse Hanford Company, Richland, Washington.

These logbooks were the source for all information pertaining to surface-level measurements, including manual tape and FIC surface-level readings.

Vermeulen, N. J., 1993, Interview with E. I. Husa, Westinghouse Hanford Company, Richland, Washington, January 1993.

This interview was the source for information on the drywells.

Welty, R. K., 1988, Waste Storage Tank Status and Leak Detection Criteria, "Waste Tank Data Commentaries," WHC-SD-WM-TI-356, Rev. 0, Westinghouse Hanford Company, Richland, Washington. 
This document was the source for the following information on the data sheets: date constructed, in-service date, out-of-service date, date interim stabilized, date partially isolated, date isolated, diameter, height, capacity, total waste, bottom shape, manual tape riser number, FIC riser number, and LOW riser number. 
WHC-EP-0625

\section{DISTRIBUTION}

\section{Number of Copies}

ONSITE

U.S. Department of Energy.

Richland Operations Office

Public Reading Room

A1-65

3

Pacific Northwest Laboratory,

Richland Washington

J. B. Colson (PNL)

K5-10

K. E. Noffsinger (PNL)

K5-20

Technical Files

P8-55

Westinghouse Hanford Company.

Richland. Washington

H. Babad

R2-78

M. V. Berrichoa

B3-30

J. G. Burke Jr.

B3-25

S. J. Dechter

R2-54

C. deFigh-Price

R2-31

M. F. Erhart

R1-51

J. C. Fulton

R2-31

S. D. Godfrey

R1-51

J. G. Hill

R2-12

E. I. Husa

R1-80

D. B. Smet

X5-52

R. R. Stickney

R1-49

D. A. Turner

R2-78

T. S. Vail

R1-43

M. P. Whiting

B3-25

C. M. Winkler

L4-75

D. D. Wodrich

H0-30

Tank Farm Information Center

R1-20

Central Files

L8-04

Information Release Administration (3)

R1-08

Publications Services

R1-08 

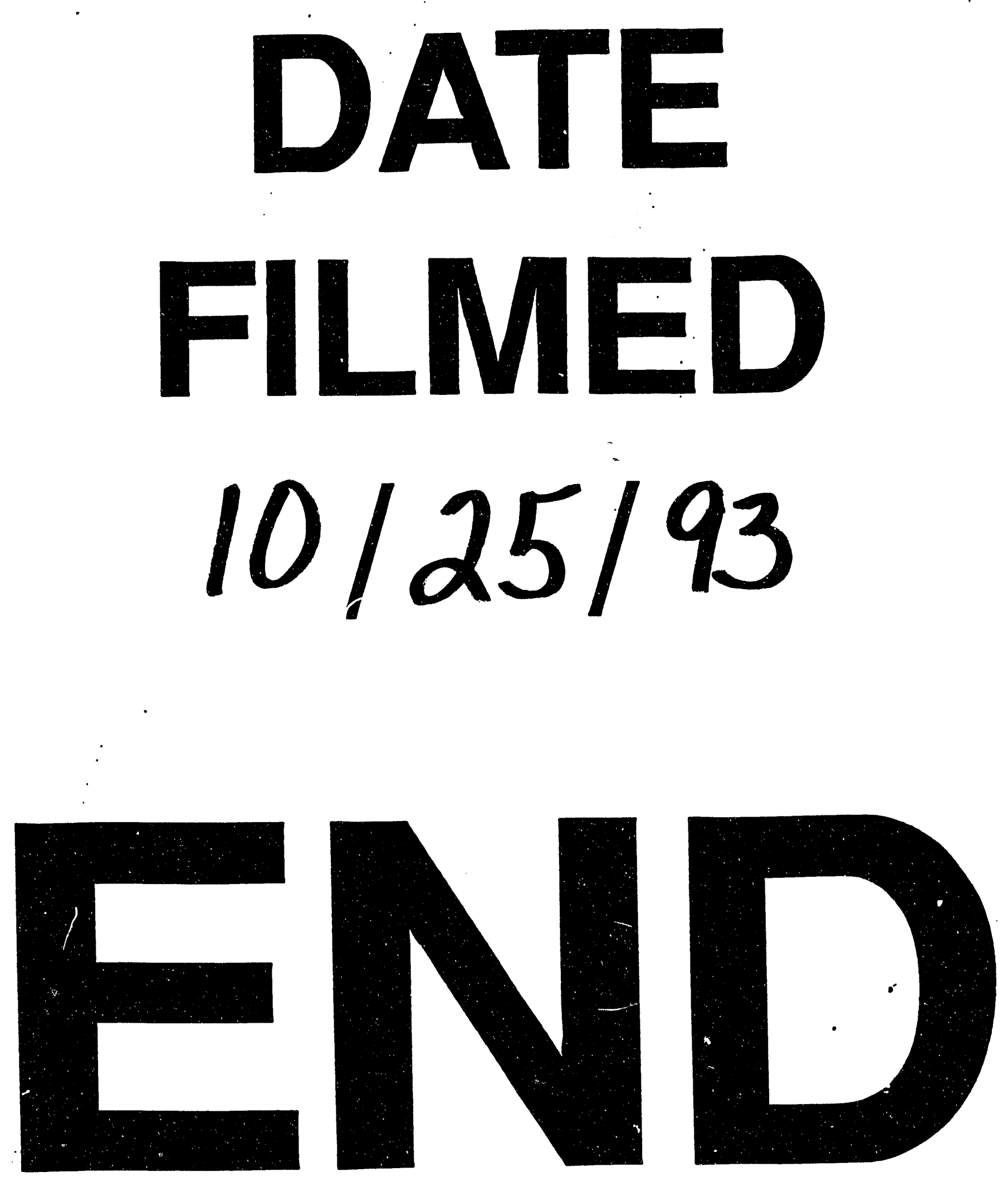